
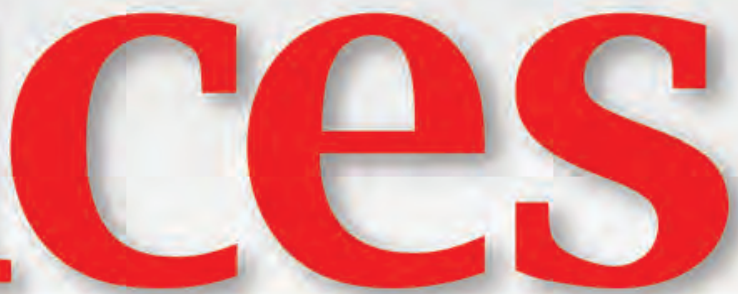

\title{
of the American Mathematical Society
}

November 2017

Volume 64, Number 10

Antibiotics Time Machines

Are Hard to Build

page 1136

Fall Western Sectional Sampler

page 1142

Backlog of Mathematics Research Journals

\section{page 1184}

Biennial Overview of AMS Honors

\section{page 1200}
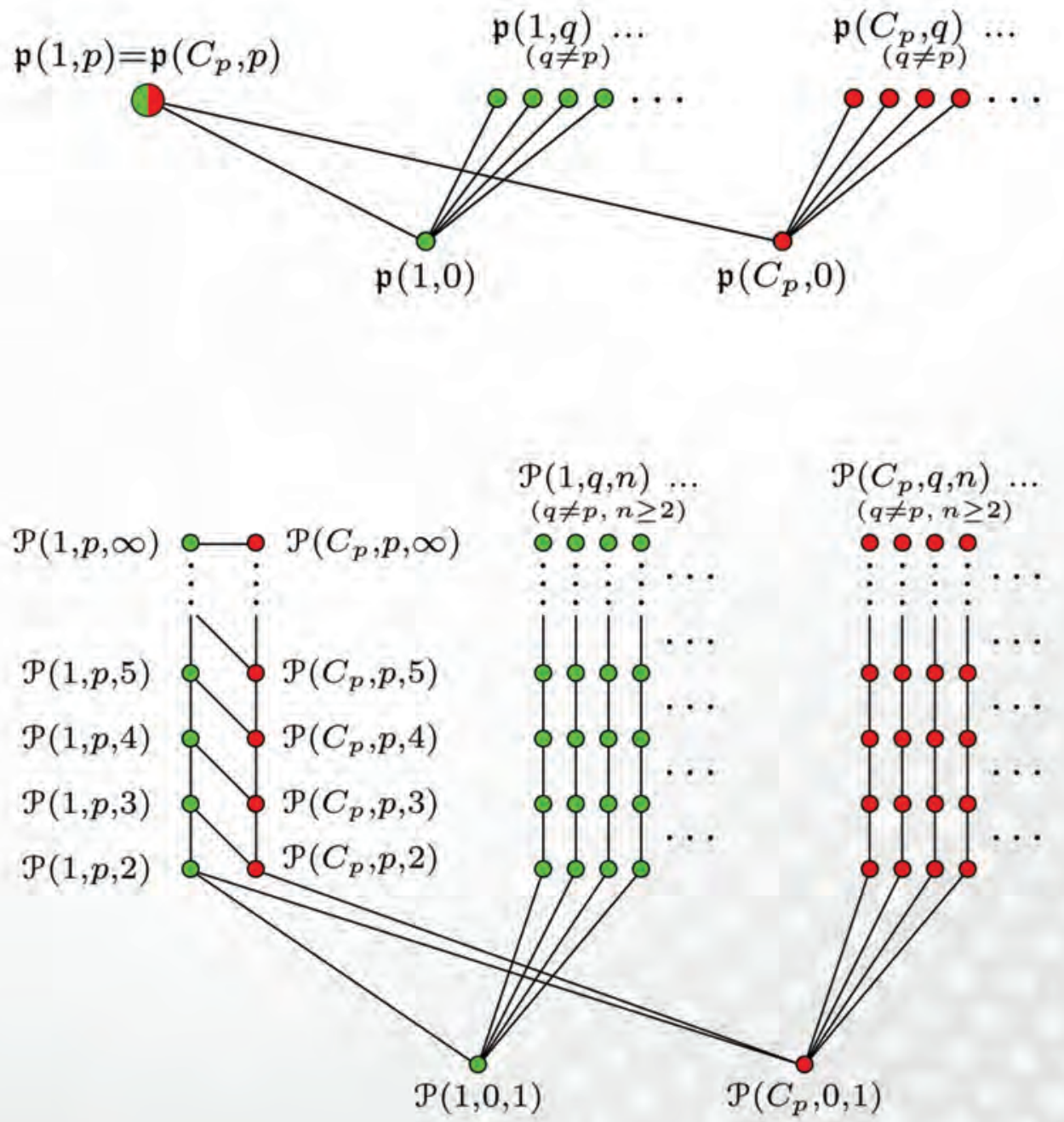


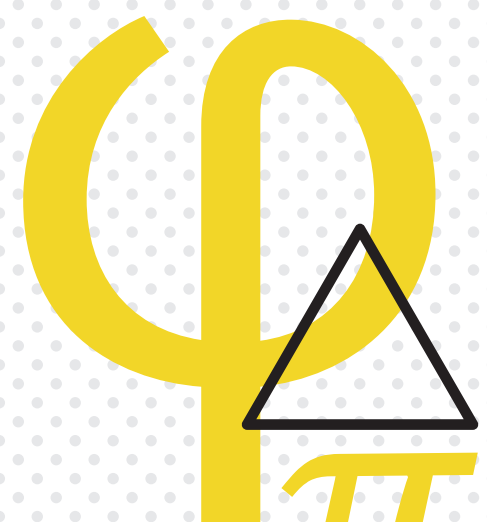

นก

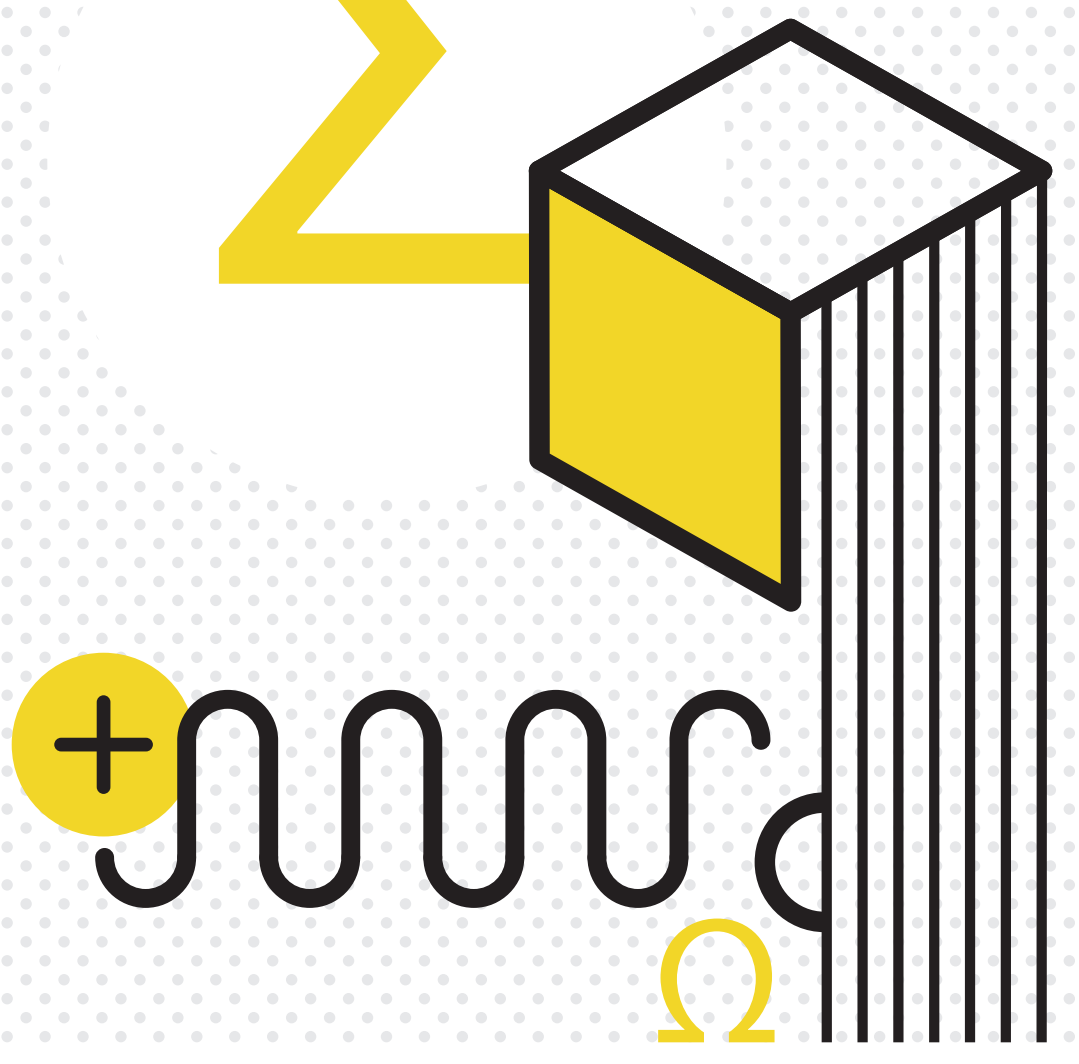

Homework Assignments

Nagwa offers free access to a comprehensive library of mathematics questions written by subject experts and tagged with topic keywords. Creating customized homework assignments for any of your classes couldn't be easier!

\section{Why Use Nagwa for Homework Assignments?}

$>25,000+$ unique mathematics questions

$>$ Millions of question variations

$>$ Free to schools, teachers, and students

$>$ Covers K-12 and early college mathematics

$>$ Eliminate time spent on grading

$>$ Individual student performance reports 


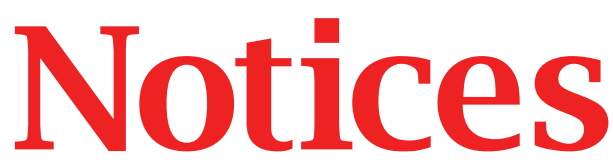

of the American Mathematical Society

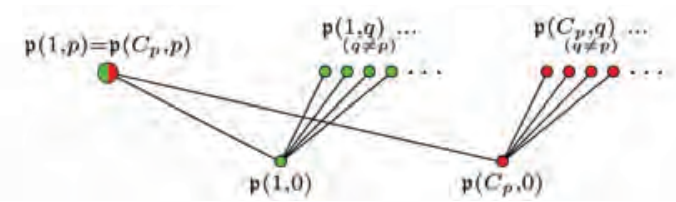

November 2017

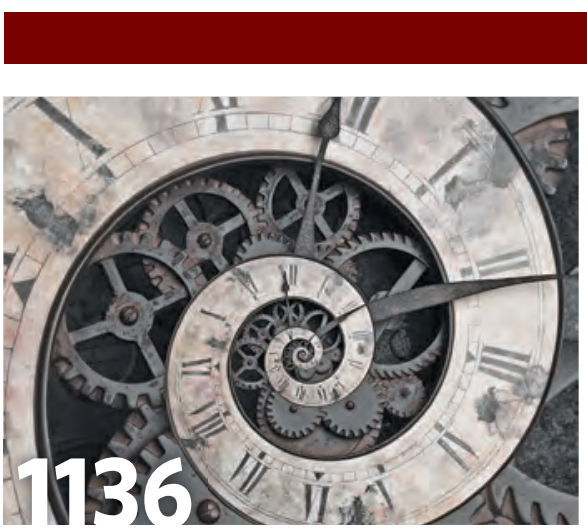

Antibiotics Time

Machines Are Hard to Build

by Ngoc M. Tran and Jed Yang

FEATURED

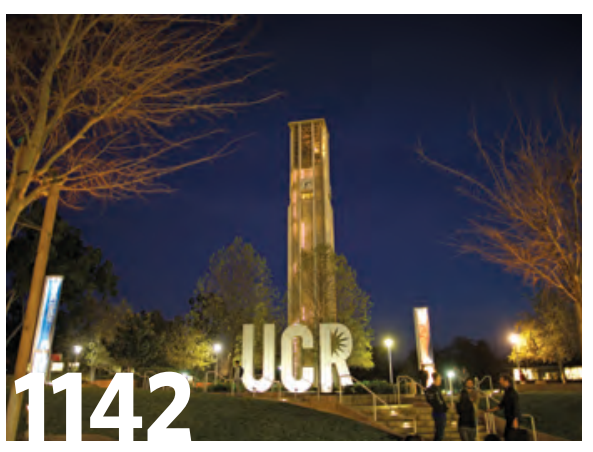

AMS Fall Western Sectional Sampler

An Invitation to Tensor-Triangular Geometry by Paul Balmer

Double Affine Hecke Algebras and Their Applications by Pavel Etingof

Combinatorics, Categorification, and Crystals by Monica Vazirani

November, the month of the American Thanksgiving, seems an appropriate time to be grateful for mathematics, for our community, and especially for you our Notices readers. In appreciation we offer a feature on the difficulty of undoing the problem of antibiotic-resistant microbes, a sampler from the AMS Fall Western Sectional, a deep glimpse into the concept of varifold, a preview of the AMS two-day short course on discrete differential geometry, an account of immigration in the history of the Princeton Institute for Advanced Study, the Graduate Student section, and advice on writing a teaching recommendation letter and on making mathematics accessible to people with hearing loss. - Frank Morgan, Editor-in-Chief

\section{ALSO IN THIS ISSUE}

1153 A Glimpse into Discrete Differential Geometry by Keenan Crane and Max Wardetzky

1160 Immigration, Freedom, and the History of the Institute for Advanced Study by Fadi Bardawil, Thomas Dodman, Ian Jauslin, Pascal Marichalar, Klaus Oschema, and Peter Redfield

1198 AMS Reciprocity Agreements

\section{FROM THE AMS SECRETARY}

11412018 AMS Elections: Call for Suggestions

1184 Backlog of Mathematics Research Journals

1200 Biennial Overview of AMS Honors

\section{COMMENTARY}

1133 Letters to the Editor

1178 Opinion: Writing a Teaching Letter

Chris Woodward

1180 Opinion: Accessible Mathematics for People with Hearing Loss at Colleges and Universities J. Tilak Ratnanather

1191 Book Review: The Slow Professor David Manderscheid

\section{GRADUATE STUDENT SECTION}

1170 Lillian Pierce Interview Laure Flapan

1173 WHAT IS... a Varifold? Editors

1174 AMS Graduate Student Blog 


\section{Notices}

of the American Mathematical Society

\section{JOINT MATHEMATICS MEETINGS}

1247 San Diego Meeting Registration Forms

\section{IN EVERY ISSUE}

\section{BookShelf}

1196 The Back Page

1208 Mathematics Opportunities

1211 Mathematics People

1217 Inside the AMS

1219 New Publications Offered by the AMS

1226 Mathematics Calendar

1228 Classified Advertising

1234 Meetings and Conferences of the AMS

\section{A Mathematical Gallery}

AVAILABLE IN EBOOK FORMAT

\section{Lisl Gaal}

Embark on a playful mathematical tour, aided by Lisl Gaal's illustrations of familiar scenes and whimsical triggers for the imagination. Along the way, find fruit stands arranged using polynomial multiplication, checkerboard tablecloths sewn with patterns of primes in a two-dimensional number system, and deceptive cats revealing that simple counting is not always so simple. This is a book to read and revisit, gaining new insights each time.

2017; 64 pages; Softcover; ISBN: 978-1-4704-41593; List US\$25; AMS members US\$20; Order code $\mathrm{MBK} / 111$

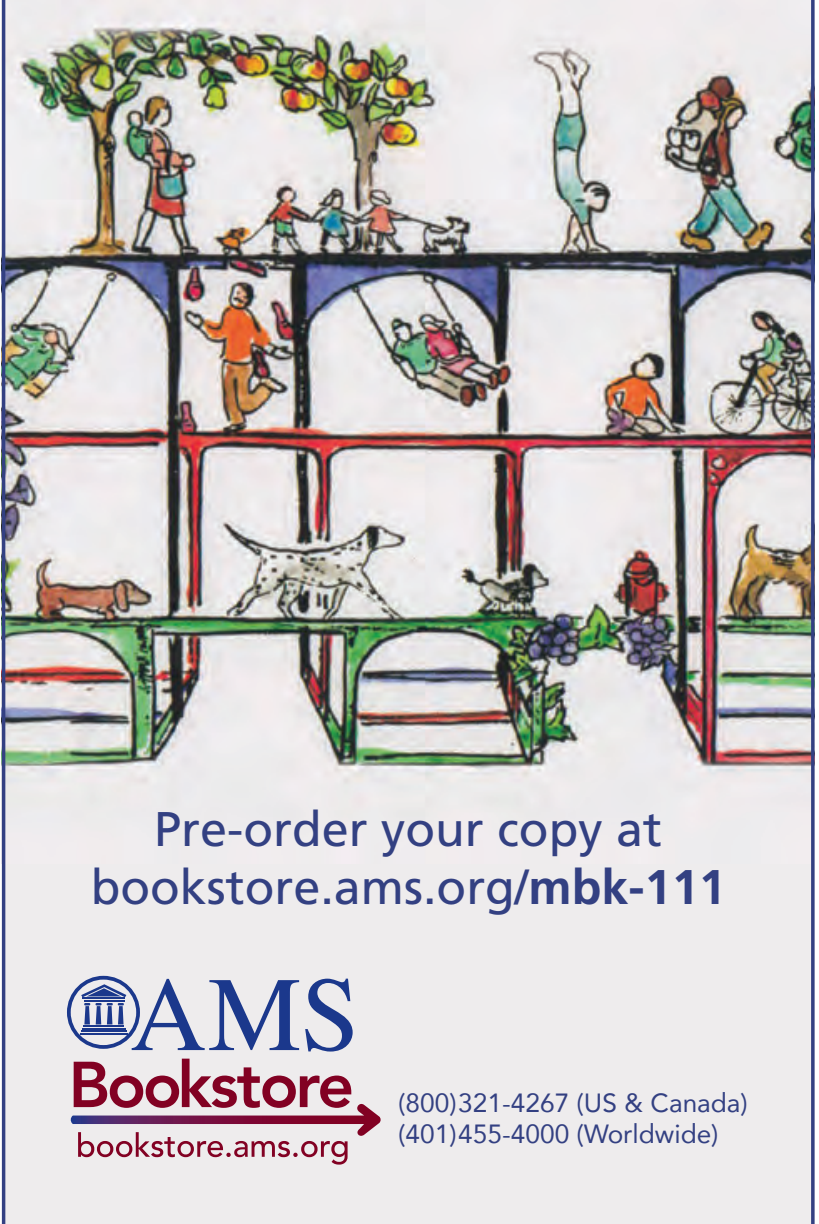




\section{EDITOR-IN-CHIEF \\ Frank Morgan}

\section{ASSOCIATE EDITORS}

Benjamin Braun

Alexander Diaz-Lopez, Thomas Garrity, Joel Hass,

Stephen Kennedy, Florian Luca, Steven J. Miller,

Harriet Pollatsek, Carla Savage (ex officio),

Cesar E. Silva, Christina Sormani, Daniel J. Velleman

CONSULTANTS

John Baez, Hélène Barcelo, Ricardo Cortez, Jerry Folland, Tara Holm, Kenneth A. Ribet

ASSISTANT to the EDITOR-IN-CHIEF Sophia D. Merow

\section{SENIOR WRITER/DEPUTY EDITOR \\ Allyn Jackson \\ MANAGING EDITOR Rachel L. Rossi \\ ADVERTISING COORDINATOR \\ Anne Newcomb \\ REPRINT PERMISSIONS \\ Erin M. Buck \\ CONTRIBUTING WRITER \\ Elaine Kehoe \\ COMPOSITION, DESIGN and EDITING \\ John Brady, Anna Hattoy, Lori Nero, Karen Ouellette, \\ Becky Rivard, Courtney Rose, Deborah Smith, Mike Southern, Peter Sykes}

Supported by the AMS membership, most of this publication, including the opportunity to post comments, is freely available electronically through the AMS website, the Society's resource for delivering electronic products and services. Use the URL www.ams.org/notices/to access the Notices on the website. The online version of the Notices is the version of record, so it may occasionally differ slightly from the print version.

The print version is a privilege of Membership. Graduate students at member institutions can opt to receive the print magazine by updating their individual member profiles at https://www.ams. org/cml/update-ams For questions regarding updating your profile, please call 800-321-4267.

For back issues see www.ams.org/backvols. Note: Single issues of the Notices are not available after one calendar year.

\section{CONTACTING THE NOTICES}

\section{SUBSCRIPTION INFORMATION}

Subscription prices for Volume 64 (2017) are US\$637 list; US\$509.60 institutional member; US\$382.20 individual member; US $\$ 573.30$ corporate member. (The subscription price for members is included in the annual dues.) A late charge of $10 \%$ of the subscription price will be imposed upon orders received from non-members after January 1 of the subscription year. Add for postage: Surface delivery outside the United States and IndiaUS\$27; in India-US\$40; expedited delivery to destinations in North America-US\$35; elsewhere-US\$120. Subscriptions and orders for AMS publications should be addressed to the American Mathematical Society, P.O. Box 845904, Boston, MA 02284-5904 USA. All orders must be prepaid.

\section{ADVERTISING}

Notices publishes situations wanted and classified advertising, and display advertising for publishers and academic or scientific organizations. Advertising requests, materials, and/or questions should be sent to:

\section{classads@ams.org (classified ads) notices-ads@ams.org (display ads)}

\section{PERMISSIONS}

All requests to reprint Notices articles should be sent to: reprint-permission@ams.org

\section{SUBMISSIONS}

The editor-in-chief should be contacted about articles for consideration after potential authors have reviewed the "For Authors" page at www.ams.org/publications/journals/notices/ noticesauthors.

The managing editor should be contacted for additions to our news sections and for any questions or corrections. Contact the managing editor at: notices@ams.org

\section{Letters to the editor should be sent to: notices-letters@ams.org}

Additions to the Math Calendar should be submitted at: bit.ly/1SDf5kF

To make suggestions for additions to other sections, and for full contact information, see www.ams.org/publications/ journals/notices/noticescontact

[Notices of the American Mathematical Society (ISSN 0002-9920) is published monthly except bimonthly in June/July by the American Mathematical Society at 201 Charles Street, Providence, RI 02904-2294 USA, GST No. 121892046 RT ${ }^{\star \star \star \star}$. Periodicals postage paid at Providence, RI, and additional mailing offices. POSTMASTER: Send address change notices to Notices of the American Mathematical Society, P.O. Box 6248, Providence, RI 02904-6248 USA.] Publication here of the Society's street address and the other bracketed information is a technical requirement of the U.S. Postal Service.

(c) Copyright 2017 by the American Mathematical Society. All rights reserved.

Printed in the United States of America. The paper used in this journal is acid-free and falls within the guidelines established to ensure permanence and durability.

Opinions expressed in signed Notices articles are those of the authors and do not necessarily reflect opinions of the editors or policies of the American Mathematical Society. 


\section{LETTERS TO THE EDITOR}

\section{Presidential Nomination by Petition?}

I recently received the September 2017 AMS Notices, which contains the information on the presidential candidates. Professors Pipher and Williams are eminently qualified, and whichever one is elected will no doubt be an excellent president.

Nevertheless I wonder if it would be appropriate to allow nomination by petition for AMS president (as it is now for other officers and the Council). This is the practice for many academic and professional organizations. I feel it would increase participation by the membership and allow for greater diversity. One could require a substantial number of supporting signatures to preclude frivolous nominations.

I urge the officers of the AMS as well as the president elect to set in motion a procedure for nomination by petition for president.

$$
\begin{array}{r}
\text {-Joe Auslander } \\
\text { Professor Emeritus of Mathematics } \\
\text { University of Maryland } \\
\text { jna@math. umd. edu }
\end{array}
$$

(Received September 8, 2017)

EDITOR'S NOTE. Voting in the current election closes November 3, 2017. See www . ams .org/about-us/ governance/elections/election-info.

\section{Travel Ban, Lincoln, and Beethoven}

Thank you for the story about mathematicians affected by the travel ban in the August 2017 Notices. I recently went to an LA Philharmonic concert where the program was Copland's Lincoln Portrait and Beethoven's Ninth Symphony. Lincoln Portrait features excerpts of Lincoln's speeches and writing, the first of which, from his 1862 message to Congress, is:

Fellow citizens, we cannot escape history. We of this congress and this administration will be remembered in spite of ourselves. No personal significance or insignificance can spare one or another of us. The fiery trial through which we pass will light us down in honor or dishonor to the latest generation. We, even we here, hold the power and bear the responsibility.

"We invite readers to submit letters to the editor to notices1etters@ams .org and post commentary on the Notices webpage www. ams.org/journals/notices.

For permission to reprint this article, please contact: reprint-permission@ams.org.
It was impossible not to think of the current administration. Following that, the "Ode to Joy" in Beethoven's Symphony became a prayer for peace and reconciliation for all mankind. I hadn't expected it, but the program seemed to be the orchestra's response to the question, "How do we, as musicians, respond to the catastrophic situation that confronts us?" That got me thinking: how do we respond, as mathematicians? I don't think this story about the travel ban was the full answer, but it is something. Thank you for that.

\section{- Lyla Fadali Occidental College}

(Received August 9, 2017)

\section{More to Internationalism than Migration}

Moon Duchin's timely commentary in the August 2017 Notices, paired with an article on the Travel Ban and another on a new Global Math Project, drew important attention to the importance of migration and international outreach in the history and current situation of American mathematical leadership. All three pieces, in their own ways, focus on the wealth of talent from foreign shores that Americans would stand to lose, whether through policies of exclusion or simply a failure to connect and encourage. They ask us to appreciate the great contributions from migrant mathematicians in the past and to imagine what future migrants may bring to our mathematical institutions.

My own research on the history of American mathematical internationalism suggests two corollary points to keep in mind that take our focus away from migrants and migration themselves. ${ }^{1}$ One is about the structural importance of an international outlook, regardless of whom it brings in from abroad. The other is a cautionary point about the politics of internationalism, and the need to keep a critical perspective on the rhetoric of openness.

While the stories of high-profile migrant mathematicians may be more visible, detailed recent research by historians (including myself) has shown that American internationalism required structural changes to the nation's mathematical institutions that may have been even more significant than migration itself for the mathematics profession, both domestically and internationally. Americans developed an internationally-oriented mathematical community led primarily by American-born mathematicians well before the famous influx of World War II refugees. (Indeed, L. E. Dickson, the subject of another piece in the

Barany1: See especially Michael J. Barany, Distributions in Postwar Mathematics, PhD dissertation, Princeton University, 2016, available at mbarany . com/publications . html. 
August Notices, was among the architects of this posture.) Convinced that the key to mattering in the discipline was to maintain strong ties to Europe, Americans approached everything from travel to publishing to the organization of research and teaching with Europe in mind. Toward the mid-twentieth century, American funding bodies and academic institutions prioritized international travel, international fellowships, and international publishing, including to sites beyond Europe. This redounded to mathematicians in the United States regardless of their countries of origin by helping forge vital connections and exchanges. The infrastructure required to look to the world made domestic mathematics stronger, beyond just what it enabled those from afar to contribute. When assessing the value of internationalism, it is therefore important to consider its homegrown beneficial effects, not just what it welcomes from abroad.

But we must be cautious about the values and priorities that get set aside in the name of internationalism. Tellingly, American apologists for Nazi Germany used the rhetoric of international understanding to excuse the latter regime's treatment of Jewish scholars. Just months before the German invasion of Poland, German mathematician Helmut Hasse confidently urged an American colleague to be "truly impartial and hence genuinely international" during an American debate over how to respond to Jewish mathematicians' dismissal from the Zentralblatt für Mathematik - a debate that led to the creation of the AMS's Mathematical Reviews. The American organizers of the 1950 International Congress of Mathematicians turned time and again to the language and ideals of internationalism and its associated impartiality, which helped them ignore their manifold shortcomings in including mathematicians of any political views from across the Iron Curtain and those with leftist views from the West. It also helped them dismiss the suggestion of awarding an invited address to Howard University's David Blackwell as a leading Black mathematician at an American HBCU, and their focus on mathematical fields and institutions meant they did not raise an eyebrow at the lack of women nominees for invited addresses. Throughout this history, American elites used a rhetoric of internationalism to claim the organizational forefront of the discipline while downplaying non-nation-based forms of discrimination and prejudice in mathematics.

Mathematicians should stand up for international values of inclusion, access, and exchange, which strengthen the discipline in many ways, both overt and subtle. At the same time, powerful members of the mathematical community must be responsible for recognizing that inclusion and exclusion come in many forms, and that internationalism should not be an end in itself. ${ }^{2}$

- Michael J. Barany

Dartmouth College mbarany.com

(Received August 10, 2017)

Barany2: Hat tip to the excellent new AMS inclusion/exclusion blog.

\section{Make March 20th Hypatia Day}

The death of Maryam Mizrakhani in July of this year again raised the question of the proper recognition of women in science.

Let me bring your attention to an online petition I initiated in March 2016 after publishing a paper that solves a 1,600-year old history puzzle. ${ }^{1}$

A famous female mathematician and astronomer, Hypatia, a daughter of Theon, lived in the fourth and fifth centuries CE in Alexandria. Her death (actually barbaric murder at the hands of a clique of Christian zealots) symbolized the transition from Antiquity to the Dark Middle Ages. The astronomical/calendrical background, related to the determination of Easter, is explained in my article in Vigiliae Christianae. ${ }^{2}$

Since, according to my reconstruction, Hypatia's last days were dedicated to finding the exact time for the vernal (spring) equinox, I propose remembering Hypatia annually on the day of the vernal equinox. By local time in Alexandria, the vernal equinox in the years 2016-2050 falls on the same date, March 20th, as that in $415 \mathrm{CE}$, the year of her murder. Therefore March 20th is an ideal date in our era to commemorate the first female astronomer and to celebrate women in science more generally. Let us make March 20th Hypatia Day!

-Ari Belenkiy

PhD Math, UC Irvine 1995

Vancouver BC, Canada ari.belenkiy@gmai1.com

(Received July 22, 2017)

\section{We Don't Need "so-called"}

To all editors, referees, and authors:

I have noticed an increase in use of "so-called" in many math papers. It can have a pejorative or at least disparaging sense, cf. the secondary dictionary meaning: "used to express one's view that a name or term is inappropriate." Examples include "so-called Islamic State," "so-called Caliphate,"' and "so-called leaders."

There is no need to use so-called in math papers. Authors can use italics, for example, operad. IFF the author really thinks something needs to be said, use "what is called" or "what is known as."

$$
\begin{array}{r}
\text {-Jim Stasheff } \\
\text { University of Pennsylvania and } \\
\text { University of North Carolina-Chapel Hill } \\
\text { jds@sas. upenn. edu }
\end{array}
$$

(Received July 3, 2017).

Belenkiy1: At the time of this writing, the petition has more than 900 signatures from 53 countries, representing all five continents. The petition is at https://www. change . org/p/canada-s-pari iament-commemorating-the-first-female-astronomerhypatia-of-alexandria

Belenkiy2: booksandjourna1s.bri11on1ine.com/content/journa1s/10.1163/15700720-12341264. 


\section{Support Women \\ Mathematics Scholars}

Make a gift to the Joan \& Joseph Birman Fellowship for Women Scholars

Your gift to the Joan \& Joseph Birman Fellowship for Women Scholars' endowment helps address the gender gap in mathematics by giving mid-career women scholars essential research support.

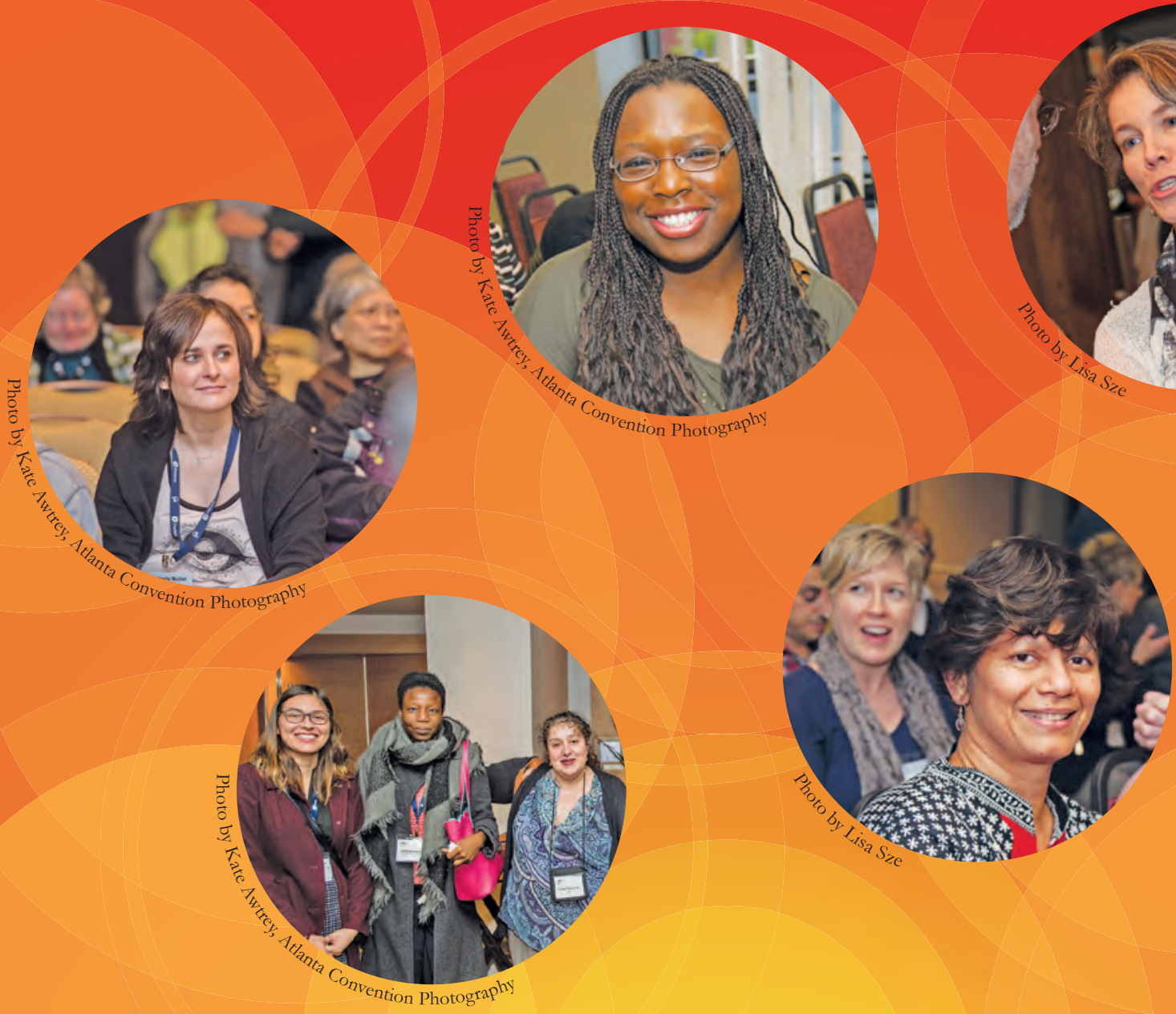

Visit www.ams.org/support to learn more and give!

Contact the AMS Development Office by phone: 401-455-4111 or email: development@ams.org 


\section{Antibiotics Time Machines Are Hard to Build}

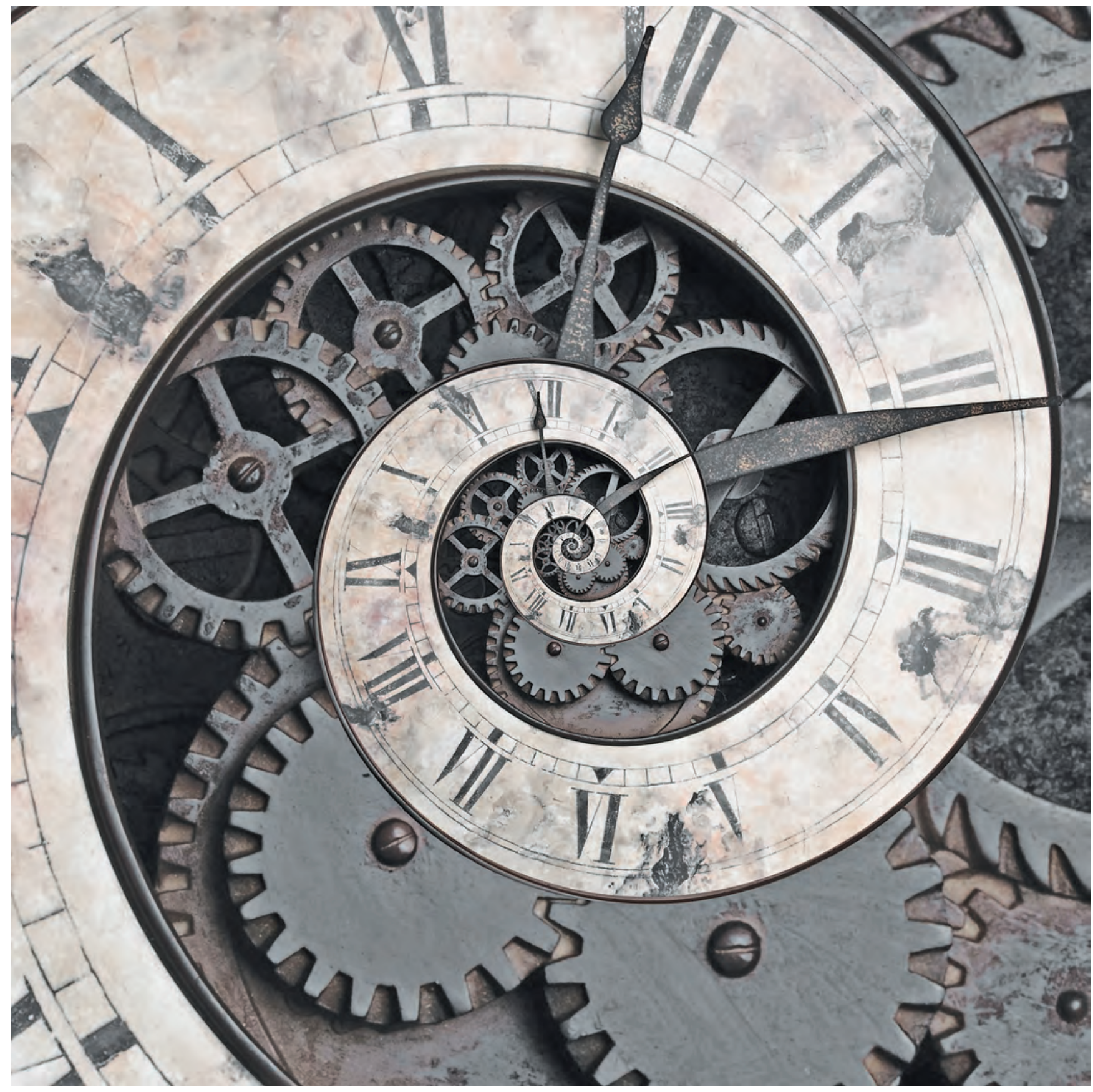

Ngoc M. Tran and Jed Yang

Communicated by Daniel J. Velleman 
ABSTRACT. Could we somehow go back in time before bacteria developed resistance to antibiotics? Mira et al. famously cast antibiotics resistance as an optimization problem called the "antibiotics time machine." We show that such a strategy is NP-hard to compute.

\section{Antibiotics Time Machines}

Resistance to antibiotics in bacteria is a central problem of modern medicine. The work of Mira et al. [MCG $\left.{ }^{+} 15\right]$, a group of biologists and mathematicians, cast undoing or avoiding antibiotics resistance as an optimization problem, dubbed the "antibiotics time machine." Their novel approach received wide press coverage. In this article, we define such a time machine mathematically, show that such strategies are NP-hard to compute, and discuss the implications.

Let us think of an antibiotic as a sieve: it kills bacteria with a certain genotype. Repeated applications of the same antibiotic result in a population without this genotype, which renders the antibiotic ineffective. Furthermore, the course of antibiotics has altered the distribution of genotypes, or, in biological terms, the fitness landscape, of our bacterial species. This is a critical problem: imagine that the pre-antibiotics bacterial population is held in equilibrium by various factors, such as natural predators, competition for resources, and so on. A new fitness landscape disrupts this equilibrium, potentially leading to a population explosion of these bacteria. One way to mitigate these consequences is to rotate the use of antibiotics that target the same genes but select for different genotypes.

Mira et al. [MCG $\left.{ }^{+} 15\right]$ considered the following model. Suppose we have a population of bacteria, all of the same unmutated genotype (called the "wild type"). We are given a set of antibiotics. For each bacterium of a given type, an antibiotic mutates it to another type with some known probability. The problem is to find a sequence of antibiotics (called a treatment plan) that maximizes the fraction of bacteria returning to the wild type. This optimal plan is called an antibiotic time machine by Mira et al., alluding to the idea of reversing unnecessary mutations induced in the bacterial population through antibiotic treatments.

Here is a concrete description of the model. Let $S=\{1,2, \ldots, d\}$ be a set of $d$ states, where each state is a bacterial genotype. A distribution of bacterial population

Ngoc Mai Tran is assistant professor of mathematics at the University of Texas at Austin and $W$-2 professor (Bonn Junior Fellow) at the Hausdorff Center for Mathematics, Germany. Her email address is ntran@math. utexas . edu.

Jed Yang is visiting assistant professor of computer science at Carleton College. His email address is jyang@car leton. edu.

For permission to reprint this article, please contact:

reprint-permission@ams.org.

DOI: http://dx.doi.org/10.1090/noti1590 is a vector in the standard $(d-1)$-dimensional simplex $\Delta_{d-1}=\left\{\left(x_{1}, x_{2}, \ldots, x_{d}\right) \in \mathbb{R}^{d} \mid \sum_{i=1}^{d} x_{i}=1\right.$ and $x_{i} \geq$ 0 for all $i\}$, where for $\mathbf{s}=\left(\mathbf{s}_{1}, \mathbf{s}_{2}, \ldots, \mathbf{s}_{d}\right) \in \Delta_{d-1}, \mathbf{s}_{i}$ represents the fraction of the population with genotype $i \in S$. A $d \times d$ matrix is a transition matrix if each entry belongs to the interval $[0,1]$ and each row sums to 1 . Let $\mathcal{T}$ be a finite set consisting of $K$ transition matrices, each corresponding to the effect of a given antibiotic on the genotypes of the bacterial population. That is, if $T=\left(t_{i j}\right)$ is the transition matrix corresponding to some antibiotic, then $t_{i j}$ is the probability that a single bacterium of genotype $i$ will have genotype $j$ after being treated with the antibiotic. As our population consists of millions of bacteria, we can assume that the population evolves deterministically; that is, $t_{i j}$ can be seen as the fraction of bacteria that go from genotype $i$ to $j$ after treatment. Thus, if the population distribution is $\mathbf{s} \in \Delta_{d-1}$ and we apply an antibiotic with transition matrix $T$, then the population distribution after treatment will be $\mathbf{s} T$.

Let $\mathbf{s}, \mathbf{t} \in \Delta_{d-1}$ be the starting and targeted population distributions, respectively. Let $N \in \mathbb{N}$ be the length of the treatment plan. The time machine $\mathcal{T} \mathcal{M}(d, K, N)$ is the solution to the following optimization problem:

$$
\begin{array}{ll}
\text { maximize } & \mathbf{s} T_{1} T_{2} \cdots T_{N} \mathbf{t}^{\top} \\
\text { subject to } & T_{1}, \ldots, T_{N} \in \mathcal{T} .
\end{array}
$$

As a concrete example, suppose $\mathbf{s}=\mathbf{t}=(1,0, \ldots, 0) \in$ $\Delta_{d-1}$. Then $\mathbf{s} T_{1} T_{2} \cdots T_{N} \mathbf{t}^{\top}$ is the fraction of bacteria that end up as the wild type after the application of $N$ antibiotics. Our goal is to choose a sequence of antibiotics that will maximize this number.

\section{Complexity of the Time Machine}

How do we solve this problem numerically? Mira et al. $\left[\mathrm{MCG}^{+} 15\right]$ used brute force: they enumerated all possible treatment plans. With $K$ antibiotics, there are $K^{N}$ many treatment plans of length $N$, so this algorithm is not efficient. Mira et al. raised the question of whether an efficient algorithm exists. Unfortunately, we show that the problem is NP-hard; that is, there is likely no polynomial-time algorithm for solving such a problem.

Theorem 1. The time machine optimization problem in (1) is NP-hard.

We first consider an easier decision problem: for a given threshold $\alpha \in(0,1]$, decide whether there exists a treatment plan of length $N$ such that

$$
\begin{gathered}
\mathbf{s} T_{1} T_{2} \cdots T_{N} \mathbf{t}^{\top} \geq \alpha \\
\text { subject to } T_{1}, \ldots, T_{N} \in \mathcal{T} .
\end{gathered}
$$

Clearly if one can solve (1) in polynomial time, then one can also solve (2) in polynomial time. Our main result states that the latter problem is NP-hard, and thus Theorem 1 follows as a corollary.

Theorem 2. The time machine decision problem in (2) is NP-hard. 
Our proof relies on reducing the 3-satisfiability problem (3-SAT) to a special instance of (2) where $\alpha=1$. Since 3-SAT is NP-hard, we have that (2) is also. Biologically, this means that there are certain inputs where computing an exact solution is likely very time consuming. Furthermore, if $\alpha$ is rational, we may modify our construction to first "discard" a $(1-\alpha)$-fraction of the population, so (2) is hard for infinitely many values of $\alpha$. We provide the details in the appendix.

\section{Robots and Bacteria}

Optimization problems like the time machine also arise in large-scale robotics control. One can equate each bacterium to a robot; each genotype is one of $d$ possible states (such as standing or walking), and each antibiotic is an instruction which changes each robot's state independently at random. The goal is to get all robots to the same state. The difficulty is that one instruction is broadcast to the whole robot population, and thus one cannot optimally control each individual robot. Indeed, if there were only one bacterium instead of a population, then one could apply an antibiotic, check the new state of the bacterium, and repeat. In this case, the time machine is a Markov decision process (MDP) on $d$ states, which can be solved by dynamic programming. When there is a population of bacteria, the population state is a point in the standard $(d-1)$-dimensional simplex $\Delta_{d-1}$, so our MDP has uncountably many states.

In the optimal control literature, our problem is an instance of a finite-horizon, unobservable MDP with belief-dependent, time-dependent reward function. This is a controlled stochastic system on finitely many states $S$, with finitely many possible actions $\mathcal{A}$. At discrete time $t=0,1, \ldots$, the system is assumed to be in some true but unknown state, and the controller sees only a belief distribution $P_{t}$ over the set of states $S$. Based on $P_{t}$, the controller can select an action $a \in \mathcal{A}$, which transits the system to time step $t+1$ with belief distribution $P_{t+1}=f\left(P_{t}, a\right)$ and gives reward $r_{t}\left(P_{t+1}\right)$. A policy is a sequence of actions. In finite-horizon problems, the goal is to choose a policy that maximizes the total expected reward after $T$ time steps. In the antibiotics context, a state is a genotype, $P_{t}$ is the distribution of genotypes in the bacterial population at time $t$, and an action is an antibiotic. The reward function $r_{t}$ is identically zero for $t<T$, while $r_{T}\left(P_{T+1}\right)=\left(P_{T+1}\right)_{1}$. Finite-horizon unobservable MDPs are known to be NP-hard [LGM01], with a reduction to $k$-SAT similar to our proof of Theorem 2 .

\section{How to Build a Time Machine Anyway}

Exact algorithms for unobservable MDPs use incremental pruning, and this idea also yields an exact algorithm for the antibiotics time machine. The premise is to enumerate by brute force but eliminate suboptimal treatment plans early. To illustrate, suppose we are able to solve the antibiotics time machine with $N=1$ for each possible starting state $\mathbf{s} \in \Delta_{d-1}$. Let $T$ be an antibiotic that does not achieve the maximum in (1) for any $\mathbf{s}$. Then for the antibiotics time machine with any $N \geq 1$ and any starting point, the optimal treatment plan cannot end with $T$. If there are $K^{\prime}$ such suboptimal antibiotics, then the total number of treatment plans to consider is now $K^{T-1}\left(K-K^{\prime}\right)$, as opposed to $K^{T}$. Repeated application of this argument for $N=2,3, \ldots$ amounts to enumerating the treatment plans in backward time steps while discarding suboptimal branches early.

One can also save computation time by restricting or approximating the belief space. Let us again consider the time machine in one step. As a function of the starting state $\mathbf{s}$, the objective function in (1) is the maximum of $K$ linear functions, so it is convex and piecewise linear. Its graph subdivides $\Delta_{d-1}$ into at most $K$ fulldimensional polytopes, where all starting points $\mathbf{s}$ in the same polytope are optimized by the same antibiotic unique to that polytope. Now, suppose we know that $\mathbf{s}$ can only lie in some strict subset $\Delta^{\prime}$ of $\Delta_{d-1}$. If $T$ is an antibiotic whose polytope does not intersect $\Delta^{\prime}$, then $T$ is also suboptimal. Combined with pruning, this reduces the set of possible optimal treatment plans even further.

\section{Practical Considerations: The Efficient, Approximate Time Machine?}

Real-life instances of the time machine make exact computations prohibitive. To put the problem scale into perspective, the particular time machine of Mira et al. $\left[\mathrm{MCG}^{+} 15\right]$ had 16 genotypes and 15 antibiotics and used the brute force algorithm up to $N=6$, that is, over $15^{6} \approx 1.1 \times 10^{7}$ treatment plans. In comparison, the methicillin-resistant Staphylococcus aureus (MRSA), a "superbug" common in healthcare centers, has about 30 genotypes identified $\left[\mathrm{SHIB}^{+} 06\right]$. Currently there are six antibiotics recommended for treatment of MRSA. A standard therapy is 28 days, with antibiotics applied between one and three times a day. Altogether this gives a time machine problem with $d \approx 30, K \approx 5$ to 10 , and $N \approx 30$ to 90 . The smallest instance with $(d, K, N)=(30,5,30)$ already creates $5^{30} \approx 9.3 \times 10^{20}$ possible treatment plans. Unless the transition matrices have very special structures, such instances cannot be solved exactly.

another significant
contribution of
mathematics to
society

This is where connections to robotics could be very valuable. Unobservable MDPs are special instances of partially observable MDPs (POMDPs), which find extensive applications in robotics and motion planning [DS13]. Large-scale approximation algorithms for these problems use a combination of belief space approximation and early pruning of suboptimal policies, as discussed above. However, their reward functions are not time or belief dependent. If such approximation algorithms can be adapted to the antibiotics time machine, one could solve real-life instances with tens of thousands of states and antibiotics. That would be yet another significant contribution of mathematics to society, one that even has a cool name. 


\section{Appendix A. Reduction Construction and Proof of the Main Theorem}

Let $X=\left\{x_{1}, \ldots, x_{n}\right\}$ be a set of Boolean variables taking Boolean values $\{+,-\}$. A triple $\left(\varepsilon_{i} x_{i}, \varepsilon_{j} x_{j}, \varepsilon_{k} x_{k}\right)$ with $\varepsilon_{i}, \varepsilon_{j}, \varepsilon_{k} \in\{+,-\}$ is called a clause. Let $C=\left\{c_{1}, \ldots, c_{m}\right\}$ be a set of clauses. Write $\left(x_{i}=v_{i}\right)_{i}$ to mean $x_{i}=v_{i}$ for $i=1, \ldots, n$. An assignment $\left(x_{i}=v_{i}\right)_{i}$ satisfies a clause $c=\left(\varepsilon_{i} x_{i}, \varepsilon_{j} x_{j}, \varepsilon_{k} x_{k}\right)$ if $\left(\varepsilon_{i}, \varepsilon_{j}, \varepsilon_{k}\right) \neq\left(v_{i}, v_{j}, v_{k}\right)$. The 3-SAT problem is: given a set of variables and clauses, decide whether there exists an assignment that satisfies all clauses. We will assume that each variable occurs (negated or not) in at least one clause. In this section, we reduce a 3-SAT instance to an instance of (2) by constructing an appropriate time machine.

\section{Construction of the Time Machine}

Let $N=m+2$ and $\alpha=1$. Consider a time machine with $d=3 n+m+3$ states and $K=7 m+2$ transition matrices. Index these $d$ states as follows: For each of the $n$ variables $x \in X$, there are states $\mathbf{x}, \mathbf{x}^{-}$, and $\mathbf{x}^{+}$. For each of the $m$ clauses $c \in C$, there is a state c. Finally, the last three states are the start state $\mathbf{s}$, the "death" state $\mathbf{d}$, and the "tally" state $\mathbf{f}$. We identify each state with its characteristic (row) vector and define each transition matrix by its action on these basis vectors.

If $c=\left(\varepsilon_{i} x_{i}, \varepsilon_{j} x_{j}, \varepsilon_{k} x_{k}\right) \in C$, then for each choice of $\left(v_{i}, v_{j}, v_{k}\right) \in\{+,-\}^{3} \backslash\left\{\left(\varepsilon_{i}, \varepsilon_{j}, \varepsilon_{k}\right)\right\}$, define a transition matrix $T=T_{\mathcal{C}}^{v_{i}, v_{j}, v_{k}}$ as follows:

$$
\mathbf{z} T= \begin{cases}\mathbf{d}, & \mathbf{z}=\mathbf{s}, \\ \mathbf{f}, & \mathbf{z}=\mathbf{c}, \\ \mathbf{x}_{\ell}^{v_{\ell}}, & \mathbf{z}=\mathbf{x}_{\ell}, \ell \in\{i, j, k\}, \\ \mathbf{d}, & \mathbf{z}=\mathbf{x}_{\ell}^{-v_{\ell}}, \ell \in\{i, j, k\}, \\ \mathbf{z}, & \text { otherwise. }\end{cases}
$$

Define a starting matrix $S$ by

$$
\mathbf{z} S= \begin{cases}p\left(\sum_{x \in X} \mathbf{x}+\sum_{c \in C} \mathbf{c}\right), & \mathbf{z}=\mathbf{s}, \\ \mathbf{d}, & \text { otherwise, }\end{cases}
$$

where $p=1 /(n+m)$. Lastly, define a final matrix $F$ by

$$
\mathbf{z} F= \begin{cases}\mathbf{s}, & \mathbf{z} \in\left\{\mathbf{x}_{i}^{v_{i}}: v_{i} \in\{+,-\}\right\}, \\ \mathbf{s}, & \mathbf{f}, \\ \mathbf{d}, & \text { otherwise. }\end{cases}
$$

Let $\mathcal{T}$ consist of $S, F$, and the $7 m$ transition matrices $T_{c}^{v_{i}, v_{j}, v_{k}}$ defined above. This concludes the reduction construction.

\section{Proof of Reduction}

We shall prove that with the above time machine constructed from the 3-SAT problem, (2) solves the 3-SAT problem with the given clauses. Since the latter is NP-hard, this implies that (2) is also. We refer to the fraction of the bacterial population in a given state as its weight. First, suppose $\left(x_{i}=v_{i}\right)_{i}$ is a satisfying assignment. Apply $S$ to $\mathbf{s}$. For each $c=\left(\varepsilon_{i} x_{i}, \varepsilon_{j} x_{j}, \varepsilon_{k} x_{k}\right) \in C$, apply $T_{c}^{v_{i}, v_{j}, v_{k}}$, which exists as $c$ is satisfied. Finally, apply $F$.
Lemma 3. The sequence of matrices given above sends all weight back to $\mathbf{s}$. That is, it is a solution of (2) for $\alpha=1$.

Proof. For each $x \in X$, the matrix $S$ sends some weight to $\mathbf{x}$. The weight is moved to $\mathbf{x}^{v_{i}}$ the first time $\pm x$ occurs in a clause $c$ and is moved back to $\mathbf{s}$ by $F$ at the end. For each $c \in C$, the matrix $S$ sends some weight to c. This weight is moved to $\mathbf{f}$ by $T_{\mathcal{C}}^{v_{i}, v_{j}, v_{k}}$ and is moved back to $\mathbf{s}$ by $F$ at the end. Therefore all the weights return to $\mathbf{s}$, as desired.

Conversely, suppose there is a sequence $T_{1}, T_{2}, \ldots, T_{N}$ of transition matrices so that

$$
\mathbf{s} T_{1} T_{2} \cdots T_{N} \mathbf{t}^{\top}=1 .
$$

The aim is to extract a satisfying assignment.

Consider the process of applying the transition matrices $T_{i}$ to $\mathbf{s}$ sequentially in $N$ steps. Note that any weight at $\mathbf{d}$ stays at $\mathbf{d}$ forever. As such, to achieve full weight at $\mathbf{s}$ after $N$ steps, the state $\mathbf{d}$ cannot receive weight at any point in the process. ${ }^{1}$ Consequently, it is clear that the first matrix to apply has to be $S$, as we have $T(\mathbf{s})=\mathbf{d}$ for $T \in \mathcal{T} \backslash\{S\}$.

The only way for $\mathbf{s}$ to gain weight is to apply $F$. Prior to applying $F$ for the first time, all weights must be supported on the $\mathbf{x}_{i}^{ \pm}$and $\mathbf{f}$, so as to avoid losing any weight to the death state $\mathbf{d}$. In particular, for each clause $c=\left(\varepsilon_{i} x_{i}, \varepsilon_{j} x_{j}, \varepsilon_{k} x_{k}\right) \in C$, state $\mathbf{c}$ must no longer carry weight at this point. That is, after $\mathbf{c}$ receives some weight by $S$ in the first step, it must subsequently lose the weight, which can only be achieved by applying an associated matrix $T_{c}^{v_{i}, v_{j}, v_{k}}$ for some choice of $\left(v_{i}, v_{j}, v_{k}\right)$. This takes (at least) one step for each clause. Since the sequence of matrices is of length precisely $N=m+2$, we conclude that the sequence starts with $S$, finishes with $F$, and contains precisely one matrix corresponding to each clause.

When $S$ is applied, the full weight at $\mathbf{s}$ is split into $n+m$ packets, each of weight $p=1 /(n+m)$. Since no other matrices split weights, we may consider the remaining process as a discrete system moving each packet as a unit. We already saw that the $m$ packets associated to the clauses are moved to $\mathbf{f}$ during the middle $m$ steps. It remains to analyze the remaining $n$ packets associated to the variables. To avoid moving any weight to the death state $\mathbf{d}$, the packet at $\mathbf{x}_{i}$ can only be moved to $\mathbf{x}_{i}^{+}$or $\mathbf{x}_{i}^{-}$. Once this happens, the packet can only be moved again by $F$ at the last step. So at the penultimate step, there is a packet on $\mathbf{x}_{i}^{\tilde{v}_{i}}$ for exactly one choice of $\tilde{v}_{i}$ for each $i$. The following lemma finishes the proof.

Lemma 4. For each $i$, let $\tilde{v}_{i}$ be such that $\mathbf{x}_{i}^{\tilde{v}_{i}}$ has nonzero weight after $m+1$ steps, i.e.,

$$
\mathbf{s} T_{1} T_{2} \cdots T_{N-1}\left(\mathbf{x}_{i}^{\tilde{v}_{i}}\right)^{\top}>0 .
$$

Then $\left(x_{i}=\tilde{v}_{i}\right)_{i}$ is a satisfying assignment.

${ }^{1}$ Intuitively, $\mathbf{d}$ is a "death" state, where bacteria go to die, never to be recovered to the wild type. 
Indeed, consider a clause $c=\left(\varepsilon_{i} x_{i}, \varepsilon_{j} x_{j}, \varepsilon_{k} x_{k}\right) \in C$. We know that (exactly) one associated transition matrix $T_{C}^{v_{i}, v_{j}, v_{k}}$ is used. Suppose, towards a contradiction, that $\tilde{v}_{\ell} \neq v_{\ell}$ for some $\ell \in\{i, j, k\}$. After applying $T_{c}^{v_{i}, v_{j}, v_{k}}$, the packet corresponding to $x_{\ell}$ is at $\mathbf{x}_{\ell}^{v_{\ell}}$ or $\mathbf{d}$, with no way of moving to $\mathbf{x}_{\ell}^{\tilde{v}_{\ell}}$, a contradiction. So $\left(\tilde{v}_{i}, \tilde{v}_{j}, \widetilde{v}_{k}\right)=$ $\left(v_{i}, v_{j}, v_{k}\right) \neq\left(\varepsilon_{i}, \varepsilon_{j}, \varepsilon_{k}\right)$ by construction, implying that clause $c$ is satisfied.

This shows that a 3-SAT instance has a solution if and only if the associated time machine can attain a threshold of 1 . We therefore conclude that the time machine decision problem is NP-hard, as desired.

\section{Acknowledgments}

The authors wish to thank Bernd Sturmfels and Joel Kileel for stimulating discussions. The first-named author is supported by an award from the Simons Foundation (\#197982 to The University of Texas at Austin). The second-named author is supported by NSF RTG grant NSF/DMS-1148634.

\section{References}

[DS13] AlAin Dutech and Bruno ScherRer, Partially observable markov decision processes, Markov Decision Processes in Artificial Intelligence, ISTE, London, 2010, pp. 187-227. MR 2808773

[LGM01] CHRISTOPHER LUSENA, JUDY GOLDSMITH, and MARTIN MUNDHENK, Nonapproximability results for partially observable Markov decision processes, J. Artificial Intelligence Res. 14 (2001), no. 1, 83-113. MR 1835269

$\left[\mathrm{MCG}^{+} 15\right]$ Portia M. Mira, Kristina Crona, Devin Greene, JuAn C. MEZA, BERnd STURMFEls, and Miriam BARLOw, Rational design of antibiotic treatment plans: A treatment strategy for managing evolution and reversing resistance, PLOS ONE 10 (2015), no. 5, e0122283.

[SHIB ${ }^{+}$06] AleX J. Stephens, Flavia Huygens, John InMANBAmber, ERIN P. Price, Graeme R. Nimmo, JACQUELINE SCHOONEVELDT, WENDY MUNCKHOF, and PHILIP M. GIFFARD, Methicillin-resistant Staphylococcus aureus genotyping using a small set of polymorphisms, J. of Medical Microbiology 55 (2006), no. $1,43-51$.

\section{Photo Credits}

Opening photo courtesy of Getty Images. Photo of Ngoc Mai Tran courtesy of Barbara Frommann. Photo of Jed Yang courtesy of Jed Yang.

\section{ABOUT THE AUTHORS}

Ngoc Mai Tran loves to bring new applications to tropical geometry and probability. She also likes manga, strategy games, and traveling.

Jed Yang enjoys applying computational complexity techniques to questions from various disciplines. In his spare time, he likes to think about mathematics.

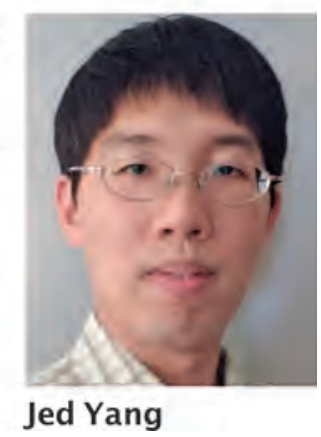




\section{Call For Suggestions}

\section{YOUR SUGGESTIONS ARE WANTED BY:}

the Nominating Committee, for the following contested seats in the 2018 AMS elections:

vice president, trustee, and five members at large of the Council.

Deadline for suggestions: November 1, 2017

the President, for the following contested seats in the $\mathbf{2 0 1 8}$ AMS elections:

three members of the Nominating Committee and two members of the Editorial Boards Committee.

Deadline for suggestions: January 31, 2018

the Editorial Boards Committee, for appointments to various editorial boards of AMS publications.

Deadline for suggestions: Can be submitted any time

Send your suggestions for any of the above to:

Carla D. Savage, Secretary

American Mathematical Society

Department of Computer Science

North Carolina State University

Raleigh, NC 27695-8206 USA

secretary@ams.org

or submit them online at www.ams.org/committee-nominate 


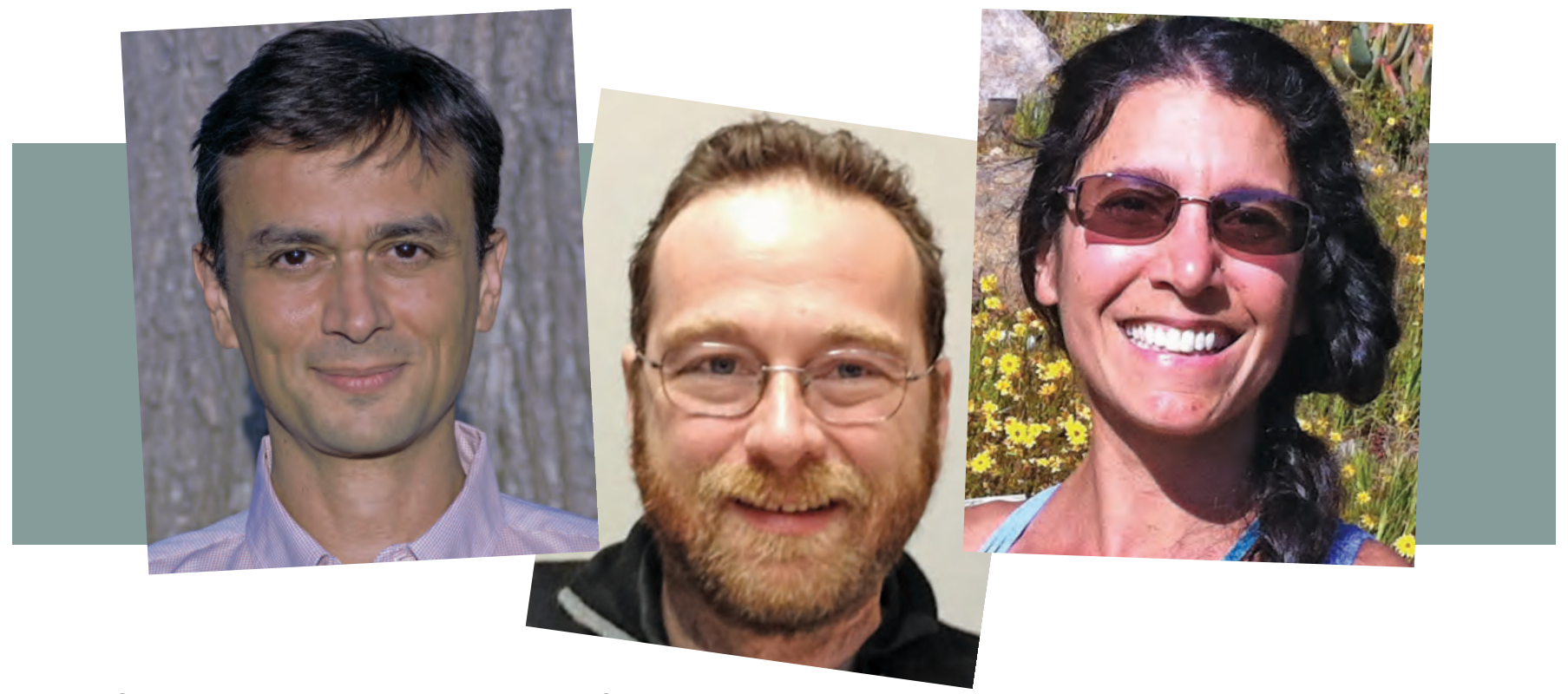

From left to right: Paul Balmer, Pavel Etingof, Monica Vazirani.

In this sampler, the speakers above have kindly provided introductions to their Invited Addresses for the upcoming AMS Fall Western Sectional Meeting.

\section{November $4-5,2017$ \\ (Saturday-Sunday) \\ University of California, Riverside \\ Riverside, CA}

An Invitation to Tensor-Triangular Geometry by Paul Balmer (UCLA)

page 1143

Double Affine Hecke Algebras and Their Applications by Pavel Etingof (MIT)

page 1144

Combinatorics, Categorification, and Crystals by Monica Vazirani (UC, Davis) page 1145

For permission to reprint this article, please contact: reprint-permission@ams . org.

DOI: http://dx.doi.org/10.1090/noti1591

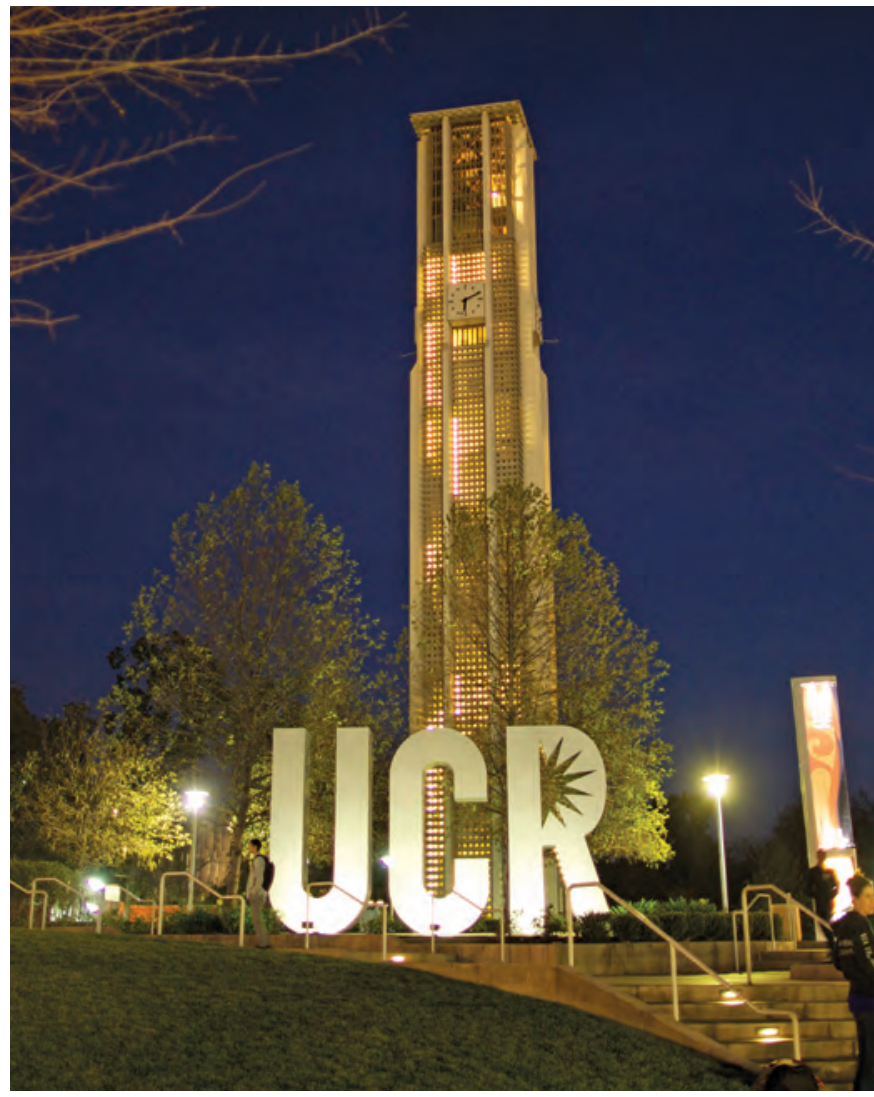




\section{AMS FALL WESTERN SECTIONAL SAMPLER}

\section{An Invitation to Tensor-Triangular Geometry}

\section{by Paul Balmer}

Tensor-triangulated categories appear in topology, in algebraic geometry, in representation theory, and in the theory of $C^{*}$-algebras for instance. The aim of tensortriangular geometry is to develop a unified treatment of this broad variety of examples. By way of introduction to this advanced topic, let us discuss an example in homotopy theory based on joint work [2] with Beren Sanders.

As a warm-up, consider a finite group $G$ acting on a finite set $X$. For every subgroup $H$ of $G$ we can count the number of fixed points $\left|X^{H}\right|$ of $X$ under the action of $H$. This number depends on $H$ only up to conjugacy in $G$. Moreover, if $p$ is a prime number, then $\left|X^{H}\right|$ is congruent modulo $p$ to the number $\left|X^{K}\right|$ for any normal subgroup $K<H$ of index $p$. For illustration, consider the extreme case of the cyclic group $G=C_{p}$ of order $p$, with $K=1$ trivial and $H=G$. What we are saying is that the number of elements in the finite set $X$ is congruent modulo $p$ to the number of fixed points $\left|X^{G}\right|$; indeed the difference $X-X^{G}$ consists of $G$-orbits which all have $p$ elements. For a general group $G$, if one can move from the subgroup $H$ to the subgroup $K$ via a tower of index- $p$ normal subgroups and conjugation in $G$, then the number $\left|X^{H}\right|$ will be congruent modulo $p$ to the number $\left|X^{K}\right|$ independently of the chosen $G$-set $X$.

Building on the above basic combinatorics, Dress [3] described the Zariski spectrum of prime ideals of the socalled Burnside ring $A(G)$. One can add and multiply finite $G$-sets by taking disjoint union and product. The commutative ring $A(G)$ is obtained from this by formally including opposites for addition. Taking $H$-fixed points defines a ring homomorphism $f^{H}: A(G) \rightarrow \mathbb{Z}$ from the Burnside ring to the ring of integers $\mathbb{Z}=A(1)$. Pulling back ordinary prime ideals of $\mathbb{Z}$ via $f^{H}$, we obtain prime ideals in $A(G)$. Dress's theorem states that this construction catches all the prime ideals of the Burnside ring. The congruence discussed in our warm-up now becomes the following fact: If $H$ and $K$ are related as above (by towers of index- $p$ subgroups and conjugacy) for some prime $p$, then the prime ideal of the Burnside ring pulled back from the prime $p \mathbb{Z}$ via $f^{H}$ coincides with the one pulled back via $f^{K}$. This collision is the only redundancy among primes in the Burnside ring in terms of primes of $\mathbb{Z}$ and conjugacy classes of subgroup $H$. For instance, the spectrum of $A\left(C_{p}\right)$ is given in Figure 1 , in which $p(H, q)$ denotes the pulled-back prime $\left(f^{H}\right)^{-1}(q \mathbb{Z})$ for the two available subgroups $H=1$ and $H=$ $C_{p}$. The green dots form the copy of $\operatorname{Spec}(\mathbb{Z})$ pulled back via $f^{1}$ ("forget the action"), and the red dots form the copy of $\operatorname{Spec}(\mathbb{Z})$ pulled back via the $G$-fixed points homomorphism $f^{G}$. The collision happens here at a single green-red point.

Paul Balmer is professor of mathematics at UCLA. His e-mail address is balmer@math.ucla.edu.

For permission to reprint this article, please contact: reprint-permission@ams.org.

DOI: http://dx.doi.org/10.1090/noti1594

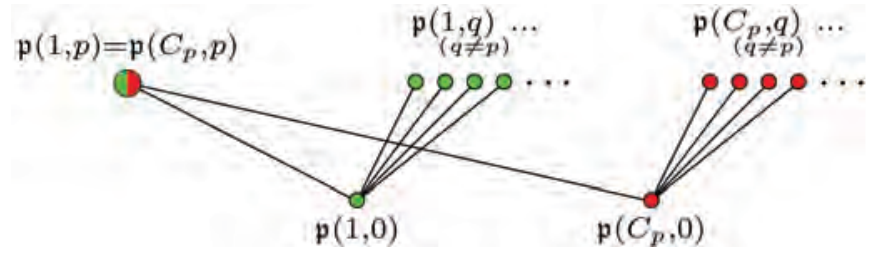

Figure 1. The Zariski spectrum of the Burnside ring $A\left(C_{p}\right)$. The pullbacks collide at the single green-red point.

Let us now ask a similar question, replacing finite $G$-sets with topological spaces, on which our finite group $G$ acts continuously. The problem has thus gained immense complexity due to the wild class of such available $G$-spaces. To handle this complexity, we pass to suitable stable homotopy categories. In doing so, the prime ideals of commutative algebra will be replaced by a sophisticated notion of primes. In the nonequivariant case, that is, for $G=1$, Hopkins and Smith [4], building on earlier work with Devinatz, established the celebrated chromatic filtration in stable homotopy theory. Consider the Spanier-Whitehead category $\mathrm{SH}^{c}$ of finite pointed CW-complexes, up to homotopy and up to stabilization, i.e., after formally inverting suspension. This $\mathrm{SH}^{c}$ is a tensor-triangulated category; i.e., it comes with a tensor product (smash product) and with exact triangles (Puppe sequences). Given a prime number $p$, one can localize this Spanier-Whitehead category at $p$ by inverting all other primes. This produces the $p$-local stable homotopy category of finite spectra. Hopkins and Smith's theorem asserts that finite $p$-local spectra are essentially classified by a number, the so-called ( $p$-local) chromatic height, which can be defined in terms of Morava $K$-theories. The chromatic height of $X$ is greater than or equal to the chromatic height of $Y$ if and only if $X$ can be constructed out of $Y$ by means of the available tensor-triangular operations: cones, direct summands, suspension, etc.

In tensor-triangular geometry one considers prime subcategories of a tensor-triangulated category $T$ in an analogous way to the prime ideals of a commutative ring in commutative algebra. Taken together, these tensor-triangular primes form a space, $\operatorname{Spc}(T)$, called the tensor-triangular spectrum of $T$. The topological version of Dress's result for the Burnside ring can now be phrased in terms of the tensor-triangular spectrum of $\mathrm{SH}(G)^{c}$, the tensor-triangulated category of finite $G$-spectra. The latter is a $G$-equivariant analogue of the category $\mathrm{SH}^{c}$ studied by Hopkins and Smith. For every subgroup $H$ of $G$, we have a geometric $H$-fixed points functor $F^{H}$ from $\mathrm{SH}(G)^{c}$ to $\mathrm{SH}^{c}$, and very much as in the Dress case we can pull back tensortriangular primes from $\mathrm{SH}^{c}$ to $\mathrm{SH}(G)^{c}$ via $F^{H}$ and obtain $G$-equivariant tensor-triangular primes denoted $P(H, p, n)$ for every subgroup $H$, prime number $p$, and chromatic height $n$. Our theorem [2] is that these $P(H, p, n)$ are all the tensor-triangular primes of $\operatorname{SH}(G)^{c}$. Moreover, the collision observed by Dress is resolved, in that $P(H, p, n)$ and $P(K, p, n)$ are now distinct when $K<H$ is a normal 


\section{AMS FALL WESTERN SECTIONAL SAMPLER}

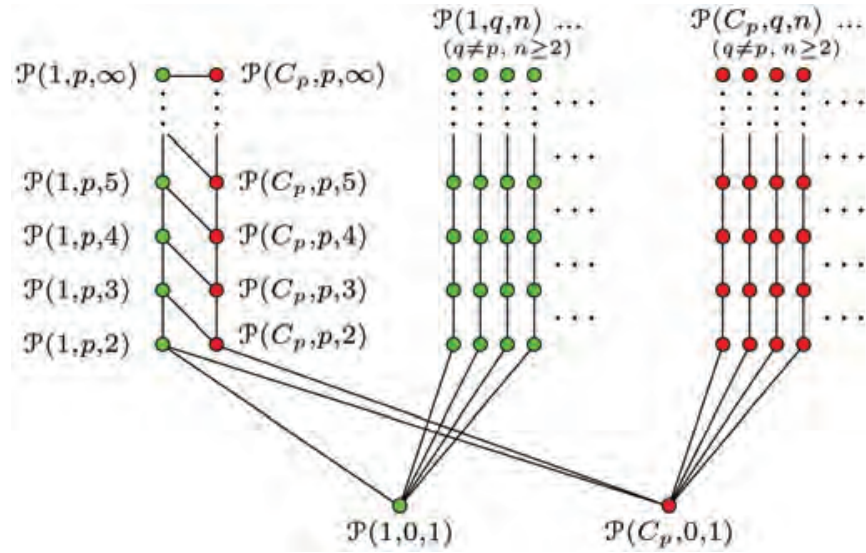

Figure 2. The tensor-triangular spectrum of $\mathrm{SH}\left(C_{p}\right)^{C}$. The point $P(1, p, n)$ does not equal $P\left(C_{p}, p, n\right)$ but does at least sit in the closure of the point $P\left(C_{p}, p, n-1\right)$.

subgroup of index $p$. However, something survives of the Dress collision, in that the point $P(K, p, n+1)$ now sits in the closure of the point $P(H, p, n)$. The spectrum $\operatorname{Spc}\left(S H(G)^{c}\right)$ is illustrated for the special case of $G=C_{p}$ in Figure 2. Here the green and red dots are the images of the Hopkins-Smith chromatic primes under $F^{1}$ (forget the action) and $F^{G}$ (geometric fixed points) respectively.

From the determination of the tensor-triangular spectrum, one can always classify all objects up to the available tensor-triangular operations. The resulting equivariant chromatic filtration in the case of $\mathrm{SH}(G)^{c}$ can also be found in [2]. Such a classification of all objects up to the available tensor-triangular operations is the best classification one can hope for, because most tensortriangulated categories we come across are wild. These ideas go way beyond (equivariant) homotopy theory, for example, to algebraic geometry, representation theory, and the theory of motives. The aim of tensor-triangular geometry is to develop a unified treatment of this broad variety of examples. For an introduction, see my article on "Tensor triangular geometry" [1].

\section{References}

[1] PAUl BALmer, Tensor triangular geometry, Proceedings of the International Congress of Mathematicians, Volume II, Hindustan Book Agency, New Delhi, 2010, pp. 85-112. MR 2827786

[2] PAul BAlmer and Beren SANDERs, The spectrum of the equivariant stable homotopy category of a finite group, Invent. Math. 208 (2017), 283-326. MR 3621837

[3] ANDREAS DRESS, A characterisation of solvable groups, Math. Z. 110 (1969), 213-217. MR 0248239

[4] Michael J. Hopkins and JefFreY H. SMith, Nilpotence and stable homotopy theory, II, Ann. of Math. (2) 148 (1998), 1-49. MR 1652975

Image Credits

Figures 1 and 2 courtesy of Paul Balmer.

Photo of Paul Balmer courtesy of P. Croset.
Paul Balmer

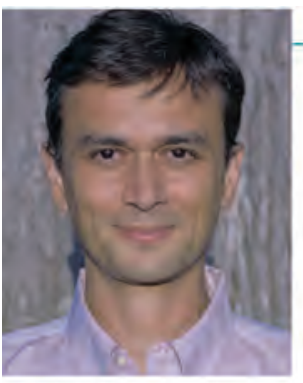

\section{ABOUT THEAUTHOR}

Paul Balmer is working on the development of "tensor-triangular geometry," an umbrella topic that touches parts of algebraic geometry, stable homotopy theory, and modular representation theory.

\section{Double Affine Hecke Algebras and Their Applications}

\section{by Pavel Etingof}

I am very excited to have been asked to deliver an invited address at the Fall 2017 meeting of the AMS Western Section (UC Riverside). I will talk about double affine Hecke algebras and their applications.

It is well known that studying the representation theory of various algebraic structures is a rich source for unifying a number of parts of mathematics. Double affine Hecke algebras are a particular type of algebraic structure whose representation theory has recently become quite important. They were discovered twenty-five years ago by I. Cherednik as a tool for proving the Macdonald conjectures about orthogonal polynomials attached to root systems. But since then it has become clear that they have a much broader meaning. Nowadays they not only play a central role in representation theory but also have numerous connections to many other fields-integrable systems, quantum groups, knot theory, algebraic geometry, and combinatorics, for example.

Double affine Hecke algebras are often defined by a long list of relations, but these relations actually have a simple meaning in terms of elementary topology. Namely, let $\Sigma$ be a connected orientable 2-dimensional surface, and let $C_{n}(\Sigma)$ be the configuration space of $n$-tuples of distinct unlabelled points on $\Sigma$, i.e., the complement in $\Sigma^{n} / S_{n}$ of the loci where some of the points coincide. The fundamental group $B_{n}(\Sigma):=\pi_{1}\left(C_{n}(\Sigma)\right)$ is called the braid group of $\Sigma$. This group contains an element $T$ that corresponds to two of the points exchanging their positions. More precisely, since we haven't fixed a base point, this element is defined only up to conjugation. However, the quotient group $B_{n}(\Sigma) /\left(T^{2}\right)$ of $B_{n}(\Sigma)$ by the relation $T^{2}=1$ is welldefined. By the Seifert-van Kampen theorem, quotienting by this relation corresponds to gluing back into $C_{n}(\Sigma)$ the loci where some points coincide, which gives the orbifold $\Sigma^{n} / S_{n}$. Thus, $B_{n}(\Sigma) /\left(T^{2}\right)$ is the orbifold fundamental group of this orbifold; i.e., it is isomorphic to $S_{n} \ltimes \pi_{1}(\Sigma)^{n}$.

Pavel Etingof is professor of mathematics at MIT. His e-mail address is etingof@math.mit.edu.

For permission to reprint this article, please contact: reprint-permission@ams . org.

DOI: http://dx.doi.org/10.1090/noti1592 


\section{AMS FALL WESTERN SECTIONAL SAMPLER}

Now let us pass from groups to group algebras over some commutative base ring $k$ (for example, $k=\mathbb{C}$ ). Then the relation $T^{2}=1$ may be rewritten as $T^{2}-1=0$ or $(T-$ $1)(T+1)=0$. Thus, given $t_{1}, t_{2} \in k$ (usually assumed to be invertible), we may consider a deformation of this relation:

$$
\left(T-t_{1}\right)\left(T+t_{2}\right)=0 .
$$

Definition 1. The Hecke algebra of $\Sigma, H_{t_{1}, t_{2}}(\Sigma)$ is the quotient of the group algebra $k\left[B_{n}(\Sigma)\right]$ by relation (1).

One can show that unless $\Sigma=S^{2}$ is the 2-sphere, this gives a flat deformation of $k\left[S_{n} \ltimes \pi_{1}(\Sigma)^{n}\right]$ (in the formal sense).

For example, when $\Sigma$ is the 2-plane then $H_{t_{1}, t_{2}}(\Sigma)$ is the finite Hecke algebra, and when $\Sigma$ is the cylinder $H_{t_{1}, t_{2}}(\Sigma)$ is the affine Hecke algebra (in these cases, the algebra actually depends only on $\left.t:=t_{1} / t_{2}\right)$. This motivates the following definition.

Definition 2. The double affine Hecke algebra is the algebra $H_{t_{1}, t_{2}}(\mathbf{T})$, where $\mathbf{T}$ is the 2-torus.

Here the word "double" refers to the fact that the torus has two independent 1-cycles.

The algebra $H_{t_{1}, t_{2}}(\mathbf{T})$ is thus a flat deformation of $k\left[S_{n} \ltimes \mathbb{Z}^{2 n}\right]$, and, unlike the previous two examples, it now genuinely depends on both parameters $t_{1}, t_{2}$.

In a similar way one can define the double affine Hecke algebra of a finite crystallographic reflection group (i.e., Weyl group) $W$ with reflection representation $V$. If $W=S_{n}$ and $V=\mathbb{C}^{n}$, this recovers the algebra $H_{t_{1}, t_{2}}(\mathbf{T})$ defined above.

In my talk, I will discuss the basic properties of double affine Hecke algebras and touch upon some applications.

\section{ABOUT THE AUTHOR}

Pavel Etingof's research focuses on double affine Hecke algebras, quantum groups, and tensor categories. He also runs PRIMES, a research program in mathematics, engineering, and science at MIT.

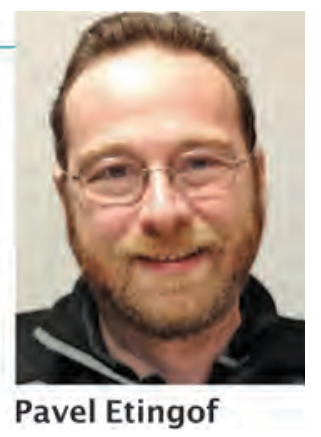

Combinatorics, Categorification, and Crystals by Monica Vazirani

Monica Vazirani is professor of mathematics at the University of California at Davis. Her e-mail address is mjvazirani Qucdavis.edu.

For permission to reprint this article, please contact: reprint-permission@ams.org.

DOI: http://dx.doi.org/10.1090/noti1593
ABSTRACT. Categorification attempts to replace sets or algebraic and geometric structures with more general categories. It has enjoyed amazing successes, such as Khovanov homology categorifying the Jones polynomial knot invariant, KLR algebras categorifying quantum groups, or SoergeI bimodules categorifying Hecke algebras. Many of the algebras we see in categorification can be described diagrammatically. This is related to a historic motivation for categorification: to construct knot and link invariants. The payoffs to finding these richer structures include not only constructing finer knot invariants, but proving positivity results and producing some fantastic mathematics.

In this article, we will focus on the second example, that is, on quantum groups as well as their crystal bases or canonical bases, which exhibit the positivity and integrality that is a trademark feature of a decategorified structure.

In combinatorics, we love to see phenomena that exhibit integrality and positivity. It means there is something interesting to count. It is special for positive integers to occur as structure constants or change of basis coefficients or the coefficients of a polynomial or power series. When this happens it is a bit of an art form to discover a combinatorial structure that realizes these numbers. Once discovered, there can be unexpected payoffs. Further, it can turn computing such coefficients into a fun game, such as counting all the ways to rearrange tiles of a given shape on a board.

The program of categorification is another art form that has enjoyed amazing successes. It is an art form because the process of categorification is not at all well defined. Just as in combinatorics we seek to replace a positive integer with an interesting set of that cardinality, in categorification sets and functions should be replaced with categories and functors. But the process need not start with the category $\mathfrak{S e t s}$, and it is easier to provide a precise definition of the reverse notion, called "decategorification." In the classical setting, decategorification is a well-defined process that turns isomorphic objects of a category into objects that are equal. In a more general setting, decategorification includes passing from a category $\mathfrak{C}$ to its Grothendieck group $G_{0}(\mathfrak{C})$ or its split Grothendieck group $K_{0}(\mathfrak{C})$ or its Trace. In geometric settings, homology theories and cohomology theories are also examples of decategorification. The space that arises from decategorifying a particular category $\mathfrak{C}$ may have interesting algebraic structure that results from the structure of $\mathfrak{C}^{\mathfrak{a}}$. For example, the decategorification may be an abelian group, a ring, a Hopf algebra, or the representation space of some other algebra, as in some of the examples above.

Let us first consider the category $\mathfrak{V e c t}_{\mathbb{C}}$ of finitedimensional vector spaces, which we decategorify by taking the Grothendieck group $G_{0}\left(\mathfrak{V e c t}_{\mathbb{C}}\right) . G_{0}\left(\mathfrak{V}_{\mathfrak{e c t}}\right)$ is the $\mathbb{Z}$-module generated by isomorphism classes of vector spaces, with the relation $[B]=[A]+[C]$ whenever $0 \rightarrow A \rightarrow B \rightarrow C \rightarrow 0$ is exact. Hence $G_{0}\left(\mathfrak{V e c t}_{\mathbb{C}}\right) \simeq \mathbb{Z}$. In fact, this is a ring isomorphism, as $\mathfrak{V e c t}_{\mathbb{C}}$ has the operations of direct sum and tensor product, which descend to + and $\times$ in $\mathbb{Z}$. (But we will continue to call $G_{0}(\mathfrak{C})$ the Grothendieck 


\section{AMS FALL WESTERN SECTIONAL SAMPLER}

group even when endowed with other structures.) Hence a categorification of $\mathbb{Z}$ is $\mathfrak{C}=\mathfrak{V}_{\mathfrak{e c t}}$, and in the process we lift the integer 5 to a 5 -dimensional vector space.

Combinatorialists have been categorifying for decades, even if not thinking of it in that way, often taking $\mathfrak{C}$ to be Sets-wonderfully interesting (finite) sets that look like puzzle pieces. Decategorification here sends a set to its isomorphism class, which we can represent by its cardinality, as two sets are isomorphic exactly when they have the same cardinality. Hence it is easy to decategorify and extract the number 5 from $\left\{(4),(31),\left(2^{2}\right),\left(21^{2}\right),\left(1^{4}\right)\right\}$, the set of integer partitions of 4 . But it is not so straightforward to reverse this. Note that 5 can also be realized as the number of standard Young tableaux of shape (32) or the number of ways to triangulate a pentagon or the size of a basis of our favorite 5-dimensional vector space or indeed the number of elements in any set of cardinality 5 .

Next, consider $\mathfrak{C}=\mathbb{C}\left[\mathfrak{S}_{4}\right]$-mod, or, better yet, let $\mathfrak{C}=$ $\bigoplus_{n \in \mathbb{N}} \mathbb{C}\left[\mathfrak{S}_{n}\right]$-mod, which has induction and restriction functors that let us pass between different $\mathbb{N}$-graded pieces. Its Grothendieck group is isomorphic to the ring (or Hopf algebra) of symmetric functions. In other words, we say the symmetric group categorifies symmetric functions. More precisely, $\mathbb{C}\left[\mathfrak{S}_{4}\right]$-mod decategorifies to the space of degree 4 symmetric functions, which we could further decategorify to the set of partitions of 4 . Further, the spaces have distinguished bases: the 5 simple $\mathbb{C}\left[\mathfrak{S}_{4}\right]$ modules correspond to the 5 Schur functions of degree 4 , which are both naturally indexed by the 5 partitions of 4 .

Additionally, refined induction and restriction functors decategorify to linear operators that give the graded vector space $\bigoplus_{n \in \mathbb{N}} \mathbb{C} \otimes_{\mathbb{Z}} G_{0}\left(\mathbb{C}\left[\mathfrak{S}_{n}\right]\right.$-mod) the structure of a highest weight representation $V\left(\Lambda_{0}\right)$ of the Lie algebra $\mathfrak{s l}_{\infty}$ or of its enveloping algebra $U\left(\mathfrak{s l}_{\infty}\right)$. In other words, symmetric groups also categorify $V\left(\Lambda_{0}\right)$, sometimes known as Fock space. The isomorphism classes of simple modules form a distinguished (weight) basis of $V\left(\Lambda_{0}\right)$. As multiplicities of simple modules in a composition series are nonnegative integers, this basis enjoys wonderful positivity properties. It is a crystal basis, a dual Lusztig canonical basis, and a Kashiwara upper global crystal basis. (Dually, one can work with indecomposable projectives for the split Grothendieck group $K_{0}(\mathfrak{C})$.) We can even replace $\mathbb{C}$ with $\mathbb{F}_{p}$, and $\mathfrak{s l}_{\infty}$ with $\hat{\mathfrak{s l}}_{p}$, and then the simples yield a dual $p$-canonical basis, which has even stronger positivity properties than the dual canonical basis in this case. The known integrality and positivity properties of crystal bases and canonical bases as well as their combinatorial structure were a hint that $V\left(\Lambda_{0}\right)$ could be categorified. We understood that Young's lattice of partitions described the combinatorics of the symmetric group $\left(\mathbb{C}\left[\mathfrak{S}_{n}\right]\right.$-mod) as well as that of $V\left(\Lambda_{0}\right)$ before we knew why.

The above story raises the question of whether we can replace $U\left(\mathfrak{s l}_{\infty}\right)$ with the quantum Kac-Moody algebra $U_{q}(\mathfrak{g})$ of type $X$, as this is also a setting in which we have crystal bases and canonical bases. In groundbreaking work Khovanov-Lauda and Rouquier construct $\mathfrak{C}$ as the category of modules over the KLR algebra, or quiver Hecke algebra, of arbitrary symmetrizable type $X$. I have heard Kleshchev describe KLR algebras as being type $A$ (like the symmetric group $\mathfrak{S}_{n}$ ) but in characteristic $X$. For example, characteristic $\hat{\mathfrak{s l}}_{p}$ behaves like the more familiar characteristic $p$, but characteristic $E_{8}$ also makes sense. I love this description.

The KLR algebra is graded, which introduces the quantum parameter $q$. We replace $n \in \mathbb{N}$ with $v \in Q^{+}$, the positive cone in the root lattice; replace $\mathfrak{S}_{n}$ with the graded algebra $R(v)$; and replace $\bigoplus_{n \in \mathbb{N}} \mathbb{C}\left[\mathfrak{S}_{n}\right]$ with $R=\bigoplus_{v \in Q^{+}} R(v)$. Then $\mathfrak{C}=R$-mod categorifies $U_{q}^{+}(\mathfrak{g})$, and certain quotients called cyclotomic KLR algebras categorify highest weight representations $V(\lambda)$ for dominant integral weights $\lambda \in P^{+}$. Then we recover the crystal graph $B(\lambda)$ via the simple $R$-modules.

The crystal graph $B(\lambda)$ is a combinatorial skeleton of the irreducible highest weight representation $V(\lambda)$ of $U_{q}(\mathfrak{g})$. In "the $q \rightarrow 0$ limit" $V(\lambda)$ has a crystal basis that comprises the nodes of the graph $B(\lambda)$. The graph has other data, including directed colored edges that are roughly limits of an integral form of raising operators.

Over $\mathfrak{g}=\mathfrak{s l}_{\infty}$ the crystal graph $B\left(\Lambda_{0}\right)$ looks like Young's lattice of partitions with extra decoration. Although the $\mu$-weight space of $V(\lambda)$ can have high multiplicity, a node of $B(\lambda)$ can be a source (or sink) of at most one edge of given color. In this way, the crystal knows how to "break ties" and fits into a multiplicity-free setting, much like the branching rule for the symmetric group over $\mathbb{C}$ is multiplicity-free. Crystal graphs are one of my favorite combinatorial tools. I like to believe that in hindsight the blueprints to the KLR algebras were dictated by the crystal graphs. Their generators and relations are precisely what they need to be for restriction functors to have the right multiplicity-free properties and to yield the appropriate subcrystals in rank 2 . Beautiful combinatorics can be harvested from this program of categorification, but (to mix a few metaphors) I also think we glimpse combinatorial shadows before we know what is casting them, and we should keep an eye out for these blueprints.

\section{Photo Credit}

Photo of Monica Vazirani courtesy of Dorothy Buck.

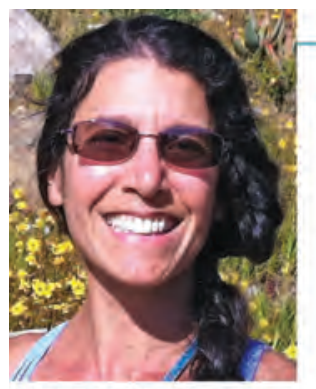

ABOUT THE AUTHOR

Monica Vazirani's research is in combinatorial representation theory. 


\section{Discrete Differential Geometry}

Linking Analytical Aspects

of Shape with Computation

\section{AMS Short Course}

\section{January 8-9, 2018, San Diego}

(in conjunction with the Joint Mathematics Meetings)

The American Mathematical Society's Short Courses provide entrants with rich introductions to emergent areas of applied mathematics - fuel for participants' curiosity, discovery, or research.

In 2018 the Short Course lecturers focus on "mimetic discretization"-key properties of continuous systems are preserved in discrete approximations - and on appli-

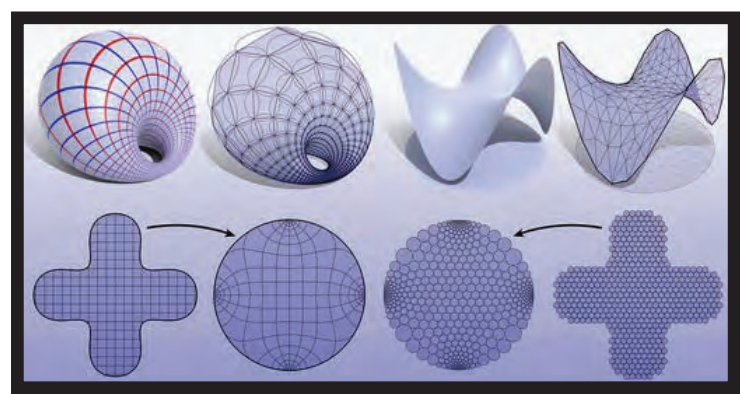
cations in biology, mechanics, design, and architecture.

\section{Course Organizer}

Keenan Crane, Carnegie Mellon University

Lecture Topics

Discrete Laplace Operators, Max Wardetzky, University of Göttingen

Discrete Parametric Surfaces, Johannes Wallner, Technische Universität Graz

Discrete Mappings, Yaron Lipman, Weizmann Institute

Discrete Conformal Geometry, Keenan Crane, Carnegie Mellon University

Optimal Transportation on Discrete Domains, Justin Solomon, Massachusetts Institute of Technology

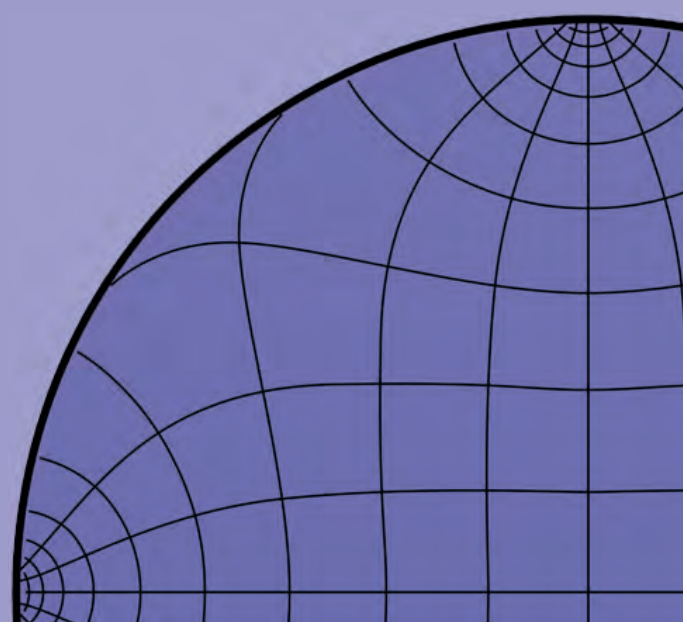

Learn more and register: www.ams.org/meetings/short-courses/ short-course-general 


\section{The Concept of Varifold}

\section{Ulrich Menne}

ABSTRACT. We survey-by means of 20 examplesthe concept of varifold, as generalised submanifold, with emphasis on regularity of integral varifolds with mean curvature, while keeping prerequisites to a minimum. Integral varifolds are the natural language for studying the variational theory of the area integrand if one considers, for instance, existence or regularity of stationary (or stable) surfaces of dimension at least three or the limiting behaviour of sequences of smooth submanifolds under area and mean curvature bounds.

\section{Introduction}

\section{Motivation}

Apart from generalisation, there are two main reasons to include nonsmooth surfaces in geometric variational problems in Euclidean space or Riemannian manifolds: firstly, the separation of existence proofs from regularity considerations and, secondly, the modelling of nonsmooth physical objects. Following the first principle, varifolds were introduced by F. Almgren in 1965 to prove, for every intermediate dimension, the existence of a generalised minimal surface (i.e., a surface with vanishing first variation of area) in a given compact smooth Riemannian manifold. Then, in 1972, an important partial regularity result for such varifolds was established by W. Allard in his foundational paper "On the first variation of a varifold" [2]. These pioneering works still have a strong influence in geometric analysis as well as related fields. Particularly prominent examples are the proof of the Willmore conjecture by F. Marques and A. Neves and the development of a regularity theory for stable generalised minimal surfaces in one codimension by N. Wickramasekera, both published in 2014. Following the second principle, varifolds were employed by Almgren's PhD student K. Brakke in 1978 to create a mathematical model of the motion

Ulrich Menne currently holds an interim professorship at the University of Leipzig and a postdoctoral position at the Max Planck Institute for Mathematics in the Sciences. His e-mail address is U1rich. Menne@math.uni-1eipzig.de.

For permission to reprint this article, please contact: reprint-permission@ams.org.

DOI: http://dx.doi.org/10.1090/noti1589 of grain boundaries in an annealing pure metal. This was the starting point of the rapid development of mean curvature flow, even for smooth surfaces.

\section{What is a Varifold?}

Constructing nonparametric models of nonsmooth surfaces usually requires entering the realm of geometric measure theory. In the simplest case, we associate to each smooth surface $M$ the measure over the ambient space whose value at a set $A$ equals the surface measure of the intersection $A \cap M$. For varifolds, it is in fact expedient to similarly record information on the tangent planes of the surfaces. This yields weak continuity of area, basic compactness theorems, and the possibility to retain tangential information in the limit.

\section{Main Topics Covered}

We will introduce the notational infrastructure together

the attempt to
comprise all
objects that
should be
considered
m-dimensional
surfaces
with a variety of examples. This will then allow us to formulate the compactness theorem for integral varifolds, Theorem 1, and two key regularity theorems for integral varifolds with mean curvature, Theorems 2 and 3 . The accompanying drawings generally stress certain aspects deemed important at the expense of accuracy. A version of this article with additional references is available from https://arxiv.org/abs/1705.05253.

\section{Notation}

Suppose throughout this note that $m$ and $n$ are integers and $1 \leq m \leq n$. We will make use of the $m$-dimensional Hausdorff measure $\mathcal{H}^{m}$ over $\mathbf{R}^{n}$. This measure is one of several natural outer measures over $\mathbf{R}^{n}$ which associate the usual values with subsets of $m$-dimensional continuously differentiable submanifolds of $\mathbf{R}^{n}$. 


\section{First Order Quantities}

Amongst the initial reasons for developing the notion of $m$ dimensional varifold in $\mathbf{R}^{n}$ was the attempt to comprise all objects that should be considered $m$-dimensional surfaces of locally finite area in $\mathbf{R}^{n}$. Its definition is tailored for compactness.

Definition (Varifold and weight). If $\mathbf{G}(m, n)$ is the space of unoriented $m$-dimensional subspaces of $\mathbf{R}^{n}$, endowed with its natural topology, then by an $m$-dimensional varifold $V$ in $\mathbf{R}^{n}$, we mean a Radon measure $V$ over $\mathbf{R}^{n} \times$ $\mathbf{G}(n, m)$, and we denote by $\|V\|$ the weight of $V$, that is, its canonical projection into $\mathbf{R}^{n}$.

The theory of Radon measures yields a metrisable topology on the space of $m$-dimensional varifolds in $\mathbf{R}^{n}$ such that $V_{i} \rightarrow V$ as $i \rightarrow \infty$ if and only if

$$
\int k \mathrm{~d} V_{i} \rightarrow \int k \mathrm{~d} V \text { as } i \rightarrow \infty
$$

whenever $k: \mathbf{R}^{n} \times \mathbf{G}(m, n) \rightarrow \mathbf{R}$ is a continuous function with compact support.

Proposition (Basic compactness). Whenever $\kappa$ is a realvalued function on the bounded open subsets of $\mathbf{R}^{n}$, the set of m-dimensional varifolds $V$ in $\mathbf{R}^{n}$ satisfying $\|V\|(Z) \leq$ $\kappa(Z)$ for each bounded open subset $Z$ of $\mathbf{R}^{n}$ is compact.

In general, neither does the weight $\|V\|$ of $V$ need to be $m$-dimensional, nor does the Grassmannian information of $V$ need to be related to the geometry of $\|V\|$ :

Example 1 (Region with associated plane). For each plane $T \in \mathbf{G}(n, m)$ and each open subset $Z$ of $\mathbf{R}^{n}$, the product of the Lebesgue measure restricted to $Z$, with the Dirac measure concentrated at $T$, is an $m$-dimensional varifold in $\mathbf{R}^{n}$.

Example 2 (Point with associated plane). For $a \in \mathbf{R}^{n}$ and $T \in \mathbf{G}(n, m)$, the Dirac measure concentrated at $(a, T)$ is an $m$-dimensional varifold in $\mathbf{R}^{n}$.

This generality is in fact expedient to describe higher(or lower-) dimensional approximations of more regular $m$ dimensional surfaces, so as to include information on the $m$-dimensional tangent planes of the limit. Such situations may be realised by elaborating on Examples 1 and 2 . However, we will mainly consider integral varifolds, that is, varifolds in the spirit of the next basic example, which avoid the peculiarities of the preceding two examples.

Example 3 (Part of a submanifold). If $B$ is a Borel subset of a closed $m$-dimensional continuously differentiable submanifold $M$ of $\mathbf{R}^{n}$, then, by Riesz's representation theorem, the associated varifold $\mathbf{v}_{m}(B)$ in $\mathbf{R}^{n}$ may be defined by

$$
\int k \mathrm{~d} \mathbf{v}_{m}(B)=\int_{B} k(x, \operatorname{Tan}(M, x)) \mathrm{d} \mathcal{H}^{m} x
$$

whenever $k: \mathbf{R}^{n} \times \mathbf{G}(m, n) \rightarrow \mathbf{R}$ is a continuous function with compact support, where $\operatorname{Tan}(M, x)$ is the tangent space of $M$ at $x$. Then, the weight $\left\|\mathbf{v}_{m}(B)\right\|$ over $\mathbf{R}^{n}$ equals the restriction $\mathcal{H}^{m}\llcorner B$ of $m$-dimensional Hausdorff measure to $B$.
Definition (Integral varifold). An $m$-dimensional varifold $V$ in $\mathbf{R}^{n}$ is called integral if and only if there exists a sequence of Borel subsets $B_{i}$ of closed $m$-dimensional continuously differentiable submanifolds $M_{i}$ of $\mathbf{R}^{n}$ with $V=\sum_{i=1}^{\infty} \mathbf{v}_{m}\left(B_{i}\right)$.

An integral varifold $V$ is determined by its weight $\|V\|$; in fact, associated to $\|V\|$ there are a multiplicity function $\Theta$ and a tangent plane function $\tau$ with values in the nonnegative integers and $\mathbf{G}(n, m)$, respectively, such that

$$
V(k)=\int k(x, \tau(x)) \Theta(x) \mathrm{d} \mathcal{H}^{m} x
$$

whenever $k: \mathbf{R}^{n} \times \mathbf{G}(m, n) \rightarrow \mathbf{R}$ is a continuous function with compact support.

Example 4 (Sum of two submanifolds). Suppose $M_{i}$ are closed $m$-dimensional continuously differentiable submanifolds $M_{i}$ of $\mathbf{R}^{n}$, for $i \in\{1,2\}$, and $V=\mathbf{v}_{m}\left(M_{1}\right)+$ $\mathbf{v}_{m}\left(M_{2}\right)$. Then, for $\mathcal{H}^{m}$ almost all $z \in M_{1} \cap M_{2}$, we have

$$
\Theta(z)=2 \text { and } \tau(z)=\operatorname{Tan}\left(M_{1}, z\right)=\operatorname{Tan}\left(M_{2}, z\right) .
$$

The theory of varifolds is particularly successful in the variational study of the area integrand. To describe some aspects of this, diffeomorphic deformations are needed in order to define and compute its first variation.

Definition (Area of a varifold). If $B$ is a Borel subset of $\mathbf{R}^{n}$ and $V$ is called an $m$-dimensional varifold $V$ in $\mathbf{R}^{n}$, then the area of $V$ in $B$ equals $\|V\|(B)$.

Example 5 (Area via multiplicity). If $V$ is integral, then $\|V\|(B)=\int_{B} \Theta \mathrm{d} \mathcal{H}^{m}$.

Definition (Push-forward). Suppose $V$ is an $m$-dimensional varifold in $\mathbf{R}^{n}$ and $\phi$ is a continuously differentiable diffeomorphism of $\mathbf{R}^{n}$ onto $\mathbf{R}^{n}$. Then, the push-forward of $V$ by $\phi$ is the $m$-dimensional varifold $\phi_{\#} V$ defined by

$\int k \mathrm{~d} \phi_{\#} V=\int k(\phi(z)$, image $(\mathrm{D} \phi(z) \mid S)) j_{m} \phi(z, S) \mathrm{d} V(z, S)$ whenever $k: \mathbf{R}^{n} \times \mathbf{G}(m, n) \rightarrow \mathbf{R}$ is a continuous function with compact support. Here, $j_{m} \phi(z, S)$ denotes the $m$-dimensional Jacobian of the restriction to $S$ of the differential D $\phi(z): \mathbf{R}^{n} \rightarrow \mathbf{R}^{n}$.

Example 6 (Push-forward). If $B$ and $M$ are as in Example 3, then, by the transformation formula (or area formula), $\phi_{\#}\left(\mathbf{v}_{m}(B)\right)=\mathbf{v}_{m}(\operatorname{image}(\phi \mid B))$.

Definition (First variation). The first variation of a varifold $V$ in $\mathbf{R}^{n}$ is defined by

$$
(\delta V)(\theta)=\int \operatorname{trace}\left(\mathrm{D} \theta(z) \circ S_{\natural}\right) \mathrm{d} V(z, S)
$$

whenever $\theta: \mathbf{R}^{n} \rightarrow \mathbf{R}^{n}$ is a smooth vector field with compact support, where $S_{\natural}$ denotes the canonical orthogonal projection of $\mathbf{R}^{n}$ onto $S$.

Example 7 (Meaning of the first variation). If the maps $\phi_{t}: \mathbf{R}^{n} \rightarrow \mathbf{R}^{n}$ satisfy $\phi_{t}(z)=z+t \theta(z)$ whenever $t \in$ $\mathbf{R},|t| \operatorname{Lip} \theta<1$, and $z \in \mathbf{R}^{n}$, then one may verify that, for such $t, \phi_{t}$ is a smooth diffeomorphism of $\mathbf{R}^{n}$ onto $\mathbf{R}^{n}$ and $\left\|\left(\phi_{t}\right)_{\#} V\right\|(\operatorname{spt} \theta)$ is a smooth function of $t$ whose differential at 0 equals $(\delta V)(\theta)$. 
Example 8 (First variation of a smooth submanifold I). Suppose that $M$ is a properly embedded, twice continuously differentiable $m$-dimensional submanifold-withboundary in $\mathbf{R}^{n}$ and $V=\mathbf{v}_{m}(M)$. Then, integrating by parts, one obtains

$$
\begin{aligned}
(\delta V)(\theta)= & -\int_{M} \mathbf{h}(M, z) \bullet \theta(z) \mathrm{d} \mathcal{H}^{m} z \\
& +\int_{\partial M} v(M, z) \bullet \theta(z) \mathrm{d} \mathcal{H}^{m-1} z,
\end{aligned}
$$

where $\mathbf{h}(M, z) \in \mathbf{R}^{n}$ is the mean curvature vector of $M$ at $z \in M, v(M, z)$ is the outer normal of $M$ at $z \in \partial M$, and $\bullet$ denotes the inner product in $\mathbf{R}^{n}$. In fact, $\mathbf{h}(M, z)$ belongs to the normal space, $\operatorname{Nor}(M, z)$, of $M$ at $z$.

\section{Second Order Quantities}

In general, even integral varifolds-which already are significantly more regular than general varifolds-give meaning only to first order properties such as tangent planes but do not possess any second order properties such as curvaFor varifolds of tures. Yet, for varifolds of locally bounded tion, one may define mean first variation, one may define mean curvature.

Example 9 (A particular continuously differentiable submanifold without-even approximate-curvatures; see R. Kohn [3]). There exists a nonempty closed continuously differentiable $m$-dimensional submanifold $M$ of $\mathbf{R}^{m+1}$ such that for any twice continuously differentiable $m$-dimensional submanifold $N$ of $\mathbf{R}^{m+1}$, there holds $\mathcal{H}^{m}(M \cap N)=0$.

Definition (Locally bounded first variation). A varifold $V$ in $\mathbf{R}^{n}$ is said to be of locally bounded first variation if and only if $\|\delta V\|(Z)$, defined as

$$
\begin{gathered}
\sup \{(\delta V)(\theta): \theta \text { a smooth vector field compactly } \\
\text { supported in } Z \text { with }|\theta| \leq 1\}
\end{gathered}
$$

whenever $Z$ is an open subset of $\mathbf{R}^{n}$, is finite on bounded sets. Then $\|\delta V\|$ may be uniquely extended to a Radon measure over $\mathbf{R}^{n}$, also denoted by $\|\delta V\|$.

Clearly, for any open subset $Z$ of $\mathbf{R}^{n},\|\delta V\|(Z)$ is lower semicontinuous in $V$.

Example 10 (Functions of locally bounded variation). If $m=n$, then, $m$-dimensional varifolds in $\mathbf{R}^{m}$ of locally bounded first variation are in natural correspondence to real-valued functions of locally bounded first variation on $\mathbf{R}^{m}$.

Example 11 (First variation of a smooth submanifold II). If $M$ and $V$ are as in Example 8, then we have $\|\delta V\|=$ $|\mathbf{h}(M, \cdot)| \mathcal{H}^{m}\left\llcorner M+\mathcal{H}^{m-1}\llcorner\partial M\right.$.

Theorem 1 (Compactness of integral varifolds; see Allard $[2,6.4])$. If $\kappa$ is a real-valued function on the bounded open subsets of $\mathbf{R}^{n}$, then the set of integral varifolds with $\|V\|(Z)+\|\delta V\|(Z) \leq \kappa(Z)$ for each bounded open subset $Z$ of $\mathbf{R}^{n}$ is compact.
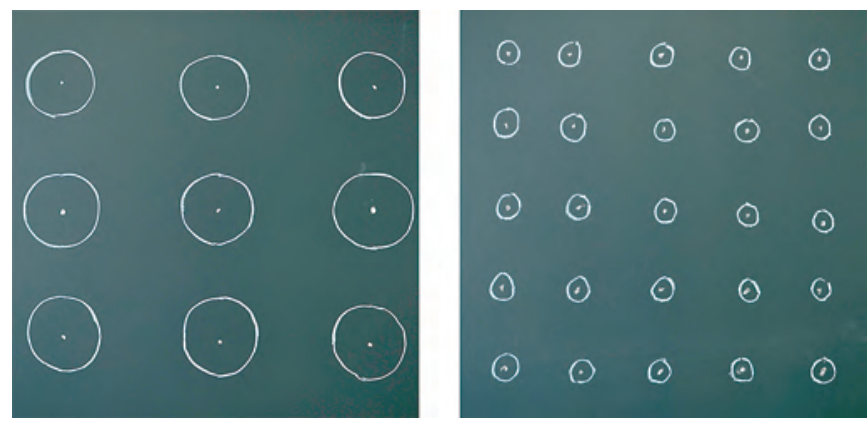

Figure 1. One-dimensional submanifolds (by the spheres drawn) of $\mathrm{R}^{2}$ converging to a limit nonzero, nonintegral varifold with all one-dimensional tangent planes equally weighted at every point of $R^{2}$.

The preceding theorem is fundamental both for varifolds and the consideration of limits of submanifolds. The summand $\|\delta V\|(Z)$ therein may not be omitted:

Example 12 (Lattices of spheres; see Figure 1). If $V_{i}$ is the varifold associated to

$M_{i}=\mathbf{R}^{m+1} \cap\left\{z:|i z-a|=3^{-1} i^{-1 / m}\right.$ for some $\left.a \in \mathbf{Z}^{m+1}\right\}$, for every positive integer $i$, then the nonzero limit of this sequence is the product of the Lebesgue measure over $\mathbf{R}^{m+1}$ with an invariant Radon over $\mathbf{G}(m+1, m)$.

Definition (Mean curvature). Suppose $V$ is a varifold in $\mathbf{R}^{n}$ of locally bounded first variation and $\sigma$ is the singular part of $\|\delta V\|$ with respect to $\|V\|$. Then, there exist a $\sigma$ almost unique, $\sigma$ measurable function $\boldsymbol{\eta}(V, \cdot)$ with values in the unit sphere $\mathrm{S}^{n-1}$ as well as a $\|V\|$ almost unique, $\|V\|$ measurable, locally $\|V\|$ summable, $\mathbf{R}^{n}$-valued function $\mathbf{h}(V, \cdot)$-called the mean curvature of $V$-satisfying

$$
\begin{aligned}
(\delta V)(\theta)= & -\int \mathbf{h}(V, z) \bullet \theta(z) \mathrm{d}\|V\| z \\
& +\int \boldsymbol{\eta}(V, z) \bullet \theta(z) \mathrm{d} \sigma z
\end{aligned}
$$

whenever $\theta: \mathbf{R}^{n} \rightarrow \mathbf{R}^{n}$ is a smooth vector field with compact support.

Example 13 (Varifold mean curvature of a smooth submanifold). If $M, V$, and $v$ are as in Example 8, then $\sigma=$ $\mathcal{H}^{m-1}\llcorner\partial M, \boldsymbol{\eta}(V, z)=v(M, z)$ for $\sigma$ almost all $z$, and $\mathbf{h}(V, z)=\mathbf{h}(M, z)$ for $\|V\|$ almost all $z$.

Yet, in general, the mean curvature vector may have tangential parts, and the behaviour of $\sigma$ and $\boldsymbol{\eta}\left(V, \cdot^{-}\right)$may differ from that of boundary and outer normal:

Example 14 (Weighted plane). If $m=n, \Theta$ is a positive, continuously differentiable function on $\mathbf{R}^{m}$, and $V(k)=$ $\int k\left(z, \mathbf{R}^{m}\right) \Theta(z) \mathrm{d} \mathcal{H}^{m} z$ whenever $k: \mathbf{R}^{m} \times \mathbf{G}(m, m) \rightarrow \mathbf{R}$ is a continuous function with compact support, then

$$
\mathbf{h}(V, z)=\operatorname{grad}(\log \circ \Theta)(z) \text { for }\|V\| \text { almost all } z \text {. }
$$

Example 15 (Primitive of the Cantor function). If $C$ is the Cantor set in $\mathbf{R}, f: \mathbf{R} \rightarrow \mathbf{R}$ is the associated function (i.e., $f(x)=\mathcal{H}^{d}(C \cap\{x: \chi \leq x\})$ for $x \in \mathbf{R}$, where $d=\log 2(\log 3)$, and $V$ is the varifold in $\mathbf{R}^{2} \simeq \mathbf{R} \times \mathbf{R}$ associated to the graph of a primitive function of $f$, then $V$ 

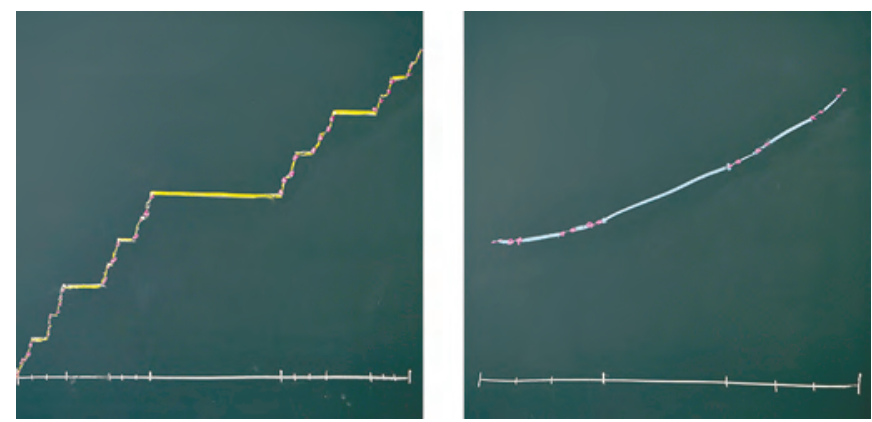

Figure 2. Varifold (blue, on the right) with fractal "boundary." Left: Cantor function. Right: A primitive of the Cantor function.

is an integral varifold of locally bounded first variation, $\mathbf{h}(V, z)=0$ for $\|V\|$ almost all $z$, and spt $\sigma$ corresponds to $C$ via the orthogonal projection onto the domain of $f$, as in Figure 2.

Definition (Classes $H(p)$ of summability of mean curvature). For $1 \leq p \leq \infty$, the class $H(p)$ is defined to consist of all $m$-dimensional varifolds $V$ in $\mathbf{R}^{n}$ of locally bounded first variation satisfying the following three conditions: if $p>1$, then $\|\delta V\|$ is absolutely continuous with respect to $\|V\|$; if $1<p<\infty$, then their mean curvature is locally $p$-th power $\|V\|$ summable; and if $p=\infty$, it is locally $\|V\|$ essentially bounded.

Observe that $\left(\int_{Z}|\mathbf{h}(V, z)|^{p} \mathrm{~d}\|V\| Z\right)^{1 / p}$ is lower semicontinuous in $V \in H(p)$ if $Z$ is an open subset of $\mathbf{R}^{n}$ and $1<p<\infty$; a similar statement holds for $p=\infty$. By Theorem 1 , this entails further compactness theorems for integral varifolds.

Example 16 (Critical scaling for $p=m$ ). If $V \in H(m)$, $0<r<\infty$, and $\phi: \mathbf{R}^{n} \rightarrow \mathbf{R}^{n}$ satisfies $\phi(z)=r z$ for $z \in \mathbf{R}^{n}$, then $\phi_{\#} V \in H(m)$ and we have

$\int_{\text {image }(\phi \mid B)}\left|\mathbf{h}\left(\phi_{\#} V, z\right)\right|^{m} \mathrm{~d}\left\|\phi_{\#} V\right\| z=\int_{B}|\mathbf{h}(V, z)|^{m} \mathrm{~d}\|V\| z$, and, in case $m=1$, additionally $\left\|\delta\left(\phi_{\#} V\right)\right\|(\operatorname{image}(\phi \mid B))=$ $\|\delta V\|(B)$ whenever $B$ is a Borel subset of $\mathbf{R}^{n}$.

\section{Regularity by First Variation Bounds}

For twice continuously differentiable submanifolds $M$, the mean curvature vector $\mathbf{h}(M, z)$ at $z \in M$ is defined as trace of the second fundamental form

$$
\mathbf{b}(M, z): \operatorname{Tan}(M, z) \times \operatorname{Tan}(M, z) \rightarrow \operatorname{Nor}(M, z) .
$$

For varifolds, bounds on the mean curvature (or, more generally, on the first variation) are defined without reference to a second order structure and entail more regularity, similar to the case of weak solutions of the Poisson equation. The key additional challenges are nongraphical behaviour and higher multiplicity:

Example 17 (Cloud of spheres). If $1 \leq p<m<n$ and $Z$ is an open subset of $\mathbf{R}^{n}$, then there exists a countable collection $C$ of $m$-dimensional spheres in $\mathbf{R}^{n}$ such that $V=\sum_{M \in C} \mathbf{V}_{m}(C) \in H(p)$ and spt $\|V\|=$ Closure $Z$.
In contrast, if $p \geq m$, then $\mathcal{H}^{m}\llcorner\operatorname{spt}\|V\| \leq\|V\|$ whenever $V$ is an integral varifold in $H(p)$; in particular, spt $\|V\|$ has locally finite $m$-dimensional measure.

Definition (Singular). An $m$-dimensional varifold $V$ in $\mathbf{R}^{n}$ is called singular at $z$ in spt $\|V\|$ if and only if there is no neighbourhood of $z$ in which $V$ corresponds to a positive multiple of an $m$-dimensional continuously differentiable submanifold.

Example 18 (Zero sets of smooth functions). If $A$ is a closed subset of $\mathbf{R}^{m}$, then there exists a nonnegative smooth function $f: \mathbf{R}^{m} \rightarrow \mathbf{R}$ with $A=\{x: f(x)=0\}$.

Example 19 (Touching; see Figure 3). Suppose $A$ is a closed subset of $\mathbf{R}^{m}$ without interior points such that $\mathcal{H}^{m}(A)>0, f$ is related to $A$ as in Example $18, n=m+1$, $\mathbf{R}^{n} \simeq \mathbf{R}^{m} \times \mathbf{R}$, and $M_{i} \subset \mathbf{R}^{n}$, for $i \in\{1,2\}$, correspond to the graph of $f$, and $\mathbf{R}^{m} \times\{0\}$, respectively. Then, $V=\mathbf{v}_{m}\left(M_{1}\right)+\mathbf{v}_{m}\left(M_{2}\right)$ (see Examples 4 and 13) is an integral varifold in $H(\infty)$ which is singular at each point of $M_{1} \cap M_{2} \simeq A \times\{0\}$.

Proposition (Structure of one-dimensional integral varifolds in $H(1)$; see [4, 12.5]). If $m=1$ and $V \in H(1)$ is an integral varifold, then, near $\|V\|$ almost all points, $V$ may be locally represented by finitely many Lipschitzian graphs.

Proposition (Regularity of one-dimensional integral varifolds with vanishing first variation; see Allard and Almgren [1, §3]). If $m=1$ and $V$ is an integral varifold with $\delta V=0$, then the set of singular points of $V$ has $\mathcal{H}^{m}$ measure zero.

It is a key open problem to determine whether the hypothesis $m=1$ is essential; the difficulty of the case $m \geq 2$ arises from the possible presence of holes:

Example 20 (Swiss cheese; see Brakke or $[4,10.8])$. If $2 \leq m<n$, then there exist $V \in H(\infty)$ integral, $T \in \mathbf{G}(n, m)$, and a Borel subset $B$ of $T \cap \operatorname{spt}\|V\|$ with $\mathcal{H}^{m}(B)>0$ such that no $b \in B$ belongs to the interior of the orthogonal projection of $\mathrm{spt}\|V\|$ onto $T$ relative to $T$. So, for

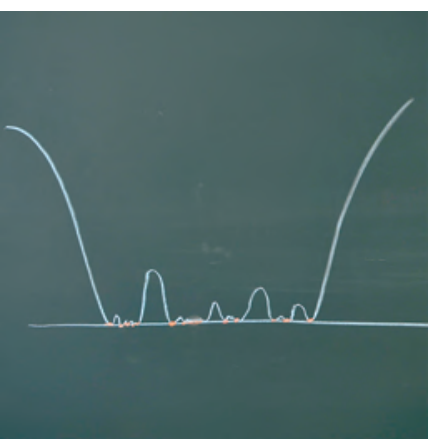

Figure 3. A simple integral varifold with bounded mean curvature whose set of singular points has positive weight measure. $\mathcal{H}^{m}$ almost all $b \in B, \tau(b)=T$ and $V$ is singular at $b$ (cf. Example 4); see Figure 4.

Yet, the preceding varifold needs to have nonsingular points in spt $\|V\|$. To treat $\|V\|$ almost all points, however, weaker concepts of regularity are needed.

Theorem 2 (Allard's regularity theorem-qualitative version; see Allard [2, 8.1(1)]). If $p>m \geq 2$ and $V \in H(p)$ is integral, then the set of singular points of $V$ contains no interior points relative to spt $\|V\|$. 


\section{COMMUNICATION}
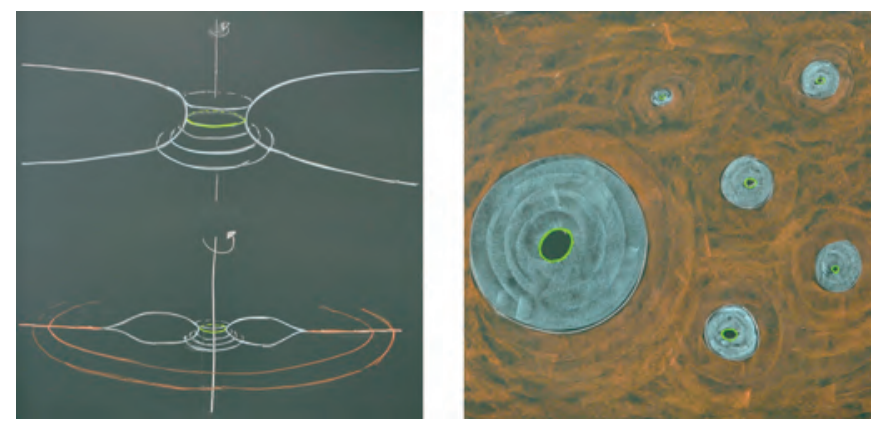

Figure 4. Constructing an integral varifold with bounded mean curvature with holes near a set of positive weight measure. Left: Building blocks (from side). Right: Result (from top).

Theorem 3 (Existence of a second order structure; see [5, 4.8]). If $V$ is an integral varifold in $H(1)$, then there exists a countable collection $C$ of twice continuously differentiable $m$-dimensional submanifolds such that $C$ covers $\|V\|$ almost all of $\mathbf{R}^{n}$. Moreover, for $M \in C$, there holds $\mathbf{h}(V, z)=$ $\mathbf{h}(M, z)$ for $\|V\|$ almost all $z$.

Hence, one may define a concept of second fundamental form of $V$ such that, for $\|V\|$ almost all $z$, its trace is $\mathbf{h}(V, z)$. Moreover, Theorem 3 is the base for studying the fine behaviour of integral varifolds in $H(p)$; see [5] and [4].

\section{References}

[1] W. K. AllaRD and F. J. ALMgRen JR., The structure of stationary one dimensional varifolds with positive density, Invent. Math. 34 (1976), no. 2, pages 83-97. MR 0425741

[2] William K. Allard, On the first variation of a varifold, Ann. of Math. (2) 95 (1972), pages 417-491. MR 0307015

[3] ROBERT V. KOHN, An example concerning approximate differentiation, Indiana Univ. Math. J. 26 (1977), no. 2, pages 393-397. MR 0427559

[4] SŁAwOMIR KolASińSKi and UlRich Menne, Decay rates for the quadratic and super-quadratic tilt-excess of integral varifolds, NoDEA Nonlinear Differential Equations Appl. 24 (2017), Art. 17, pages 1-56. MR 3625810

[5] UlRICH MENNE, Second order rectifiability of integral varifolds of locally bounded first variation, J. Geom. Anal. 23 (2013), no. 2, pages 709-763. MR 3023856

\section{Image Credits}

All figures courtesy of Ulrich Menne.

Author photo courtesy of Max Planck Institute for Gravitational Physics (Albert Einstein Institute).

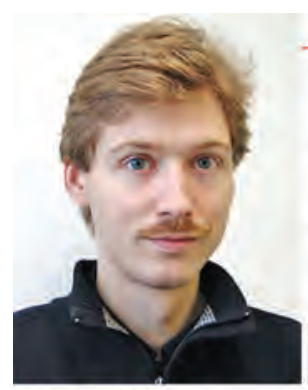

\section{ABOUT THE AUTHOR}

Ulrich Menne's research concerns geometric measure theory, differential and convex geometry, and nonlinear elliptic partial differential equations.

Ulrich Menne 


\title{
A Glimpse into Discrete Differential Geometry
}

\author{
Keenan Crane and Max Wardetzky
}

Communicated by Joel Hass

EDITOR'S NOTE. The organizers of the two-day AMS Short Course on Discrete Differential Geometry have kindly agreed to provide this introduction to the subject. The AMS Short Course runs in conjunction with the 2018 Joint Mathematics Meetings.

The emerging field of discrete differential geometry (DDG) studies discrete analogues of smooth geometric objects, providing an essential link between analytical descriptions and computation. In recent years it has unearthed a rich variety of new perspectives on applied problems in computational anatomy/biology, computational mechanics, industrial design, computational architecture, and digital geometry processing at large. The basic philosophy of discrete differential geometry is that a discrete object like a polyhedron is not merely an approximation of a smooth one, but rather a differential geometric object in its own right. In contrast to traditional numerical analysis which focuses on eliminating approximation error in the limit of refinement (e.g., by taking smaller and smaller finite differences), DDG places an emphasis on the so-called "mimetic" viewpoint, where key properties of a system are preserved exactly, independent of how large or small the elements of a mesh might be. Just as algorithms for simulating mechanical systems might seek to exactly preserve physical invariants such as total energy or momentum, structure-preserving models of

Keenan Crane is assistant professor of computer science at Carnegie Mellon University. His e-mail address is kmcrane@cs . cmu . edu.

Max Wardetzky is professor of mathematics at University of Göttingen. His e-mail address is wardetzky@math . uni-goettingen .de.

For permission to reprint this article, please contact:

reprint-permission@ams.org.

DOI: http://dx.doi.org/10.1090/noti1578

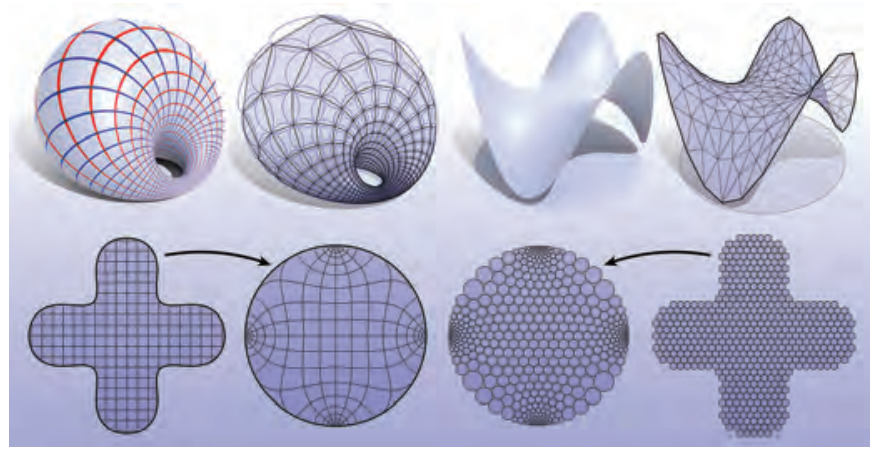

Figure 1. Discrete differential geometry reimagines classical ideas from differential geometry without reference to differential calculus. For instance, surfaces parameterized by principal curvature lines are replaced by meshes made of circular quadrilaterals (top left), the maximum principle obeyed by harmonic functions is expressed via conditions on the geometry of a triangulation (top right), and complex-analytic functions can be replaced by so-called circle packings that preserve tangency relationships (bottom). These discrete surrogates provide a bridge between geometry and computation, while at the same time preserving important structural properties and theorems.

discrete geometry seek to exactly preserve global geometric invariants such as total curvature. More broadly, DDG focuses on the discretization of objects that do not naturally fall under the umbrella of traditional numerical analysis. This article provides an overview of some of the themes in DDG. 


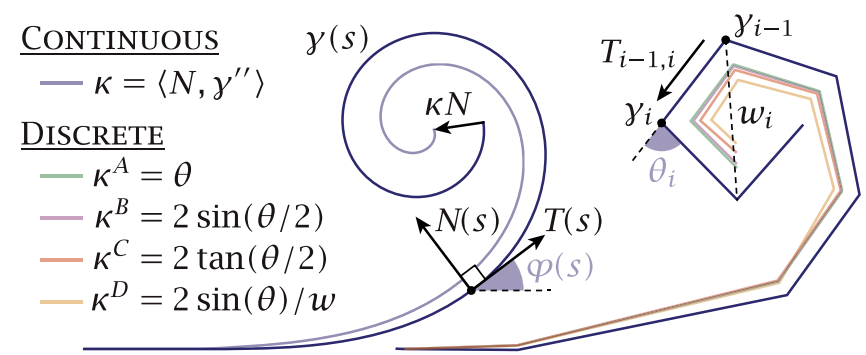

Figure 2. A given geometric quantity from the smooth setting, like curvature $\kappa$, may have several reasonable definitions in the discrete setting. Discrete differential geometry seeks definitions that exactly replicate properties of their smooth counterparts.

\section{The Game}

Our article is organized around a "game" often played in discrete differential geometry in order to come up with a discrete analogue of a given smooth object or theory:

(1) Write down several equivalent definitions in the smooth setting.

(2) Apply each smooth definition to an object in the discrete setting.

(3) Analyze trade-offs among the resulting discrete definitions, which are invariably inequivalent.

Most often, none of the resulting discrete objects preserve all the properties of the original smooth one-a socalled no free lunch scenario. Nonetheless, the properties that are preserved often prove invaluable for particular applications and algorithms. Moreover, this activity yields some beautiful and unexpected consequences-such as a connection between conformal geometry and pure combinatorics, or a description of constant-curvature surfaces that requires no definition of curvature! To highlight some of the challenges and themes commonly encountered in DDG, we first consider the simple example of the curvature of a plane curve.

\section{Discrete Curvature of Planar Curves}

How do you define the curvature of a discrete curve? For a smooth arc-length parameterized curve $\gamma(s):[0, L] \rightarrow \mathbb{R}^{2}$, curvature $\kappa$ is classically expressed in terms of second derivatives. In particular, if $\gamma$ has unit tangent $T:=\frac{d}{d s} \gamma$ and unit normal $N$ (obtained by rotating $T$ a quarter turn in the counter-clockwise direction), then

$$
\kappa:=\left\langle N, \frac{d^{2}}{d s^{2}} \gamma\right\rangle=\left\langle N, \frac{d}{d s} T\right\rangle .
$$

Suppose instead we have a polygonal curve with vertices $\gamma_{1}, \ldots, \gamma_{n} \in \mathbb{R}^{2}$, as often used for numerical computation (see Figure 2, right). Here we hit upon the most elementary problem of discrete differential geometry: discrete geometric objects are often not sufficiently differentiable (in the classical sense) for standard definitions to apply. For instance, our curvature definition (Equation 1) causes trouble, since at vertices our discrete curve is not twice differentiable, nor does it have well defined normals. The basic approach of DDG is to find alternative characterizations in the smooth setting that can be applied to discrete geometry in a natural way. With curvature, for instance, we can apply the fundamental theorem of calculus to Equation 1 to acquire a different statement: if $\varphi$ is the angle from the horizontal line to the unit tangent $T$, then

$$
\int_{a}^{b} \kappa d s=\varphi(b)-\varphi(a) \bmod 2 \pi .
$$

Put more simply: curvature is the rate at which the tangent turns. This characterization can be applied naturally to our polygonal curve: along any edge the change in angle is clearly zero. At a vertex it is simply the turning angle $\theta_{i}:=\varphi_{i, i+1}-\varphi_{i-1, i}$ between the directions $\varphi_{i-1, i}, \varphi_{i, i+1}$ of the two incident edges, yielding our first notion of discrete curvature:

$$
\kappa_{i}^{A}:=\theta_{i} \in(-\pi, \pi) .
$$

Are there other characterizations that also lead naturally to a discrete formulation? Yes: for instance we can consider the motion of $\gamma$ that most quickly reduces its length. In the smooth case it is well known that the change in length with respect to a smooth variation $\eta(s):[0, L] \rightarrow \mathbb{R}^{2}$ that vanishes at the endpoints of the curve is given by integration against curvature:

$$
\left.\frac{d}{d \varepsilon}\right|_{\varepsilon=0} \text { length }(\gamma+\varepsilon \eta)=-\int_{0}^{L}\langle\eta(s), \kappa(s) N(s)\rangle d s .
$$

Hence, the velocity that most quickly reduces length is $\kappa N$. For a polygonal curve, we can simply differentiate the sum of the edge lengths $L:=\sum_{i=1}^{n-1}\left|\gamma_{i+1}-\gamma_{i}\right|$ with respect to any vertex position. At a vertex $i$ we obtain

$$
\partial_{\gamma_{i}} L=\frac{\gamma_{i}-\gamma_{i-1}}{\left|\gamma_{i}-\gamma_{i-1}\right|}-\frac{\gamma_{i+1}-\gamma_{i}}{\left|\gamma_{i+1}-\gamma_{i}\right|}=: T_{i-1, i}-T_{i, i+1},
$$

i.e., just a difference of unit tangent vectors $T_{i, i+1}$ along consecutive edges. If $N_{i} \in \mathbb{R}^{2}$ is the unit angle bisector at vertex $i$, this difference can also be expressed as

$$
\kappa_{i}^{B} N_{i}:=2 \sin \left(\theta_{i} / 2\right) N_{i},
$$
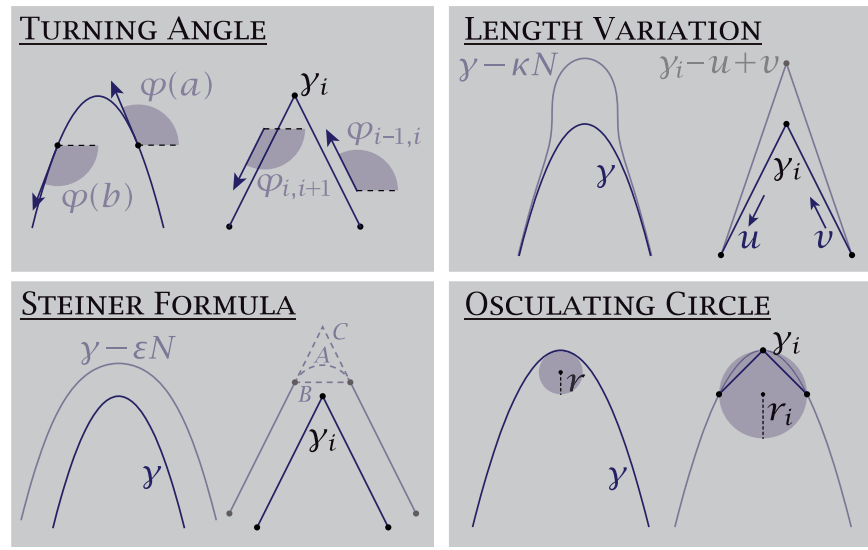

Figure 3. Different characterizations of curvature in the smooth setting naturally lead to different notions of discrete curvature. (Here we abbreviate $T_{i, i+1}$ and $T_{i-1, i}$ by $u$ and $v$, respectively.) 
providing a discretization of the curvature normal $\kappa N$. A closely-related idea is to consider how the length of a curve changes if we displace it by a small constant amount in the normal direction. As observed by Steiner, the new length of a smooth curve can be expressed as

$$
\operatorname{length}(\gamma+\varepsilon N)=\operatorname{length}(\gamma)-\varepsilon \int_{0}^{L} \kappa(s) d s \text {. }
$$

Since this formula holds for any small piece of the curve, it can be used to obtain a notion of curvature at each point. How do we define normal offsets in the polygonal case? At vertices we again encounter the issue that we have no notion of normals. One idea is to break the curve into individual edges which can then be translated by $\varepsilon$ along their respective normal directions. We can then close the gaps between edges in a variety of ways: using (A) a circular arc of radius $\varepsilon$, (B) a straight line, or by (C) extending the edges until they intersect (see Figure 3, bottom left). If we then calculate the lengths for these new curves, we get

$$
\begin{aligned}
& \text { length }_{A}=\operatorname{length}(\gamma)-\varepsilon \sum_{i=2}^{n-1} \theta_{i}, \\
& \text { length }_{B}=\operatorname{length}(\gamma)-\varepsilon \sum_{i=2}^{n-1} 2 \sin \left(\theta_{i} / 2\right), \\
& \text { length }_{C}=\operatorname{length}(\gamma)-\varepsilon \sum_{i=2}^{n-1} 2 \tan \left(\theta_{i} / 2\right) .
\end{aligned}
$$

Mirroring the observation in the smooth setting, we can now say that whatever change we observe in the length provides a definition for discrete curvature. The first two are the same as ones we have seen already: the circular arc yielding the expression from Equation 2, and the straight line corresponding to Equation 4. The third one provides yet another notion of discrete curvature

$$
\kappa_{i}^{C}:=2 \tan \left(\theta_{i} / 2\right) \text {. }
$$

Finally, in the smooth case it is also well known that curvature has magnitude equal to the inverse of the radius of the so-called osculating circle, which agrees with the curve up to second order. A natural way to define an osculating circle for a polygon is to take the circle passing through a vertex and its two neighbors. From the formula for the radius $R_{i}$ of a circumcircle in terms of the side lengths of the corresponding triangle, one easily gets a discrete curvature that is different from the ones we saw before:

$$
\kappa_{i}^{D}:=1 / R_{i}=2 \sin \left(\theta_{i}\right) / w_{i},
$$

where $w_{i}:=\mid \gamma_{i+1}-$ $\gamma_{i-1} \mid$. Apart from merely being different expressions, we can notice that $\kappa^{A}, \kappa^{B}$, and $\kappa^{C}$ are all invariant under a uniform scaling of the curve, whereas $\kappa^{D}$ scales like the smooth curvature $\kappa$. This situation demonstrates another common phenomenon in discrete

$$
\begin{gathered}
\text { The fundamental } \\
\text { behavior of } \\
\text { geometry is } \\
\text { neither inherently } \\
\text { smooth nor } \\
\text { discrete. }
\end{gathered}
$$

differential geometry, namely that depending on which smooth characterization is used as a starting point, one may end up with pointwise or integrated quantities in the discrete case.

As one might imagine, there are many other possible starting points for obtaining a discrete analogue of curvature. Eventually, however, all starting points end up leading back to the same definitions, suggesting that there may be only so many possibilities. For example, if $\phi: \mathbb{R}^{2} \rightarrow \mathbb{R}$ is the signed distance from a smooth closed curve $\gamma$, then applying the Laplacian $\Delta$ yields the curvature of its level curves; in particular, $\left.\Delta \phi\right|_{\phi=0}$ yields the curvature of $\gamma$. Likewise, if we apply the Laplacian to the signed distance function for a discrete curve, we recover $\kappa^{A}$ on one side and $\kappa^{B}$ on the other. Yet another approach is the theory of normal cycles (as discussed by Morvan), related to the Steiner formula from Equation 5. Here, rather than settle on a single normal $N_{i}$ at each vertex we consider all unit vectors between the unit normals of the two incident edges, ultimately leading back to the first discrete curvature $\kappa^{A}$. The theory of normal cycles applies equally well to both smooth and polygonal curves, again reinforcing the perspective that the fundamental behavior of geometry is neither inherently smooth nor discrete, but can be well captured in both settings by picking the appropriate ansatz. More broadly, the fact that equivalent characterizations in the smooth setting lead to different inequivalent definitions in the discrete setting is not special to the case of curves, but is one of the central themes in discrete differential geometry.

From here, a natural question arises: which discrete curvature is "best"? A traditional criterion for discriminating among different discrete versions is the question of convergence: if we consider finer and finer approximating polygons, will our discrete curvatures converge to the classical smooth one? However, convergence does not always single out a best version: treated appropriately, all four of our discrete curvatures will converge. We must

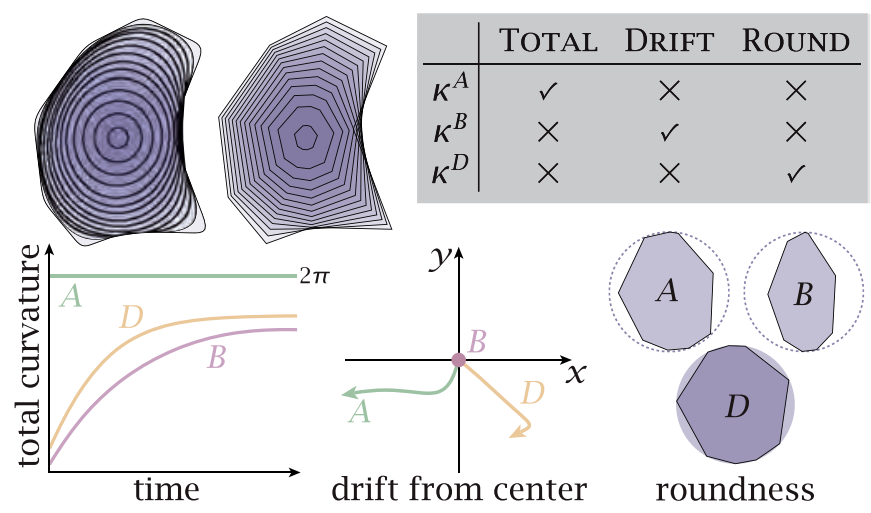

Figure 4. Typically, not all properties of a smooth object can be preserved exactly at the discrete level. For curve-shortening flow, for example, $\kappa^{A}$ exactly preserves the total curvature, $\kappa^{B}$ exactly preserves the center of mass, and with $\kappa^{D}$ the flow remains stationary (up to rescaling) for any circular solution. However, no local definition of discrete curvature can provide all three properties simultaneously. 
therefore look beyond convergence, toward exact preservation of properties and relationships from the smooth setting. Which properties should we try to preserve? The answer of course depends on what we aim to use these curvatures for.

As a toy example, consider the curve-shortening flow (depicted in Figure 4, top left), where a curve evolves according to the velocity that most quickly reduces its length. As discussed above, this velocity is equal to the curvature normal $\kappa N$. A smooth, simple curve evolving under this flow exhibits several basic properties: it has at all times total curvature $2 \pi$, its center of mass remains fixed, it tends toward a circle of vanishing radius, and remains embedded for all time, i.e., no selfcrossings arise (Gage-Grayson-Hamilton). Do our discrete curvatures furnish these same properties? A numerical experiment is shown in Figure 4. Here we evolve our polygon by a simple time-discrete flow $\gamma_{i} \leftarrow \gamma_{i}+\tau \kappa_{i} N_{i}$ with a fixed time step $\tau>0$. For $\kappa^{D}, N_{i}$ is the unit vector along the circumradius; otherwise it is the unit angle bisector. Not surprisingly, $\kappa^{A}$ preserves total curvature (due to the fundamental theorem of calculus); $\kappa^{B}$ does not drift (consider summing Equation 3 over all vertices); and $\kappa^{D}$ has circular polygons as limit points (since all velocities point toward the center of a common circle). However, no discrete curvature satisfies all three properties simultaneously. Moreover, for a constant time step $\tau$ no such flow can guarantee that new crossings do not occur. This situation illustrates the no free lunch idea: no matter how hard we try, we cannot find a single discrete object that preserves all the properties of its smooth counterpart. Instead, we have to pick and choose the properties best suited to the task at hand.

Suppose that instead of curvature flow, we consider two other beautiful topics in the geometry of plane curves: the Whitney-Graustein theorem, which classifies regular homotopy classes of curves by their total curvature, and Kirchhoff's famous analogy between motions of a spherical pendulum and elastic curves, i.e., curves that extremize the bending energy $\int_{0}^{L} \kappa^{2} d s$ subject to boundary conditions. Among the curvatures discussed above, only $\kappa^{A}$ provides a discrete version of WhitneyGraustein, but does not provide an exact discrete analogue of Kirchhoff. Likewise, $\kappa^{C}$ preserves the structure of the Kirchhoff analogy, but not Whitney-Graustein. This kind of no free lunch situation is a characteristic feature of DDG. A similar obstacle is encountered in the theory of ordinary differential equations, where it is known that there are no numerical integrators for Hamiltonian systems that simultaneously conserve energy, momentum, and the symplectic form. From a computational point of view, making judicious choices about which quantities to preserve for which applications goes hand-in-hand with providing formal guarantees on the reliability and robustness of algorithms.

We now give a few glimpses into recent topics and trends in DDG.

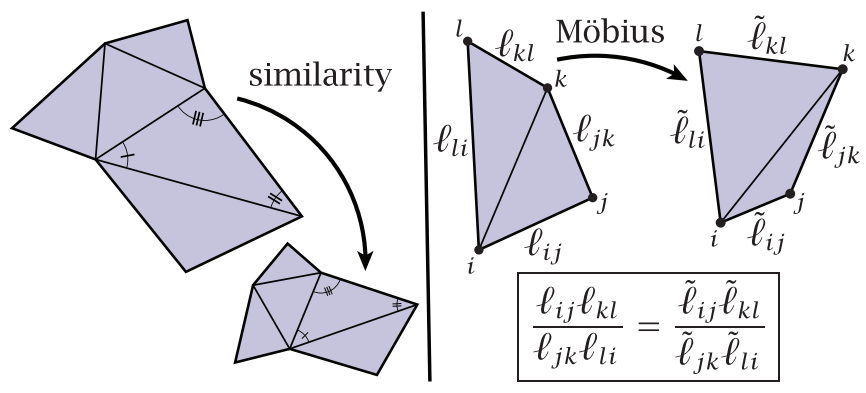

Figure 5. What is the simplicial analogue of a conformal map? Requiring all angles to be preserved is too rigid, forcing a global similarity (left). Asking only for preservation of so-called length cross ratios provides just the right amount of flexibility, maintaining much of the structure found in the smooth setting such as invariance under Möbius transformations (right).

\section{Discrete Conformal Geometry}

A conformal map is, roughly speaking, a map that preserves angles (see Figure 1, bottom left). A good example is Mercator's projection of the globe: even though area gets stretched out badly-making Greenland look much bigger than Australia!- the directions "north" and "east" remain at right angles, which is very helpful if you are trying to navigate the sea. A beautiful fact about conformal maps is that any surface can be conformally mapped to a space of constant curvature ("uniformization"), providing it with a canonical geometry. This fact, plus the fact that conformal maps can be efficiently computed (e.g., by solving sparse linear systems), have led in recent years to widespread development of conformal mapping algorithms as a basic building block for digital geometry processing algorithms. In applications, discrete conformal maps are used for everything from sensor network layout to comparative analysis of medical or anatomical data. Of course, to process real data one must be able to compute conformal maps on discrete geometry.

What does it mean for a discrete map to be conformal? As with curvature, one can play the game of enumerating several equivalent characterizations in the smooth setting. Consider for instance a map $f: M \rightarrow D^{2} \subset \mathbb{C}$ from a disklike surface $M$ with Riemannian metric $g$ to the unit disk $D^{2}$ in the complex plane. This map is conformal if it too few degrees of it preserves infinitesitoo few degrees of mal circles, if it can freedom relative to be expressed as a pair $f$ of real conjugate har the number of constraints monic functions $f=$ $a+b i$, if it is a critical point of the Dirichlet energy $\int_{M}|d f|^{2} d A$, or

if it induces a new metric $\tilde{g}:=d f \otimes d f$ that at each point is a positive rescaling of the original one: $\tilde{g}=e^{2 u} g$. Each starting point leads down a path toward different 
consequences in the discrete setting, and to algorithms with different computational tradeoffs.

Oddly enough, the most elementary characterization of conformal maps, angle preservation, does not translate very well to the discrete setting (see Figure 5). Consider for instance a simplicial map that takes a triangulated disk $K=(V, E, F)$ to a triangulation in the plane. Any map that preserves interior angles will be a similarity on each triangle, i.e., it can only rigidly rotate and scale. But since adjacent triangles share edges, the scale factor for all triangles must be identical. Hence, the only discrete surfaces that can be conformally flattened in this sense are those that are (up to global scale) developable, i.e., that can be rigidly unfolded into the plane. This outcome is in stark contrast to the smooth setting, where any disk can be conformally flattened. This situation reflects a common scenario in DDG: rigidity, or what in finite element analysis is sometimes called locking. There are simply too few degrees of freedom relative to the number of constraints: we want to match angles at all $3 F$ corners, but have only $2 V<3 F$ degrees of freedom. Hence, if we insist on angle preservation we have no chance of capturing the flexibility of smooth conformal maps.

Other characterizations provide greater flexibility. One idea is to associate each vertex of our discrete disk $K$ with a circle in the plane. A theorem of Koebe implies that one can always arrange these circles such that two circles are tangent if they belong to a shared edge and all boundary circles are tangent to a common circle bounding the rest. For a regular triangular lattice approximating a region $U \subset \mathbb{C}$, Thurston noticed that this map approximates a smooth conformal map $f: U \rightarrow D^{2}$ as the region is filled by smaller and smaller circles (see Figure 1, bottom), as later proved by Rodin and Sullivan. Unlike a traditional finite element discretization, these so-called circle packings also preserve many of the basic structural properties of conformal maps. For instance, composition with a Möbius transformation of the disk yields another uniformization map, as in the smooth setting. More broadly, circle packings provide an unexpected bridge between geometry and combinatorics, since the geometry of a map is determined entirely by incidence relationships. ${ }^{1}$ On the flip side, this means a different theory is needed to account for the geometry of irregular triangulations, as more commonly used in applications.

An alternative theory starts from the idea that under a conformal map the Riemannian metric $g$ experiences a uniform scaling at each point: $\tilde{g}=e^{2 u} g$. In other words, vectors tangent to a given point $p \in M$ shrink or grow by a positive factor $e^{u}$. In the simplicial setting $g$ is replaced by a piecewise Euclidean metric, i.e., a collection of positive edge lengths $\ell: E \rightarrow \mathbb{R}_{>0}$ that satisfy the triangle inequality in each face. Two such metrics $\ell, \tilde{\ell}$ are then said to be discretely conformally equivalent if they are related by $\tilde{\ell}_{i j}=e^{\left(u_{i}+u_{j}\right) / 2} \ell_{i j}$ for any collection of discrete scale factors $u: V \rightarrow \mathbb{R}$. Though at first glance this

${ }^{1}$ See "Circle Packing" in the December 2003 Notices www. ams . org/notices/200311/fea-stephenson.pdf. relationship looks like a simple numerical approximation, it turns out to provide a complete discrete theory that preserves much of the structure found in the smooth setting, with close ties to theories based on circles. An equivalent characterization is the preservation of length cross ratios $\mathfrak{c}_{i j k l}:=\ell_{i j} \ell_{k l} / \ell_{j k} \ell_{l i}$ associated with each edge $\in E$; for a mesh embedded in $\mathbb{R}^{n}$ these ratios are invariant under Möbius transformations, again mimicking the smooth theory. This theory also leads to efficient, convex algorithms for discrete Ricci flow, which is a starting point for many applications in digital geometry processing.

More broadly, discrete conformal geometry and discrete complex analysis is an active area of research, with elegant theories not only for triangulations but also for lattice-based discretizations, which make contact with the topic of (discrete) integrable systems, discussed below. Yet basic questions about properties like convergence, or descriptions that are compatible with extrinsic geometry, are still only starting to be understood.

\section{Discrete Differential Operators}

Differential geometry and in particular Riemannian manifolds can be studied from many different perspectives. In contrast to the purely geometric perspective (based on, say, notions of distance or curvature), differential operators provide a very different point of view. One of the most fundamental operators in both physics and geometry is the Laplace-Beltrami operator $\Delta$ (or Laplacian for short) acting on differential $k$-forms. It describes, for example, heat diffusion, wave propagation, and steady state fluid flow, and is key to the Schrödinger equation in quantum mechanics. It also provides a link between analytical and topological information: for instance, on closed Riemannian manifolds the dimension of harmonic $k$-forms (i.e., those in the kernel of $\Delta$ ) equals the dimension of the $k$ th cohomology - a purely topological quantity. The spectrum of the Laplacian (i.e., the list of eigenvalues) likewise reveals a great deal about the geometry of the manifold. For example, the first nonzero eigenvalue of the 0 -form Laplacian provides an upper and a lower bound on optimally cutting a compact Riemannian manifold $M$ into two disjoint pieces of, loosely speaking, maximal volume and minimal perimeter (Cheeger-Buser). These so-called Cheeger cuts have a wide range of applications across machine learning and computer vision; more broadly, eigenvalues and eigenfunctions of $\Delta$ help to generalize traditional Fourier-based simulation and signal processing to more general manifolds.

These observations motivate the study of discrete Laplacians, which can be defined even in the purely combinatorial setting of graphs. Here we briefly outline their definition for orientable finite simplicial $n$-manifolds, such as polyhedral surfaces, without boundary. Our exposition is similar to what has become known as discrete exterior calculus. To this end, consider the simplicial boundary operators $\partial_{k}: C_{k} \rightarrow C_{k-1}$ acting on $k$-chains (i.e., formal linear combinations of $k$-simplices). The corresponding dual spaces (cochains) $C^{k}:=\operatorname{Hom}\left(C_{k}, \mathbb{R}\right)$ and 
respective dual operators $\delta_{k}: C^{k} \rightarrow C^{k+1}$ give rise to the chain complex

$$
\{0\} \rightarrow C^{0} \rightarrow C^{1} \rightarrow \ldots \rightarrow C^{n} \rightarrow\{0\} .
$$

The chain property says that $\delta_{k} \circ \delta_{k-1}=0$, and one hence obtains simplicial cohomology $H^{k}:=\operatorname{ker}\left(\delta_{k}\right) / \operatorname{im}\left(\delta_{k-1}\right)$. To define a Laplacian in this setting, we equip each $C^{k}$ with a positive definite inner product $(\cdot, \cdot)_{k}$, and let $\delta_{k}^{*}$ be the adjoint operator with respect to these inner products, i.e., $\left(\delta_{k} \alpha, \beta\right)_{k+1}=\left(\alpha, \delta_{k}^{*} \beta\right)_{k}$ for all $\alpha, \beta$. The Laplacian on $k$-cochains is then defined as

$$
\Delta_{k}:=\delta_{k}^{*} \delta_{k}+\delta_{k-1} \delta_{k-1}^{*}
$$

The resulting space of harmonic $k$-cochains, $\{\alpha \in$ $\left.C^{k} \mid \Delta_{k} \alpha=0\right\}$ is then isomorphic to $H^{k}$-just as in the smooth setting. This fact is independent of the choice of inner product, mirroring the fact that cohomology depends only on topological structure. Likewise, for any inner product one obtains a discrete Hodge decomposition

$$
C^{k}=\operatorname{ker}\left(\Delta_{k}\right) \oplus \operatorname{im}\left(\delta_{k-1}\right) \oplus \operatorname{im}\left(\delta_{k}^{*}\right),
$$

where here the subspaces do depend on the choice of inner product.

At this point we return again to the game of DDG: which choice of inner product is best? A trivial inner product leads to purely combinatorial graph Laplacians, which do not (in general) converge to their smooth counterparts (e.g., when approximating a smooth manifold by a polyhedral one). Another choice is to consider linear interpolation of $k$-cochains over $n$-dimensional simplices, resulting in what is known as Whitney elements. For $n=2$, we get the so-called cotan Laplacian (Pinkall and Polthier), which is widely used in digital geometry processing. Though other choices are possible, we again encounter a no free lunch situation: no choice of inner product can preserve all the properties of the smooth Laplacian. Which properties do we care about? Beyond convergence, perhaps the most desirable properties are the maximum principle (which ensures, for instance, proper behavior for heat flow), and the property that, for flat domains, linear functions are in the kernel (leading to a proper definition of barycentric coordinates). For general unstructured meshes there are no discrete Laplacians with all of these properties. However, certain types of meshes (such as weighed Delaunay triangulations) do indeed allow for "perfect" discrete Laplacians, offering a connection between geometry and (discrete) differential operators.

\section{Discrete Integrable Systems}

Another topic that has provided inspiration for many ideas in DDG is parameterized surface theory. Consider for instance the problem of dressing a given surface by a fishnet stocking, i.e., a woven material composed of inextensible yarns following transversal "warp" and "weft" directions (see Figure 6, right). This task corresponds to decorating a surface with a tiling where each vertex is incident to four parallelograms. Infinitesimally, such a tiling is known as a weak Chebyshev net (Chebyshev 1878), and locally corresponds to a regularly parameterized surface $f: U \subset \mathbb{R}^{2} \rightarrow \mathbb{R}^{3}$ where the directional derivatives $f_{u}$ and $f_{v}$ along the coordinate directions satisfy $\left|f_{u}\right|_{v}=$ $\left|f_{v}\right|_{u}=0$, i.e., partial derivatives with respect to one parameter have constant length along the parameter lines of the other parameter. The special case of rhombic tilings $\left(\left|f_{u}\right|=\left|f_{v}\right|=1\right)$ are known as (strong) Chebyshev nets. Can every smooth surface be wrapped in a stocking? Locally (i.e., in a small patch around any given point) the answer is "yes." Globally, however, there are severe obstructions to doing so, which provide some fascinating connections to physics.

Consider for instance the special case of so-called $K$-surfaces, characterized by constant Gauß curvature $K=-1$. Every K-surface admits a parameterization $f$ : $U \subset \mathbb{R}^{2} \rightarrow \mathbb{R}^{3}$ aligned with the two transversal asymptotic directions along which normal curvature vanishes. Hence, if $N$ is the unit surface normal then

$$
\left\langle f_{u u}, N\right\rangle=\left\langle f_{v v}, N\right\rangle=0 .
$$

Asymptotic parameterizations are weak Chebyshev nets since

$$
\text { (8) } \quad a:=\left|f_{u}\right|, b:=\left|f_{v}\right| \quad \text { satisfy } a_{v}=b_{u}=0 \text {. }
$$

Moreover, one can show that the angle $\phi$ between asymptotic lines satisfies the sine-Gordon equation

$$
\phi_{u v}-a b \sin \phi=0,
$$

and conversely, every solution to the sine-Gordon equation describes a parameterized K-surface. Hilbert used this equation (and Chebyshev nets) to prove that the complete hyperbolic plane cannot be embedded isometrically into $\mathbb{R}^{3}$. More generally, the sine-Gordon equation has attracted much interest both in mathematics as an example of an infinite-dimensional integrable system, and in physics as an example of a system that admits remarkably stable soliton solutions, akin to waves that travel uninterrupted all the way across the ocean. Another key property of the sine-Gordon equation is the existence of a so-called spectral parameter $\lambda>0$ : Equation 9 is invariant under a rescaling $a \rightarrow \lambda a$ and $b \rightarrow \lambda^{-1} b$, giving rise to a oneparameter associated family of K-surfaces. Geometrically, the parameter $\lambda$ rescales the edges of parallelograms while preserving the angle between asymptotic lines.

Do these properties depend critically on the smooth nature of the solutions, or can they also be faithfully captured in the discrete setting? Hirota derived such a discrete version without any reference to geometry. Later Bobenko and Pinkall suggested a geometric definition of discrete K-surfaces that recovers Hirota's equation. In their setting, discrete K-surfaces are defined as discrete (weak) Chebyshev nets with the additional property that all four edges incident to any vertex lie in a common plane. The last requirement is a natural discrete analogue of Equation 7. This definition of discrete K-surfaces also comes with a spectral parameter $\lambda$ and results in Hirota's discrete sine-Gordon equation-without requiring any notion of discrete Gauß curvature. Only recently has a discrete version of Gauß curvature been suggested that results in discrete $\mathrm{K}$-surfaces indeed having constant negative Gauß curvature. 

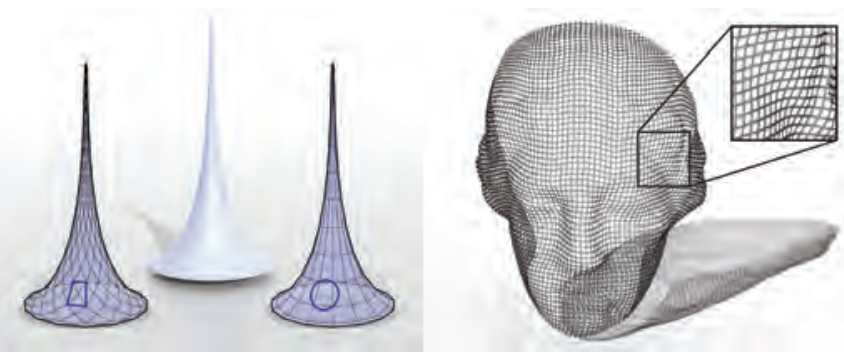

Figure 6. Left: two discrete parameterizations of a pseudosphere (constant Gauß curvature $K=-1$ ), one with a Chebychev net along asymptotic directions (left) and another along principal curvature lines (right). Right: a discrete Chebyshev net on a surface of varying curvature, resembling the weft and warp directions of a woven material.

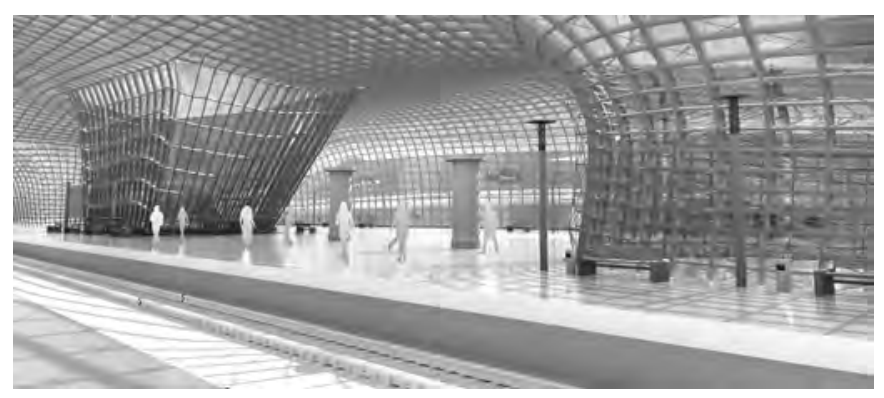

Figure 7. Discrete parameterized surfaces play a role in architectural geometry, where special incidence relationships on quadrilaterals translate to manufacturing constraints like zero nodal torsion, or offset surfaces of constant thickness. Here a curvature line parameterized surface discretized by a conical net is used in the design of a railway station.

For discrete K-surfaces with all equal edge lengths (i.e., the rhombic case) the four neighboring vertices of a given vertex must lie on a common circle. By considering a subset of the diagonals of the quadrilaterals, one obtains another quad mesh with the property that all quads have a circumscribed circle, resulting in socalled cK-nets (see Figure 6, left). In the discrete setting, regular networks of circular quadrilaterals play the role of curvature line parameterized surfaces (as in Figure 1 , top left). This transformation therefore mimics the smooth setting, where the angle bisectors of asymptotic lines are lines of principal curvature. More broadly, the theory of quad nets with special incidence relationships is closely linked to physical manufacturing considerations in the field of architectural geometry. For example, a quad net is conical if the four quads around each vertex are tangent to a common cone-such surfaces admit face offsets of constant width, making them attractive for the construction of (for instance) glass-paneled structures, as in Figure 7.

For further reading, see Discrete Differential Geometry (2008, Alexander Bobenko ed.).

\section{Image Credits}

Figures 1-6 courtesy of Keenan Crane and Max Wardetzky. Figure 7 courtesy of B. Schneider.

Author photo of Keenan Crane courtesy of Keenan Crane. Author photo of Max Wardetzky courtesy of Max Wardetzky.

\section{ABOUT THE AUTHORS}

Keenan Crane works at the interface between differential geometry and geometric algorithms. His scientific career started out studying Pluto-back when it was still a planet!

Max Wardetzky's interest in discrete differential geometry comes from his outstanding and truly inspiring teachers in differential geometry and his exposure to marvelous people in computer graphics.

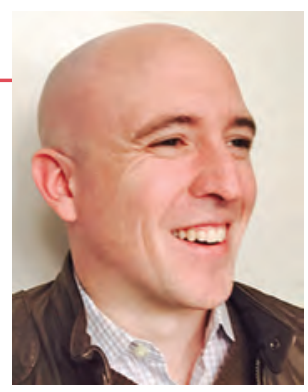

Keenan Crane

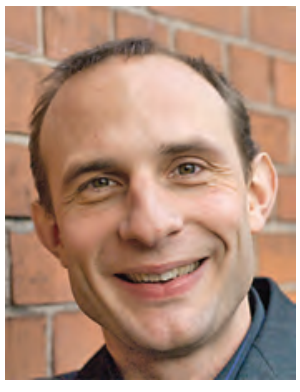

Max Wardetzky 


\title{
Immigration, Freedom, and the History of the Institute for Advanced Study
}

\author{
Fadi Bardawil', Thomas Dodman', Ian Jauslin³, Pascal Marichalar 4 \\ Klaus Oschema ${ }^{5}$, and Peter Redfield ${ }^{6}$
}

\author{
Communicated by Harriet Pollatsek
}

In response to the January 27, 2017, US presidential executive order initially banning travel and immigration from seven predominantly Muslim countries, members of the Institute for Advanced Study (IAS) mobilized a History Working Group. This article is adapted from their reports in the spring 2017 IAS Newsletter. The full articles, as well as a companion exhibit showcasing original materials from the Institute's archives, are also available on the IAS website $^{7}$ and in print/ebook as a booklet.

\footnotetext{
${ }_{1}^{1}$ Fadi Bardawil is an anthropologist at the University of North Carolina at Chapel Hill, and a former Member (2016-2017) of the School of Social Science at the IAS. His e-mail address is bardawi 1 @ emai 1 . unc. edu

${ }^{2}$ Thomas Dodman is a historian at Columbia University, and a former Member (2016-2017) of the School of Historical Studies at the IAS. His e-mail address is td2551@columbia. edu

${ }^{3}$ Ian Jauslin is a Member (2016-2017) of the School of Mathematics at the IAS. His e-mail address is jaus1 in@ias.edu

${ }^{4}$ Pascal Marichalar is a sociologist and historian with the French $\mathrm{Na-}$ tional Center for Scientific Research (CNRS), and a former Member (2016-2017) of the School of Social Science, IAS. His e-mail address is pascal.marichalar@cnrs.fr

${ }^{5}$ Klaus Oschema is a historian at the Ruhr-Universität Bochum, and a former Member (2016-2017) of the School of Historical Studies at the IAS. His e-mail address is $\mathrm{k} 1$ aus.oschema@ruhr-universitaet-bochum. de

${ }^{6}$ Peter Redfield is an anthropologist at the University of North Carolina at Chapel Hill, and a former Member (2016-2017) of the School of Social Science at the IAS. His e-mail address is redfield@unc. edu
}

7 www.ias.edu/idea-tags/history-working-group

For permission to reprint this article, please contact:

reprint-permission@ams .org.

DOI: http://dx.doi.org/10.1090/noti1596

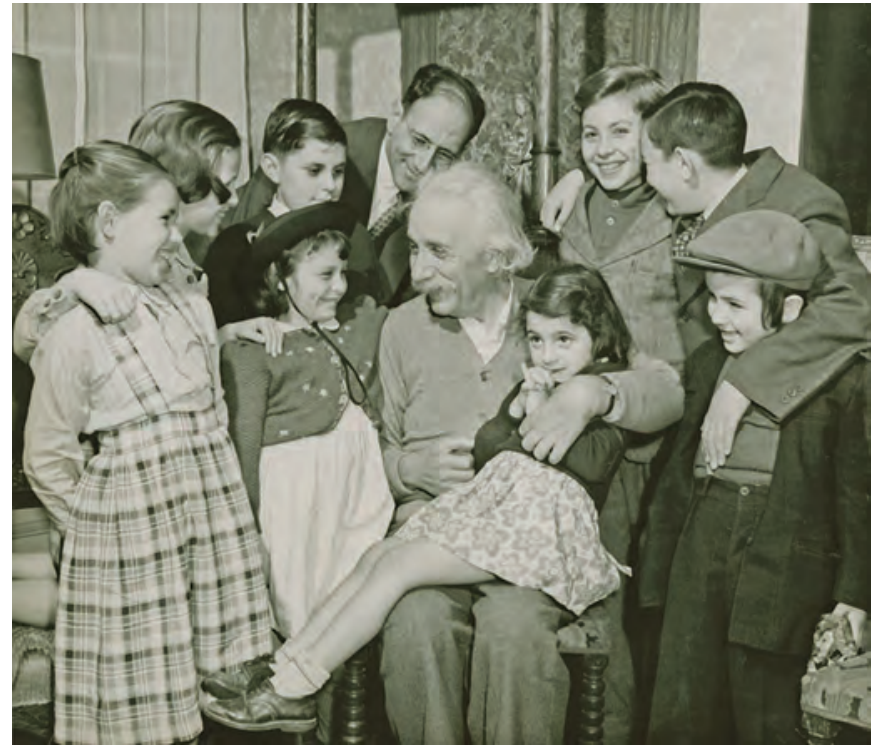

Figure 1: Albert Einstein at his home, 112 Mercer Street in Princeton, New Jersey, with a group of World War II European Jewish refugee children.

\section{The Institute's Founding Ethos in Our Precarious Present}

The Institute for Advanced Study came into being at the most inauspicious of times. Founded in the early years of the Great Depression, it took shape during the buildup to the Second World War and under the growing shadow of authoritarian regimes. Its first director, Abraham Flexner, published his manifesto on "The Usefulness of Useless Knowledge" in October 1939, barely a month after the outbreak of hostilities in Europe. Surely this was a daunting moment to defend "the fearless and irresponsible thinker" 
and advocate for the free expression of knowledge and curiosity.

The very adversity of the era, however, created opportunities for the fledgling institution, primarily in the form of sudden availability of renowned and newly mobile scholars from the upper echelons of the German university system. After expressing initial hesitation, Flexner followed the urging of influential faculty members, particularly the mathematician Oswald Veblen, in seeking to provide a haven for some of these new refugees, a sanctuary tradition continued by Frank Aydelotte, Flexner's successor. In association with the Emergency Committee in Aid of Displaced Foreign Scholars, the Rockefeller Foundation, and the Carnegie Foundation, the IAS played a leading role in this farsighted, if ever elite, rescue effort.

We find ourselves today, nearly nine decades after the Institute's founding in 1930, at another inauspicious juncture. Global political forces in power from Turkey to the United States are posing serious threats to the autonomy of scientific research and the mobility of researchers, undercutting two cardinal conditions for scientific progress. Walls, fences, bans, blocks, restrictions, cuts, and expulsions are slowly becoming run-of-the-mill terms for us to navigate in an increasingly precarious political landscape.

Travel restrictions involving pure accidents of birth, documented by passports from flagged countries, have prompted us to revisit today the Institute's history. We seek to provide sketches of earlier scholarly lives and scientific cultures interrupted by nationalist forces of exclusion. That these lives and cultures managed to reconstitute themselves and enrich our common human heritage is thanks to efforts to provide them with sanctuary.

\section{The Conversion of Abraham Flexner}

At the end of January 1933, Adolf Hitler became chancellor of Germany. Over the next two months, the Nazi Party moved quickly to consolidate its power, expanding executive authority through emergency decrees and swiftly moving into a formal dictatorship. An edict in April initiated a purge of civil servants who were of non-Aryan descent or exhibited suspect political sentiments. This law directly impacted German universities, and it had a particularly strong effect in the fields of mathematics and natural sciences, where Jews had enjoyed better prospects of pursuing a scholarly career. As a consequence, many of the country's strongest intellectual centers lost leading figures in the space of just a few months, including the renowned Mathematical Institute at the University of Göttingen, home of David Hilbert, Richard Courant, Hermann Weyl, and Emmy Noether. This upheaval produced a sudden wave of refugee scholars seeking to emigrate and desperate to find positions elsewhere. It also coincided with the transition of IAS from a concept to an embodied institution, through the founding of the inaugural School of Mathematics.

The crisis of refugee scholars presented Flexner and Veblen with a challenging opportunity. The Institute had already pulled an extraordinary coup in recruiting Albert Einstein and John von Neumann shortly before Hitler's coming to power. But how far should they continue in recruiting additional émigrés? Flexner initially expressed ambivalence on the topic, torn between a desire to live up to founding ideals and concern over the need to support and foster local talent. He wrote to Veblen on March 27, 1933: "Mr. Bamberger and Mrs. Fuld were very anxious from the outset that no distinction should be made as respects race, religion, nationality, etc., and of course I am in thorough sympathy with their point of view, but on the other hand if we do not develop America, who is going to do it, and the question arises how much we ought to do for others and how much to make sure that civilization in America advances." On May 2, again responding to Veblen, he expanded on the same theme: "We are certainly in the devil of a fix. Unable to care for our own younger men, we are pressed by applications from foreign countries. It seems to me clear that we must in the first place endeavor to find work for those whom we have encouraged to train themselves in this country on the theory that, if they were worthy, there would be jobs waiting for them. Until we have done that, what else can we do? Our opportunities for making places for foreigners are therefore at the moment limited to a few outstanding personages such as Einstein and Weyl ...."

For his part, Veblen pressed for a more active stance, not only advocating that the Institute do all it could, but also endorsing the establishment of a formal network to provide assistance to scholars in need. He wrote to Flexner on May 5: "Some kind of a committee to raise funds for the purpose of enabling some of them to live and continue their scholarly work in the countries adjacent to Germany or elsewhere might be feasible. The existence of such a committee would in itself be an eloquent protest." That same month, the Institute of International Education in New York City set up its Emergency Committee in Aid of Displaced German Scholars (later renamed to include all Foreign Scholars), headed by Edward R. Murrow, to assist scholars fleeing Europe. Veblen would join its board soon thereafter.

Over the course of the ensuing years, Flexner would undergo a conversion, becoming more deeply involved in assistance projects-he followed Veblen into the Emergency Committee-and increasingly willing to mobilize the Institute to this effect. In a 1938 letter to George Birkhoff at Harvard, he insisted that national origin should never stand in the way of higher goals: "Let us keep firmly in front of our eyes our real goal, namely the development of mathematics, not American mathematics or any other specific brand of mathematics, just simply mathematics. It can be developed only by having first-rate men in important posts, and every time an institution gets one first-rate man he creates opportunities for other first-rate men, and every such center that is developed stimulates some other institution to do likewise. Hitler has played into our hands and is still doing it like the mad man that he is. I am sorry for Germany. I am glad for the United States. I will undertake to get a position within a reasonable time for any really first-rate American mathematician, and I will also undertake simultaneously to do the same for any 
first-rate foreign mathematician whom Hitler may dismiss. The more the merrier."

In his director's report the following spring, Flexner even cast the matter as heralding a seismic change in the geography of knowledge: "We are living in an epoch-making time. The center of human culture is being shifted under our very eyes. Once it had its home in Athens. A few centuries later it had its home in Italy, a few centuries later in Paris, and thereafter also in Great Britain and Germany. It is now being unmistakably shifted to the United States. The scholars of Europe are refugees driven out of their own countries sometimes for political or religious reasons and sometimes because they are too unhappy and too distracted to pursue the work to which they are giving their lives. They have come to the Institute or have corresponded with the Institute literally by the hundreds. We cannot, of course, undertake either to give them places or to find them places, though we have done something substantial under both heads. Fifty years from now the historian looking backward will, if we act with courage and imagination, report that during our time the center of gravity in scholarship moved across the Atlantic Ocean to the United States. It is a grave responsibility which is thus being thrust upon us all." From a vantage point almost eighty years later, Flexner's claim seems more prophetic than hyperbolic. Although the transference in scientific work away from German and toward global English may have begun earlier, the center of gravity clearly shifted in the second half of the twentieth century. Germany's leading share of Nobel Prizes plummeted after the war, even as the number of American laureates soared (one third of whom were foreign born).

\section{"A wall of bureaucratic measures"}

The actual process of reaching the United States was far from simple for most would-be immigrants, who had to navigate not just an ocean, but also a maze of paperwork to obtain the requisite permission to exit and enter. To assist them, Flexner and Aydelotte used their extensive contacts and pulled strings as much as possible. When the mathematical logician Kurt Gödel found himself unable to leave Vienna in October 1939, Flexner contacted the chief of the visa division at the Department of State to plead on his behalf. Although Gödel had been legally admitted as a permanent resident earlier in the 1930s, he had returned to the annexed country that used to be Austria, and was facing difficulty getting authorization to return to the United States. "Is there anything that the State Department or the Consul General can do," Flexner asked, "to suggest some helpful method of procedure?" The American authorities answered that the problem seemed to be with German authorities, and so Flexner's successor Aydelotte contacted the German embassy in Washington. Eventually Gödel and his wife Adele were permitted to leave. By German directive they traveled east instead of west, avoiding
British surveillance of the Atlantic by crossing Siberia and eventually getting to Japan in 1940, where they found a boat to San Francisco. In a letter thanking Aydelotte (Figure 2), Gödel mentioned, "I am told in all steamship bureaux that the danger for German citizens to be arrested by the English is very great on the Atlantic."

Even after refugees succeeded in reaching the United States, they needed to stay bureaucratically alert, and often required assistance. Under the Alien Registration Act of 1940, the US Immigration and Naturalization Service collected fingerprints and required noncitizens to record all changes of address (see Figure 3). Even local travel could necessitate permission, such that Gödel, once finally settled in Princeton, had to request permission to travel with his wife to visit a doctor in New York City in January 1942. They always went by train, Gödel assured the US Attorney, and returned on the same day. Three weeks later, Aydelotte's secretary sent a follow-up plea, noting, "If you could grant them this permission promptly it would be a great relief to them and would be very much appreciated."

The Institute's most famous scholar in exile, Albert Einstein, underscored the unending hassles that hindered attempts to welcome foreign scholars in a letter he wrote

$$
\begin{aligned}
& \text { Vimmar Jan. 5, } 1940 \\
& \text { Dear D Agole (The } \\
& \text { I am greatly obeiged to yow for the request, which you } \\
& \text { have addrenced on my behalf to the Germain chargé } \\
& \text { ol' Affoines in Washingtow. IT is probably on this account } \\
& \text { Thut I was ultimatey yranted leave by the Germoin } \\
& \text { unthrities. In viev of Plop. Veflens Cethe of Dec. } 1 \\
& \text { I un hoping mor, that it will make no difficulty } \\
& \text { to obtaim the Amaiarn vise and that } J \text { shall be able } \\
& \text { to leave Viomse in a fer dags. So the only compliation } \\
& \text { which remains is, that I shall have to Take the route } \\
& \text { though Russin and Japan. The Germain certificate of } \\
& \text { lever makes explicitly this reguinemant and in adolition } \\
& \text { I om told in all STeamsip. buscanx, that the danyer } \\
& \text { in Germain citizens to be anested by the English } \\
& \text { is very greut on the ATlantic. } \\
& \text { I ugnet very much, that the tip over Jupan }
\end{aligned}
$$

Figure 2: Letter from Kurt Gödel to IAS Director Frank Aydelotte, January 5, 1940, expressing gratitude for his and Flexner's help in securing permission for the Gödels to leave Vienna. 


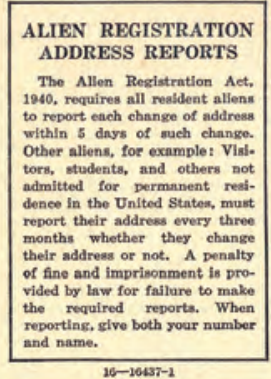

DEPARTMENT OF JUSTICE, IMMIGRATION AND NATURALIZATION SERVICE, ALIEN REGISTRATION DIVISION, PHILADELPHIA, PA.

Figure 3: Under the Alien Registration Act of 1940, noncitizens were required to record all changes of address, and even local travel could necessitate permission.

to Eleanor Roosevelt on July 26, 1941: "A policy is now being pursued in the State Department which makes it all but impossible to give refuge in America to many worthy persons who are the victims of Fascist cruelty in Europe. Of course, this is not openly avowed by those responsible for it. The method which is being used, however, is to make immigration impossible by erecting a wall of bureaucratic measures alleged to be necessary to protect America against subversive, dangerous elements."

\section{A Call for Vigilance}

As we immersed ourselves in the thicket of correspondence at the heart of the Institute's archives, the sense of urgency expressed by scholars like Flexner, Veblen, Aydelotte, Einstein, and Noether resonated deeply. Their notes and exchanges, not to mention the Emergency Committee in Aid for Displaced Foreign Scholars that Flexner and Veblen served on, had an unsettling contemporary ring to them. This part of courage to provide sanctuary for displaced refugees the Institute's history testifies to the individual courage of these men and women who extended a helping hand and built institutional networks to provide sanctuary for displaced refugees. In doing so, they overcame the nationalist siege-mentality that sees foreigners, whether they are mathematicians or fruit pickers, as a threat to be warded off. An unintended consequence of their acts was the shifting of the center of intellectual research from Germany to the United States, enriching the country that gave them refuge. Their individual initiatives and collective institution-building endeavors provide us with much-needed exemplars of moral fortitude.

There is a darker side to this story. It only took a few months and one edict purging civil servants of nonAryan descent or exhibiting suspect political sentiments in April 1933 to drain the German university of many of its brightest minds and its intellectual vigor. Of course, the contemporary political situation in the United States remains far from this extreme case. Nonetheless, knowledge of this history should serve as a call for vigilance in the face of policies such as travel bans and immigrant deportations, as well as attempts to curb scientific inquiry and cut funding to arts and humanities endowments that now threaten the autonomy of research and the pursuit of a dignified human life. Unfortunately, history suggests it takes much less time to destroy than to build. As it did in the 1930s, the Institute can play a leading symbolic role in our contemporary predicament.

\section{Emmy Noether}

To Einstein, she was "the most significant creative mathematical genius thus far produced since the higher education of women began." More straightforward in his praise, Einstein's fellow professor at the Institute for Advanced Study, Weyl, called her a "great woman mathematician [...indeed] the greatest that history has known." It was April 1935, and Einstein and Weyl were each paying tribute to a recently deceased colleague who had, like them, fled Nazi persecution across the Atlantic only two years earlier. Her name was Emmy Noether, and her short but remarkable life left an indelible
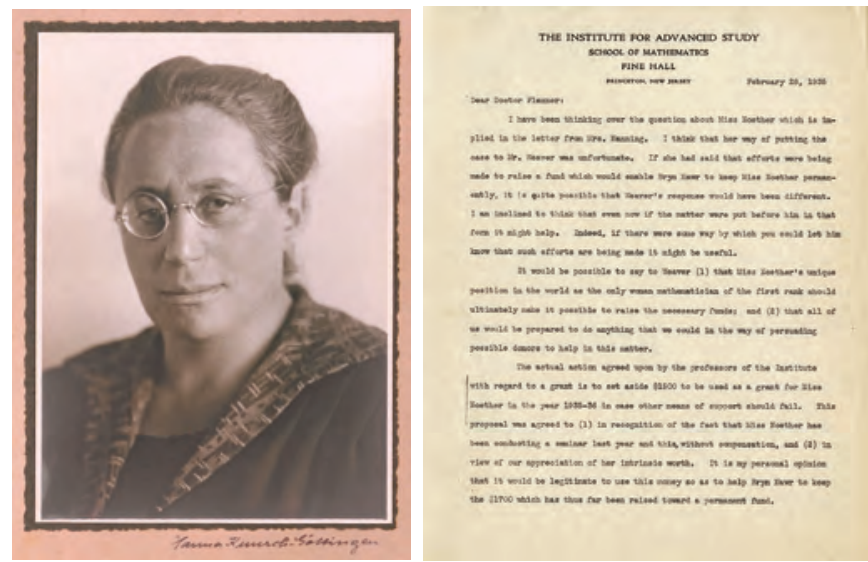

Figure 4: Left: Mathematician Emmy Noether, who had been forced to flee Göttingen, began giving weekly lectures at IAS as a Visitor in the School of Mathematics. Right: Letter from Oswald Veblen to Abraham Flexner, dated February 28, 1935, regarding a $\$ 1,500$ grant for Noether.

mark not only on the history of mathematics, but also on that of IAS in its critical first years.

Amalie Emmy Noether was born in 1882 into an affluent family from the Bavarian town of Erlangen. She followed her father's footsteps to study mathematics at the University of Erlangen and, in 1907, she became the second woman to obtain a PhD in mathematics from a German university. A female maverick in a man's world, Noether taught for several years without pay before being invited, in 1915, to join the University of Göttingen, home to the most prestigious mathematics department in the world at 
the time. She lectured for other professors and was only allowed to pass her habilitation following the collapse of the Kaiserreich and sweeping university reforms in 1919. Noether became an adjunct professor in 1922-the first female professor in Germany-but only started receiving a modest compensation for her teaching the following year.

Despite international recognition, she never obtained a permanent position in Göttingen, and her situation took a turn for the worst with the rise to power of the Nazi party. In 1932, she was denounced by a neighbor as a "Marxist Jewess" and had to leave her apartment. The following year, she was removed from all teaching duties at the university and was eventually forced to flee Germany like many other purged academics. (See Figure 4, left.) Thanks to the intervention of the Emergency Committee in Aid of Displaced German Scholars, Noether was able to take a temporary position at Bryn Mawr College. Once in Pennsylvania, she reconnected with her former Göttingen colleague Weyl, himself freshly recruited to the Institute for Advanced Study by its first director, Flexner, and resident professors Veblen and Einstein.

It was during her years in Göttingen that Emmy Noether developed an international reputation as a formidable mathematician. She made seminal contributions to the field of abstract algebra, where she identified a simple, yet elegant, property of number systems, which proved instrumental in the study of arithmetic and geometric phenomena such as prime decomposition and dimension. (See the sidebar on Emmy Noether's contributions to algebra.)

Noether brought similar clarity to her pioneering research in physics, where she understood the relationship between symmetries of the laws of nature and the notion of "conservation laws." As an illustration, consider the "principle of energy conservation," a paradigmatic conservation law, which states that the total energy of an isolated system cannot change. When a car accelerates, for instance, its energy increases, implying that it must have drawn energy from somewhere, according to the principle of energy conservation (in this case, from burning gasoline). On the other hand, consider "time-translation invariance," a fundamental symmetry of the laws of nature, which states that an experiment performed today would give the same outcome if performed tomorrow: the speed of a free-falling cannonball is the same now as it was in the time of Galileo. Noether was able to connect these two seemingly unrelated concepts: energy conservation comes from time-translation invariance and vice versa. Scientists had long known the connection between energy and time, but Noether was the first to theorize a systematic correspondence: symmetries and conservation laws are related, in general. The idea arose out of a debate between David Hilbert, Felix Klein, and Einstein over the notion of energy in Einstein's recently formulated general theory of relativity. Noether's theorem not only laid this controversy to rest, but, due to its striking generality, has been widely used in many other fields of physics, perhaps most notably in the study of elementary particles.

\section{Rethinking the Scholar's Paradise in the 1930s}

Emmy Noether was thus already a household name among mathematicians when Veblen approached Flexner about supporting her in the United States. Their personal correspondence reveals how the Institute was forced to reconsider its mission in the face of unprecedented assaults on scholars in Europe. Noether's position at Bryn Mawr was funded by the Rockefeller Foundation (as part of their $\$ 1.5$ million aid package for displaced scholars) but was only temporary. At Veblen's invitation, she began giving weekly lectures at the Institute as a visitor in the School of Mathematics, where she joined the first cohort of IAS Members. Noether was happy to be at the Institute-and not at Princeton's "men's university, where nothing female is admitted," as she once said-but she didn't receive any honorariums for her lectures, unlike seventeen other occasional visiting lecturers, all male, who spoke at the Institute throughout the 1930s. On the other hand, Veblen did request a small grant-in-aid, to help keep her at Bryn Mawr through 1935 and 1936, on the grounds of "Miss Noether's unique position in the world as the only woman mathematician of the first rank." Flexner was sympathetic to Noether's plight, but worried about the Institute overcommitting, and he repeatedly encouraged Veblen to view the question as an administrator. Flexner wondered what such a short-term commitment could achieve and expressed concern at the Institute doing any more than what it already had for German scholars, as it needed to be "careful not to create the impression that [it was] overlooking Americans in order to help these unfortunate foreigners." Sidestepping the thorny issue of nationality, Veblen was eventually able to secure a $\$ 1,500$ grant and continued soliciting larger donations for a "permanent commitment on the part of the Institute." (See Figure 4, right.) As he put it, Noether was not merely unique as a "woman mathematician," she offered the Institute an opportunity to capitalize on the brain-drain from Göttingen by supporting "one of the most important scientists" displaced by the events in Germany.

Assisting endangered scholars presented, in other words, as many opportunities as it entailed risks and burdens for the newly established IAS. Flexner himself came around to seeing this, albeit only after Noether's untimely death on April 14, 1935. No doubt inspired by Weyl's and Einstein's stirring obituaries, he invoked her memory in a lecture on "The Usefulness of Useless Knowledge"-an idea he had long pursued and would, two years later, publish as his famous article in Harper's magazine-that he gave at Bryn Mawr on June 2, 1937. Noether, he had come to believe, "was driven from Göttingen for no better reason than that she was a Jewess." Bryn Mawr had "welcomed her with open arms," as many other institutions had done for other scholars at risk across the country. "This is civilization," Flexner concluded, "this is culture... . a country like America, of which only a small fraction has been culturally developed, can only be enriched by the folly which drives great thinkers, novelists, dramatists, and poets out of the Old World to make a fresh start in the New." Flexner's words ring loud today, as a reminder of how the Institute 


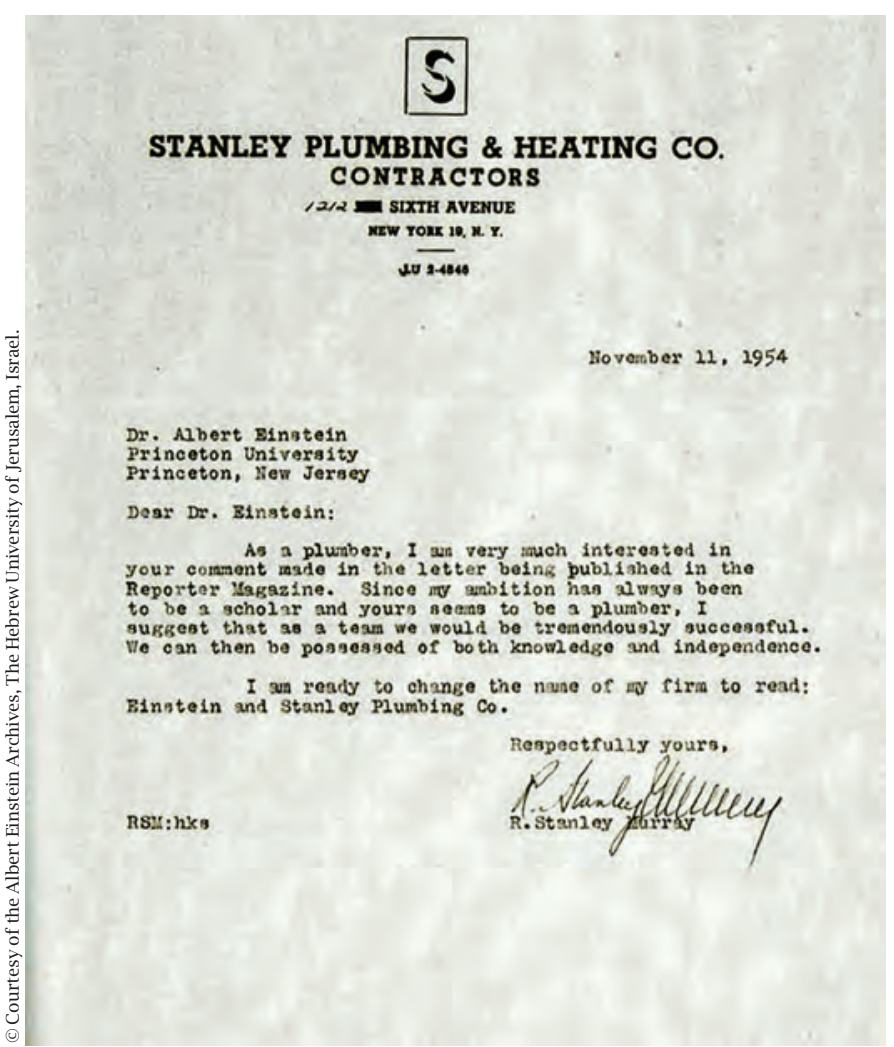

Figure 5: Stanley Murray, a New York City plumber, responded to Albert Einstein's professed interest in being a plumber.

found a new raison d'être in challenging times, and as an admonition of how fragile and contingent "scholars' paradises" such as the IAS, or Göttingen before it, can be.

\section{Einstein, Plumbers, and McCarthyism}

In November 1954, Einstein wrote a letter to a magazine in which he declared that, were he a young man again, he would not try to become a scientist: "I would rather choose to be a plumber or a peddler in the hope to find that modest degree of independence still available under present circumstances." Around the United States, plumbers responded. The famous physicist was offered membership in the Chicago plumbers union, and Stanley Murray, a New York plumber, wrote to him (Figure 5): "Since my ambition has always been to be a scholar and yours seems to be a plumber, I suggest that as a team we would be tremendously successful. We can then be possessed by both knowledge and independence. I am ready to change the name of my firm to read: Einstein and Stanley Plumbing Co."

Einstein was only half joking, however, when he issued his statement. The physicist sincerely considered that the political climate in the country was becoming increasingly hostile to scientists and teachers. Our own troubled times have many aspects in common with the dreadful period of the McCarthy investigations: the attacks on the freedom of academics, teachers, and the press, the silencing and censorship of government workers, the idea that the
United States is threatened by certain creeds. It is worth describing the dire sequence of past events, and learning from Einstein's clairvoyant and courageous response to them, in order to best address the present situation.

On February 9, 1950, Senator Joseph McCarthy from Wisconsin announced that he had a list of 205 workers of the State Department who were members of the Communist Party. The next day, a journalist asked to see the list. But McCarthy could not find it; his explanation was that he had left it in another suit. The Senate committee that was created to investigate these claims concluded a few months later that McCarthy's accusations represented "perhaps the most nefarious campaign of half-truths and untruth in the history of this republic." Historians are now sure there never was such a list.

Nevertheless, at the time, mainstream Republicans ignored the findings of the Senate committee. They saw McCarthy's tactics as something that would help them take control of the White House, after a sixteen-year absence. They invited him to meetings where he ranted about the "plot" at the highest levels of government. McCarthy's staff also circulated a doctored photograph, purportedly showing the leader of the Senate committee in close conversation with leaders of the Communist Party.

The 1952 elections were a great success for the Republicans, who gained control of the White House, the House of Representatives, and the Senate. Senator McCarthy's power was unchecked. During a speech on the Senate floor, he piled hundreds of documents on a table, claiming they contained evidence of the infiltration. No one was permitted to examine them. McCarthy was nominated chair of the Permanent Subcommittee on Investigations, and he extended the loyalty inquisition to many sectors, foremost among them, the nation's educational system. As a former president of the University of Chicago noted, "The entire teaching profession of the US is now intimidated."

William Frauenglass, a teacher in a Brooklyn high school, was called before the Senate subcommittee in April 1953. In his case, the accusation of disloyalty stemmed from a course he had given six years before, in a session for other teachers organized by the Board of Education. It was called Techniques of Intercultural Teaching, and it reviewed methods to "help ease intercultural or interracial tensions" in the classroom. One witness called upon by the committee declared that such teachings were "against the interests of the United States." Frauenglass was shocked: "Imagine such an accusation when one of the fundamental objectives of public education is the creation of intercultural understanding among our many minorities!" he wrote in a later letter. The teacher was also asked which organizations he belonged to, and he refused to answer.

Frauenglass needed help and decided to ask it from someone he greatly respected, who had recently described himself as an "incorrigible nonconformist": Albert Einstein, Professor of Theoretical Physics at the Institute for Advanced Study in Princeton, undoubtedly the most famous scientist in the world, and also a notorious antiracist and antiwar activist. "A statement from you would be most helpful in rallying educators and the public to 
meet this new obscurantist attack," Frauenglass wrote. Einstein obliged. His May 16, 1953, letter of reply-which, he specified, "need not be considered confidential"-was quoted extensively by The New York Times:

The reactionary politicians have managed to instill suspicion of all intellectual efforts into the public by dangling before their eyes a danger from without. Having succeeded so far, they are now proceeding to suppress the freedom of teaching and to deprive of their positions all those who do not prove submissive, i.e., to starve them.

Einstein strongly advised the teacher to refuse to testify any longer. He should be prepared, Einstein wrote, "for the sacrifice of his personal welfare in the interest of the cultural welfare of his country." The physicist added, "This kind of inquisition violates the spirit of the Constitution. If enough people are ready to take this grave step, they will be successful. If not, then the intellectuals of this country deserve nothing better than the slavery which is intended for them." When Frauenglass and Einstein met in Princeton a few days later, Einstein said he himself was ready to go to jail for these principles. Frauenglass followed his advice. As was foreseen, he was fired from his job, but nevertheless thanked the scientist for a "historic letter": "Its echoes are still reverberating throughout the world."

McCarthy was quick to react to Einstein's stand. He told the media that whether his "name is Einstein or John Jones," the giver of such advice was undoubtedly an "enemy of America," "a disloyal American," and "not a good American." But Einstein was in no way deterred. In remarks he made to an assembly of lawyers, he continued to criticize practices "which have "the right to become incomprehensible to publish and teach what
one holds to
be true"
-Einstein the rest of civilized mankind and exposed our country to ridicule." And he warned, "the existence and validity of human rights are not written in the stars."

Einstein was concerned about the curtailing of academic freedom. In a public statement in March 1954, he advocated for "the right to search for truth and to publish and teach what one holds to be true." He regretted that in this dark age "freedom of teaching, mutual exchange of opinions, and freedom of press and other media of communication are encroached upon or obstructed," adding that "this is a state of affairs which a democratic government cannot survive in the long run."

For some, these statements were proof of Einstein's disloyalty and continued foreignness-he the German Jew who had been granted American citizenship in 1940. In March 1954, a woman from Los Angeles wrote to the Director of the Institute for Advanced Study: "The man needs lessons in Americanism. I have no patience with this idea that a person who has performed a great deed or discovered something, should be excused from what citizens of U.S.A. must conform to, or that they need not account for questionable acts of theirs." A man from New York City put it more bluntly: "I suggest he move to Russia-and soon! We don't need him."

The Director of the Institute at the time, Robert Oppenheimer, himself a target of McCarthy's inquisition, remained steadfast in his support of his famous colleague. Six months later, in December 1954, McCarthy was finally "condemned" by a large majority of his Senate colleagues for "contemptuous" and "reprehensible" conduct. Of course, Einstein's actions did not by themselves cause McCarthy's downfall. But they certainly facilitated it, by reaffirming essential principles that date back to the Enlightenment, and by empowering many others to keep up the continuing fight to protect democracy.

\section{Image Credits}

Figure 1 courtesy of HIAS archive.

Figure 2 Director's Office, Faculty Files, Box 13. "Kurt Gödel." From the Shelby White and Leon Levy Archives Center, Institute for Advanced Study, Princeton, NJ, USA.

Figure 3 Director's Office, General Files, Box 67. "U.S. Immigration and Naturalization Service to 1955." From the Shelby White and Leon Levy Archives Center, Institute for Advanced Study, Princeton, NJ, USA.

Figure 4 (right): Director's Office, Faculty Files, Box 32. "Oswald Veblen." From the Shelby White and Leon Levy Archives Center, Institute for Advanced Study, Princeton, NJ, USA.

Figure 4 (left): Photographic portrait of Emmy Noether, Photo Archives, Special Collections Department, Bryn Mawr College Library.

Figure 5 digital image photographed by Mr. Ardon Bar Hama. Photo of Fadi Bardawil by Børre Ludvigsen.

Photo of Thomas Dodman courtesy of Thomas Dodman. Photo of Ian Jauslin courtesy of Ian Jauslin.

Photo of Pascal Marichalar courtesy of Pascal Marichalar. Photo of Klaus Oschema courtesy of Klaus Oschema. Photo of Peter Redfield by Zoë Tomášková Redfield. 


\section{Emmy Noether's Contributions to Algebra}

From the brochure ${ }^{8}$ for the annual Noether Lectures:

Known primarily for her profound and beautiful theorems in ring theory, Emmy Noether's most significant achievement runs deeper: she changed the way mathematicians think about their subject. "She taught us to think in simple, and thus general, terms... homomorphic image, the group or ring with operators, the ideal... and not in complicated algebraic calculations," said her colleague P. S. Alexandroff during a memorial service after her death. In this way, she cleared a path toward the discovery of new algebraic patterns that had previously been obscured.

\section{From the obituary by B. L. van der Waerden: ${ }^{9}$}

Through her study of the arithmetical theory of algebraic functions, Emmy Noether became familiar with Dedekind's theory of modules and ideals. In her 1920 paper (with Schmeidler), she developed and used concepts from the theory of modules: direct sums and intersections, residue class modules, and isomorphisms of modules. These ideas appear like a red thread throughout her later work.

The first major success of her methods was achieved in 1921 in the classic paper "Idealtheorie in Ringbereichen." Following definitions of the terms "ring" and "ideal," she shows that a finiteness condition (the ascending chain condition) is equivalent to Hilbert's theorem of the finite ideal basis.

Her work forms the immovable foundation of today's "general theory of ideals" and has spawned a long series of highly productive efforts.

The module-theoretic concepts from which she had developed her commutative theory of ideals proved their strength in a non-commutative context as well. Emmy Noether established the close connections among the theories of representation, of modules, and of ideals-first in her lectures in 1924 and then clarified and generalized in her lectures of 1927-1928 and in the resulting paper.

\footnotetext{
${ }^{8}$ The Emmy Noether Lectures, About Emmy Noether (2005), written by Allyn Jackson. www. awm-math.org/noetherbrochure/ AboutNoether.htm 1

${ }^{9}$ Excerpted and paraphrased from B. L. van der Waerden's obituary, published in Mathematische Annalen in 1935 and reprinted in Auguste Dicke's Emmy Noether 1882-1935, transl. H. I. Blocher, Birkhauser Boston (1981) pp. 100-106.
}

EDITOR'S NOTE. See also the following related Notices articles:

Noether's Legacy: Rings in Geometry, by Karen Smith (Jan. 2016) www.ams .org/notices/201601/rnoti-p7.pdf

Mathematicians Fleeing from Nazi Germany: Review of the book by the same title by Reinhard Siegmund-Schultze; Reviewed by Michèle Audin (Nov. 2010) www. ams . org/notices/201010/rtx101001300p.pdf

Emmy Noether: The Mother of Modern Algebra: Review of the book by the same title by M.B.W. Tent; Reviewed by Benno Artmann (Oct. 2009) WWW.ams.org/notices/200909/rtx090901105p.pdf

In Puris Naturabilus: Review of the book "Pursuit of Genius: Flexner, Einstein, and the Early Faculty at the Institute for Advanced Study" by Steve Batterson; Reviewed by Lewis Pyenson (Aug. 2008) www. ams . org/ notices/200807/tx080700793p.pdf

The Vision, Insight, and Influence of Oswald Veblen, by Steve Batterson (May 2007) www.ams.org/notices/200705/fea-batterson-web.pdf

The IAS School of Mathematics at 75, by Susan Friedlander and Mark Goresky (Sept. 2005)www. ams .org/ notices/200508/comm-ias.pdf

The IAS School of Mathematics, by Allyn Jackson (Sept. 2002) www . ams .org/notices/200208/fea-ias .pdf 


\section{COMMUNICATION}

\section{ABOUT THE AUTHORS}

Fadi Bardawil investigates the traditions of intellectual inquiry, practices of public criticism, and modalities of political engagement of contemporary Arab intellectuals, both at home and in the diaspora. In doing so, he tracks the international circulation of theoretical discourses. His current project examines the high tides and ebbing away of leftist revolutionary thought and practice in the Levant.

Thomas Dodman is writing a microhistory of a young soldier and his adoptive family in the age of the French Revolution (1790s-1820s). His study draws on a unique collection of letters and diaries to explore the diffusion of Enlightenment ideas, the impact of war, and a family's emotional life through an era of unprecedented social and political transformation.

Ian Jauslin's research focuses on the mathematical aspects of statistical mechanics and solid state physics. In particular, he is interested in phase transitions in classical and quantum many-particle systems, and in rigorous implementations of the renormalization group.

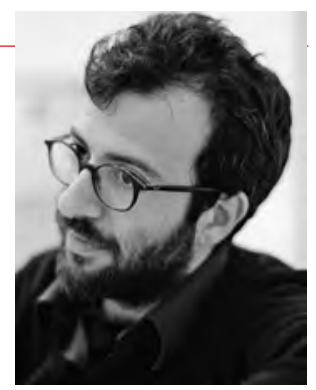

Fadi Bardawil

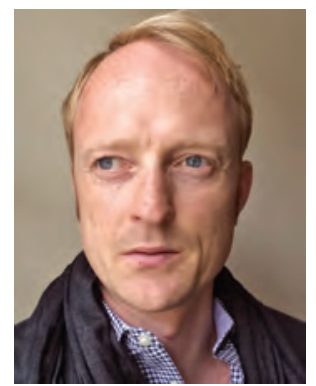

Thomas Dodman

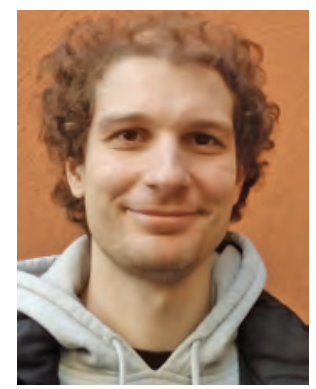

Ian Jauslin
Pascal Marichalar's work is in sociology and history. His research focuses on work, health, pollution and justice.

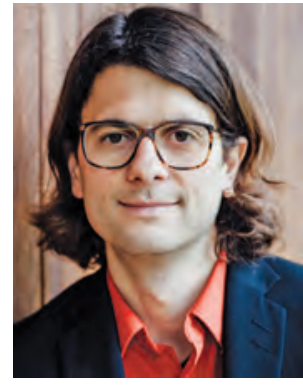

Pascal Marichalar

Klaus Oschema works on astrologers as "experts" in late medieval Europe. He analyzes the mechanisms and strategies, including the aspect of performance, which allowed astrologers to position themselves as experts and to occupy a central role in a variety of contexts of political and social decision-making.

Peter Redfield is exploring humanitarian design and efforts to create innovative devices in response to disaster and poverty globally. His research focuses on conceptions of human needs and ethical responsibilities. He is interested in the politics of technology, particularly questions of scale and accountability beyond state-administered infrastructure.

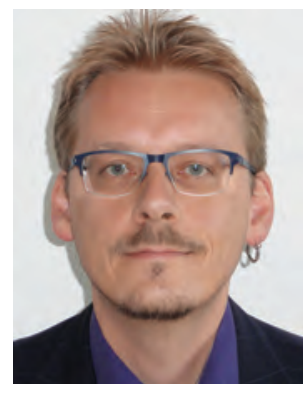

Klaus Oschema

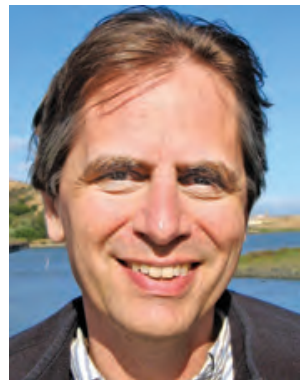

Peter Redfield 


\section{AMERICAN MATHEMATICAL SOCIETY}

\section{Youn donation to the

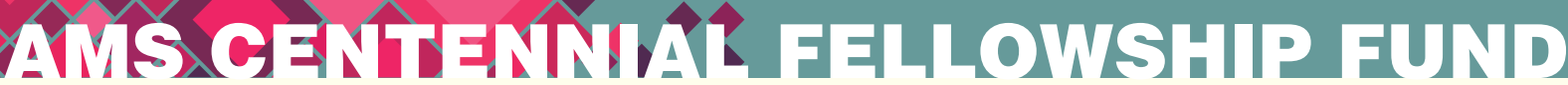 advances mathematics for many.}

Recipients of this one-year mathematics research fellowship have contributed substantially to their areas of research, furthering the mathematical sciences.

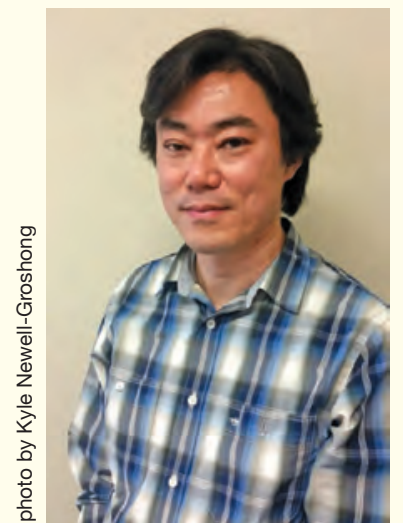

2017-2018 Centennial Fellowship recipient Shuichiro Takeda Associate Professor of Mathematics at University of Missouri. Research interests include automorphic forms and representations of $p$-adic groups, especially from the point of view of the Langlands program
Since 1974, _ scholars have been able to further their area of research with help from the Centennial Fellowship (formerly named Research Fellowship).
a. 43
b. 50
c. 75
d. 106

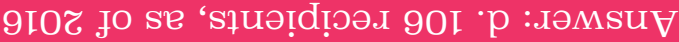

To donate to the Centennial Fellowship,

visit www.ams.org/support

Contact the AMS Development Office by phone: 401-455-4111 or email: development@ams.org 


\section{THE GRADUATE STUDENT SECTION}

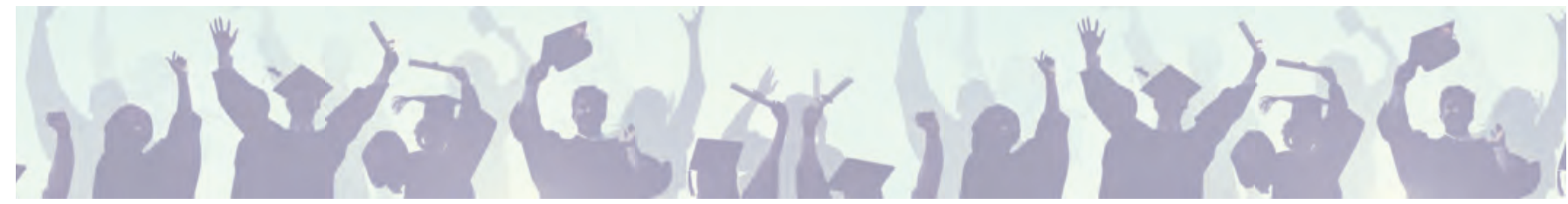

\section{Lillian Pierce Interview}

\section{Conducted by Laure Flapan}

\section{Communicated by Alexander Diaz-Lopez}

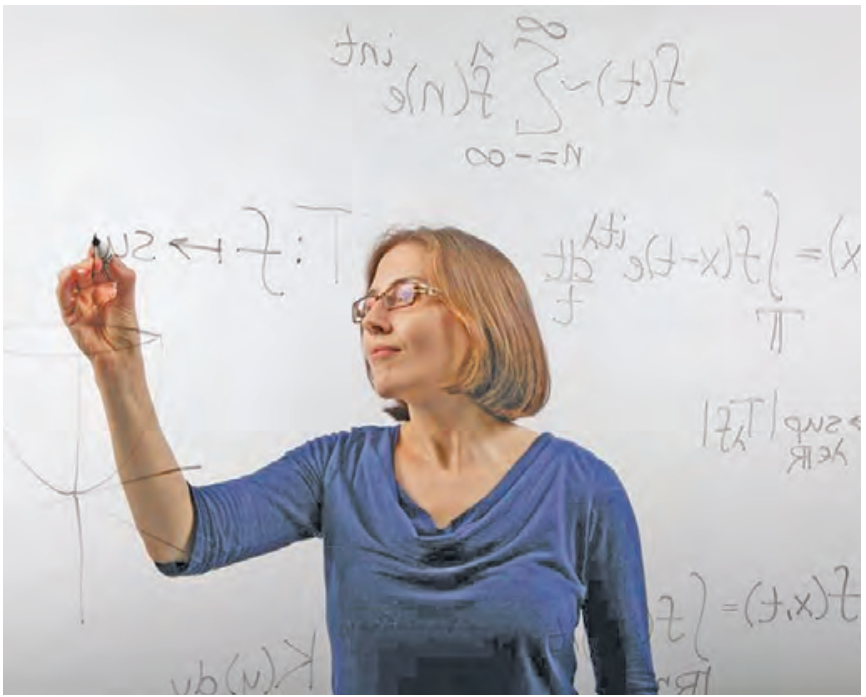

Lillian Pierce is assistant professor of mathematics at Duke University. Her areas of research are analytic number theory and harmonic analysis. She received a CAREER Award from the National Science Foundation in 2017 and a 2017-2018 von Neumann Fellowship from the Institute for Advanced Study in Princeton.
For permission to reprint this article, please contact: reprint-permission@ams.org.

DOI: http://dx.doi.org/10.1090/noti1586
Flapan: When did you know you wanted to be a mathematician?

Pierce: First I had to learn that mathematicians still existed. I was really little when I fell in love with numbers: the way they looked, the way they talked to each other-which is how I perceived arithmetic. I remember a visceral delight in filling out math workbooks in kindergarten. But I don't think I explicitly realized that doing mathematics research was a career until partway through college. I started out as a math major at Princeton because I had "always liked math," but my goal was to head for $\mathrm{MD} / \mathrm{PhD}$ programs, and I completed all the pre-med requirements. Meanwhile, I became absolutely captivated by the feeling of doing mathematics, and this coincided with a stellar sequence of courses at Princeton (which became the series Princeton Lectures in Analysis by Stein and Shakarchi). By the last year of college, my goals became centered on mathematics.

Flapan: Who encouraged or inspired you?

Pierce: I have been encouraged and inspired by dozens of mathematicians, and also more broadly, scientists. I feel extremely lucky in this way. Just now, I started to write down names but the list is so long that it looked like bragging. Many, many people have been generous with their interest in me, generous with suggesting problems or questions to me, generous with checking in on me as I reached a transition point in my education or career, generous with writing me letters of reference or nominations, or inviting me to visit, or to speak at a conference. Of course, even in such a list, my graduate and postdoc mentors stand out: Roger Heath-Brown, Peter Sarnak, Eli Stein. They have each been absolutely foundational in guiding my research interests.

Flapan: How would you describe your research to a graduate student?

Pierce: My work lies in the union of analytic number theory and harmonic analysis. In number theory, I'm interested in counting integral points on varieties via the circle method and sieve methods, and in studying properties of class numbers of number fields. In analysis, I'm interested in bounding oscillatory integral operators, 
Radon transforms, and Carleson operators, and their discrete analogues. In either setting, the methods often hinge upon finding a good bound for an oscillatory integral or an exponential or character sum-this is because many of the methods I use are based on Fourier analysis. I'd say that typically the arguments I like are sequences of simple ideas using recognizable objects. In culinary terms, I'd be more in the philosophy of farm-to-table than molecular gastronomy.

Flapan: What theorem are you most proud of and what was the most important idea that led to this breakthrough?

Pierce: I think it's a bit early to say I'm proud of any one particular theorem. What I am glad of is that I've been able to shift my focus repeatedly in order to work on problems that I find really appealing, even if they aren't obviously related. I get asked "Are you an analyst?" "Are you a number theorist?" I don't really want to make that division, for myself. Nevertheless, sometimes I watch someone give a talk and feel a bit envious that the territory they have chosen to work in consistently for a number of years is so well-defined: Problem A and variations. That person knows everything about the history of Problem A, all the current gossip about Problem A, and can be forgiven for ignoring things on the arXiv that aren't in the neighborhood of Problem A. I on the other hand feel like I've started over building expertise from scratch a number of times. It certainly isn't efficient. But some problems just feel like the right ones.

Flapan: You received your PhD only eight years ago, but you have given over 100 talks. What is your favorite part about giving talks?

Pierce: I learn a lot from giving talks. First, I think afresh while preparing a talk: Why would other people care about the problem? What are the historical origins? What are the connections to other areas? What are the truly key ideas in the proof? How can I strip them of technicalities so that people can remember them intuitively? Second, I learn from people's reactions to the talk. Sometimes I pick up historical facts I didn't know. Sometimes I learn how I could frame the result so that people in another field might find it more familiar. Sometimes I am prompted to strengthen the result or extend the method or start a new project. Third, I really like getting to meet in person the mathematicians I see represented on paper.

Flapan: What are the biggest challenges young mathematicians today face?

Pierce: I wonder about the speed of research now. I haven't experienced other eras, but nowadays it could feel like you are in a crowded international race twentyfour hours a day. When I was deciding on a career, one of the big attractions I felt to math was the contemplative nature of the work. I want to continue to choose to be a contemplative thinker, to write papers with great attention to exposition, to not feel rushed. I have learned a lot about patience from seeing my father, a woodworker, make furniture. Once, he wanted to achieve a certain color for an oak bed, which historically would have been achieved with ammonia fuming. Instead, he devised a non-toxic water-based dyeing strategy, but while this attained the right color, it would have raised the grain and made the wood feel rough. To pre-empt this, he soaked every piece of the bed in water, which raised the grain, and then sanded it down by hand. He did this seven times, at which point the grain was recessed. Then he applied the water-based dyeing strategy, which now raised the grain just flush with the wood, leaving each surface of the bed satin smooth. Every piece he makes is gorgeous, strong, and clever-and comes with a lot of sanding.

Flapan: All mathematicians feel discouraged occasionally. How do you deal with discouragement?

Pierce: Discouragement was a bigger factor in grad school when I had just a couple distinct goals. Now I typically have more projects going, and usually one of them is looking somewhat tractable. Working with collaborators is really helpful-if they are a good mathematical and personal fit, they can really keep your spirits up. In general, I think a time of discouragement can be a time to rephrase what we do as "learning" rather than "proving." After all, proving a new theorem is just learning something no one has learned before. And we probably can each climb back to enjoying math if we temporarily set our sights on just learning something.

Flapan: In a previous life, you were a professional violinist. What role does music play in your life today?

Pierce: Like many people, I listen to music to give me energy as I work, particularly when writing. The Prokofiev piano concerti have been some of my best work music, for years. In another vein, for me, learning to play a piece of music is like building a detailed mental landscape, and that can be very much like assembling the pieces of a longterm math project. There's another thing too-after you have performed a concerto with an orchestra in a large concert hall, giving a math talk seems relatively low pressure. You're allowed to pause and think, for one thing!

Flapan: What advice do you have for current graduate students in math?

Pierce: If I were talking to me as a student, I'd say: "Pick a book and sit down every day and spend one hour working your way through it in detail." In grad school, it can feel like any time not spent banging your head directly on your thesis problem is wasted. But in reality, if you consistently take one hour a day away from that struggle and work through books, you'll gradually gain technical skills that will come in handy one day.

Flapan: If you could recommend one book to graduate students, what would it be? 
Pierce: Find a book in your field that is considered to be especially beautifully written. For me, say, Stein's Harmonic Analysis. Then figure out why it is so good. Math will be more fun for everyone, especially young people, if we all learn to write clearly and generously.

Flapan: Any final comments or advice?

Pierce: Here's a standard set by Anthony Trollope that I one day hope to achieve: "A small daily task if it really be daily, will beat the labors of a spasmodic Hercules."

\section{Photo Credits}

Photo of Lillian Pierce courtesy of Duke Photography.

Photo of Laure Flapan courtesy of Simons Center for Geometry and Physics.

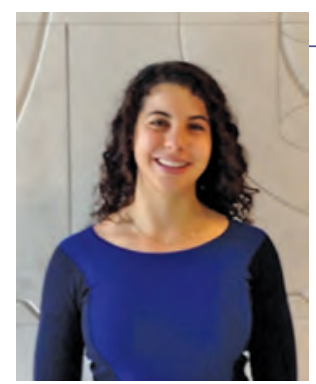

ABOUT THE INTERVIEWER

Laure Flapan is a math $\mathrm{PhD}$ student at the University of California, Los Angeles. Her work is in algebraic geometry, particularly Hodge theory. Her email is $1 \mathrm{flapan@math.uc1a.}$ edu.

Laure Flapan

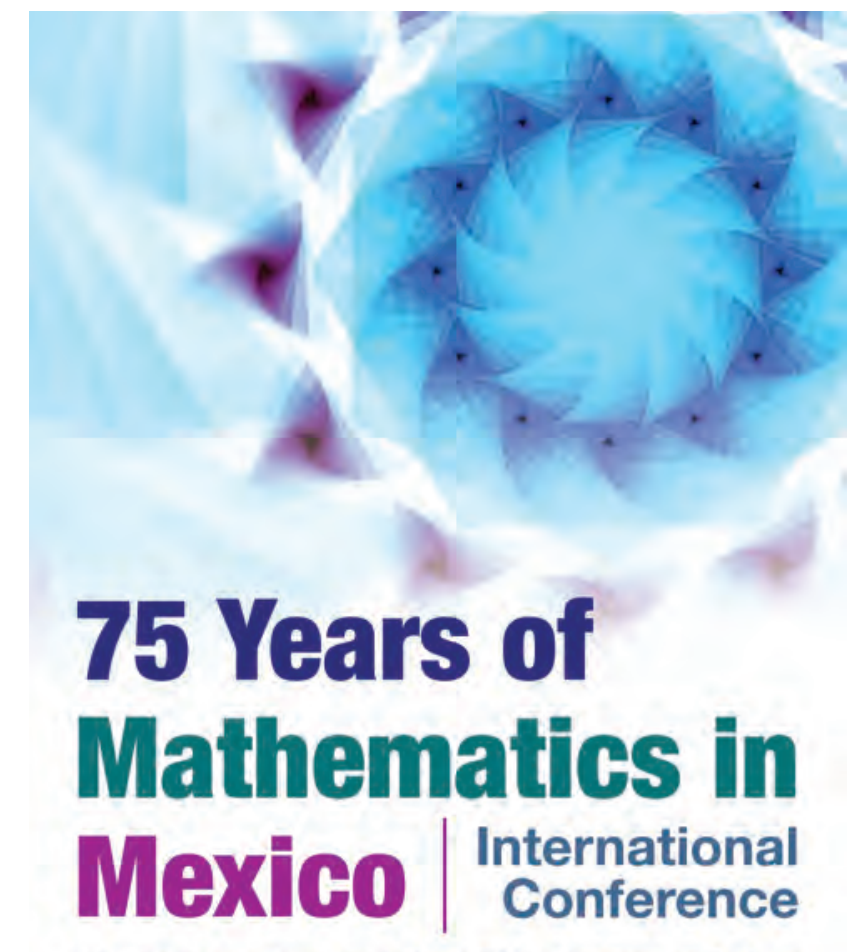

Celebration of the 75 years of the Instituto de Matemáticas, UNAM

December 4-7, 2017 Ciudad Universitaria, Mexico City

Invited Speakers

Noga Alon (Tel Aviv University, Israel)

Luis Caffarelli (University of Texas at Austin, USA)

Mónica Clapp (IM-UNAM, Mexico)

José Antonio de la Peña (IM-UNAM, Mexico)

David Gabai (University of Princeton, USA)

Christoff Geiss (IM-UNAM, Mexico)

Adolfo Guillot (IM-UNAM, Mexico)

Mike Hopkins (University of Harvard, USA)

Isabel Hubard (IM-UNAM, Mexico)

June Huh (University of Princeton, USA)

Kristin Lauter (Microsoft Research, USA)

Pierre Louis Lions (Collège de France)

Dusa McDuff (Barnard College, Columbia University, USA)

Sylvie Méléard (École Polytechnique, France)

John Milnor (University of New York at Stonybrook)

Shigefumi Mori (RIMS, Kyoto, Japan)

Nizar Touzi (École Polytechnique, France)

Gerónimo Uribe (IM-UNAM, Mexico)

Alberto Verjovsky (IM-UNAM, Mexico)

Efim Zelmanov (Univ. Cal. at San Diego, USA)

Distinguished Guest

Jacob Palis (IMPA, Brazil)

75years-im.matem.unam.mx 


\section{THE GRADUATE STUDENT SECTION}

\section{WHAT IS...}

\section{a Varifold? \\ The Editors}

A varifold is one definition of a generalized $m$-dimensional surface in say $R^{n}$. The full class of varifolds is a very general class of measures. To encode information about both position and tangent direction, a varifold is defined as a measure on the cross product of $R^{n}$ with the space of possible tangent planes.

To understand how to view an $m$-dimensional surface in $R^{n}$ as a measure, you need to know that there is a nice $m$-dimensional "Hausdorff" measure $H^{m}$ on $R^{n}$. It assigns to every smooth $m$-dimensional submanifold its standard $m$-dimensional area, but it is defined on a much wider class of sets, including of course all closed sets. Given a nice $m$-dimensional surface $S$, consider the associated measure

$$
\mu_{S}(A)=H^{m}(S \cap A) .
$$

By definition the surface $S$ determines the measure $\mu_{S}$, but it also goes the other way: the measure $\mu_{S}$ determines the nice surface $S$, at least up to sets of measure 0 .

To directly encode information about position and tangent plane, a varifold is defined as a measure on the Cartesian product of $R^{n}$ with the set of all possible tangent planes. For an integral varifold, the tangent planes are coordinated with the surface. In a general varifold, the tangent planes are independent. Such a varifold can model an infinitesimally corrugated diagonal slice of a crystal which favors horizontal and vertical directions, having both horizontal and vertical tangent planes, each with probability one-half, as in Figure 1.

As a space of measures, the space of varifolds has a nice topology and nice compactness properties. Most important are the integral varifolds, which correspond to smooth submanifolds and infinite sums of such. There's also a deep compactness theorem for integral varifolds, but it needs a bound not only on total measure but also on curvature and boundary, to avoid nonintegral varifolds

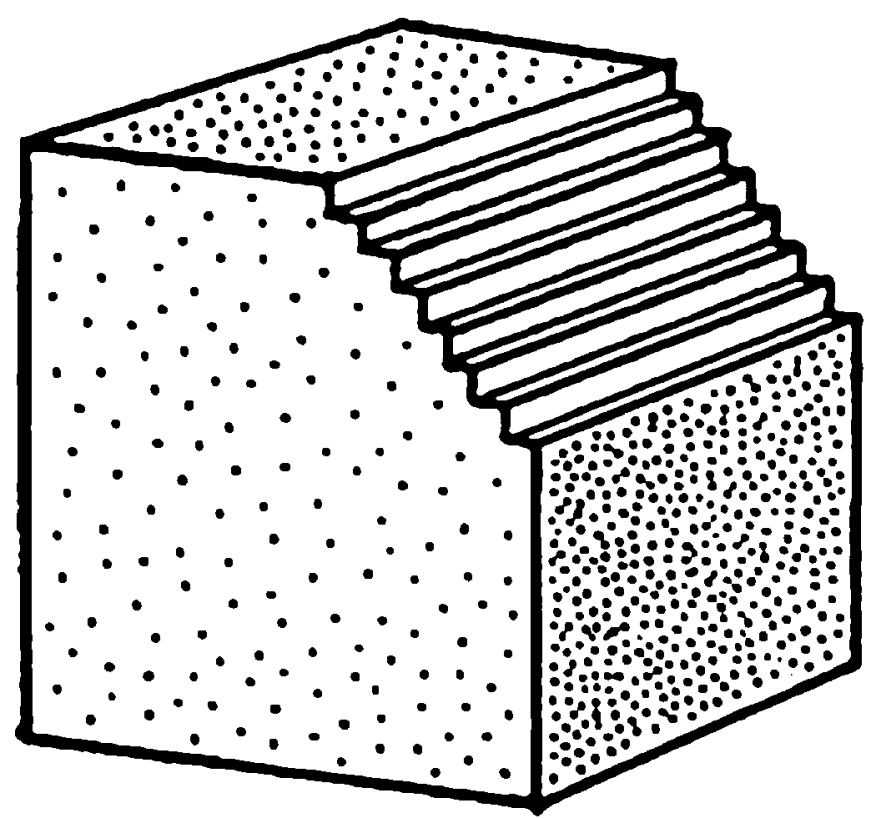

Figure 1. If you cut an edge off a cubical crystal, the exposed surface forms tiny steps, well modeled by a varifold.

in the limit, perhaps as in Figure 1, where the encoded tangent planes are not the geometric ones.

For more about varifolds and current applications, see "The Concept of a Varifold" in this issue of Notices (page 1148).

\section{Photo Credit}

Figure 1 drawn by J. Bredt, from F. Morgan's Geometric Measure Theory, courtesy of Frank Morgan. 


\section{THE GRADUATE STUDENT SECTION}

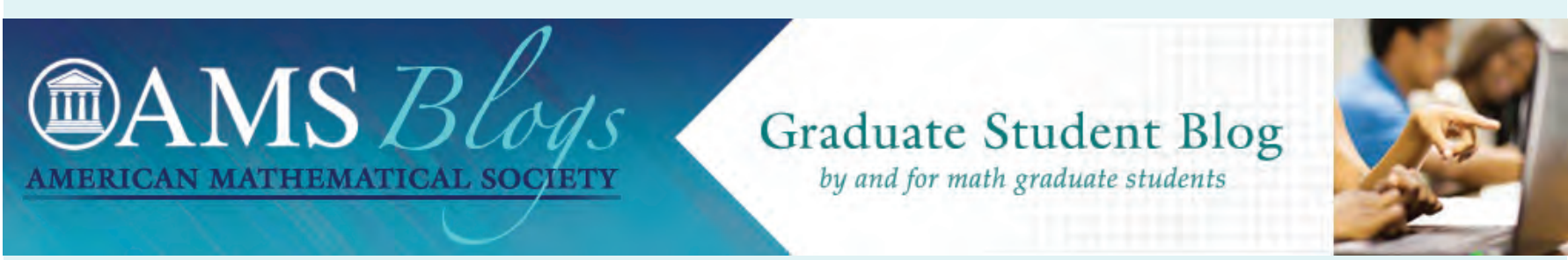

The AMS Graduate Student Blog, by and for math graduate students, includes puzzles and a variety of interesting columns, such as these two from June/July 2017. blogs . ams .org/mathgradblog.

\section{Gauge Theory and Low-Dimensional Topology (Part I: Historical Context) by Irving Dai, Princeton University}

Hi! This month, I thought I would start a brief series of articles describing the uses of gauge theory in mathematics. Rather than discuss current research directions in gauge theory (of which there are many), I hope to give an overview of the sorts of mathematical questions that gauge theory was first used to answer and a general idea of what it is all about. Our goal for today will be to contextualize the initial advances in low-dimensional topology due to gauge theory by giving a picture of the state of affairs before its introduction. We will thus spend this post establishing some basic terms and ideas for the uninitiated; we will wait until later posts to discuss gauge theory itself and what it can tell us about topology.

I have attempted to make this article as introductory and motivational as possible, especially to readers who are less familiar with the finer historical developments in low-dimensional topology. No background is needed except for (at best) a passing recollection of basic algebraic and differential topology. In several places I have been a bit cavalier with precise definitions for the sake of the exposition. All errors are (of course) mine!

\section{In the Beginning, There Were Four-Manifolds}

In order to understand the development of (mathematical) gauge theory, we will first need to know a bit about the history of low-dimensional topology. In our case, the relevant history will be the story of four-dimensional manifolds (affectionately called "four-manifolds," for short). If you are less familiar with low-dimensional topology, then for the moment you can think of these objects simply as "spaces," much like the surface of a sphere or a torus, except (of course) in four dimensions. Four-manifolds include things like $S^{4}$ and $T^{4}$, which are the obvious higher-dimensional analogues of the two-sphere $S^{2}$ and the two-torus $T^{2}$, as well as things like the complex projective plane $\mathbb{C} P^{2}$. For those who are familiar with complex or algebraic geometry, slightly fancier constructions include taking complex hypersurfaces in $\mathbb{C} P^{3}$, and so on. It turns out, however, that mathematicians have come up with a tremendous number of different ways to construct four-manifolds beyond these simple cases, the result of which has been a veritable zoo of examples, each with its own properties and its own "flavor" of construction.

In any first course in topology, one is usually exposed to algebraic invariants such as the fundamental group and/or homology and cohomology. The basic idea behind these is to associate to a topological space an algebraic object-for example, a group (in the case of the fundamental group) or a sequence of abelian groups or vector spaces (in the case of homology or cohomology). One then asks precisely what topological information about a space these algebraic invariants capture. Indeed, one might even hope to construct a complete invariant-that is, an invariant capable of distinguishing two spaces whenever they are different. Failing this, one might at least hope to find an invariant which distinguishes spaces in some well-understood subclass of cases, or up to a known number of ambiguities.

It turns out that in four dimensions, the most interesting classical invariant to study is the intersection form. For those with a bit of background in algebraic topology, recall that this is defined as follows. Let $M$ be a closed fourmanifold, which for simplicity we assume to be simplyconnected. We then have a unimodular bilinear pairing on the cohomology $H^{2}(M ; \mathbb{Z})$ given by the cup product:

$$
(\alpha, \beta)=\langle\alpha \smile \beta,[M]\rangle \in \mathbb{Z}
$$

for any two classes $\alpha, \beta \in H^{2}(M ; \mathbb{Z})$. If you are more familiar with de Rham cohomology, then the above is the same as taking the wedge product of two two-forms and integrating over $M$. If you are not at all familiar with algebraic topology, then the idea of the intersection form is (very roughly) as follows: each element of $H^{2}(M ; \mathbb{Z})$ can be represented as (the dual of) an embedded two-dimensional surface $\Sigma$ in $M$. Any pair of two-dimensional surfaces $\Sigma_{1}$ and $\Sigma_{2}$ in $M$ generically intersect in a finite number of points, and counting the number of such points (with the proper sign) defines an integer-valued pairing on $H^{2}(M ; \mathbb{Z})$.

We have thus associated to $M$ an algebraic invariant which takes the form of an integer-valued bilinear pairing. Intuitively, this invariant captures the collection of all twodimensional surfaces floating around inside $M$ (up to the appropriate equivalence relation) and how they intersect each other. (This picture is very imprecise and should be used for motivational purposes only.) We can of course 


\section{THE GRADUATE STUDENT SECTION}

abstract away this topological intuition and simply study the space of all possible such integer pairings up to an appropriate notion of algebraic equivalence; this is identified with the set of all unimodular lattices. These may be thought of as corresponding to symmetric, integer-valued matrices with determinant \pm 1 , where two matrices $A_{1}$ and $A_{2}$ are equivalent if(f) $A_{1}=B^{T} A_{2} B$ for some integer matrix $B$.

For those unfamiliar with intersection forms, we list here some simple examples. As an exercise, you can try to prove that the intersection forms are as I have stated them:

1) $S^{4}$ : This has no second cohomology. Its intersection form is trivial (empty).

2) $S^{2} \times S^{2}$ : This has second cohomology isomorphic to $\mathbb{Z}^{2}$, with generators represented by the surfaces $S^{2} \times$ (point) and (point) $\times S^{2}$. Its intersection form is given by the $2 \times 2$ matrix

$$
\left[\begin{array}{ll}
0 & 1 \\
1 & 0
\end{array}\right]
$$

3) $\mathbb{C} P^{2}$ : (Complex projective space.) This has second cohomology isomorphic to $\mathbb{Z}$, with a generator represented by the hyperplane class. Its intersection form is given by the singleton matrix [1].

4) $\overline{\mathbb{C} P^{2}}$ (Complex projective space with reversed orientation.) This has second cohomology isomorphic to $\mathbb{Z}$, with a generator represented by the hyperplane class. Its intersection form is given by the singleton matrix [-1].

5) (Not so simple!) Let $M$ be a nonsingular degree-four hypersurface in $\mathbb{C} P^{3}$. (That is, $M$ is defined to be the zero locus of a homogenous degree-four polynomial in $\mathbb{C} P^{3}$; such a hypersurface is often called a $K_{3}$ surface.) Then the second cohomology of $M$ has rank twenty-two, and its intersection form is given by $3 H \oplus 2\left(-E_{8}\right)$. Here, $H$ is the matrix of Example 2 and $E_{8}$ is given by

$\left[\begin{array}{llllllll}2 & 1 & 0 & 0 & 0 & 0 & 0 & 0 \\ 1 & 2 & 1 & 0 & 0 & 0 & 0 & 0 \\ 0 & 1 & 2 & 1 & 0 & 0 & 0 & 0 \\ 0 & 0 & 1 & 2 & 1 & 0 & 0 & 0 \\ 0 & 0 & 0 & 1 & 2 & 1 & 0 & 1 \\ 0 & 0 & 0 & 0 & 1 & 2 & 1 & 0 \\ 0 & 0 & 0 & 0 & 0 & 1 & 2 & 0 \\ 0 & 0 & 0 & 0 & 1 & 0 & 0 & 2\end{array}\right]$

\section{Freedom's Theorem}

The amazing thing about the intersection form is that it turns out to be very nearly a complete invariant of (closed, simply-connected) topological four-manifolds. More precisely, in 1982, Michael Freedman proved the following:

Let $Q$ be any unimodular, symmetric bilinear pairing. Then there exists a closed, simply-connected topological four-manifold $M$ whose intersection form is $Q$. Moreover, if $Q$ is even, then $M$ is unique up to homeomorphism. If
$Q$ is odd, then (up to homeomorphism) there are exactly two closed, simply-connected manifolds with intersection form $Q$.

Here, we say that an intersection form $Q$ is even if $Q(x, x)$ is even for all $x$ (or if it is empty), and is odd otherwise. Examples 1, 2, and 5 above are all even, while Examples 3 and 4 are odd.

It is worth pausing for a moment to appreciate this result. So far, we have encountered two distinct categories of objects in our discussion. On one hand, we have topological objects: simply-connected, topological fourmanifolds. On the other, we have algebraic objects: unimodular lattices. At first glance, it might seem that these are two completely different sets of objects; and, indeed, the study of lattices has a long and storied history within number theory entirely independent of any topology (see for example previous posts on this blog about packing problems!). However, Freedman's theorem says that up to a factor of two, these two things are the same-for every possible intersection form, one can produce a corresponding four-manifold, and at most two such fourmanifolds share any given intersection form! Results of this kind which link an algebraic invariant to the topology of a space are (in some sense) the holy grail of topology and are usually extremely hard to come by. For example, the three-dimensional Poincaré conjecture-which states that $S_{3}$ is the only three-manifold with trivial fundamental group-was only proven in 2006 after remaining open for nearly a century. (Indeed, the astute reader may have already realized that Freedman's theorem applied to Example 1 turns out to give a proof of the four-dimensional topological Poincaré conjecture!)

Roughly speaking, this was the state of affairs in fourmanifold theory immediately before the introduction of gauge theory. Freedman's theorem provided a strong correspondence between four-manifolds and their intersection forms, and indeed was a powerful way to indirectly show that two four-manifolds were homeomorphic. However, a crucial distinction remained: this result held in the topological category, with the manifolds constructed in the theorem being topological manifolds and the notion of equivalence being homeomorphism. It was thus a natural question to ask whether a similar correspondence held for smooth four-manifolds (that is, four-manifolds with differentiable structure, up to diffeomorphism).

To the uninitiated, the distinction between smooth and topological manifolds might seem like a technical point. In fact, in three dimensions or less, every topological manifold admits a unique smooth structure-there is no distinction between continuous and smooth topology in dimension three! However, as we shall see, in dimension four the difference between continuous and smooth topology assumes an all-consuming importance. Indeed, the first major application of gauge theory was to show that smooth four-manifolds exhibit a radically different behavior with respect to their intersection forms - the relation between a four-manifold and its intersection form in the smooth category is very different from the tidy correspondence due to Freedman's theorem in the topological 


\section{THE GRADUATE STUDENT SECTION}

category. This opened up a chasm between continuous and smooth topology in four-dimensions that persists to the present day. (Indeed, while the topological fourdimensional Poincaré conjecture was solved by Freedman, the smooth four-dimensional conjecture remains a major open problem!) Next time, we will attempt to describe this difference and explain how gauge theory could possibly have something to say about it.

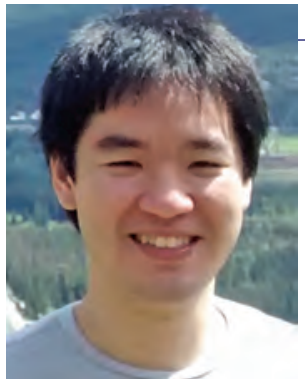

Irving Dai

\section{ABOUT THE AUTHOR}

Irving Dai is a fourth-year graduate student studying topology and geometry at Princeton University. His mathematical interests include gauge theory and related Floer homologies. In his spare time he plays the violin (occasionally, and usually badly). He is fond of cats. His email address is idai@math.princeton. edu.

\section{How to Survive Grad School as a Woman in STEM by Susannah Shoemaker, 1 Princeton University}

There are certain moments from grad school that will always stick with me: the conference in Boston where my usually quiet lab mate opened up to me; the nights I spent drinking cheap beer with my closest friends in the grungy, student-run bar; the time (okay, times) I cried in my advisor's office, ... my general exams, whose residual panic-inducing effects I can still feel, months later.

But I've also had experiences that extend beyond the normal ups and downs. I listened, trying not to cry, as a professor told me that I was too slow to do theoretical work. (A year later, I won an NSF grant to do just that.) I gritted my teeth as I, the only woman in the room, was asked to sort exams into piles, while my male colleagues graded them. These experiences didn't make me stronger, happier, more resilient, or more confident. They just wore away at my well-being.

Learning to survive graduate school as a woman in STEM-or any minority ${ }^{1}$, for that matter-means finding ways to manage the effects of constant, subtle antagonism, because that antagonism won't make you a better scientist, mathematician, or engineer.

Here are seven things that will:

1. Reevaluate your definition of a mathematician... . It took some reexamining of unconscious beliefs before I realized that if I wanted to become a mathematician, I'd have to start thinking of myself as one.

\footnotetext{
${ }^{1}$ See video "Neil Degrasse Tyson on being Black, and Women in Science" at www. youtube.com/watch?v=z7ihNLEDiuM

${ }^{2}$ See "Belief that some fields require 'brilliance' may keep women out" by Rachel Bernstein in Science Magazine atwww. sciencemag. org/news/2015/01/belief-some-fields-require-bri11iance-may-keep-women-out
}

2. Stop using the word "genius."2 ...

3. Start your own alt-boys' club. ...

4. Find an involved advisor...

5. ... and a good therapist. ...

6. Accomplish something. Something outside of your project and coursework, that is....

7. Know why you're in it... . Whatever your career goals are, communicate them-especially if they are unconventional... .

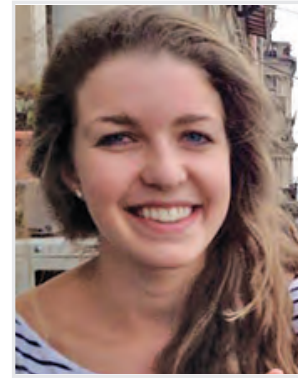

Susannah Shoemaker

\section{ABOUT THE AUTHOR}

Susannah Shoemaker recently earned her MA in applied math from Princeton University. She is interested in the mathematical foundations of structural techniques in biophysical chemistry. Her email address is scs5@princeton. edu.

\section{Photo Credits}

Photo of Irving Dai courtesy of Irving Dai.

Photo of Susannah Shoemaker courtesy of Susannah Shoemaker.

EDITOR'S NOTE. Also, see the helpful post by Jacob Gross on "Some funding opportunities for graduate students in mathematics" blogs.ams.org/mathgradblog/2017/07/19/funding-opportunitiesgraduate-students-mathematics/ 


\section{ROGER \\ PENROSE}

FASHION
Saitfy

FANTAีSY

in the New Physics of the Universe
Fashion, Faith, and Fantasy in the New Physics of the Universe

Roger Penrose

"It is always inspiring to read Penrose's uncompromisingly independent perspective on physics."

-Richard Dawid, Nature

"An extremely original, rich, and thoughtful survey of today's most fashionable attempts to decipher the cosmos on its smallest and largest scales."

-Science

Paper $\$ 17.95$

Cloth $\$ 29.95$

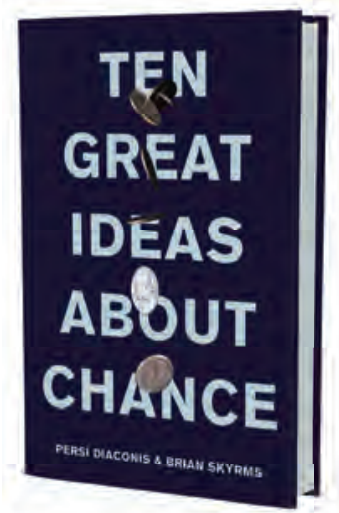

Ten Great Ideas about Chance

Persi Diaconis \& Brian Skyrms

"This compelling book is a welcome contribution to the slim body of recent works that popularize chance. It invites readers to the examples with ease, and it raises far-reaching, thought-provoking questions. I learned a huge amount of useful new information about probability theory and statistics from this book." -Joseph Mazur, author of Fluke:

The Math and Myth of Coincidence Cloth $\$ 27.95$

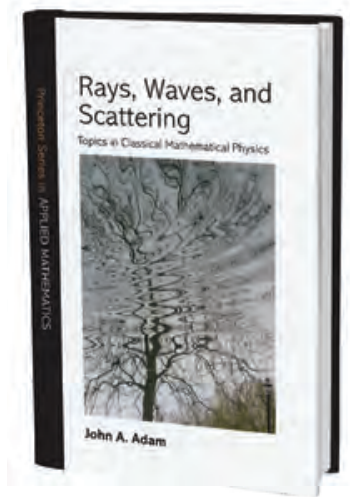

Rays, Waves, and Scattering Topics in Classical Mathematical Physics

John A. Adam

"This is a significant contribution to the literature on wave theory, one that blends the mathematics and physics in just the right way. All derivations are given in full so that the work is genuinely a students' book, and Adam has chosen only the most interesting parts of the subject." —C. J. Chapman, Keele University

Cloth $\$ 85.00$

Princeton Series in Applied Mathematics Ingrid Daubechies, Weinan E, Jan Karel Lenstra, and Endre Süli, Series Editors

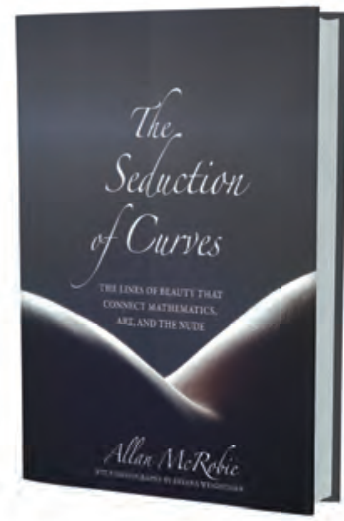

The Seduction of Curves

The Lines of Beauty That Connect Mathematics, Art, and the Nude

Allan McRobie

With photography by Helena Weightman

"This is what a book should be: startling, bold, and beautiful, idiosyncratic and yet universal. Brilliantly conceived, poetically written, and utterly original, The Seduction of Curves is a sinuous delight."

-Steven Strogatz, author of The Joy of $x$ : A Guided Tour of Math, from One to Infinity

Cloth $\$ 35.00$

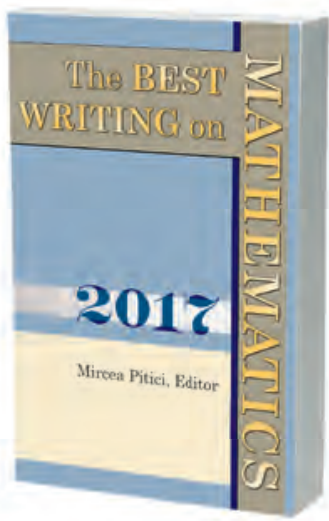

\section{The Best Writing on} Mathematics 2017

Edited by Mircea Pitici

This annual anthology brings together the year's finest mathematics writing from around the world. Featuring promising new voices alongside some of the foremost names in the field, The Best Writing on Mathematics 2017 makes available to a wide audience many articles not easily found anywhere else.

Paper $\$ 24.95$

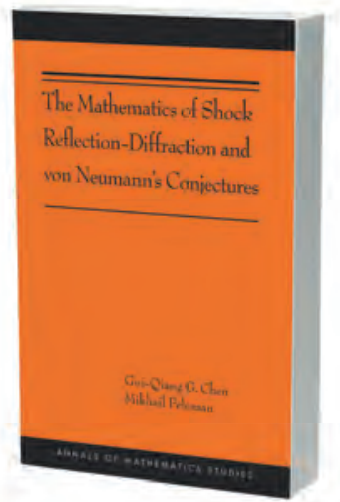

The Mathematics of Shock Reflection-Diffraction and von Neumann's Conjectures

Gui-Qiang G. Chen \& Mikhail Feldman

This book offers a survey of recent developments in the analysis of shock reflection-diffraction, a detailed presentation of original mathematical proofs of von Neumann's conjectures for potential flow, and a collection of related results and new techniques in the analysis of partial differential equations (PDEs), as well as a set of fundamental open problems for further devlopment. Paper $\$ 75.00$ 


\title{
Writing a Teaching Letter
}

Chris Woodward

\author{
Communicated by Harriet Pollatsek
}

Note: The opinions expressed here are not necessarily those of Notices.

Responses on the Notices webpage are invited.

Letters of recommendation that provide evidence of effective instruction are required by most departments as part of the hiring process. Many of us on our departmental hiring committee read some of the teaching letters we received this year with alarm. A number of these letters contained evidence of effective instruction that was anecdotal, circumstantial, or at worst didn't mention teaching at all. The purpose of this note is to offer some suggestions to our colleagues about what I think a helpful teaching letter should contain.

First, a teaching letter should describe what courses the candidate has taught, as well as the other educational activities the candidate has been involved in. These might include mentoring students, supervising undergraduate research projects, or organizing seminars. The letter should also describe the approximate sizes of the classes taught and the candidate's role. Is the candidate experienced at teaching large classes or small tutorials? Was the candidate a recitation instructor or the course head?

Second, the letter should contain exact quotes from students, or at least a description of student feedback. Usually in the American system exact quotes from students are available to copy and paste into the letter from the student

Chris Woodward is faculty in the Department of Mathematics at Rutgers University, New Brunswick. His e-mail address is woodwardc@gmai 1 . com.

For permission to reprint this article, please contact: reprint-permission@ams.org.

DOI: http://dx.doi.org/10.1090/noti1587 evaluations at the end of the semester. If not, the writer may need to ask for help from some former students of the candidate. If there are negative comments, the writer should try to give the committee an idea of why without being unnecessarily harsh.

Third, the letter should contain some numerical data about teaching effectiveness, a description of the scale (typically one to five, with five highest), and the average score in the department. ${ }^{1}$ If no numerical data are given, a hiring committee might reasonably assume that the evaluations were very low. If the candidate taught a large number of classes, the letter might give the range of scores and a description of the most recent few.

Fourth, the letter should contain comments from at least one and preferably at least two faculty members who visited the candidate's class, recitation, or tutorial; this could but need not be the letter writer. How was the board work? The pacing? The spoken English? The mathematical accuracy? The ability of the instructor to engage the entire class? If the letter writer hasn't visited the candidate's class, he or she should try to find a colleague who did. (If no one did, the department is doing a poor job of mentoring.)

${ }^{1}$ Note that evidence of correlation between student ratings and student learning is highly questionable; see [UWG]. However, for the moment, use of student effectiveness scores in hiring and promotion decisions is almost ubiquitous. If the writer objects to the use of such data, and as a result does not include it, this should perhaps be explained in the teaching letter. 
Fifth, the letter should mention at the top any teaching awards. There are certain jobs that are available only to those who have won such a distinction or at least have outstanding teaching credentials, and it helps hiring committees sort the applications if this is mentioned at the beginning.

A letter that addresses all of the above issues positively can produce a substantial upward bump for the candidate in the rankings. It also provides a valuable service to hiring departments.

Finally, the candidate might want to provide the letter writer with as much of the material mentioned above as possible. This might include a list of educational and mentorship activities as well as a list of faculty who have visited classes or tutorials. The letter might be requested in the summer, when the letter writer has time to gather the data and is not yet swamped with letter requests.

\section{Reference}

[UWG] UTTL, White, AND GonzALEz, Meta-Analysis of Faculty's

Teaching Effectiveness: Student Evaluation of Teaching Ratings and Student Learning Are Not Related, Stud. Educ. Eval. 54 (2017).

\section{Photo Credit}

Photo of Chris Woodward by Neepa Maitra.

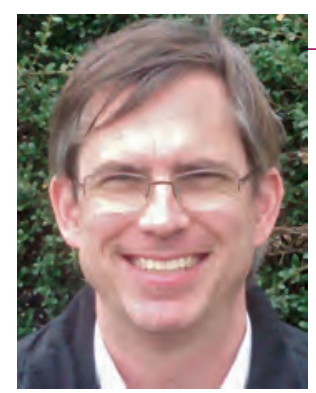

\section{ABOUT THE AUTHOR}

Chris Woodward has served on his department's hiring committee for at least ten years and has served for three years as the faculty member responsible for writing teaching letters for graduate students.

\section{Chris Woodward}

American Mathematical Society

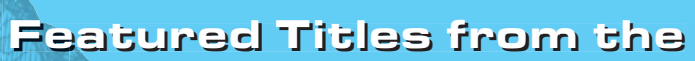

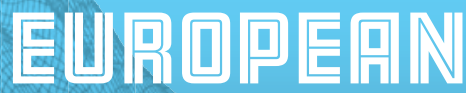

\section{MATHEMPITIERL} 50데퓬

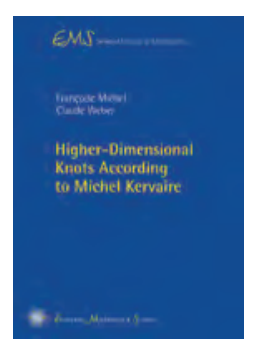

\section{Higher-Dimensional Knots According to Michel Kervaire}

Françoise Michel, Université Paul Sabatier, Toulouse, France, and Claude Weber, Université de Genève, Switzerland

Michel Kervaire wrote six papers which can be considered fundamental to the development of higher-dimensional knot theory. They are not only of historical interest but naturally introduce some of the essential techniques in this fascinating theory. This book provides graduate students with the basic concepts necessary to read texts in higher-dimensional knot theory and its relations with singularities.

EMS Series of Lectures in Mathematics, Volume 28; 2017; 144 pages; Softcover; ISBN: 978-3-03719-180-4; List US\$38; AMS members US $\$ 30.40$; Order code EMSSERLEC $/ 28$

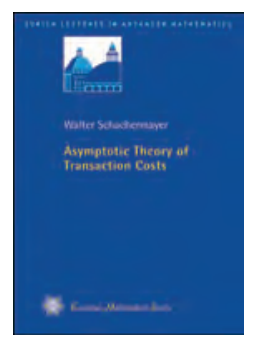

\section{Asymptotic Theory of Transaction Costs}

Walter Schachermayer, University of Vienna, Austria

A classical topic in mathematical finance is the theory of portfolio optimization. These lecture notes present some striking recent results of the asymptotic dependence of the relevant quantities when transaction costs tend to zero.

Zurich Lectures in Advanced Mathematics, Volume 23; 2017; 160 pages; Softcover; ISBN: 978-3-03719-173-6; List US\$38; AMS members US\$30.40; Order code EMSZLEC/23

Publications of the European Mathematical Society (EMS). Distributed within the Americas by the American Mathematical Society.

\section{Explore more titles at} bookstore.ams.org. (iii)AMS Bookstore bookstore.ams.org 


\title{
Accessible Mathematics for People with Hearing Loss at Colleges and Universities
}

\author{
J. Tilak Ratnanather
}

Communicated by Steven J. Miller

\section{Note: The opinions expressed here are not necessarily those of Notices. Responses on the Notices webpage are invited.}

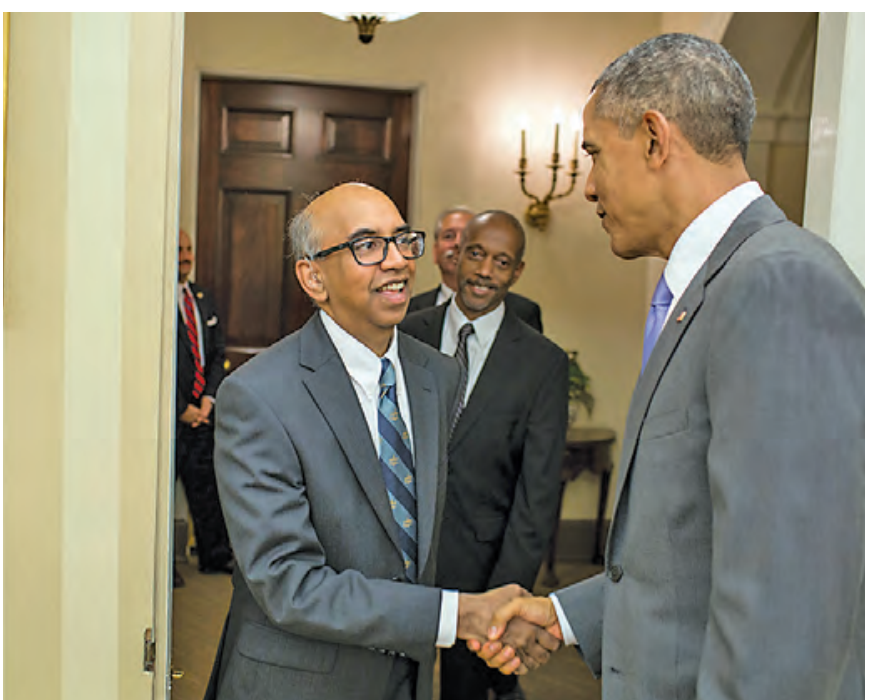

President Obama congratulates Ratnanather on the Presidential Award for Excellence in Science, Mathematics, and Engineering Mentoring

Tilak Ratnanather is associate research professor of biomedical engineering and is affiliated with the Center for Imaging Science and Institute for Computational Medicine at Johns Hopkins University. His e-mail address is tilak@cis.jhu.edu.

For permission to reprint this article, please contact: reprint-permission@ams.org.

DOI: http://dx.doi.org/10.1090/noti1588

\section{Introduction: Succeed in mathematics with hearing loss}

"Mathematics is the one subject in school that does not require hearing," wrote the late Dame Kathleen Ollerenshaw (Figure 1) [3]. She was the second person with hearing loss to obtain a doctoral degree in mathematics, at the University of Oxford in 1945. Among other things, she studied magic squares, including finding the first general solution for restoring the Rubik's ${ }^{\circledR}$ Cube. Ollerenshaw's striking statement portrays mathematics as a visual discipline that is naturally attractive to people with hearing loss. Yet few others with hearing loss have obtained doctoral degrees in mathematics. There were 13 in the United States from 2006 to 2010 [5], and at least 6 from the University of Oxford based on anecdotal information. It is remarkable that the first two were women. The

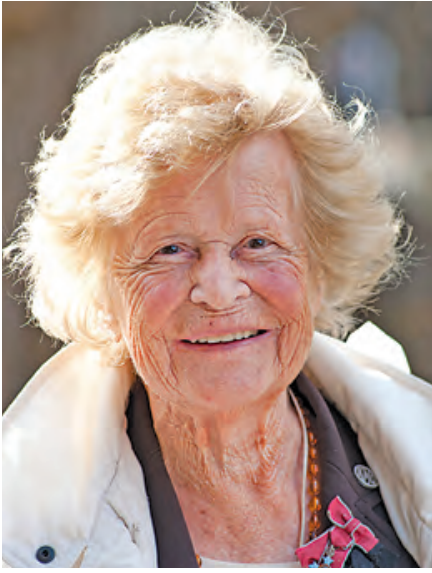

Figure 1. Dame Kathleen Ollerenshaw, the second person with hearing loss to obtain a doctoral degree in mathematics, wrote, "Mathematics is the one subject in school that does not require hearing." 


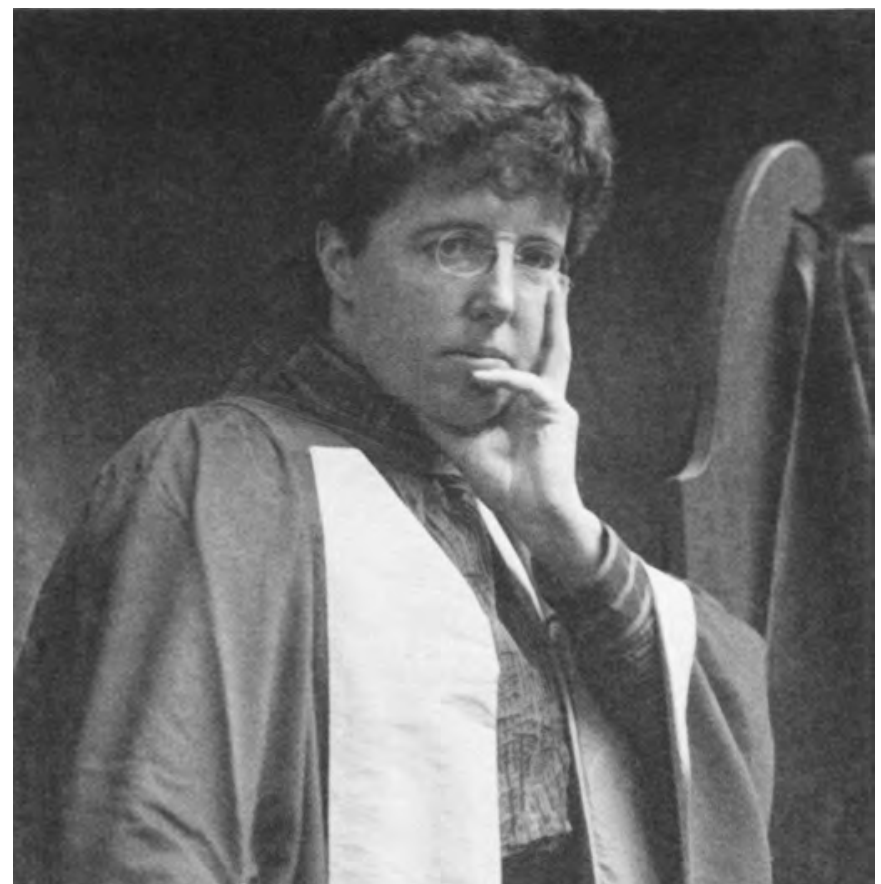

Figure 2. Charlotte Agnas Scott, the first person with hearing loss to obtain a doctoral degree in mathematics, headed the mathematics department at Bryn Mawr College, where she researched algebraic geometry and was a co-founder of the American Mathematical Society.

first was Charlotte Angas Scott (Figure 2) [2], who in 1880 was denied Eighth Wrangler ranking in the Cambridge Mathematical Tripos because she was female, but in 1885 obtained a DSc from the University of London, ironically for research at Cambridge. Scott became the first woman to head a mathematics department, at Bryn Mawr College, where she researched algebraic geometry and was a cofounder of the American Mathematical Society.

With the emergence of amplification technologies such as hearing aids and cochlear implants and accessibility legislation in some countries, more people with hearing loss are studying mathematics or using it in their teaching or research. While not all remain in mathematics, some go on to hold leadership positions in STEM (Science, Technology, Engineering, and Mathematics). One example is Vint Cerf (Figure 3), known as the Father of the Internet, who obtained an undergraduate degree in mathematics at Stanford University before pursuing his seminal doctoral research in computer science at UCLA. Another is Dimitri Kanevsky (Figure 4), who obtained a $\mathrm{PhD}$ in mathematics at Moscow State University before moving to the United States, where he carved out a productive career in speech recognition, first at IBM Research and now at Google. As these achievements indicate the potential for people with hearing loss to "focus on [their] strengths and talents"1 in acquiring mathematical knowledge, it is opportune to

$\sqrt[1]{\text { obamawhitehouse.archives.gov/realitycheck/ }}$ blog/2012/05/07/technology-change-great-equalizer.

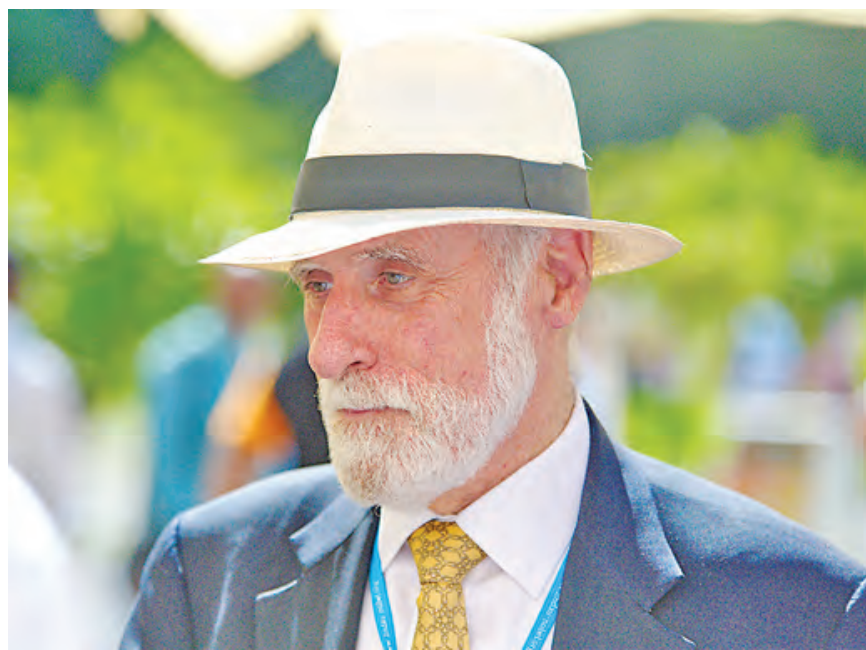

Figure 3. Vint Cerf, "Father of the Internet."

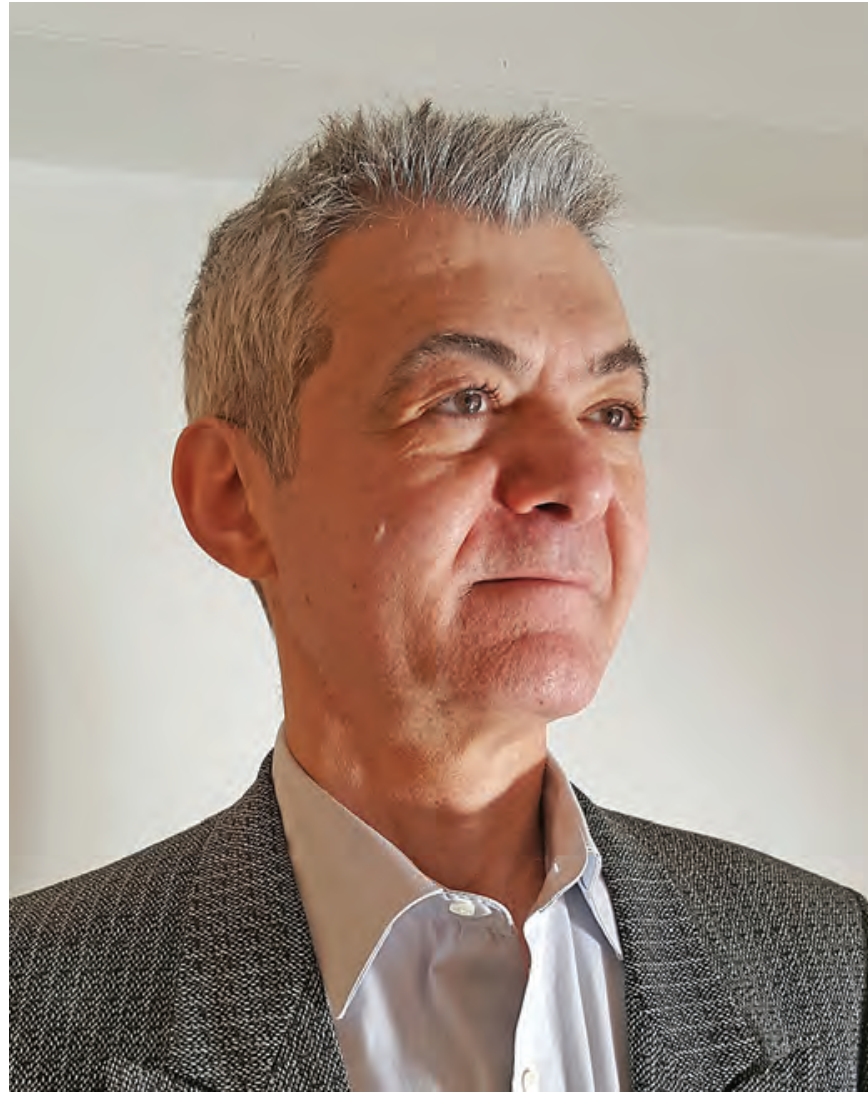

Figure 4. Dimitri Kanevsky advanced speech recognition, first at IBM Research and now at Google.

describe how mathematics can be made more accessible at colleges and universities.

\section{Hearing loss: Underrepresented in STEM}

Hearing loss cuts across society. According to the World Health Organization, hearing loss with auditory threshold elevations greater than $40 \mathrm{~dB}$ in the better ear has a global incidence of five percent. People with hearing loss struggle with reduced access to auditory information 
and have to deal with the societal challenges of isolation, ignorance, and invisibility. In the United States, the proportion of people in STEM with hearing loss is extremely small, only about one percent [5], partially due to low proportion among college students-those with hearing loss are estimated to number from 30,000 to 400,000, representing 0.3-4.0 percent of all college students. Hearing loss covers two broad spectra. First, the degree ranges from partial (which can be corrected with hearing aids) to profound (which in some cases can be mitigated with cochlear implants). Second, hearing loss can be congenital, progressive, or acquired. For example, Scott's hearing loss was acquired in childhood and became progressively profound while Ollerenshaw's was congenitally partial and then progressively profound. People with hearing loss hear sounds across a limited frequency range regardless of loudness, have trouble distinguishing speech in noise, and find it difficult to interpret speech from a sparse signal. As amplification elevates both speech and background noise, articulation becomes important. Therefore accessibility to mathematics for people with hearing loss at college and university must be in the context of their needs.

\section{Accessibility: Small steps, but leaps for mankind}

In the United States, 2,500-3,000 college students with hearing loss attend specialist tertiary institutions, where they receive a whole range of support services such as sign language interpreters, lipspeakers, and captioning [1]. But support services at mainstream colleges attended by the other 96-99 percent of college students with hearing loss vary in terms of depth and coverage [3]. Further, professors with hearing loss, who number in the hundreds worldwide, experience a different set of problems [4]. Both groups have common accessibility needs.

The acoustic environment is important. Ambient noises such as traffic, people walking and talking, moving furniture, rustling paper, and air conditioning can be difficult to filter out while focusing on speech in conversations, lectures, and seminars. Soundproofing such as carpeting and low ceilings helps to mitigate effects of reverberations, while offices with an open plan are disastrous. Clear speech should be encouraged: carefully enunciated words can go a long way to maximize learning and the distillation of essential points. So can concise, clear presentations. Good lighting, balanced between the speaker's face and the projector screen, also helps. Above all, interactive whiteboards, blackboards, and flip charts facilitate twoway communication. All of this applies not only in classroom lectures, but also in seminars and podium talks at conferences and workshops.

Technology can help. If amplification is used, clear speech can be enhanced by assistive listening devices, such as inductive loop, FM, WiFi, and infrared systems. I vividly recall the clarity of a university lecturer using an FM system; there were a few occasions when I anticipated what was going to be said. This is clearly a cognitive benefit of the six dB gain provided by these devices. Nowadays with group discussions and the proliferation of the flipped classroom, it is common to be seated around a table. Here a flip chart and a sound field system help, especially if pauses are taken before speaking with a microphone. Online lecture notes help, especially when accompanied by write-ups by note-takers who pick up the interesting digressions.

Real-time captioning is now available on-site or remotely. A transcriber receives the speech signal and converts it to text. Technical words can be communicated in advance to the captioner for assembling a dictionary that deepens over time. With rapid advances in hardware and software, captioning and speech-to-text tablet applications will help to level the playing field in the years to come. Interactive content produced by captioned MOOCs has an enormous potential to facilitate learning and teaching by everyone and provide more time for personal contact.

\section{Strategies: Thinking outside the box}

Hearing loss is personal, so what works for one may not work for another. Thinking outside the box can yield solutions to the benefit of all. Ian Shipsey, professor of experimental physics at the University of Oxford, goes to "extraordinary lengths to make [his] explanations clear so that the questions [from his students] would not be trivial ones."2 As a student, David James, now professor of mathematics at Howard University, found that "mistakes [were] productive; when [he] made one, the teacher could explain exactly why it was wrong and [he] could learn from that." ${ }^{3}$ In algebraic manipulations, it might be better to use phonetically distinct symbols. Only when I got my cochlear implant a few years ago was I able to differentiate the articulation of $\varphi$ and $v$ as the diffeomorphism and velocity associated with geodesics in shape spaces in computational anatomy. To deal with questions in a large room, Scott and Ollerenshaw were assisted by graduate students in classes [2] and hosts at seminars [3]; in my case, moderators at conferences have been helpful.

People with hearing loss often experience greater visual acuity. This may explain why people with hearing loss think differently, as in Ollerenshaw's use of subluminal thinking to solve problems [3] and Scott's preference for geometrical reasoning based on simple but rigorous proofs [2].

\section{Summary: Mind that person who may have hearing loss}

Making mathematics more accessible to people with hearing loss in colleges and universities should significantly improve the underrepresentation of people with hearing loss in STEM. In particular, enhanced ac-

4www.sciencemag.org/careers/2010/04/profiles-techno1ogica1-adaptation

${ }^{3}$ from The Voices of the Oral Deaf: Fourteen Role Models Speak Out by J. Reisler 
cessibility will go a long way to address the societal challenges of hearing loss. By far the two biggest challenges are invisibility and isolation, which cause hearing loss to be underreported. Though I was motivated, I knew only a few people with hearing loss who had succeeded in mathematics, so I had some doubts. Such doubts can be dispelled by knowing about and networking with mathematicians with hearing loss. The third challenge is ignorance due to lack of information about dealing with hearing loss, so it is important to self-advocate for accessibility. Having more people with hearing loss in mathematics, aided by advances in amplification and pedagogy, has the potential for new ideas and concepts in mathematics to emerge to the benefit of everyone.

ACKNOWLEDGMENT. I thank my mentors at University College London and University of Oxford, and fellow mathematicians too numerous to mention here for their support throughout my career.

\section{References}

[1] H. ADLER, B. JACOB, K. KuRZ, and R. KuSHALnAGAR, Undergraduate research in mathematics with deaf and hard-of-hearing students: four perspectives, Involve, a Journal of Mathematics 7 (2014), 247-264.

[2] P. C. Kenschaft, Charlotte Angas Scott, Women of Mathematics: A Biobibliographic Sourcebook, L. Grinstein and P. Campbell, ed., Greenwood Press, 1987.

[3] K. Ollerenshaw, To Talk of Many Things: An Autobiography, Manchester University Press, 2004.

[4] D. H. Smith and J. F. ANDREwS, Deaf and hard of hearing faculty in higher education: enhancing access, equity, policy, and practice, Disabil. Soc. 30 (2015), 1521-1536.

[5] C. M. Solomon, D. Braun, R. Kushalnagar, R. E. LADneR, D. LUNDBERG, R. PAINTER, and R. NUZzO, Workshop for emerging deaf and hard of hearing scientists (2012), www. washington. edu/accesscomputing/WhitePaper-Final_Ca11audet_ emerging_sci_2_15_13.pdf/.

Photo Credits

Photo of Ratnanather and President Obama courtesy of Pete Souza.

Figure 1 courtesy of Somerville College Archives, Oxford.

Figure 2 courtesy of Special Collections Department, Bryn Mawr College Library.

Figure 3 courtesy of Peter Badge.

Figure 4 courtesy of Dimitri Kanevsky.

Photo of Tilak Ratnanather courtesy of Will Kirk, Johns Hopkins University.

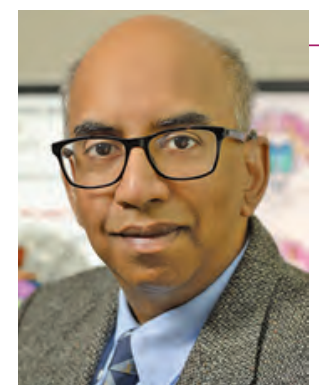

\section{ABOUT THE AUTHOR}

Born with profound bilateral hearing loss, Tilak Ratnanather has recruited or mentored more than 50 individuals with hearing loss worldwide in science, technology, engineering, and mathematics.
American Mathematical Society
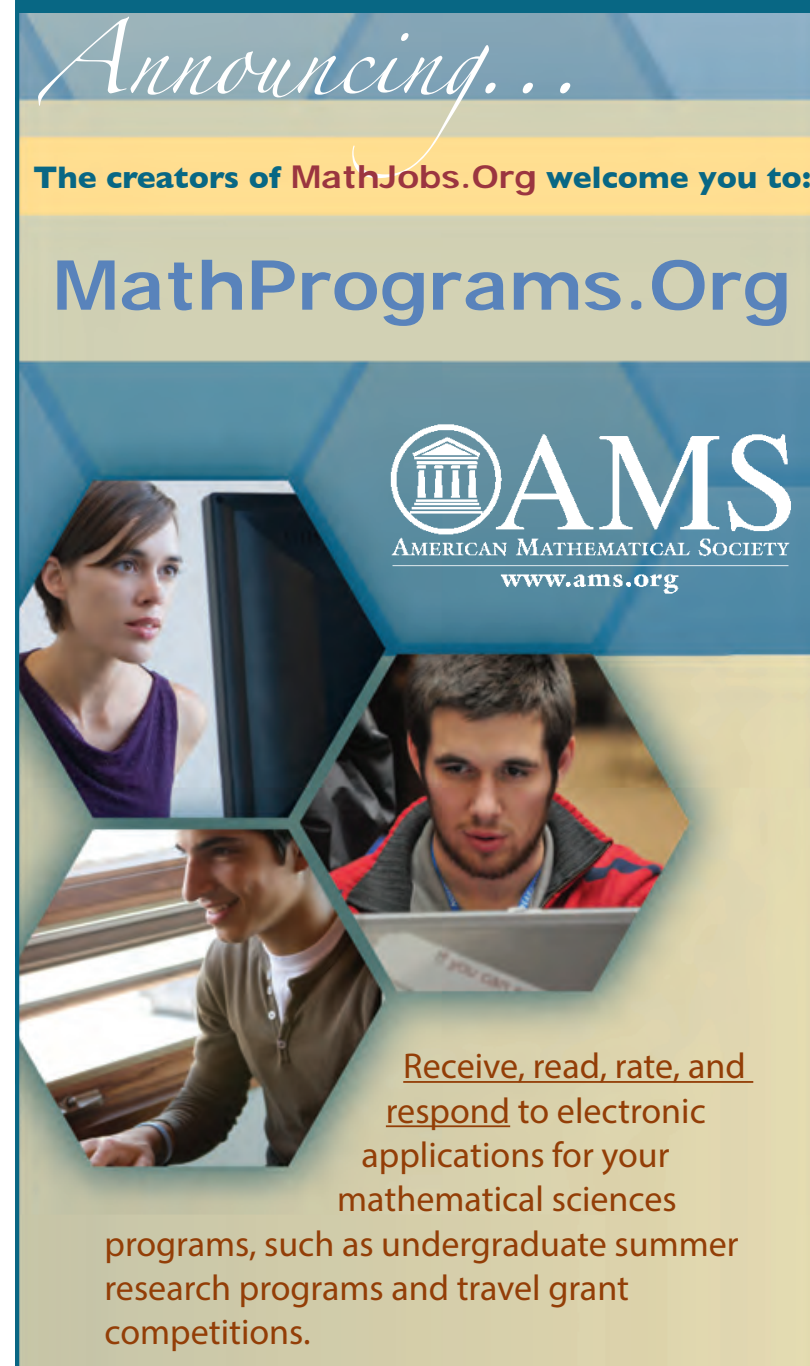

Customize your settings and control the application form; also set secure access for the admissions committee.

Enter program announcements for public display.

Download data to personal computers for use in word processing and spreadsheets or as a full permanent storage file.

\section{Service is FREE to applicants.}

Institutions pay annually for one program or for multiple programs. 


\section{FROM THE AMS SECRETARY}

\section{Backlog of Mathematics Research Journals}

\begin{tabular}{|c|c|c|c|c|c|c|c|}
\hline \multirow{3}{*}{$\begin{array}{l}\text { Journal (Print } \\
\text { and Electronic) }\end{array}$} & \multirow{3}{*}{$\begin{array}{l}\text { Number } \\
\text { issues } \\
\text { per Year }\end{array}$} & \multirow{3}{*}{$\begin{array}{l}\text { Approximate } \\
\text { Number } \\
\text { Pages } \\
\text { per Year } \\
\end{array}$} & \multicolumn{3}{|c|}{$\begin{array}{l}2016 \text { Median Time } \\
\text { (in Months) from: }\end{array}$} & \multirow{2}{*}{\multicolumn{2}{|c|}{$\begin{array}{l}\text { Current Estimate of } \\
\text { Waiting Time between } \\
\text { Submission and Publication } \\
\text { (in Months) }\end{array}$}} \\
\hline & & & \multirow{2}{*}{$\begin{array}{c}\text { Submission } \\
\text { to Final } \\
\text { Acceptance }\end{array}$} & \multirow{2}{*}{\begin{tabular}{|c} 
Acceptance \\
to \\
Print
\end{tabular}} & \multirow{2}{*}{$\mid \begin{array}{l}\text { Acceptance } \\
\text { to Electronic } \\
\text { Posting }\end{array}$} & & \\
\hline & & & & & & Print & Electronic \\
\hline Acta Inform. & 8 & 750 & 11 & 11 & 1 & 23 & 12 \\
\hline Adv. in Appl. Math. & 10 & 1615 & 7 & 3 & 0.7 & 11 & 8 \\
\hline Adv. Math. & 18 & 17752 & 15 & 3 & 1.5 & 17 & 15 \\
\hline Algebr. Geom. Topol. & 6 & 4000 & 9 & 10 & 9 & 19 & 18 \\
\hline Algebra Number Theory & 10 & 2500 & 9 & 4 & 3 & 13 & 12 \\
\hline Algorithmica & 12 & 3000 & 9 & 10 & 1 & 18 & 10 \\
\hline Amer. J. Math. & 6 & 1728 & NR & NA & NA & 18 & $16-17$ \\
\hline Anal. PDE & 8 & 2000 & 8 & 5 & 4 & 12 & 11 \\
\hline Ann. Appl. Probab. & 6 & 3900 & 10 & 11.5 & 11.5 & 22.5 & 22.5 \\
\hline $\begin{array}{l}\text { Ann. Inst. H. Poincare Anal. Non } \\
\text { Lineaire }\end{array}$ & 6 & 1662 & 9 & 17 & 2.2 & 22 & 10 \\
\hline Ann. K-Theory & 4 & 800 & 8 & 5 & 3 & 9 & 13 \\
\hline Ann. Mat. Pura Appl. (4) & 6 & 2000 & 7 & 14 & 1 & 21 & 8 \\
\hline Ann. of Math. (2) & 6 & 2100 & 11 & 6 & 4 & 11 & 5 \\
\hline Ann. Polon. Math & 8 & 800 & 6.6 & 4.4 & 3.3 & 8 & 6 \\
\hline Ann. Probab. & 6 & 4200 & 12.5 & 14.5 & 14.5 & 29 & 29 \\
\hline Ann. Pure Appl. Logic & 12 & 1640 & 13 & 6 & 4 & 18 & 16 \\
\hline Ann. Statist. & 6 & 3600 & 6.5 & 6.5 & 6.5 & 23.5 & 23.5 \\
\hline Appl. Anal. & 12 & 2944 & 4 & 11 & 1 & 15.7 & 5.5 \\
\hline Appl. Comput. Harmon. Anal. & 6 & 1453 & 9 & 11 & 1.1 & 26 & 9 \\
\hline Appl. Math. Comput. & 24 & 8102 & 7 & 2 & 1 & 11 & 10 \\
\hline Arch. Hist. Exact. Sci. & 6 & 650 & 3 & 8 & 1 & 12 & 4 \\
\hline Arch. Math. Logic & 8 & 950 & 16 & 4 & 1 & 20 & 17 \\
\hline Arch. Ration. Mech. Anal. & 12 & 5500 & 12 & 5 & 1 & 17 & 13 \\
\hline Automatica J. IFAC & 12 & 3881 & 13 & 4 & 2.1 & 17 & 17 \\
\hline Balkan J. Geom. Appl. & 2 & 240 & 5 & 5 & 3 & 8 & 6 \\
\hline
\end{tabular}

The Backlog of Research Journals is reported each year in the November issue of the Notices. The report covers journals of publishers who have agreed to participate and who continue to provide backlog information. Publishers whose journals are not currently included can request that their journals be added. Such requests should be made in e-mail to Marcia Almeida, back1ogreport@ams.org. To be eligible for inclusion in the backlog report, a journal must be on the list of journals receiving cover-to-cover treat- ment in Mathematical Reviews (www.ams.org/msnhtm1/ serials.pdf).

Once a publisher's journals are accepted for inclusion, the publisher must designate a contact person or persons to supply data about the journals to the AMS. While the AMS makes every effort to obtain the data from the designated contacts, if data about a journal is not supplied, then that journal will not appear in the backlog report. 
FROM THE AMS SECRETARY

\begin{tabular}{|c|c|c|c|c|c|c|c|}
\hline \multirow{3}{*}{$\begin{array}{l}\text { Journal (Print } \\
\text { and Electronic) }\end{array}$} & \multirow{3}{*}{$\begin{array}{c}\text { Number } \\
\text { issues } \\
\text { per Year }\end{array}$} & \multirow{3}{*}{$\begin{array}{l}\text { Approximate } \\
\text { Number } \\
\text { Pages } \\
\text { per Year } \\
\end{array}$} & \multicolumn{3}{|c|}{$\begin{array}{l}2016 \text { Median Time } \\
\text { (in Months) from: }\end{array}$} & \multirow{2}{*}{\multicolumn{2}{|c|}{$\begin{array}{c}\text { Current Estimate of } \\
\text { Waiting Time between } \\
\text { Submission and Publication } \\
\text { (in Months) }\end{array}$}} \\
\hline & & & $\begin{array}{c}\text { Submission } \\
\text { to Final }\end{array}$ & $\mid \begin{array}{c}\text { Acceptance } \\
\text { to }\end{array}$ & $\begin{array}{l}\text { Acceptance } \\
\text { to Electronic }\end{array}$ & & \\
\hline & & & & & & Print & Electronic \\
\hline Beitr. Algebra Geom. & 4 & 850 & 6 & 10 & 1 & 17 & 7 \\
\hline Bernoulli & 4 & 4000 & 10.5 & 10.5 & 10.5 & 26 & 26 \\
\hline Bull. Aust. Math. Soc. & 6 & 1056 & 0.5 & 8 & 3 & 9 & 4 \\
\hline Bull. Lond. Math. Soc. & 6 & 1152 & 7.9 & 3.2 & 1.7 & 11.6 & 10.3 \\
\hline Bull. Sci. Math. & 8 & 997 & 7 & 10 & 2.6 & 15 & 14 \\
\hline $\begin{array}{l}\text { Calc. Var. Partial Differential } \\
\text { Equations }\end{array}$ & 6 & 3900 & 9 & 3 & 1 & 12 & 10 \\
\hline Canad. J. Math. & 6 & 1440 & 7 & 11 & 5 & 18 & 13 \\
\hline Canad. Math. Bull. & 4 & 896 & 5 & 8 & 2 & 14 & 7 \\
\hline Combinatorica & 6 & 750 & 8 & 28 & 15 & 25 & 10 \\
\hline Comm. Math. Phys. & 24 & 8200 & 13 & 5 & 1 & 17 & 14 \\
\hline Commun. Appl. Math. Comput. Sci. & 2 & 250 & 10 & 4 & 3 & 17 & 16 \\
\hline Commun. Pure Appl. Anal. & 6 & 2500 & 4 & 3 & 1 & 9 & 7 \\
\hline Complex Var. Elliptic Equ. & 12 & 1800 & 4.4 & 5.4 & 1.5 & 11.7 & 6.6 \\
\hline Compos. Math. & 12 & 2688 & 10 & 7.7 & 5.7 & 18.6 & 15.5 \\
\hline Comput. Aided Geom. Design & 9 & 1064 & 5 & 3 & 0.9 & 4 & 4 \\
\hline Comput. Geom. & 9 & 497 & 14 & 3 & 0.5 & 14 & 13 \\
\hline Comput. Math. Appl. & 24 & 5561 & 7 & 2 & 1.1 & 9 & 8 \\
\hline Comput. Methods Funct. Theory & 4 & 650 & 6 & 10 & 1 & 16 & 7 \\
\hline Computing & 12 & 1250 & 8 & 14 & 1 & 22 & 9 \\
\hline Constr. Approx. & 6 & 1000 & 10 & 11 & 2 & 16 & 9 \\
\hline Des. Codes Cryptogr. & 12 & 2700 & 6 & 9 & 1 & 18 & 8 \\
\hline Differential Geom. Appl. & 6 & 1464 & 9 & 3 & 0.8 & 11 & 10 \\
\hline Discrete Appl. Math & 18 & 4852 & 12 & 7 & 1.2 & 15 & 14 \\
\hline Discrete Contin. Dyn. Syst. & 12 & 7000 & 5 & 3 & 6 & 8 & 6 \\
\hline Discrete Contin. Dyn. Syst. Ser. B & 10 & 4000 & 5 & 3 & 6 & 8 & 6 \\
\hline Discrete Math. & 12 & 2452 & 11 & 3 & 1.4 & 14 & 12 \\
\hline Discrete Optim. & 4 & 780 & 13 & 6 & 1.2 & 12 & 11 \\
\hline Duke Math.J. & 18 & 3600 & 15 & 10 & 7 & 23 & 20 \\
\hline Dyn. Syst. & 4 & 592 & 6.3 & 9.2 & 1.2 & 14 & 7.7 \\
\hline European J. Combin. & 8 & 1373 & 10 & 2 & 1.2 & 13 & 11 \\
\hline Expo. Math. & 4 & 471 & 7 & 10 & 0.9 & 16 & 8 \\
\hline Finite Fields Appl. & 6 & 1354 & 8 & 2 & 0.8 & 11 & 9 \\
\hline Found. Comput. Math. & 6 & 1300 & 6 & 12 & 1 & 18 & 7 \\
\hline Geom. Dedicata & 6 & 1753 & 283.9 & $6-8$ & 0.5 & 8 & $1-2$ \\
\hline Geom. Topol. & 6 & 4000 & 10 & 12 & 11 & 21 & 20 \\
\hline Graphs Combin. & 6 & 2467 & 8.6 & 1.5 & 0.6 & 6 & 5 \\
\hline Historia Math. & 4 & 465 & 9 & 6 & 1.1 & 11 & 8 \\
\hline Homology Homotopy Appl. & 2 & 800 & 8.3 & 10.3 & 7.3 & 17 & 14 \\
\hline Houston J. Math. & 4 & 1400 & 8 & 21 & 18 & 21 & 18 \\
\hline Illinois J. Math. & 4 & 1200 & 6 & 4 & 4 & 10 & 9 \\
\hline Indag. Math. (N.S.) & 6 & 1335 & 6 & 5 & 1.1 & 12 & 9 \\
\hline Indiana Univ. Math. J. & 6 & 2100 & 21 & 14 & $*$ & 23 & 4 \\
\hline Infor. Process. Lett. & 12 & 715 & 10 & 2 & 0.7 & 15 & 14 \\
\hline Invent. Math. & 12 & 3800 & 14 & 10 & 1 & 24 & 15 \\
\hline Involve & 5 & 900 & 5 & 10 & 9 & 15 & 14 \\
\hline Israel J. Math. & 6 & 4500 & 6 & 14 & 14 & 18 & 18 \\
\hline J. Algebra & 24 & 11626 & 10 & 3 & 1.6 & 13 & 11 \\
\hline
\end{tabular}


FROM THE AMS SECRETARY

\begin{tabular}{|c|c|c|c|c|c|c|c|}
\hline \multirow{3}{*}{$\begin{array}{l}\text { Journal (Print } \\
\text { and Electronic) }\end{array}$} & \multirow{3}{*}{$\begin{array}{l}\text { Number } \\
\text { issues } \\
\text { perYear }\end{array}$} & \multirow{3}{*}{$\begin{array}{l}\text { Approximate } \\
\text { Number } \\
\text { Pages } \\
\text { perYear } \\
\end{array}$} & \multicolumn{3}{|c|}{$\begin{array}{l}2016 \text { Median Time } \\
\text { (in Months) from: }\end{array}$} & \multirow{2}{*}{\multicolumn{2}{|c|}{$\begin{array}{l}\text { Current Estimate of } \\
\text { Waiting Time between } \\
\text { Submission and Publication } \\
\text { (in Months) }\end{array}$}} \\
\hline & & & \multirow{2}{*}{$\begin{array}{c}\text { Submission } \\
\text { to Final } \\
\text { Acceptance }\end{array}$} & \multirow{2}{*}{\begin{tabular}{|} 
Acceptance \\
to \\
Print
\end{tabular}} & \multirow{2}{*}{\begin{tabular}{|c} 
Acceptance \\
to Electronic \\
Posting
\end{tabular}} & & \\
\hline & & & & & & Print & Electronic \\
\hline J. Algebraic Geom. & 4 & 800 & 12 & 16 & 2 & 16 & 12 \\
\hline J. Amer. Math. Soc. & 4 & 1200 & 19 & 12.3 & 1.6 & 28.1 & 19 \\
\hline J. Anal. Math. & 3 & 1600 & 5 & 30 & 30 & NR & NR \\
\hline J. Appl. Log. & 6 & 782 & 7 & 5 & 1.7 & 15 & 14 \\
\hline J. Approx. Theory & 12 & 1441 & 10 & 3 & 1.6 & 12 & 11 \\
\hline J. Aust. Math. Soc. & 6 & 864 & 6 & 10 & 5 & 21 & 9.5 \\
\hline J. Combin. Theory Ser. A & 8 & 2050 & 14 & 2 & 0.8 & 16 & 14 \\
\hline J. Combin. Theory Ser. B & 6 & 2101 & 23 & 4 & 0.9 & 25 & 23 \\
\hline J. Complexity & 6 & 861 & 6 & 4 & 1.1 & 10 & 7 \\
\hline J. Comput. Appl. Math. & 18 & 6133 & 9 & 3 & 1.3 & 12 & 10 \\
\hline J. Comput. System Sci. & 8 & 1506 & 17 & 4 & 2.7 & 19 & 18 \\
\hline J. Convex Anal. & 4 & 1400 & 8 & 10 & 1 & 12 & 7 \\
\hline J. Difference Equ. Appl. & 12 & 2112 & 5.4 & 6.9 & 1 & 12.4 & 6.7 \\
\hline J. Differential Equations & 24 & 15949 & 6 & 2 & 0.6 & 9 & 8 \\
\hline J. Differential Geom. & 9 & 1625 & 24 & 10 & 20 & 14 & 16 \\
\hline J. Eur. Math. Soc. (JEMS) & 12 & 3000 & 10 & 20 & 19 & 24 & 23 \\
\hline J. Funct. Anal. & 24 & 8420 & 11 & 2 & 0.7 & 13 & 11 \\
\hline J. Geom. Phys. & 12 & 2444 & 8 & 3 & 1 & 12 & 10 \\
\hline J. Ind. Manag. Optim. & 4 & 1600 & 6 & 3 & 7 & 9 & 7 \\
\hline J. Integral Equations Appl. & 4 & 600 & 10 & 13 & 11 & 12 & 10 \\
\hline J. Lie Theory & 4 & 1200 & 6 & 9 & 1 & 14 & 6 \\
\hline J. Log. Algebr. Program. & 8 & 1472 & 12 & 7 & 4 & 15 & 12 \\
\hline J. Lond. Math. Soc. (2) & 6 & 2064 & 9.2 & 4.3 & 2.2 & 12 & 11 \\
\hline J. Math. Anal. Appl. & 24 & 15173 & 7 & 2 & 0.7 & 8 & 7 \\
\hline J. Math. Biol. & 14 & 3600 & 10 & 9 & 1 & 19 & 11 \\
\hline J. Math. Ecom. & 8 & 839 & 9 & 3 & 2 & 15 & 13 \\
\hline J. Math. Phys. & 12 & $8000-9000$ & 5.7 & 1 & 0.8 & 6.5 & 6 \\
\hline J. Math. Pures Appl. (9) & 12 & 1923 & 8 & 5 & 2.2 & 16 & 11 \\
\hline J. Math. Soc. Japan & 4 & 1800 & 8 & 17 & 17 & 25 & 25 \\
\hline J. Mod. Dyn. & $* *$ & 600 & 6 & 6 & 1 & 12 & 7 \\
\hline J. Multivariate Anal. & 10 & 2512 & 11 & 4 & 1.2 & 13 & 11 \\
\hline J. Number Theory & 12 & 5957 & 6 & 4 & 2.2 & 9 & 7 \\
\hline J. Operator Theory & 4 & 1000 & 6 & 8 & 7 & 12 & 11 \\
\hline J. Pseudo-Differ. Oper. Appl. & 4 & 550 & 3 & 9 & 1 & 8 & 5 \\
\hline J. Pure Appl. Algebra & 12 & 3338 & 9 & 3 & 0.7 & 14 & 11 \\
\hline J. Statist. Plann. Inference & 12 & 1322 & 8 & 3 & 1.3 & 10 & 9 \\
\hline J. Symbolic Logic & 4 & 1600 & 10 & 10 & 10 & 18 & 16 \\
\hline J. Théor. Nombres Bordeaux & 3 & 800 & 14 & 14 & 12 & 24 & 15 \\
\hline J. Theoret. Probab. & 4 & 1736 & 10 & NR & 10 & NR & NR \\
\hline J. Topol. & 4 & 1120 & 8.8 & 5.4 & 2.6 & 15.6 & 14.5 \\
\hline Kodai Math. J. & 3 & 600 & 5 & 7 & 7 & 12 & 12 \\
\hline Kyoto J. Math. & 4 & 900 & 4 & 13 & 13 & 21 & 17 \\
\hline Linear Algebra Appl. & 24 & 9399 & 7 & 3 & 0.7 & 9 & 8 \\
\hline Linear Multilinear Algebra & 12 & 2592 & 4.1 & 8.8 & 1 & 13.7 & 5.5 \\
\hline Lobachevskii J. Math. & 6 & 1000 & 4 & 5 & 5 & 5.2 & 5.2 \\
\hline Manuscripta Math. & 12 & 1600 & 9 & 7 & 1 & 16 & 10 \\
\hline Math. Ann. & 12 & 4800 & 11 & 11 & 1 & 22 & 12 \\
\hline
\end{tabular}


FROM THE AMS SECRETARY

\begin{tabular}{|c|c|c|c|c|c|c|c|}
\hline \multirow{3}{*}{$\begin{array}{l}\text { Journal (Print } \\
\text { and Electronic) }\end{array}$} & \multirow{3}{*}{$\begin{array}{l}\text { Number } \\
\text { issues } \\
\text { per Year }\end{array}$} & \multirow{3}{*}{$\begin{array}{l}\text { Approximate } \\
\text { Number } \\
\text { Pages } \\
\text { perYear } \\
\end{array}$} & \multicolumn{3}{|c|}{$\begin{array}{l}2016 \text { Median Time } \\
\text { (in Months) from: }\end{array}$} & \multirow{2}{*}{\multicolumn{2}{|c|}{$\begin{array}{c}\text { Current Estimate of } \\
\text { Waiting Time between } \\
\text { Submission and Publication } \\
\text { (in Months) }\end{array}$}} \\
\hline & & & $\begin{array}{l}\text { Submission } \\
\text { to Final }\end{array}$ & $\begin{array}{c}\text { Acceptance } \\
\text { to }\end{array}$ & $\begin{array}{l}\text { Acceptance } \\
\text { to Electronic }\end{array}$ & & \\
\hline & & & Acceptance & & Posting & Print & Electronic \\
\hline Math. Comp. & 6 & 3000 & 11.1 & 16.5 & 8.7 & 26.8 & 18.5 \\
\hline Math. Control Signals Systems & 4 & 700 & 12 & 2 & 0.7 & 12 & 10.5 \\
\hline Math. Oper. Res. & 4 & 1200 & 16.3 & 8.84 & 3 & 15 & 9 \\
\hline Math. Program. & 12 & 3400 & 17 & 12 & 1 & 29 & 18 \\
\hline Math. Res. Lett. & 6 & 1300 & 7 & 9 & 9 & 9 & 9 \\
\hline Math. Scand. & 4 & 640 & 7 & 27 & 25 & 29 & 27 \\
\hline Math. Social Sci. & 6 & 680 & 11 & 3 & 1.2 & 16 & 15 \\
\hline Math. Z. & 12 & 3500 & 13 & 6 & 1 & 19 & 14 \\
\hline Mathematika & 3 & 960 & 6.3 & 7.3 & 4.4 & 10.4 & 6.8 \\
\hline Mem. Amer. Math. Soc. & 6 & 4600 & 11.7 & 23 & NA & 38 & NA \\
\hline Methods Appl. Anal. & 4 & 400 & 5 & 4 & 4 & 7 & 7 \\
\hline Michigan Math.J. & 4 & 896 & 10 & 7 & 6 & 10 & 6 \\
\hline Monatsh. Math. & 12 & 2400 & 8 & 10 & 1 & 18 & 9 \\
\hline Multiscale Model. Simul. & 4 & 1550 & 9 & 4.6 & 2.6 & 13.6 & 11.6 \\
\hline Nagoya Math. J. & 4 & 820 & 8 & 4 & 2 & 7 & 6 \\
\hline Nonlinear Anal. & 18 & 4979 & 4 & 3 & 1.3 & 8 & 5 \\
\hline Nonlinear Anal. Hybrid Syst. & 4 & 884 & 10 & 5 & 1.3 & 13 & 10 \\
\hline Nonlinear Anal. Real World Appl. & 6 & 2393 & 7 & 3 & 1.1 & 10 & 9 \\
\hline Notre Dame J. Form. Log. & 4 & 600 & 11 & 29 & 26 & 31 & 28 \\
\hline Numer. Math. & 12 & 2500 & 14 & 11 & 1 & 26 & 15 \\
\hline Pacific J. Math. & 12 & 3000 & 6 & 7 & 6 & 13 & 12 \\
\hline Probab. Theory Related Fields & 12 & 3200 & 14 & 14 & 1 & 18 & 15 \\
\hline Proc. Amer. Math. Soc. & 12 & 5240 & 5.3 & 9.6 & 3.7 & 13.2 & 8.2 \\
\hline Proc. Lond. Math. Soc. (3) & 12 & 2496 & 10.6 & 3.1 & 1.8 & 13.8 & 10.8 \\
\hline Publ. Math. de l'IHES & 2 & 700 & 16 & 8 & 1 & 24 & 17 \\
\hline Quantum Topol. & 4 & 800 & 10 & 9 & 8 & 18 & 17 \\
\hline Quart. Appl. Math. & 4 & 800 & 1.7 & 13.3 & 11.5 & 8.2 & 2.6 \\
\hline Real Anal. Exchange & 2 & 500 & 7.3 & 11.7 & 8.2 & 20.8 & 16.7 \\
\hline Rocky Mountain J. Math. & 8 & 2800 & 9 & 28 & 26 & 22 & 20 \\
\hline Semigroup Forum & 6 & 1300 & 7 & 12 & 1 & 18 & 10 \\
\hline SIAM J. Appl. Math. & 6 & 2425 & 7 & 4.6 & 2.6 & 11.6 & 9.6 \\
\hline SIAM J. Comput. & 6 & 2225 & 19.5 & 4.8 & 2.8 & 24.3 & 22.3 \\
\hline SIAM J. Control Optim. & 6 & 3400 & 11.9 & 4.5 & 2.5 & 16.4 & 14.4 \\
\hline SIAM J. Discrete Math. & 4 & 2300 & 11 & 4.5 & 2.5 & 15.5 & 13.5 \\
\hline SIAM J. Math. Anal. & 6 & 4350 & 7.9 & 3.5 & 2.5 & 11.4 & 10.4 \\
\hline SIAM J. Matrix Anal. Appl. & 4 & 1775 & 8.9 & 4.5 & 2.5 & 13.4 & 11.4 \\
\hline SIAM J. Numer. Anal. & 6 & 3675 & 10.3 & 3.5 & 2.5 & 13.8 & 12.8 \\
\hline SIAM J. Optim. & 4 & 2900 & 11.9 & 4.6 & 2.6 & 16.5 & 14.5 \\
\hline SIAM J. Sci. Comput. & 6 & 6500 & 9.2 & 3.6 & 2.6 & 12.8 & 11.8 \\
\hline SIAM Rev. & 4 & 800 & 7.9 & 10.3 & 9.3 & 18.2 & 17.2 \\
\hline Stochastic Process. Appl. & 12 & 4005 & 12 & 6 & 2.2 & 17 & 11 \\
\hline Theory Comput. Syst. & 8 & 1500 & 8 & 8 & 1 & 18 & 9 \\
\hline Topology Appl. & 18 & 4267 & 7 & 4 & 2.6 & 11 & 11 \\
\hline Trans. Amer. Math. Soc. & 12 & 8880 & 7.9 & 23.4 & 14.5 & 30.6 & 21.3 \\
\hline
\end{tabular}




\section{FROM THE AMS SECRETARY}

\section{Journal (Electronic)}

Abstr. Appl. Anal. www. hindawi .com/journa1s/aaa/

Acta Math. Acad. Paedagog. Nyházi. (N.S.) www.emis.de/journals/AMAPN/

Adv. Difference Equ.

Tink.springer.com/journal/13662

Adv. Math. Commun. aimsciences.org/journa1s/home.jsp?journa1ID=10

Appl. Math. E-Notes

www. math. nthu. edu. tw/ amen/

Bound. Value Probl.

1ink. springer.com/journa1/13661

C. R. Math. Acad. Sci. Paris www. journals.elsevier.com/comptes-rendusmathematique/

Conform. Geom. Dyn.

www.ams.org/publications/journals/ journalsframework/ecgd

Differ. Geom. Dyn. Syst. www. mathem. pub.ro/dgds

Differ. Uravn. Protsessy Upr. www. math. spbu.ru/diffjournal/EN/about.htm7

Discrete Math. Theor. Comput. Sci. dmtcs . episciences.org/

Electron. J. Combin. www. combinatorics.org/

Electron. J. Differential Equations ejde.math.txstate.edu

Electron. J. Qual. Theory Differ. Equ. www. math.u-szeged.hu/ejqtde/

Electron. Res. Announc. Math. Sci. aimsciences. org/journa1s/home. jsp?journa1ID=14

Electron. Trans. Numer. Anal. etna.ricam. oeaw.ac.at/submissions/

ESAIM Control Optim. Calc. Var. Www.esaim-cocv.org/

ESAIM Math. Model. Numer. Anal. www.esaim-m2an.org/

ESAIM Probab. Stat. www. esaim-ps.org/

Fixed Point Theory Appl.

link.springer.com/journal/13663

Int. J. Math. Math. Sci. www. hindawi . com/journals/ijmms/

Int. J. Stoch. Anal. www. hindawi.com/journals/ijsa/

Integers

www. integers-ejcnt.org

Inverse Probl. Imaging aimsciences . org/journa1s/home.jsp?journa1ID=11

J. Appl. Math www. hindawi .com/journa1s/jam/

J. Inequal. Appl.

1ink.springer. com/journal/13660

J. Integer Seq. cs. uwaterloo.ca/journals/JIS/

\section{Number \\ of Articles \\ Posted in \\ 2016}

\section{3}

26

224

65

30

325

219

13

13

24

52

217

339

122
2016 Median Time (in days) from:

\begin{tabular}{c|c}
$\begin{array}{c}\text { Submission } \\
\text { to Final }\end{array}$ & Acceptance \\
to \\
Acceptance & Posting
\end{tabular}

Format(s)

html, pdf, ps, dvi, tex, ePUB

pdf, ps

html, pdf, tex

pdf

pdf

html, pdf

html, pdf

1.3

33

pdf

100

180

pdf

21

286

122

122

60

220

232

417

210

264.6

$344.5 \quad 127.8$

146

15

\section{html, pdf, tex, doc}

pdf

pdf

pdf, tex

pdf

pdf

pdf

html, pdf, tex

html, pdf, tex

html, pdf, tex

html, pdf, tex

html, pdf, ps, dvi, tex, ePUB

html, pdf, ps, dvi, tex, ePUB

pdf

pdf

html, pdf, ps, dvi, tex, ePUB

html, pdf

html ${ }^{* * * *}, p d f, p s, d v i$, tex 


\section{FROM THE AMS SECRETARY}

\section{Journal (Electronic)}

Number
of Articles
Posted in
2016

Math. Biosci. Eng. aimsciences.org/journals/home.jsp? journalID=8

Netw. Heterog. Media aimsciences.org/journals/home.jsp?journalID=9

New York J. Math. nyjm.albany.edu/nyjm.html

Open Math. www. openmathematics.com/

Proc. Amer. Math. Soc. Ser. B www.ams.org/publications/journals/ journalsframework/bproc

Reliab. Comput. interval.

louisiana.edu/reliable-computing-journal

Represent. Theory www. ams.org/pub7ications/journals/ journalsframework/ert

Sém. Lothar. Combin. www. mat. univie.ac.at/ slc

SIAM J. Appl. Dyn. Syst. epubs.siam.org/journal/siads/

SIAM J. Financial Math. epubs.siam.org/journal/sifin/

SIAM J. Imaging Sci. epubs.siam.org/journal/siims

SIAM/ASA J. Uncertain. Quantif. epubs.siam.org/journal/juq

Theory Appl. Categ. www.tac.mta.ca/tac/

Theory Comput. theoryofcomputing.org

Trans. Amer. Math. Soc. Ser. B www. ams.org/publications/journals/ journalsframework/btran
2016 Median Time (in days) from:

\begin{tabular}{c|c}
$\begin{array}{c}\text { Submission } \\
\text { to Final }\end{array}$ & $\begin{array}{c}\text { Acceptance } \\
\text { to } \\
\text { Pcceptance }\end{array}$ \\
Posting
\end{tabular}

Format(s)

\begin{tabular}{rrrl}
70 & 140 & 30 & pdf \\
36 & 145 & 30 & pdf \\
64 & 170 & 29 & pdf \\
103 & 105 & 47 & pdf, XML \\
3 & 240 & 132 & pdf \\
24 & 161 & 0 & pdf \\
17 & 198 & 39 & pdf \\
12 & 105 & 6 & pdf, ps, dvi, tex \\
77 & 228 & 78 & pdf \\
33 & 322 & 79 & pdf \\
73 & 186 & 82 & pdf \\
53 & 377 & 78 & pdf \\
38 & 281 & 3 & pdf \\
19 & 339 & 191 & htmlt, pdf, ps, , tex, \\
3 & 327 & 87 & pdf \\
\hline 3 & & &
\end{tabular}

NR means no response received. NA means not available or not applicable.

* Less than one.

$* *$ One volume in any year, no issues.

$* * *$ html for abstracts only.

† Applies to abstract, bibliography, author info, but not to full article. 

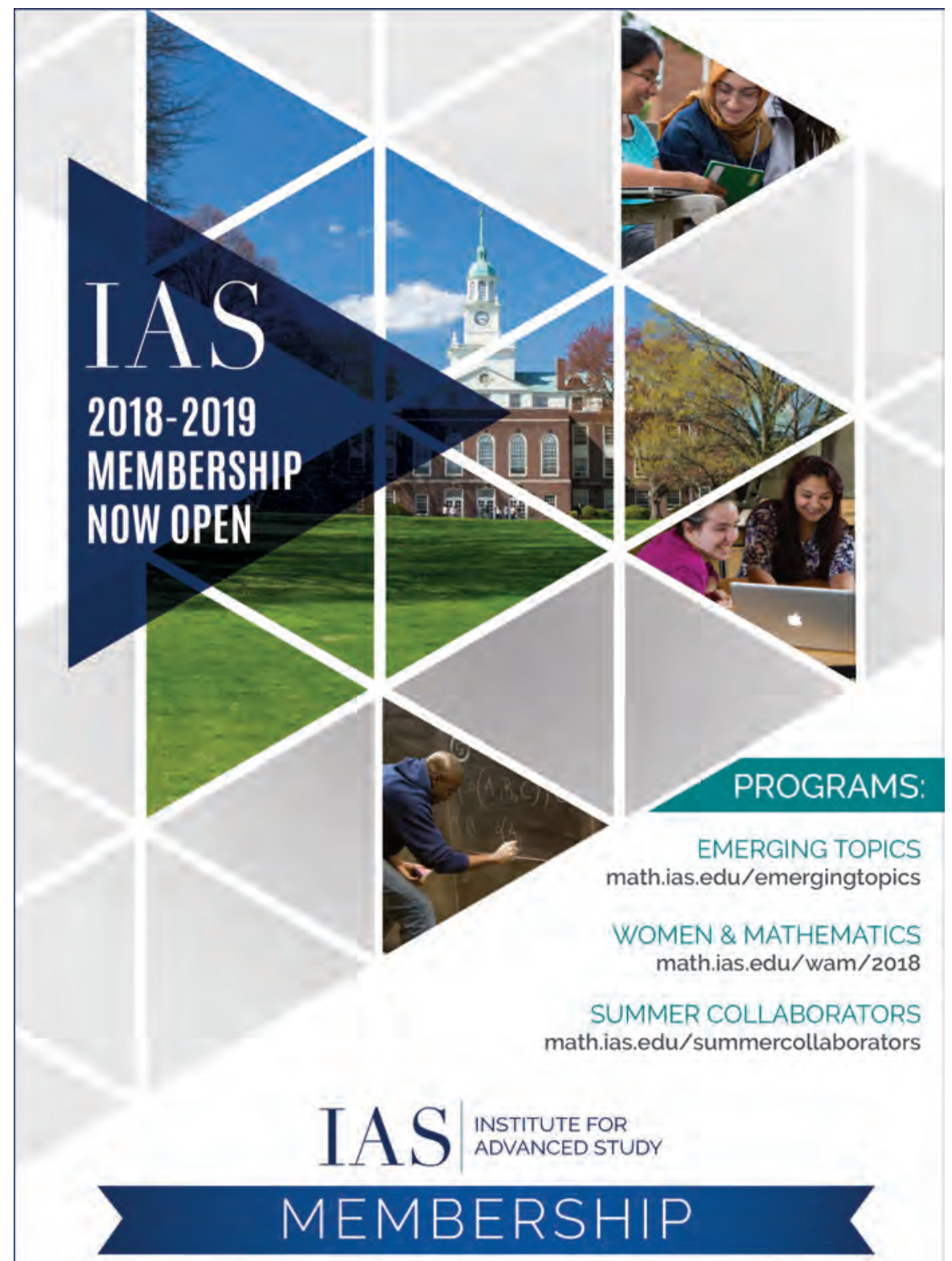

THE SCHOOL OF MATHEMATICS welcomes applications from postdoctoral, mid-career, and senior mathematicians and theoretical computer scientists, and strongly encourages applications from women and minorities.

Stipends, on-campus housing, and other resources are available for periods of 4-11 months for individual researchers in all mathematical subject areas. The School supports approximately 40 post-docs per year. In 2018-2019, there will be a special-year program called "Variational Methods in Geometry," led by Fernando Codá Marquez of Princeton University, however. Membership will not be limited to mathematicians in this field. For more information, please visit: math.ias.edu/administration/membership

Application Deadline: December 1, $2017 \cdot$ mathjobs.org

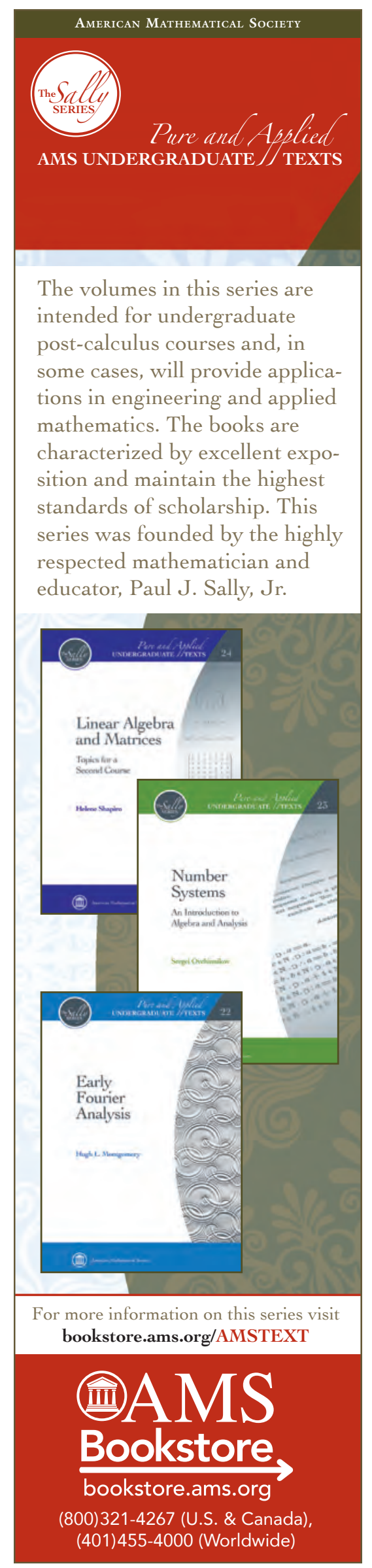




\section{BOOK REVIEW}

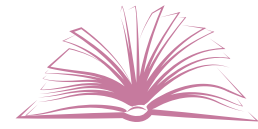

\section{The Slow Professor}

\section{A Review by David Manderscheid}

\author{
The Slow Professor: Challenging the Culture of Speed \\ in the Academy \\ by Maggie Berg and Barbara Seeber \\ University of Toronto Press, 2016 \\ 136 pages
}

Have you ever had a student email you at eleven o'clock at night to ask you a question about a homework assignment that was due the next day? Was that student then upset with you when you did not get back to him before class at eight-thirty the next morning? Have you ever emailed your department chair with a question expecting an answer the same day and she didn't get back to you until the next day? These are examples that illustrate the subject matter of this book. The pace of academic life has increased over the years. Many academics, including the authors of this book, believe that this trend is not healthy and that we should make every effort to reverse course.

The authors are tenured full professors of English and occasionally use language and terms more common to the humanities than to mathematics, but they make their argument for all disciplines. In particular, they assert that the "corporatization" of the university has led to dismissal of reflective work in pursuit of economic goals. They argue that metrics imposed by deans - and let me be clear, I am one-such as grant dollars, impact factors, and number of publications should be eschewed in favor of attaching greater value to contemplative thought and reflection about our disciplines and research.

I imagine that many of you might be sympathetic to their argument at this point. After all, Andrew Wiles did not publish for years. Moreover, he would not say what

David Manderscheid is executive dean of the College of Arts and Sciences, vice provost for Arts and Sciences, and professor of mathematics at Ohio State University. His e-mail address is manderscheid.1@osu.edu.

For permission to reprint this article, please contact:

reprint-permission@ams .org.

DOI: http://dx.doi.org/10.1090/noti1584

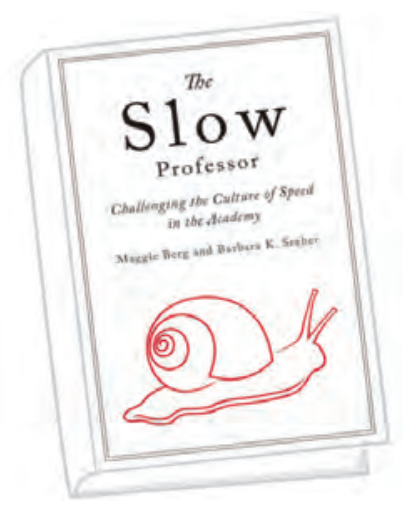

he was working on until he released a preprint of what he thought was a proof of Fermat's Last Theorem. His argument had a gap, but Richard Taylor soon helped him fill that lacuna. But what if Wiles had not released the preprint? What if, à la Fermat, he had merely written a marginal note in his copy of a paper by Goro Shimura, saying that he had a proof of Fermat's Last Theorem but the margin was too small to contain it? Or what about your colleague who's down the hall (at least when he's in his office, which isn't often)? He hasn't published in ten years, shuns committee work, and his students give him horrible teaching evaluations. Perhaps, though, he is days away from a verification of the Riemann Hypothesis.

Of course I am engaging in hyperbole to make my point. We need accountability in academe but we shouldn't be slaves to metrics imposed by the administration of our universities. How do we find the sweet spot between these extremes?

The authors suggest that we can find the answer to this question by looking to the slow food movement for inspiration. The slow food movement advocates embracing local products and regional and traditional cuisine and renouncing "fast food" and products from distant corporate farms. The movement was founded thirty years ago in Italy and has since spread worldwide, contributing to the proliferation of farmers markets, farm-to-table restaurants, and the like. The related slow living movement, started ten years ago, applies the slow food principles to life more generally. To oversimplify, slow living is mindful living - taking the time to reflect on one's life and the lives of others. The authors claim they are the first to apply the slow food and slow living principles to academe. 
The authors' viewpoint is not one of nostalgia for the past-far from it. Yes, the pace in academe has increased, they say, but it is now time for us to reclaim academe and fight the "remasculinized" university, which dismisses turning inward and disavows emotion in favor of hyperrational economic goals.

Here are a couple of the main arguments presented in the book. Trying to deal with the increased pace of academe through better time management is to buy into the system imposed upon us by our universities, in which they foster loyalty in order to improve productivity. We are reluctant to talk about the stress of being in academe for fear of being seen as weak. Buying into the language of crisis in academe worsens our fears. What we need to do is to stop abusing ourselves with overwork. I am reminded of a famous scene from the I Love Lucy television show of my childhood. In that scene Lucy is working on the assembly line of a chocolate factory. She views it as the ideal job, as the pace is not too fast and every so often she gets to eat a chocolate. But as the scene progresses the production line starts to move faster and faster. All of a sudden her ideal job has become a night-

reluctance to talk about the stress of being in academe
for fear of being
seen as weak mare. That is what the authors argue has happened to our ideal jobs in academe.

Recommending we apply slow principles in both our research and our teaching, they say that we need to spend more time off-line. In particular, they assert that the ability to work on-line at home and thus spend less time in our offices lessens our interactions with others, leading to poorer research and teaching. They point to the work of Sherry Turkle, Professor of Social Studies of Science and Technology at the Massachusetts Institute of Technology, to argue that we have become "universities of the absent," with a diminished sense of community. I must admit that I find this argument peculiar. To my mind the ability to work from home, for example, empowers faculty members to better manage the blend of their work and personal lives. As for spending more time off-line, I am reminded of my wife's mother who didn't like to talk on the telephone or do email. We would have fewer interactions with her than we would have otherwise, even though she lived in the same city, as we actually had to visit in person. The internet and smart phones enable us. We just need to use them in appropriate ways.

On the subject of research, the authors make the following points. The corporatization of the university has led to the privileging of certain types of research over others, so that research for the sake of knowledge is not valued, while research that has application is privileged. This corporatization has also led to collaboration being about dividing the work when it should be instead about the sharing of ideas. The emphasis on shortening time to $\mathrm{PhD}$, particularly in the humanities, buys into the corporatiza- tion of the university and leads to poorer outcomes for our students.

On the teaching front, the authors argue that we should reduce emphasis on learning outcomes, as they bastardize things that can't be measured into things that can be measured. We should move away from evidence-based teaching to teaching based on joy. One of the keys to such a move is to take the time to prepare psychologically for teaching our classes much like an athlete prepares psychologically to compete. If we convey positive emotions in our teaching, the authors say, then students will learn more.

I have some sympathy for their arguments both on research and on teaching. I do think the research pendulum has swung too far to areas where applications are immediate. Universities are where basic research gets done, and we need that for the future. That said, basic research for an English professor and a math professor are relatively inexpensive investments, while a more nuanced approach is needed when you move into fields like chemistry. As for the argument against reducing the time to the $\mathrm{PhD}$, I don't buy it. For too long we have believed that, with the explosion of knowledge, graduate students need to know more and more before they graduate. This belief does our students a disservice, and, to be blunt, we often cling to it unwittingly for our own benefit.

This provides a natural segue to my next criticism of the book. The authors write, as I do, from a position of

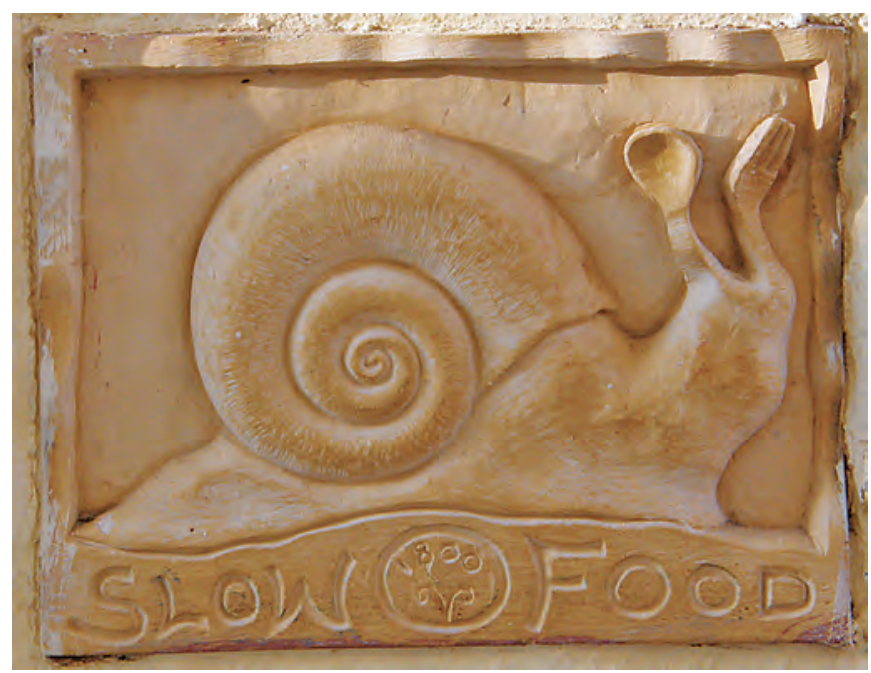

This photo shows a plaque posted outside the 1800 Restaurant in Oia, Santorini, on the Cyclades Islands in Greece. Principles of the "slow food" movement-such as appreciating traditions, taking the time to savor life, valuing personal relationships, and avoiding automation and haste-provided inspiration for the book The Slow Professor. 
privilege. We are tenured full professors at major research universities with incomes that are much greater than that of the average worker. The number of tenure-track faculty nationally is dwindling. With that comes the rise in the number of non-tenure track and adjunct faculty. I do agree that we should spend more time on our teaching, but the nominal teaching load for a faculty member in the Ohio State University Department of Mathematics is $2 / 1$. What about the adjunct faculty member at another institution who teaches a 4/4 load and struggles to meet financial obligations? Is teaching with more "joy" realistic for these faculty members?

Despite my criticisms I highly recommend reading this book. The authors make valid and important points about staying true to our research and teaching missions and taking care of ourselves and those around us. Moreover the book is a quick read at roughly a hundred pages. I read it on a plane flight, along with another academic book, and also did some email. One might argue that the book didn't have much effect on me since reading it was the first thing I did on the flight, and then I continued with my administrative work instead of perhaps napping, thinking about research, or watching a mindless movie. But I would argue the opposite. I was doing what I wanted to do, and that is one of the privileges of the life that I am able to live as a tenured full professor.

\section{Photo Credits}

Slow Food photo (c) Klearchos Kapoutsis, from https://www. f1ickr.com/photos/k1earchos/2261601356. Reprinted under terms of the Creative Commons Attribution 2.0 Generic license.

Photo of David Manderscheid by Kevin Fitzsimons, Ohio State University.

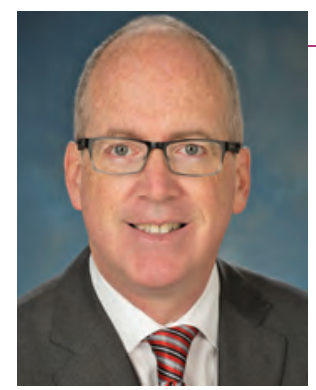

\section{ABOUT THE REVIEWER}

David Manderscheid's research is in the area of representation theory with applications to number theory. His outside interests include cycling and cooking.

\section{David}

Manderscheid

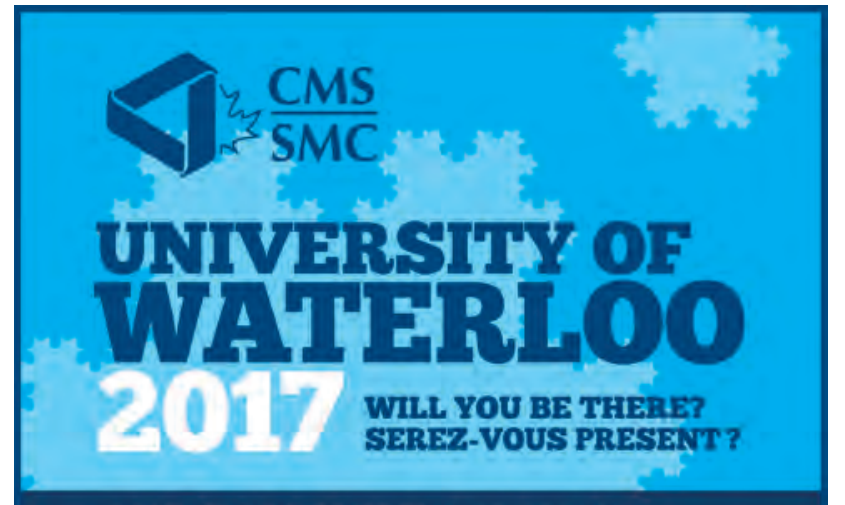

2017 CMS WINTER MEETING REUNION D'HIVER DE LA SMC 2017

DECEMBER 9-11 - 9-11 DÉCEMBRE

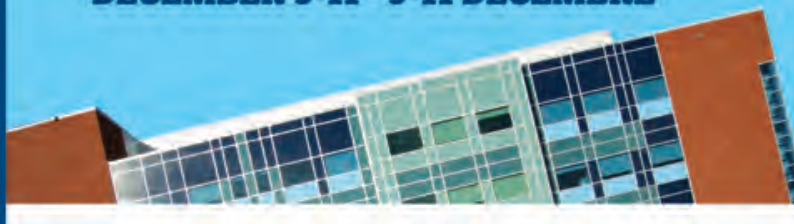

2017 Canadian Mathematical Society Winter Meeting

University of Waterloo, Waterloo, Ontario

\section{Scientific Directors}

Kenneth Davidson (University of Waterloo) Cameron L. Stewart (University of Waterloo)

\section{Prizes}

Adrien Pouliot Award

Coxeter-James Prize

Doctoral Prize

Excellence in Teaching Award

Graham Wright Award for Distinguished Service

Highlights

AARMS-CMS Student Poster Session

Contributed Papers

Education Sessions

Over 20 Scientific Sessions

CMS Awards Banquet
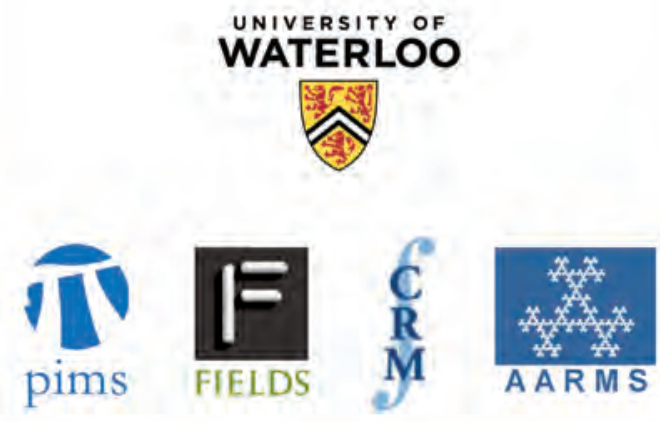

cms.math.ca/Events/winter17 \#CMSWinter 


\section{BOOKSHELF}

\section{New and Noteworthy Titles on Our Bookshelf November 2017}

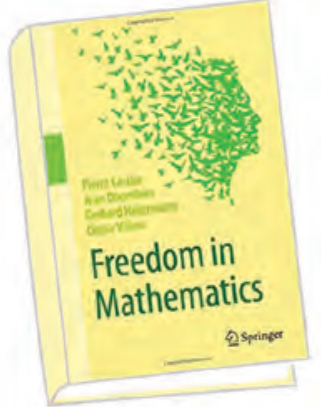

Freedom in Mathematics, by Pierre Cartier, Jean Dhombres, Gerhard Heinzmann, and Cédric Villani (Springer, April 2016)

This book originally appeared in 2012 under the title Mathématiques en liberté, was a volume in the series called 360, put out by the French publisher La ville brûle. The series treats a variety of scientific topics in a way that encourages readers to make a critical examination of the role of science in society. Edited by the noted French science journalist Sylvestre Huet, the 360 series has covered such topics as biodiversity, climate change, and the Big Bang. The English translation of the present book not only makes the content accessible to a wider audience but also allows a glimpse into the culture of mathematics in France. Two of the authors, Pierre Cartier and Cédric Villani, are likely well known to Notices readers. Cartier, now 85-years-old, is a key figure in the development of modern algebraic geometry and a longtime Bourbaki member. Cédric Villani, half Cartier's age, is a Fields Medalist who has a deep commitment to communicating about mathematics with the general public and who was elected to the French National Assembly this year. The other two authors are Jean Dhombres, a mathematician and historian, and Gerhard Heinzmann, a philosopher who specializes in the scientific philosophy of Poincaré. The book is organized into four chapters-On the Origins of Mathematics, Mathematics and Reality, Political and Social History of Mathematics Education, and The Nature and Challenges of Mathematics Research-and ends with a brief conclusion. Within each chapter, the narrative proceeds like a conversation, with Huet posing substantial and challenging questions and the four authors responding in turn. The discussion stays lively and provocative as the authors discuss, disagree, and digress. On the MAA Reviews web site, Darren Glass likens reading this book to being a fly on the wall in a room where four great minds are debating a wide variety of deep and intriguing issues in mathematics. He goes on: "The book is brief, and certainly reads more like an extended version of the kinds of interviews that one reads in the Notices of the American Mathematical Society than it does a deep and heavily researched monograph."

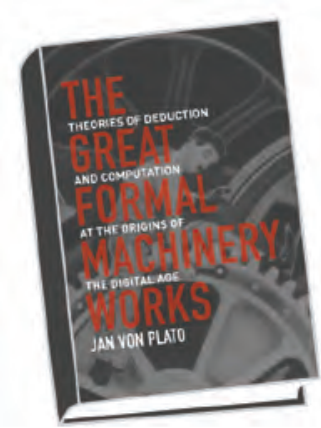

The Great Formal Machinery Works: Theories of Deduction and Computation at the Origins of the Digital Age, by Jan von Plato (Princeton University Press, August 2017)

"If around 1930 Kurt Gödel had not thought very deeply about the foundations of mathematics, there would be no information society in the form in which we have it today," asserts Jan von Plato in the prologue of The Great Formal Machinery Works. Gödel's work led directly to the first inklings of a theoretical computer, developed in the work of Alan Turing and of John von Neumann. von Plato actually traces the origins of these developments back yet further, to notions of formal proof in mathematics that emerged in the nineteenth century, particularly in the work of Grassmann and Peano. This book thus offers a comprehensive overview of a chain of ideas that led to the computational devices that are ubiquitous in modern life. The main outline of this story is known, but von Plato notes that a detailed, organized history based on primary sources has yet to be made. He contrasts work on the history of mathematical logic and foundations with the history of quantum mechanics. The latter received serious and comprehensive treatment starting in the 1960s, "in a massive effort in which every conceivable source was unearthed and made available in several hundred microfilm reels, the Archive for the History of Quantum Physics. Sadly, nothing of this kind was ever done with logic and foundations." In an endorsement for the book that appears on the publisher's web site, Paolo Mancosu, a philosopher at the University of California, Berkeley, says, "von Plato covers an immense territory with an impressive amount of novel things to say. He has written a very original book that is a major scholarly contribution to the history of logic." von Plato is also the author of Saved from the Cellar: Gerhard Gentzen's Shorthand Notes on Logic and Foundations of Mathematics (Springer, 2017), which contains translations of notes by Gentzen that had been lost for decades.

The BookShelf is prepared each month by Allyn Jackson. Suggestions for the BookShelf can be sent to notices-book1ist@ams. org.

We try to feature items of broad interest. Appearance of a book in the Notices BookShelf does not represent an endorsement by the Notices or by the AMS. For more, visit the AMS Reviews webpage www. ams.org/news/math-in-the-media/reviews. 

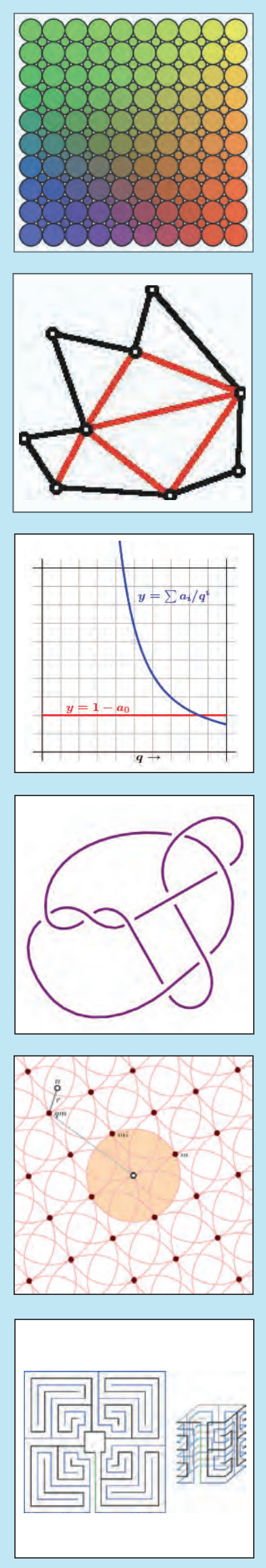

\section{THE FEATURE COLUMN} monthly essays on mathematical topics

Each month, the Feature Column provides an online in-depth look at a mathematical topic. Complete with graphics, links, and references, the columns cover a wide spectrum of mathematics and its applications, often including historical figures and their contributions. The authors-David Austin, Bill Casselman, Joe Malkevitch, and Tony Phillips—share their excitement about developments in mathematics.

Recent essays include:

Hidden Symmetries of Labyrinths from Antiquity and the Middle Ages

Circles and Squares

Petals, Flowers and Circle Packings

Are Precise Definitions a Good Idea?

Knot Quandaries Quelled by Quandles

The Legend of Abraham Wald

It Just Keeps Piling Up!

Mathematics and Crystal Balls

\section{www.ams.org/featurecolumn}


...When guys at MIT or Princeton had trouble doing a certain integral, it was because they couldn't do it with the standard methods they had learned in school. If it was contour integration, they would havefound it; if it was a simple series expansion, they would have found it. Then I come along and try differentiating under the integral sign, and often it worked. So I got a great reputation for doing integrals, only because my box of tools was different from everybody else's, and they had tried all their tools on it before giving the problem to me. -Richard Feynman, Surely You're Joking, Mr. Feynman

\section{Hypercohomology}
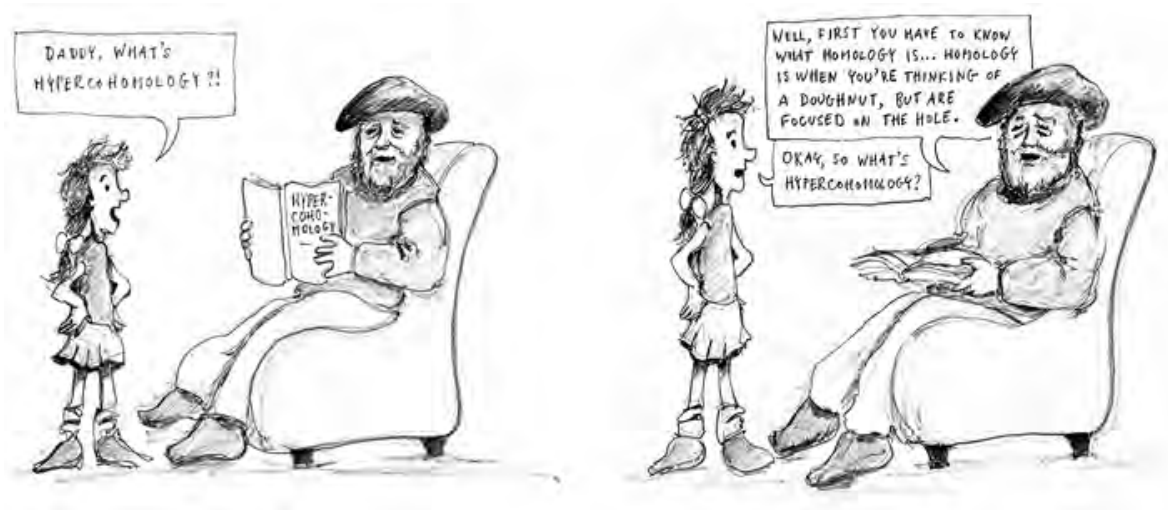

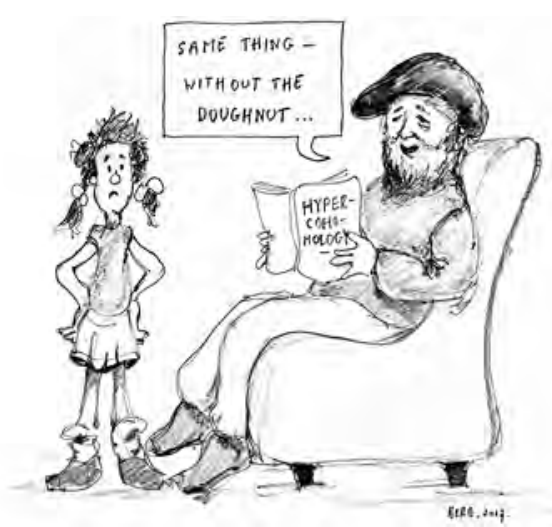

Concept by Sylvain Cappell, drawn by Michael Berg.

L. J. Mordell (1888-1972) was a leading number theorist from St. John's College, Cambridge University, England. When he was living in the United States, he received a phone call from a woman who asked him if he would like to participate in a quiz program. He agreed, so she asked him if he knew which country the rumba came from. He answered "Cuba," and she said, "You're right, and you've just won 75 dollars worth of dancing lessons." To this Mordell said, "Fine, now I have a question for you: Who was the first president of the United States?" She replied, "George Washington," to which Mordell said, "You're right, and now you've just won your dancing lessons back again." Mordell considered this to be one of his greatest triumphs.

What crazy things happen to you? Readers are invited to submit original short amusing stories, math jokes, cartoons, and other material to: noti-backpage@ams.org. 

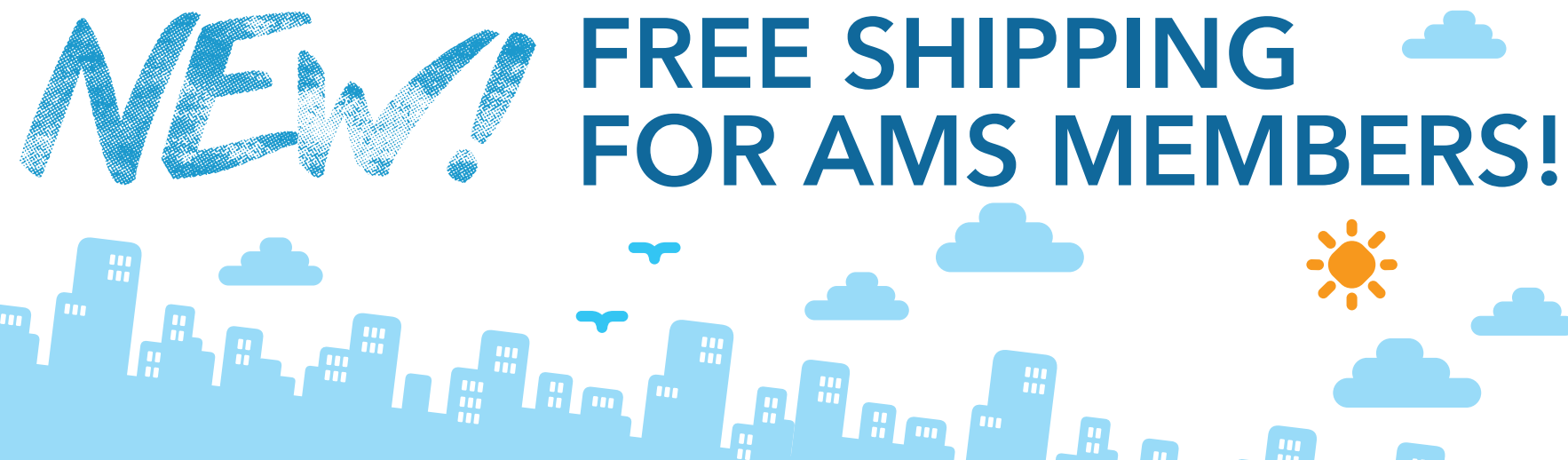

In addition to receiving a discount on books purchased

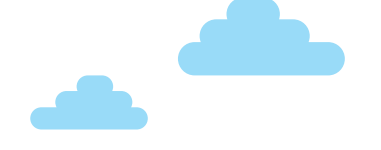

$$
\because
$$
through the AMS Bookstore and at meetings...

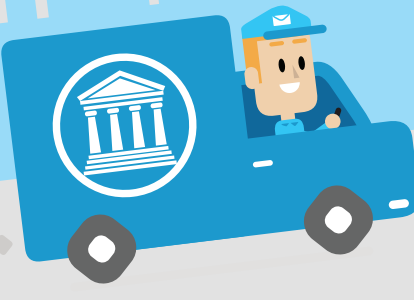

... all AMS members will also receive free shipping on their purchases!

Visit bookstore.ams.org to see our latest releases and what's on sale.

Visit ams.org/membership to learn more about membership benefits.

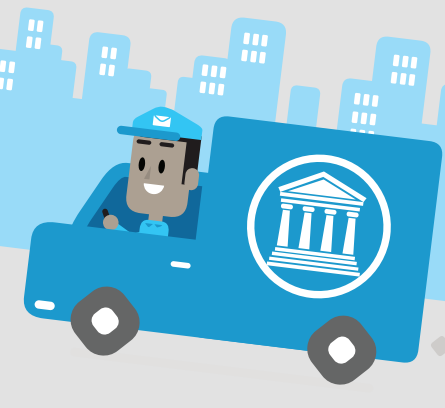




\section{Reciprocity Agreements}

The American Mathematical Society has reciprocity agreements with a number of mathematical organizations around the world. A current list of the reciprocating societies appears here; for full details of the agreements, see www.ams.org/membership/individual/mem-reciprocity.

Allahabad Mathematical Society

Argentina Mathematical Society

Australian Mathematical Society

Austrian Mathematical Society

Azerbaijan Mathematical Society

Balkan Society of Geometers

Bangladesh Mathematical Society

Belgian Mathematical Society

Berliner Mathematische Gesellschaft

Bharata Ganita Parisad

Brazilian Mathematical Society

Brazilian Society of Computational

and Applied Mathematics

Calcutta Mathematical Society

Canadian Mathematical Society

Catalan Society of Mathematicians

Chilean Mathematical Society

Colombian Mathematical Society

Croatian Mathematical Society

Cyprus Mathematical Society

Danish Mathematical Society

Dutch Mathematical Society

Edinburgh Mathematical Society

Egyptian Mathematical Society

European Mathematical Society

Finnish Mathematical Society

German Mathematical Society

German Society for Applied

Mathematics and Mechanics

Glasgow Mathematical Association

Hellenic Mathematical Society

Icelandic Mathematical Society
Indian Mathematical Society

Indonesian Mathematical Society

Iranian Mathematical Society

Irish Mathematical Society

Israel Mathematical Union

Italian Mathematical Union

János Bolyai Mathematical Society

Korean Mathematical Society

London Mathematical Society

Luxembourg Mathematical Society

Macedonian Society Association Mathematics/Computer Science

Malaysian Mathematical Society

Mathematical Society of France

Mathematical Society of Japan

Mathematical Society of the Philippines

Mathematical Society of the Republic of China

Mathematical Society of Serbia

Mexican Mathematical Society

Mongolian Mathematical Society

Nepal Mathematical Society

New Zealand Mathematical Society

Nigerian Mathematical Society

Norwegian Mathematical Society

Palestine Society for Mathematical Sciences

Parana's Mathematical Society

Polish Mathematical Society

Portuguese Mathematical Society

Punjab Mathematical Society
Ramanujan Mathematical Society

Romanian Mathematical Society

Romanian Society of Mathematicians

Royal Spanish Mathematical Society

Saudi Association for Mathematical Sciences

Singapore Mathematical Society

Sociedad Matemática de la Republica Dominicana

Sociedad Uruguaya de Matemática y Estadística

Société Mathématiques Appliquées et Industrielles

Society of Mathematicians, Physicists and Astronomers of Slovenia

South African Mathematical Society

Southeast Asian Mathematical Society

Spanish Mathematical Society

Swedish Mathematical Society

Swiss Mathematical Society

Tunisian Mathematical Society

Turkish Mathematical Society

Ukrainian Mathematical Society

Union of Bulgarian Mathematicians

Union of Czech Mathematicians and Physicists

Union of Slovak Mathematicians and Physicists

Vietnam Mathematical Society

Vijnana Parishad of India 


\section{Associate Editor, Mathematical Reviews} Algebra | 2018

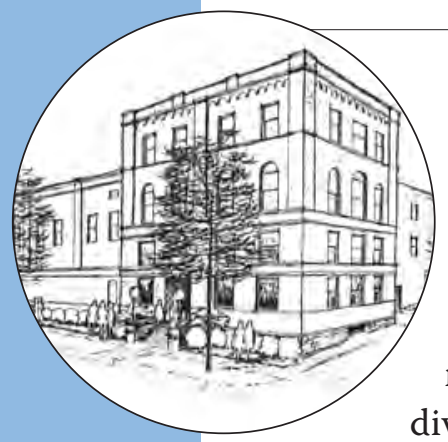

Applications are invited for a full-time position as an Associate Editor of Mathematical Reviews/MathSciNet ${ }^{\circ}$, to commence as early as possible in late spring/early summer 2018. We also welcome applications from suitably qualified candidates interested in spending a sabbatical year from their institution in the position and continuing as an editor working remotely under contract afterwards. The Mathematical Reviews (MR) division of the American Mathematical Society (AMS) is located in Ann Arbor, Michigan, in a beautiful, historic building close to the campus of the University of Michigan. The editors are employees of the AMS; they also enjoy certain privileges at the university. At present, the AMS employs approximately eighty people at Mathematical Reviews, including sixteen mathematical editors. MR's mission is to develop and maintain the MR Database, from which MathSciNet is produced.

An Associate Editor is responsible for broad areas of the mathematical sciences. Editors select articles and books for coverage, classify these items, determine the type of coverage, assign selected items for review to reviewers, and edit the reviews on their return.

The successful applicant will have mathematical breadth with an interest in current developments, and will be willing to learn new topics in pure and applied mathematics. In particular, we are looking for an applicant with expertise in algebra and an interest in a range of algebraic topics, such as representation theory, nonassociative algebras, and group theory. The ability to write well in English is essential. The applicant should normally have several years of relevant academic (or equivalent) experience beyond the $\mathrm{PhD}$ Evidence of written scholarship in mathematics is expected. The twelve-month salary will be commensurate with the experience that the applicant brings to the position.

The review of the applications will begin on February 12, 2018 and will continue until the position is filled.

Applications can be submitted through MathJobs at www.mathjobs.org/jobs/jobs/10667 Inquiries may be sent to the Executive Editor:

Edward Dunne

Executive Editor, Mathematical Reviews

American Mathematical Society

416 4th Street

Ann Arbor, MI 48103 USA

egd@ams.org

The American Mathematical Society is an Affirmative Action/Equal Opportunity Employer. 


\section{Biennial Overview of AMS Honors}
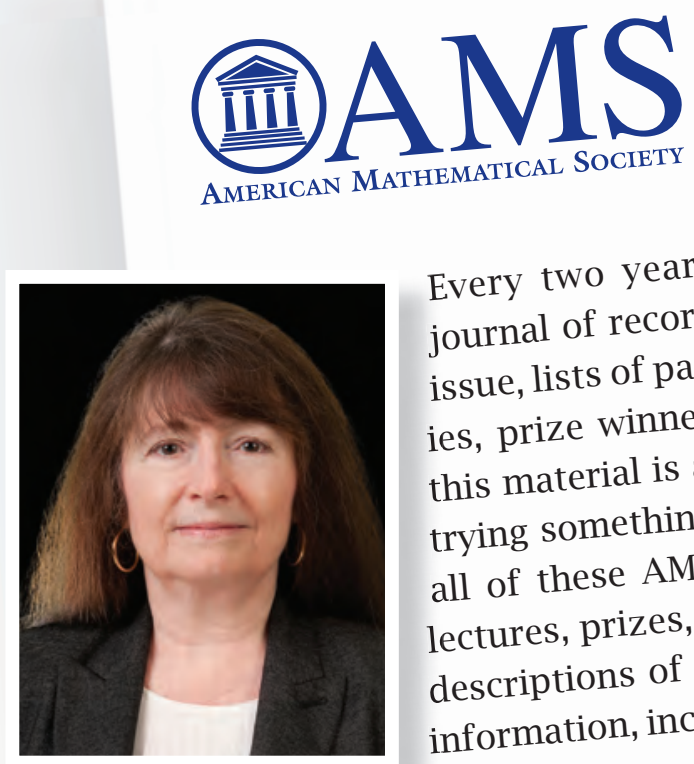

Every two years, the Notices, as the Society's journal of record, has published in its November issue, lists of past presidents, treasurers, secretaries, prize winners and special lecturers. Much of this material is available online, so this year we're trying something different. To give an overview of all of these AMS activities, we collect the special lectures, prizes, awards, and fellide links for further descriptions of each; and past recipients and lecturers. information, including past the Officers (President, The Society governance consists four Associate Secretaries, three Vice Presidents, Secreasurer), the Council, Executive Treasurer, and Associateincil, and Board of Trustees. You may Committee of the Cout Society governance at: www.ams.org/ find information about gocernance, which includes links to about-us/governance/govistory of the AMS: A Semicentennial books that chroniclerican Mathematical Society, 1888-1938, History of the Americanibald and History of the Second Fifty by Raymond Clare Archibald an Society, 1939-1988, by Everett Years, American Mathematical Society, 1939-19

We could not resist at least listing past presidents, treasurers, and secretaries below.

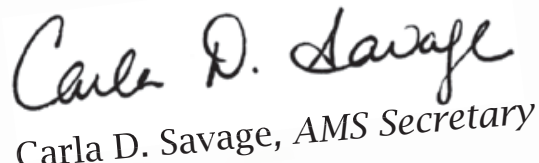




\section{Presidents}

2017-2018 Kenneth A. Ribet

2015-2016 Robert L. Bryant

2013-2014 David A. Vogan, Jr.

2011-2012 Eric M. Friedlander

2009-2010 George E. Andrews

2007-2008 James G. Glimm

2005-2006 James G. Arthur

2003-2004 David Eisenbud

2001-2002 Hyman Bass

1999-2000 Felix E. Browder

1997-1998 Arthur M. Jaffe

1995-1996 Cathleen Synge Morawetz

1993-1994 Ronald L. Graham

1991-1992 Michael Artin

1989-1990 William Browder

1987-1988 George Daniel Mostow

1985-1986 Irving Kaplansky

1983-1984 Julia Bowman Robinson

1981-1982 Andrew Mattel Gleason

1979-1980 Peter David Lax

1977-1978 R H Bing

1975-1976 Lipman Bers

1973-1974 Saunders Mac Lane

1971-1972 Nathan Jacobson

1969-1970 Oscar Zariski

1967-1968 Charles Bradfield Morrey, Jr.

1965-1966 Abraham Adrian Albert

1963-1964 Joseph Leo Doob

1961-1962 Deane Montgomery

1959-1960 Edward James McShane

1957-1958 Richard Dagobert Brauer

1955-1956 Raymond Louis Wilder
1953-1954 Gordon Thomas
Whyburn

1951-1952 John von Neumann

1949-1950 Joseph Leonard Walsh

1947-1948 Einar Hille

1945-1946 Theophil Henry Hildebrandt

1943-1944 Marshall Harvey Stone

1941-1942 Harold Calvin Marston Morse

1939-1940 Griffith Conrad Evans

1937-1938 Robert Lee Moore

1935-1936 Solomon Lefschetz

1933-1934 Arthur Byron Coble

1931-1932 Luther Pfahler Eisenhart

1929-1930 Earle Raymond Hendrick

1927-1928 Virgil Snyder

1925-1926 George David Birkhoff

1923-1924 Oswald Veblen

1921-1922 Gilbert Ames Bliss

1919-1920 Frank Morley

1917-1918 Leonard Eugene Dickson

1915-1916 Ernest William Brown

1913-1914 Edward Burr Van Vleck

1911-1912 Henry Burchard Fine

1909-1910 Maxime Bôcher

1907-1908 Henry Seely White

1905-1906 William Fogg Osgood

1903-1904 Thomas Scott Fiske

1901-1902 Eliakim Hastings Moore

1899-1900 Robert Simpson Woodward

1897-1898 Simon Newcomb

1895-1896 George William Hill

1891-1894 John Emory McClintock

1888-1890 John Howard Van Amringe

\section{Treasurers}

2011-2017 Jane M. Hawkins

1999-2010 John M. Franks

1974-1998 Franklin P. Peterson

1965-1973 W. T. Martin

1949-1964 A. E. Meder, Jr.

1938-1948 B. P. Gill

$1937 \quad$ P. A. Smith

1930-1936 G. W. Mullins

1921-1929 W. B. Fite

1908-1920 J. H. Tanner

1900-1907 W. S. Dennett

1897-1899 Harold Jacoby

1895, 1896 R. S. Woodward

1892-1894 Harold Jacoby

1890,1891 T. S. Fiske

\section{Secretaries}

$\begin{array}{ll}\text { 2013-2017 } & \text { Carla D. Savage } \\ 1999-2012 & \text { Robert J. Daverman } \\ 1989-1998 & \text { Robert M. Fossum } \\ 1967-1988 & \text { Everett Pitcher } \\ 1957-1966 & \text { J. W. Green } \\ 1951-1956 & \text { E. G. Begle } \\ 1941-1950 & \text { J. R. Kline } \\ 1921-1940 & \text { R. G. D. Richardson } \\ 1896-1920 & \text { F. N. Cole } \\ 1888-1895 & \text { T. S. Fiske }\end{array}$

2013-2017 Carla D. Savage

1999-2012 Robert J. Daverman

1989-1998 Robert M. Fossum

1967-1988 Everett Pitcher

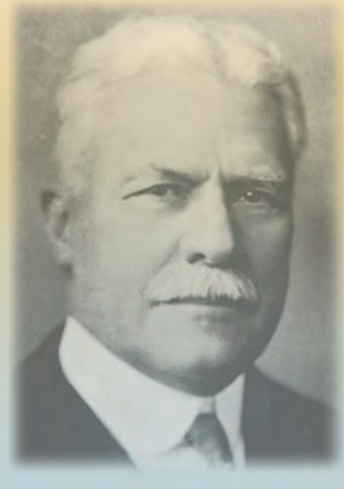

AMS Presidents: A Timeline

AMS presidents play a key role in leading the Society and representing the profession. Browse through the timeline to see each AMS president's page, which includes the institution and date of his/her doctoral degree, a brief note about his/her academic career and honors, and links to more extensive biographical information.

www.ams.org/presidents 


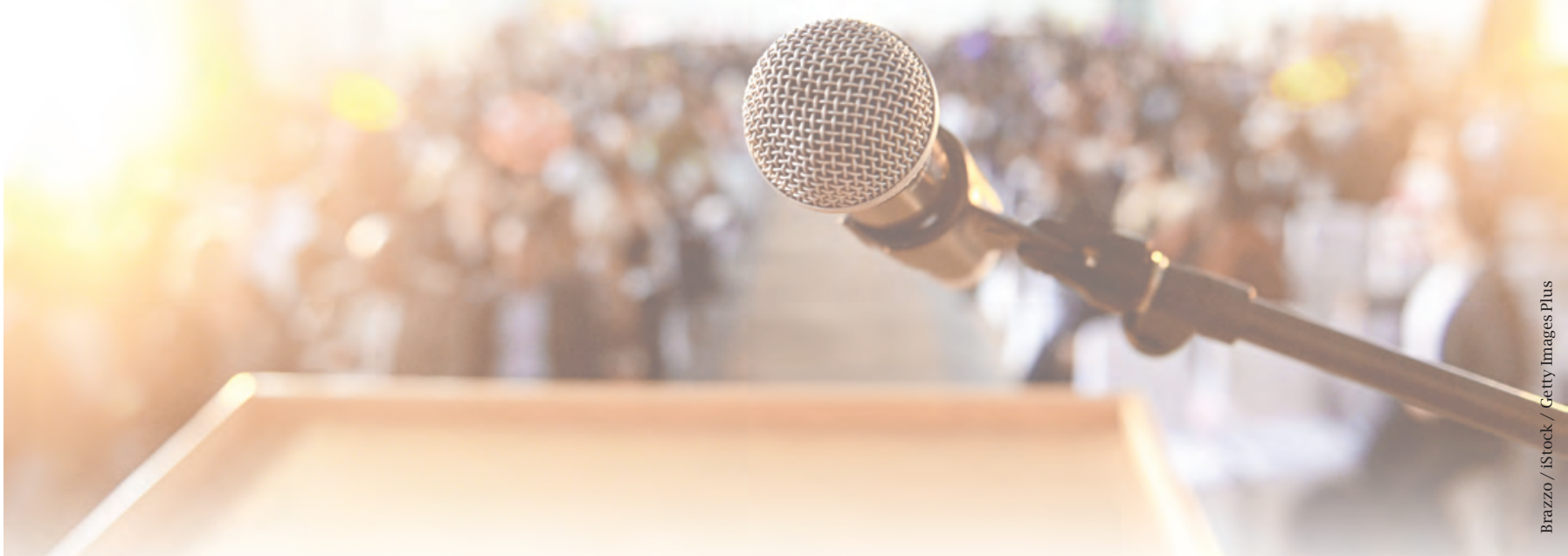

In addition to the Invited Addresses at the Joint Mathematics Meetings and at the eight Sectional Meetings each year, the Society sponsors or co-sponsors several special lectures.

\section{AMS Special Lectures}

\section{Colloquium Lectures}

\section{www.ams.org/meetings/lectures/meet-colloquium-lect}

The Colloquium Lectures have a long and prestigious history. Maxime Bôcher and James Pierpont delivered the first sets of lectures in 1896. They continue today as a highlight of the Joint Mathematics Meeting.

Josiah Willard Gibbs Lectures

www.ams.org/meetings/lectures/meet-gibbs-lect

These invited lectures are of popular nature, directed at those who are not professional mathematicians. The Society established the Gibbs Lectures in 1923.

Erdős Memorial Lectures

www.ams.org/meetings/lectures/meet-erdos-lect

The Erdós Memorial Lecture is an invited address given annually since 1999 at one of the Society's eight sectional meetings. The Lecture is name for the prolific mathematician Paul Erdős (1913-1996).

\section{Einstein Public Lectures in Mathematics}

www.ams.org/meetings/lectures/meet-einstein-lect

The Einstein Lectures, created by the AMS in 2005 to celebrate the one-hundreth anniversary of Einstein's annus mirabilis, are given annually at one of the Sectional Meetings.

\section{Arnold Ross Lectures}

www.ams.org/programs/students/ross-lectures/ross-lectures

Created by the AMS at the encouragement of Paul Sally, these annual lectures are aimed at talented high school mathematics students to stimulate their interest in mathematics beyond the traditional classroom.

\section{Joint Lectures}

\section{AMS-MAA-SIAM Gerald and Judith Porter Lectures www.ams.org/meetings/lectures/porter-lect}

The Porter Lecture on a mathematical topic accessible to the broader community is given each year at the Joint Mathematics Meetings.

AWM-AMS Noether Lectures https://sites.google.com/site/awmmath/programs/noetherlectures/noether-lecturers

The Noether Lecture, given each year at the Joint Mathematics Meetings honors women who have made fundamental and sustained contributions to the mathematical sciences.

\section{AMS-MAA Joint Lectures at MathFests} www.ams.org/meetings/lectures/ams-mathfest

These joint addresses, delivered annually at MathFest, are historical or expository in character.

\section{AMS-Sponsored Lectures at SIAM Meetings www.ams.org/meetings/lectures/ams-siam-lect}

AMS selects a lecturer to deliver an address at the SIAM Annual Meeting.

The AMS-NZMS Maclaurin Lectureship www.ams.org/meetings/lectures/maclaurin-lectures

This lectureship was a six-year reciprocal exchange between the New Zealand Mathematical Society and American Mathematical Society.

The Current Events Bulletin www.ams.org/meetings/lectures/current-events-bulletin Organized by David Eisenbud, this JMM event features speakers surveying some of the most interesting current developments in mathematics, pure and applied. 


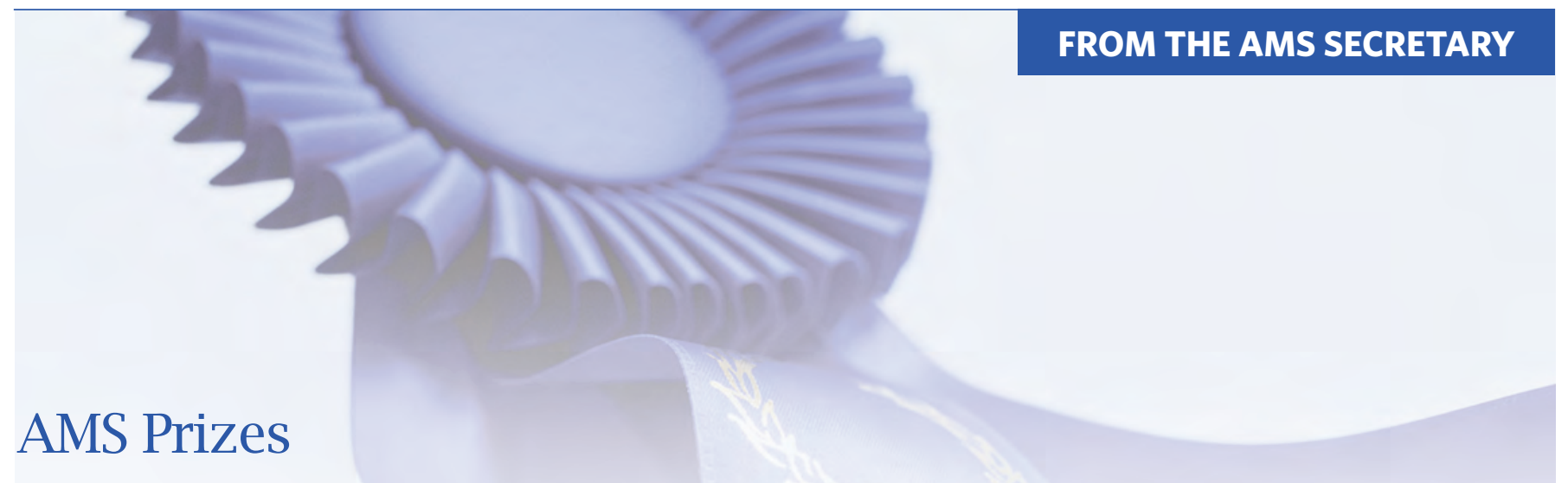

AMS Prizes recognize outstanding achievement in mathematics, exceptional public service in support of research and education in the mathematical sciences, and significant contributions to the public understanding of mathematics. The Society has added two new prizes this year: The Bertrand Russell Prize and the Ulf Grenander Prize in Stochastic Theory and Modeling. The prizes below are awarded at the Prize Ceremony at the Joint Mathematics Meeting.

Leroy P. Steele Prizes

www.ams.org/profession/prizes-awards/ams-prizes/steele-prize

- Lifetime Achievement

- Mathematical Exposition

- Seminal Contribution to Research

Bôcher Memorial Prize

www.ams.org/profession/prizes-awards/ams-prizes/bocher-prize

For a notable paper in analysis

Frank Nelson Cole Prize in Algebra

www.ams.org/profession/prizes-awards/ams-prizes/cole-prizealgebra

For a notable paper in algebra

Levi L. Conant Prize

www.ams.org/profession/prizes-awards/ams-prizes/conant-prize

For an expository paper published in either the Notices of the AMS or the Bulletin of the AMS

Ruth Lyttle Satter Prize in Mathematics www.ams.org/profession/prizes-awards/ams-prizes/satter-prize

For an outstanding contribution to mathematics research by a woman

Leonard Eisenbud Prize for Mathematics and Physics www.ams.org/profession/prizes-awards/ams-prizes/eisenbudprize

For work that brings mathematics and physics closer together

E. H. Moore Research Article Prize www.ams.org/profession/prizes-awards/ams-prizes/moore-prize

For a research article appearing in one of the AMS primary research journals

David P. Robbins Prize

www.ams.org/profession/prizes-awards/ams-prizes/robbinsprize

For a paper on novel research in algebra, combinatorics, or discrete mathematics
NEWt Bertrand Russell Prize of the AMS www.ams.org/profession/prizes-awards/russell-prize

For research or service contributions of mathematicians or related professionals to promoting good in the world, recognizing the various ways that mathematics furthers human values

Chevalley Prize in Lie Theory www.ams.org/profession/prizes-awards/chevalley-prize

For notable work in Lie Theory

Frank Nelson Cole Prize in Number Theory

www.ams.org/profession/prizes-awards/ams-prizes/cole-prizenumber-theory

For a notable paper in number theory

Joseph L. Doob Prize

www.ams.org/profession/prizes-awards/ams-prizes/doob-prize

For a single, relatively recent, outstanding research book

NEW Ulf Grenander Prize in Stochastic Theory and Modeling

www.ams.org/profession/prizes-awards/ams-prizes/grenanderprize

For theoretical and applied contributions in stochastic theory and modeling

Albert Leon Whiteman Memorial Prize www.ams.org/profession/prizes-awards/ams-prizes/whitemanprize

For notable exposition and exceptional scholarship in the history of mathematics

Oswald Veblen Prize in Geometry

www.ams.org/profession/prizes-awards/ams-prizes/veblen-prize For a notable research memoir in geometry or topology

Award for Distinguished Public Service www.ams.org/profession/prizes-awards/ams-awards/publicservice-award

For a research mathematician who has made a distinguished contribution to the mathematics profession 


\section{AMS Awards and Fellowships}

The Society added a new Fellowship this year: The Joan and Joseph Birman Fellowship for Women Scholars.

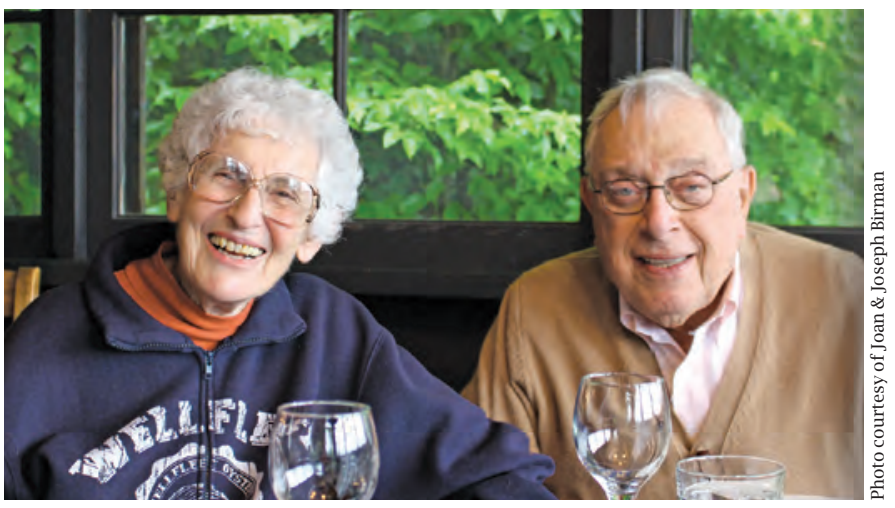

NEW The Joan and Joseph Birman Fellowship for Women Scholars

www.ams.org/programs/ams-fellowships/Birman-fellow

Seeks to address the paucity of women at the highest levels of research in mathematics by giving exceptionally talented women extra research support during their midcareer years.

Mathematics Programs that Make a Difference Award www.ams.org/profession/prizes-awards/ams-supported/makea-diff-award

Aims to bring more persons from underrepresented backgrounds into some portion of the pipeline beginning at the undergraduate level and leading to advanced degrees in mathematics and professional success, or retain them once in the pipeline.

Exemplary Program or Achievement in Mathematics Department Award

www.ams.org/profession/prizes-awards/ams-awards/ department-award

For a department which has distinguished itself by undertaking an unusual or particularly effective program of value to the mathematics community.

Karl Menger Memorial Award

www.ams.org/profession/prizes-awards/ams-awards/mengeraward

For mathematically oriented projects presented at the International Science and Engineering Fair.

\section{Centennial Fellowship}

www.ams.org/profession/prizes-awards/ams-awards/centennialfellow

For outstanding mathematicians to help further their careers in research, with a focus on candidates who have not had extensive fellowship support in the past.
Award for Impact on the Teaching and Learning of Mathematics www.ams.org/profession/prizes-awards/ams-awards/impact

For mathematicians who have made significant contributions of lasting value to mathematics education.

Epsilon Awards for Young Scholars Programs www.ams.org/profession/prizes-awards/ams-awards/epsilonaward

Supports existing summer programs for mathematically talented high school students.

Waldemar J. Trjitzinsky Memorial Awards www.ams.org/programs/ams-fellowships/trjitzinsky/trjitzinsky-award

Provides assistance to students who have declared a major in mathematics at a college or university that is an institutional AMS member.

\section{Joint Prizes and Awards}

George David Birkhoff Prize in Applied Mathematics www.ams.org/profession/prizes-awards/ams-prizes/birkhoffprize

Given jointly with the Society for Industrial and Applied Mathematics for an outstanding contribution to applied mathematics in the highest and broadest sense.

Norbert Wiener Prize in Applied Mathematics www.ams.org/profession/prizes-awards/ams-prizes/wiener-prize Given jointly with the Society for Industrial and Applied Mathematics for an outstanding contribution to applied mathematics in the highest and broadest sense.

Frank and Brennie Morgan Prize for Outstanding Research in Mathematics by an Undergraduate Student www.ams.org/profession/prizes-awards/ams-prizes/morganprize

Given jointly with the Mathematical Association of America and the Society for Industrial and Applied Mathematics for outstanding research in mathematics.

Delbert Ray Fulkerson Prize

www.ams.org/profession/prizes-awards/ams-prizes/fulkersonprize

Given jointly with the Mathematical Optimization Society for outstanding papers in the area of discrete mathematics.

JPBM Communications Award

www.ams.org/profession/prizes-awards/ams-awards/jpbmcomm-award

Given jointly with the Mathematical Association of America, the Society for Industrial and Applied Mathematics, and the American Statistical Association, to reward and encourage communicators who, on a sustained basis, bring mathematical ideas and information to non-mathematical audiences. 
Mathematical Art Exhibition Award

www.ams.org/profession/prizes-awards/ams-prizes/art-exhibitprize

Given jointly with the Mathematical Association of America for aesthetically pleasing works that combine mathematics and art.

\section{Other Prizes and Awards}

The Award for Outstanding Pi Mu Epsilon Student Paper Presentation, made by the national honorary mathematics society.

www.ams.org/profession/prizes-awards/ams-supported/pmeaward

The Public Policy Award was established in 2007 by the AMS to recognize a public figure for sustained and exceptional contributions to public policies that foster support for research, education, and innovation.

www.ams.org/profession/prizes-awards/ams-awards/publicpolicy-award

The Public Service Citation was created to provide encouragement and recognition for contributions to public service activities in support of mathematics.

www.ams.org/profession/prizes-awards/ams-awards/publicservice-citation

These two awards have not been given in recent years.

The Beal Prize, funded by D. Andrew Beal, is awarded for either a proof or a counterexample of the Beal Conjecture, which is published in a refereed and respected mathematics journal.

www.ams.org/profession/prizes-awards/ams-supported/bealprize

The Stefan Bergman Prize is awarded by the Bergman Trust in honor of his research in several complex variables, as well as the Bergman projection and the Bergman kernel function.

www.ams.org/profession/prizes-awards/ams-supported/ bergman-prize

The National Academy of Sciences Award in Mathematics.

Funding is currently being sought by the NAS to continue this award.

www.ams.org/profession/prizes-awards/ams-supported/nasaward

The Fredkin Foundation asked the AMS to take over administration of its Fredkin Foundation prizes in Automatic Theorem Proving in the mid-1980s. This prize is no longer awarded.

www.ams.org/profession/prizes-awards/ams-supported/atpprizes
The Leonard M. and Eleanor B. Blumenthal Award for the Advancement of Research in Pure Mathematics. This prize was discontinued by the trust.

www.ams.org/profession/prizes-awards/ams-supported/ blumenthal-award 


\section{William Thurston Marcel Berger Cédric}

Luis A. Caffarelli Michael Freedman Vladimir Drinfeld Shlomo Sternberg Terence Tao John Nilnor Jean-Pierre Serre Lars Gainding Jean Boungain Sigurdur Helgason Victor Guillemin

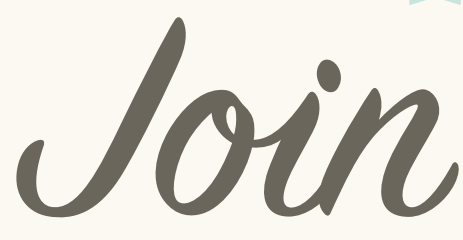

\section{the company} of great mathematicians

\section{BECOME AN AMS AUTHOR}

\section{WHY PUBLISH WITH THE AMS?}

We are mathematicians. The AMS is one of the world's leading publishers of mathematical literature. As a professional society of mathematicians, we publish books and journals for the advancement of science and mathematics. Consequently, our publications meet the highest professional standards for their content and production.

Expertise. Our editorial boards consist of experienced mathematicians. The AMS production staff is talented and experienced at producing high-quality books and journals. The author support group consists of experts in TeX, graphics, and other aspects of the production of mathematical content.

Supporting mathematics. The AMS publication program is a part of our broader activities. The revenue it generates helps support our other professional activities. Thus, publishing with the AMS benefits the mathematical community.

Learn more at:www.ams.org/becomeanauthor

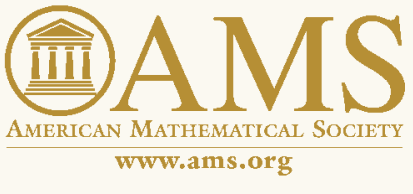



Mathematical Reviews

The right tool for Mathematics Research

\section{www.ams.org/mathscinet}

MathSciNet, the authoritative gateway to the scholarly literature of mathematics. The database contains information on more than 3 million articles and books and includes expert reviews, personalizable author profiles, and extensive citation information.

\section{Authors}

Customize your author profile today with your photo, email, and a link to your homepage Your profile also includes:

1 Share your profile with non-subscribers and subscribers alike, using the share button to create a universal link to your author profile page.

2 A link to your Mathematics Genealogy Project Profile-easily keep track of former students and advisors.

(3) Linked word clouds showing your publication areas, co-authors, and most-cited publications-see the impact of your research and quickly locate related work.

(4) A complete record of your MathSciNet review and publication activity-use it as a supplement to your university homepage, or as a quick reference when updating a CV.

$(5)$ An option for inputting your name in your native script or language.

Go to

www.ams.org/ mathscinet/ help/ about.html to get started.
Hame Proterencos Free Toels Help Support Mai Terms of Use
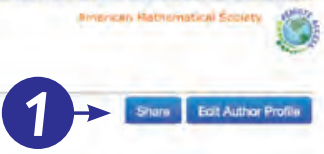

\section{Full name in native script: $\quad$ oph,$\pi$ ans}

Email: $\quad$ elkiescmath.harvard.edu

MR Author 1D: 229330

Earliest Incexed Publication: 1986

Total Publications: $\quad 70$

Total Citations: $\quad \mathbf{8 6 0}$

*. Published as: Elkies, N. D. .

View Author/Related Publications

Refine Search

Co-Authors

Collaboration Distance

Mathematics Genealogy Project

Citations
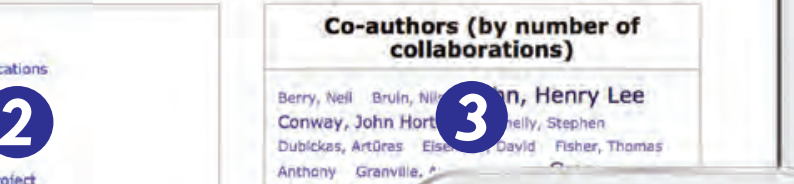
Benedict $\mathrm{H}$.

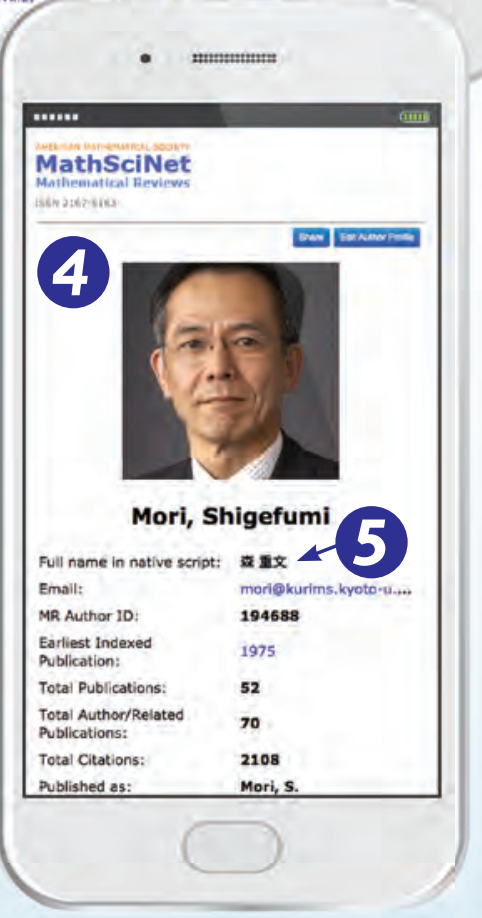




\section{Mathematics Opportunities}

Listings for upcoming math opportunities to appear in Notices may be submitted to notices@ams . org .

\section{American Mathematical Society Centennial Fellowship}

Invitation for Applications for Awards for 2018-2019 Deadline December 1, 2017

The AMS Centennial Research Fellowship Program makes awards annually to outstanding mathematicians to help further their careers in research. The number of fellowships to be awarded is small and depends on the amount of money contributed to the program. The Society supplements contributions as needed. At least one fellowship will be awarded for the 2018-2019 academic year. A list of previous fellowship winners can be found at www. ams.org/profession/prizes-awards/ams-awards/ centennial-fe17ow.

Eligibility: The eligibility rules are as follows. The primary selection criterion for the Centennial Fellowship is the excellence of the candidate's research. Preference will be given to candidates who have not had extensive fellowship support in the past. Recipients may not hold the Centennial Fellowship concurrently with another research fellowship such as a Sloan or NSF Postdoctoral fellowship. Under normal circumstances, the fellowship cannot be deferred. A recipient of the fellowship shall have held his or her doctoral degree for at least three years and not more than twelve years at the inception of the award (that is, received between September 1, 2006, and September 1, 2015). Applications will be accepted from those currently holding a tenured, tenure track, postdoctoral, or comparable (at the discretion of the selection committee) position at an institution in North America. Applications should include a cogent plan indicating how the fellowship will be used. The plan should include travel to at least one other institution and should demonstrate that the fellowship will be used for more than reduction of teaching at the candidate's home institution. The selection committee will consider the plan, in addition to the quality of the candidate's research, and will try to award the fellowship to those for whom the award would make a real difference in the development of their research careers. Work in all areas of mathematics, including interdisciplinary work, is eligible.
Deadline: The deadline for receipt of applications is December 1, 2017. The award recipient will be announced in February 2018 or earlier, if possible.

Application information: Find Centennial application information at www.ams.org/ams-fe11owships. For questions, contact the Professional Programs Department, American Mathematical Society, 201 Charles Street, Providence, RI 02904-2294; prof-serv@ams.org; 401455-4096.

-AMS Professional Programs Department

\section{AMS Congressional Fellowship}

The AMS, in conjunction with the American Association for the Advancement of Science (AAAS), will sponsor a Congressional Fellow from September 2018 through August 2019. The Fellow will spend the year working on the staff of a Member of Congress or a congressional committee as a special legislative assistant in legislative and policy areas requiring scientific and technical input.

The fellowship is designed to provide a unique public policy learning experience, to demonstrate the value of science-government interaction, and to bring a technical background and external perspective to the decisionmaking process in the Congress.

An AMS Fellowship Committee will select the AMS Congressional Fellow. The fellowship stipend is US $\$ 79,720$ for the fellowship period, with allowances for relocation and professional travel and a contribution towards health insurance. Applicants must have a $\mathrm{PhD}$ or an equivalent doctoral-level degree in mathematics by the application deadline.

For information and to apply, go to bit.7y/AMSCongressiona1Fe11owship. The deadline for applications is February 15, 2018.

-AMS Washington Office 


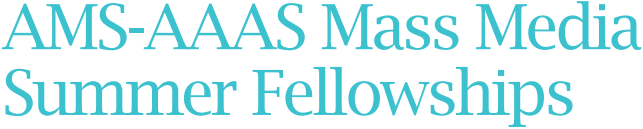

The American Mathematical Society provides support each year for a graduate student in the mathematical sciences to participate in the American Association for the Advancement of Science (AAAS) Mass Media Science and Engineering Fellows Program. This summer fellowship program pairs graduate students with major media outlets nationwide, where they will research, write, and report on science news and use their skills to bring technical subjects to the general public.

The principal goal of the program is to increase the public's understanding of science and technology by strengthening the connection between scientists and journalists to improve coverage of science-related issues in the media. Past AMS-sponsored fellows have held positions at National Public Radio, Scientific American, Voice of America, The Oregonian, NOVA, the Chicago Tribune, and the Milwaukee Journal Sentinel.

Fellows receive a weekly stipend of US\$500, plus travel expenses, to work for ten weeks during the summer as reporters, researchers, and production assistants in newsrooms across the country. They observe and participate in the process by which events and ideas become news, improve their ability to communicate about complex technical subjects in a manner understandable to the public, and increase their understanding of editorial decision making and of how information is effectively disseminated. Each fellow attends an orientation and evaluation session in Washington, DC, and begins the internship in mid-June. Fellows submit interim and final reports to AAAS. A wrapup session is held at the end of the summer.

Mathematical sciences faculty are urged to make their graduate students aware of this program. Further information about the fellowship program and application procedures is available online at www. aaas .org/programs/ education/MassMedia; or applicants may contact Rebekah Corlew, Program Director, AAAS Mass Media Science and Engineering Fellows Program, 1200 New York Avenue, NW, Washington, DC 20005; emai 1 rcorlew@aaas.org.

Further information is also available at www. ams.org/ programs/ams-fe11owships/media-fe11ow/massmediafel1ow and through the AMS Washington Office, 1527 Eighteenth Street, NW, Washington, DC 20036; telephone 202-588-1100; email amsdc@ams.org. The deadline for applications is January 15, 2018.

$$
\text { - AMS Washington Office }
$$

\section{AMS Epsilon Fund}

The AMS Epsilon Fund awards grants to summer mathematics programs that support and nurture mathematically talented high school students in the United States. The deadline to apply for funding for summer 2018 programs is December 15, 2017. Applications are now being accepted online at MathPrograms.org (www.math- programs . org). For more information about the program and updated application information, go to www.ams. org/programs/edu-support/epsilon/emp-epsilon or contact the AMS Professional Programs Department by email at prof-serv@ams .org or by telephone at 800321-4267, ext. 4096.

\section{- AMS Professional Programs Department}

\section{*NSF Graduate Research Fellowships}

The National Science Foundation's Graduate Research Fellowship Program recognizes and supports outstanding graduate students in NSF-supported science, technology, engineering, and mathematics disciplines who are pursuing research-based master's and doctoral degrees at US institutions. The NSF welcomes applications from all qualified students and strongly encourages underrepresented populations, including women, underrepresented racial and ethnic minorities, and persons with disabilities, to apply. Fellows receive a three-year annual stipend of US\$34,000, opportunities for international research and professional development, and the freedom to conduct their research at any accredited U.S. institution of graduate education. The deadline for the mathematical sciences is October 27, 2017. For further information, visitwww. nsf. gov/funding/pgm_summ.jsp?pims_id=6201.

-From NSF announcements

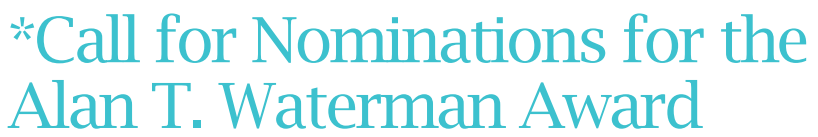

The National Science Foundation (NSF) is soliciting nominations for the 2017 Alan T. Waterman Award. The award recognizes an outstanding young researcher in any field of science or engineering supported by the NSF. The award consists of a US $\$ 1,000,000$ grant over a five-year period for research at the institution of the recipient's choice. The deadline for nominations is October 21, 2017. Nominations must be submitted at www.fastlane.nsf.gov/ honawards/. For more details, seewww.nsf.gov/od/waterman/waterman.jsp.

-From an NSF announcement 


\section{NEWS}

\section{Jefferson Science Fellows Program}

The Jefferson Science Fellows (JSF) program at the US Department of State involves the American academic science, technology, and engineering communities in the formulation and implementation of US foreign policy. Each fellow spends one year at the US Department of State or the US Agency for International Development (USAID) for an on-site assignment in Washington, DC, that may also involve extended stays at US foreign embassies and/or missions. Each fellow receives a stipend of up to US $\$ 50,000$ to cover living expenses for one year; an additional US\$10,000 is awarded for travel. Following the fellowship year, the Jefferson Science Fellow returns to his or her academic career but remains available to the US Department of State for short-term projects. The deadline for applications is October 31, 2017. The JSF program is administered by the National Academies and is supported through a partnership of the US science, technology, and academic communities, professional scientific societies, and the US Department of State. For further information, email jsf@nas.edu or see sites.nationalacademies. org/PGA/Jefferson/PGA_046612.

-From a National Academies announcement

\section{AAUW Educational Foundation Fellowships and Grants}

The American Association of University Women (AAUW) has programs supporting women students and scholars at various stages of their careers. Selected Professions Fellowships support women students in areas in which women's participation has traditionally been low, including computer/information sciences and mathematics/ statistics. For further information about the fellowships, application procedures, and deadlines, visit the website www . aauw . org/what-we-do/educational-fundingand-awards.

-From AAUW website

\section{AWM Essay Contest}

The Association for Women in Mathematics (AWM) and Math for America cosponsor an annual essay contest for biographies of contemporary women mathematicians and statisticians in academic, industrial, and government careers. Each essay will be based primarily on an interview with a woman currently working in a mathematical sciences career. This contest is open to students in the following categories: Grades 6-8, Grades 9-12, and College Undergraduate. Winners will receive a prize, and their essays will be published online. The deadline for the 2017 AWM Essay Contest is January 31, 2018. AWM is also currently seeking women mathematicians to volunteer as the subjects of these essays. For more information or to sign up as a volunteer, contact the contest organizer, Heather Lewis, at h1ewis5@naz.edu. See sites.goog1e.com/ site/awmmath/home for complete information.

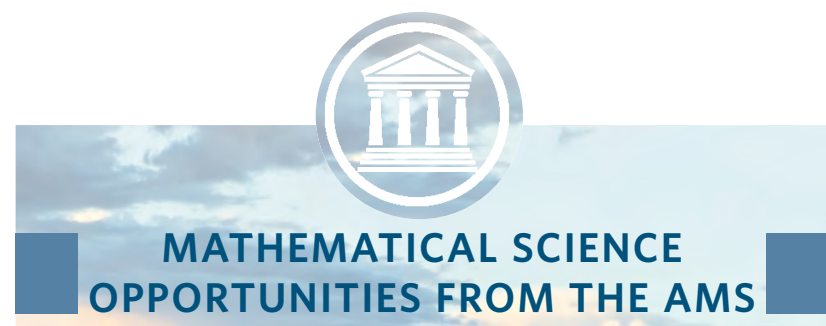

The AMS Online Opportunities Page provides another avenue for the math community to

Announce and Browse:

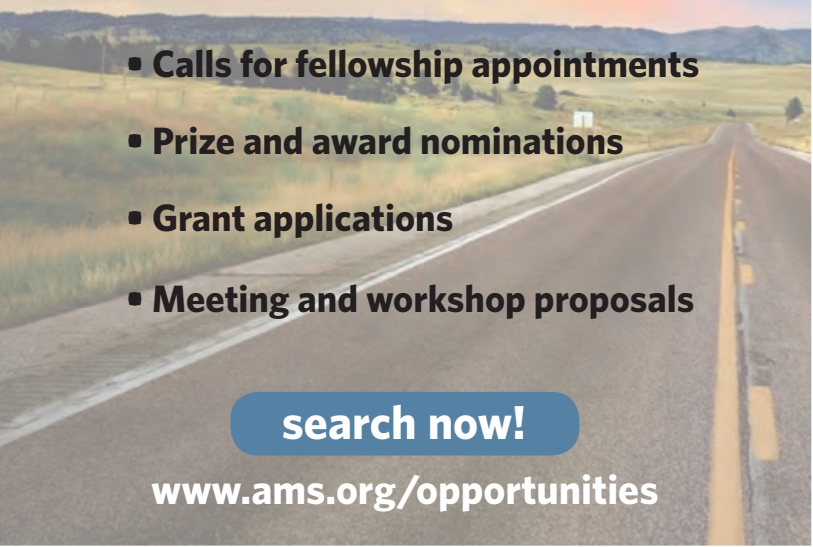




\section{Mathematics People}

\section{Simons Foundation Investigators Named}

The Simons Foundation has named the Simons Investigators for 2017.

\section{Mathematics:}

SIMON BRENDLE of Columbia University has achieved major breakthroughs in geometry, including results on the Yamabe compactness conjecture, the differentiable sphere theorem (joint with R. Schoen), the Lawson conjecture, and the Ilmanen conjecture, as well as singularity formation in the mean curvature flow, the Yamabe flow, and the Ricci flow.

LUDMIL KATZARKOV of the University of Miami has introduced novel ideas and techniques in geometry, proving long-standing conjectures (e.g., the Shavarevich conjecture) and formulating new conceptual approaches to open questions in homological mirror symmetry, rationality of algebraic varieties, and symplectic geometry.

IGOR RODNIANSKI of Princeton University is a leading figure in the field of partial differential equations. He has recently proven theorems concerning the full nonlinear dynamics of the Einstein equations, in both the weak and strong field regimes, and has obtained new results regarding gravitational radiation associated to black hole space times.

ALLAN SLY of the University of California, Berkeley has resolved long-standing open problems on the computational complexity of phase transitions and on the dynamics of the Ising model.

\section{Physics:}

SHAMIT KACHRU of Stanford University has done work that includes the discovery of string dualities in $N=2$ supersymmetry; foundational studies of flux compactification of string theory; mathematical studies of connections between automorphic forms, black holes, and string vacua; and quantum field theories describing "non-Fermi-liquid" behavior in condensed matter physics.

ANDERS SANDVIK of Boston University is widely recognized for his development of stochastic series expansion methods for quantum problems and for his creative applications of these and related methods to topics including deconfined quantum criticality and optimization problems.

EVA Silverstein of Stanford University has done research that connects the mathematical structure of string theory to predictions for cosmological observables, with implications for dualities, space-time singularities, and black hole physics. Her work on axion monodromy provided a theoretically consistent model of large-field inflation.

Theoretical Computer Science:

SCOTT AARONSON of the University of Texas at Austin has established fundamental theorems in quantum computational complexity and inspired new research directions at the interface of theoretical computer science and the study of physical systems.

BOAZ BARAK of Harvard University has worked on cryptography, computational complexity, and algorithms. He developed new non-black-box techniques in cryptography and new semidefinite programming-based algorithms for problems related to machine learning and the unique games conjecture.

JAMES R. LEE of the University of Washington is one of the leaders in the study of discrete optimization problems and their connections to analysis, geometry, and probability. His development of spectral methods and his work on convex relaxations has led to breakthroughs in characterizing the efficacy of mathematical programming for combinatorial optimization.

Mathematical Modeling of Living Systems:

ARVIND MURUGAN of the University of Chicago works on how organisms enhance information uptake from the environment by using inference from past experience and has applied such ideas to self-assembly dynamics, olfaction, circadian clocks, and stress-response pathways.

DAVID SCHWAB of Northwestern University has developed theories of signaling and social aggregation in the social amoeba Dictyostelium and has shown how tensornetwork methods from computational quantum physics can be used in machine learning.

ARYEH WARMFLASH of Rice University has developed systems to mimic embryonic development in vitro using human embryonic stem cells and is developing dynamical system models of cell fate patterning and morphogenesis that can be rigorously compared with quantitative data on in vitro development.

DANIEL WEISSMAN of Emory University has shown that the generation of "irreducible complexity" happens most frequently in large populations and that the speed of adaptation is limited by the frequency of genetic recombination. 


\section{Math + X:}

ANDREA BERTOZZI of the University of California, Los Angeles, has contributed to many areas of applied mathematics, including the theory of swarming behavior, aggregation equations and their solution in general dimension, the theory of particle-laden flows in liquids with free surfaces, data analysis/image analysis at the micro and nano scales, and the mathematics of crime.

AMIT SINGER of Princeton University is one of the leaders in the mathematical analysis of noisy data provided by cryo-EM.

The Simons Investigators program provides a stable base of support for outstanding scientists, enabling them to undertake long-term study of fundamental questions. -From a Simons Foundation announcement

\section{Teixeira Awarded ICTP-IMU Ramanujan Prize}

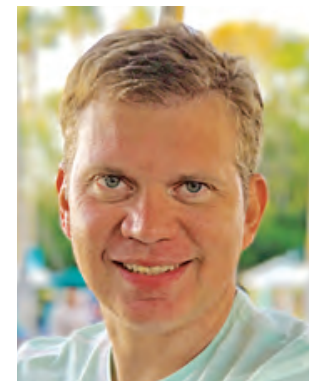

Eduardo Teixeira
EDUARDO TEIXEIRA of the Federal University of Ceará, Brazil, has been awarded the 2017 Ramanujan Prize for Young Mathematicians from Developing Countries in recognition of his outstanding work in analysis and partial differential equations. The prize is awarded by the Abdus Salam International Center for Theoretical Physics (ICTP), the International Mathematical Union (IMU), and the Department of Science and Technology of the Government of India.

The prize citation reads: "Teixeira started working on free boundary problems during his $\mathrm{PhD}$ thesis, proving existence and regularity results, and obtaining qualitative properties of solutions, in the theory of nonlinear heat conduction. Subsequently, in collaboration with L. Zhang, he obtained Almgren's type frequency formulas in Riemannian manifolds. He then introduced an original approach to the regularity of degenerate elliptic equations, which consists in viewing the set of critical points of a solution as a free boundary. This interesting point of view led him to prove the continuity conjecture for elliptic equations with high-order singular structures, and in solving, in collaboration with Araujo and Urbano, a long-standing conjecture on the optimal regularity for the $p$-Laplacian in two dimensions. Teixeira has contributed to many other aspects of the theory of nonlinear elliptic equations. A perfect example is his recent breakthrough, in collaboration with Y. Li and Z.-C. Han, on the asymptotic radial symmetry of solutions to the $k$ th-order Yamabe equation in punctured domains, a deep and original contribution to the theory of conformally nonlinear elliptic PDEs." The prize also recognizes his pursuit of high-level research in Brazil. At Ceará, he founded and directed one of the major research groups in partial differential equations in Latin America.
Eduardo Teixeira obtained his $\mathrm{PhD}$ from the University of Texas at Austin in 2005 under the direction of Luis Caffarelli. He held a three-year Hill assistant professorship position at Rutgers University. In 2008 he returned to his native country, with the ambition to further contribute to the development of the Brazilian mathematical community. He became assistant professor and subsequently full professor of mathematics at the Universidade Federal do Ceará, the same university from which he had obtained his BS degree. He was awarded the Mathematical Congress of the Americas Prize in 2013 and was elected permanent fellow of the Brazilian Academy of Sciences in 2015. He has now returned to the United States as full professor of mathematics at the University of Central Florida. He tells the Notices: "Today, as a researcher, I like to think about mathematics outside the formality of the office. Some of my most creative ideas came to me during leisure times. In particular, the insight of treating degenerate equations as if it were a 'nonphysical' free boundary arrived to me when I was playing with my daughter, Amanda, at the beach."

The Ramanujan Prize is awarded annually to a young researcher from a developing country. The prize carries a cash award of US $\$ 15,000$, and the recipient is invited to deliver a lecture at ICTP.

\section{-From an ICTP-IMU announcement}

\section{Lim Awarded Smale Prize}

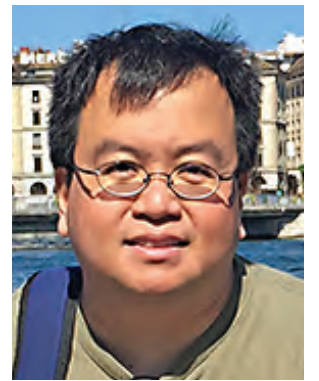

Lek-Heng Lim
LEK-HENG LIM of the University of Chicago has been awarded the third Stephen Smale Prize "for his outstanding contributions to the foundations of computational mathematics. His work seamlessly integrates scientific computing with complexity theory, statistical data analysis, and pure mathematics." He received his PhD in computational and mathematical engineering from Stanford University. He joined the faculty at Chicago in 2010, after serving as Charles Morrey Assistant Professor at the University of California, Berkeley. Lim tells the Notices: "I am a bit of a bibliophile. I particularly like Taipei, a city that still has many brick-and-mortar bookstores, some of which are open twenty-four hours. I am also a bit of a foodie. An appealing aspect of living in Chicago is that one can dine at a Michelin-starred restaurant for a little over $\$ 10$, although that pales in comparison to Hong Kong and Singapore, where a Michelin-starred meal can go for as low as \$2. In part to burn off these calories, I like biking and taking long walks with my wife along the magnificent trails next to Lake Michigan, Shing Mun River, or in the Singapore Botanic Gardens."

The Smale Prize is awarded every three years by the Society for the Foundations of Computational Mathematics. -Elaine Kehoe 


\section{Duminil-Copin Awarded Loève Prize}

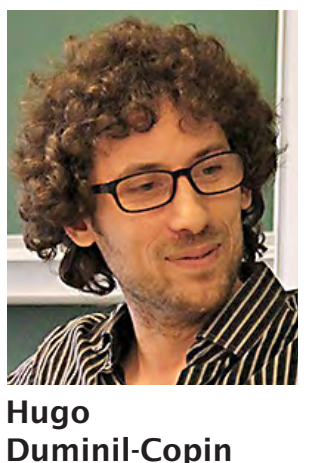

Hugo Duminil-Copin of the Institut des Hautes Etudes Scientifiques (IHES) Paris and the University of Geneva has been awarded the 2017 Loève International Prize in Probability. The prize citation reads in part: "He is best known for his early work on phase transitions in twodimensional lattice models: the Ising and Potts models, and properties of percolation and self-avoiding walks. Within these intensively studied fields, he and coauthors proved a wide range of long-standing hard conjectures for topics including the connective constant of the honeycomb lattice; critical points for random-cluster models; conformal invariance of the planar critical Ising and FK-Ising models; continuity of phase transitions and spontaneous magnetization in such models; and growth constants and critical fugacity of self-avoiding walks. Other major results involve sharp thresholds in more general settings, for bootstrap percolation as well as Bernoulli percolation and Ising models. Recently he and coauthors proved the long-standing Baxter's conjecture about continuity/discontinuity of phase transition for the planar Potts model." Duminil-Copin received his PhD in 2011 from the University of Geneva under the direction of Stanislav Smirnov. The Loève Prize is awarded every two years for outstanding contributions in probability by researchers under the age of forty-five. -David Aldous, University of California, Berkeley

\section{Pipher Named}

\section{Noether Lecturer}

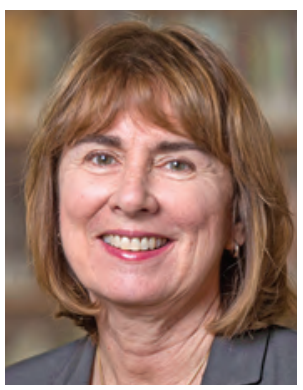

JILL PIPHER of Brown University has been named the 2018 Noether Lecturer by the Association for Women in Mathematics (AWM) and the AMS. She was honored "for her profound impact on mathematics, both through her work in the fields of harmonic analysis and partial differential equations and through her service to the profession." According to the prize citation, Pipher "is best known for her fundamental contriJill Pipher

butions to solutions and regularity of partial differential equations in minimally smooth domains"-for example, in her work with Verchota that "settled a long-standing conjecture on the solvability of the Dirichlet problem with $L^{2}$ boundary data on bounded Lipschitz domains." She has also done groundbreaking work in cryptography.
Pipher received her $\mathrm{PhD}$ from the University of California, Los Angeles and served on the faculty of the University of Chicago before joining Brown. She was the founding director of the Institute for Computational and Experimental Mathematics at Brown University. She was a member of the inaugural class of fellows of the AMS and served as president of AWM. She will give the Noether Lecture at the 2018 Joint Mathematics Meeting in San Diego, California. -From an AWM announcement

\section{Koberda Awarded 2017 Duszenko Prize}

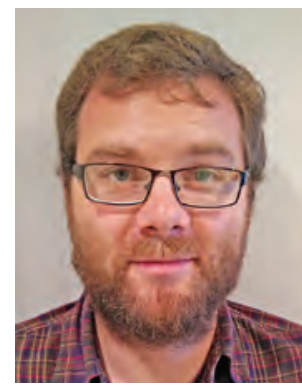

Thomas Koberda
THOMAS KOBERDA of the University of Virginia has been awarded the 2017 Kamil Duszenko Prize in Mathematics for his work on lowdimensional topology and dynamics, especially for a paper exploring connections between right-angled Artin groups and mapping class groups. He received his $\mathrm{PhD}$ from Harvard University in 2012 under the direction of Curt McMullen. From 2012 to 2015 he was both an NSF postdoc and assistant professor at Yale University; he joined the faculty at Virginia in 2015 . He received a Sloan Research Fellowship in 2017. Koberda tells the Notices: "I enjoy running, cooking, and wine. In my spare time, I love to read and to learn foreign languages."

The Duszenko Award is given by the Wrocław Mathematicians Foundation (WMF) for outstanding work or research that has significantly contributed to the deepening of knowledge and further progress in the field of mathematics. It was founded in honor of Kamil Duszenko, a young mathematician who died of acute lymphoblastic leukemia at the age of twenty-eight. It will be given at least every two years in the fields of mathematics and hematology.

-From a WMF announcement 


\section{NEWS}

\section{Dirac Medals Awarded}

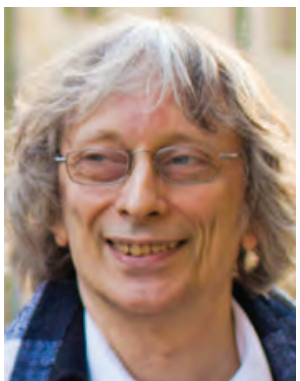

David Deutsch

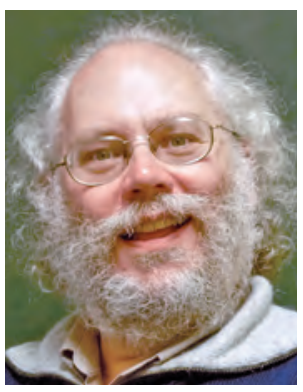

Peter W. Shor
The Dirac Medals for 2017 have been awarded by the International Centre for Theoretical Physics (ICTP) to CHARLES H. BENNETT of the IBM Watson Research Center, DAviD DEUTSCH of Oxford University, and PETER W. SHOR of the Massachusetts Institute of Technology "for their pioneering work in applying the fundamental concepts of quantum mechanics to solving basic problems in computation and communication and therefore bringing together the fields of quantum mechanics, computer science, and information." According to the prize citation, Bennett "proved that classical computation can be done without consumption of energy by inventing what is now known as reversible classical computation"; Deutsch "invented the notion of a quantum Turing machine, the concept of the quantum logic gate and quantum circuit, as well as the network model of computations"; and Shor "consolidated the field of quantum computation by designing the quantum algorithm for factoring large numbers." The medals are awarded to scientists who have made significant contributions to theoretical physics and carry a cash award of US\$5,000.

\section{-From an ICTP announcement}

\section{Mathematical Olympiad Results}

The team from South Korea finished first in the Fiftyeighth International Mathematical Olympiad (IMO) in Rio de Janeiro, Brazil. All six team members won gold medals. China finished second, followed by Vietnam, the United States, and Iran. Three of the US team members were awarded gold medals: ANKAN BHATTACHARYA, ANDREW Gu, and JAMES LIN. ZACHARY CHROMAN, VINCENT HUANG, and JUNYAO PENG received silver medals. Ankan also won a gold medal at the 2016 IMO and was the 2016 national Who Wants to Be a Mathematician champion. The 2018 Mathematical Olympiad will be held in Romania in July 2018.

-From MAA announcements

\section{MAA Awards Presented}

The Mathematical Association of America (MAA) awarded several writing and education prizes at its summer MathFest.

The Carl B. Allendoerfer Awards for excellent mathematical writing published in Mathematics Magazine were given to BRIAN CONREY and KENT MORRISON of the American Institute of Mathematics, along with JAMES GABBARD, University of Southern California; KATIE GRANT, University of California San Diego; and SHANG-CHI ANDREW LiU, University of California Los Angeles, all for the article "Intransitive Dice." VLADIMIR POZDNYAKOV, University of Connecticut, and Michael STEELE, University of Pennsylvania, were honored for their article "Buses, Bullies, and Bijections."

The Trevor Evans Award for excellent writing for an undergraduate audience published in Math Horizons was awarded to CORNELIA A. VAN COTT of the University of San Francisco for her article "A Pi Day of the Century Every Year."

The Paul R. Halmos-Lester R. Ford Awards for exceptional authors published in the American Mathematical Monthly were given to the following: HAROLD P. BOAS, Texas A\&M University, for his article "Mocposite Functions"; ADRIEN KASSEL, Ecole Normale Supérieure, and DAVID B. WILSON, Microsoft Research, for their article "The Looping Rate and Sandpile Density of Planar Graphs"; DEBORAH KenT, Drake University, and DAVID MURAKI, Simon Fraser University, for "A Geometric Solution of a Cubic by Omar Khayyam ... in which Colored Diagrams Are Used Instead of Letters for the Greater Ease of Learners"; and LAWRENCE ZALCMAN, Bar-Ilan University, for "A Tale of Three Theorems."

The George Pólya Awards for exceptional papers published in the College Mathematics Journal were awarded to VIKTOR BLÅSJÖ, Mathematical Institute of Utrecht University, for "How to Find the Logarithm of Any Number Using Nothing but a Piece of String" and TRAVIS KOWALSKI, South Dakota School of Mines and Technology, for "The Sine of a Single Degree."

The Merten M. Hasse Prize, for a noteworthy paper published by the MAA of which at least one of the authors is a younger mathematician, was awarded to LASSE REMPEGILLEN, University of Liverpool, and ZHAIMING SHEN, a graduate student at the University of Pennsylvania, for their paper "The Exponential Map Is Chaotic: An Invitation to Transcendental Dynamics" published in the American Mathematical Monthly.

The Daniel Solow Author's Award recognizes authors of undergraduate mathematics teaching materials. The 2017 inaugural recipient is TED SundSTROM of Grand Valley State University for Mathematical Reasoning: Writing and Proof.

The Henry L. Alder Awards honor beginning college or university faculty members whose teaching has been highly effective and successful in teaching undergraduate mathematics. The 2017 recipients are STEVEN KLEE of Seattle University, who is known for seamlessly incorporat- 
ing undergraduate research into his classroom curriculum and mentoring student researchers who go on to publish and present their work; and MARY BEISIEGEL of Oregon State University for her superb teaching, cultivating engaging classrooms, and her work building up professional development among her teaching peers.

The Mary P. Dolciani Award was presented to TATIANA SHubin of San Jose State University for her devotion to mathematical education at the K-12 student level, bringing Math Circles to new communities, particularly indigenous populations.

The Awards for Meritorious Service are presented for service at the national level or to a section of the MAA. The awardees are JAMES ALVAREZ, University of Texas at Arlington; SCOTT HOCHWALD, University of North Florida; HEIDI KECK, Western Colorado State University; JASON MOLITIERnO, Sacred Heart University; and GERARD A. Venema, Calvin College.

-From an MAA announcement

\section{*NSF Postdoctoral Research Fellowships Awarded}

The Mathematical Sciences Postdoctoral Research Fellowship Program of the Division of Mathematical Sciences (DMS) of the National Science Foundation (NSF) awards fellowships each year for postdoctoral research in pure mathematics, applied mathematics and operations research, and statistics. Following are the names of the fellowship recipients for 2017, together with their $\mathrm{PhD}$ institutions (in parentheses) and the institutions at which they will use their fellowships.

- KenNeTh AsCher (Brown University), Massachusetts Institute of Technology

- MAXIME Bergeron (University of British Columbia), University of Chicago

- Zarathustra BRAdy (Stanford University), Massachusetts Institute of Technology

- William Chan (California Institute of Technology), University of North Texas

- LAURA CLADEK (University of Wisconsin), University of British Columbia

- ERIN COMPAAN (University of Illinois UrbanaChampaign), Massachusetts Institute of Technology

- JeNNifer CRODELle (Rensselaer Polytechnic Institute), Courant Institute of Mathematical Sciences, New York University

- TheOdore Drivas (Johns Hopkins University), Princeton University

- MAX ENGELSTEIN (University of Chicago), Massachusetts Institute of Technology

\footnotetext{
"The most up-to-date listing of NSF funding opportunities from the Division of Mathematical Sciences can be found online at: www.nsf.gov/dms and for the Directorate of Education and Human Resources at www.nsf.gov/dir/index.jsp?org=ehr. To receive periodic updates, subscribe to the DMSNEWS listserv by following the directions at www.nsf.gov/mps/dms/about. jsp.
}

- Sylvester ERIKSSON-BIQUe (New York University), University of California, Los Angeles

- AleXANDRA FlOREA (Stanford University), University of Bristol

- Evan GAwLIK (Stanford University), University of California, San Diego

- Allen Gehret (Univerity of Illinois at Urbana-Champaign), University of California, Los Angeles

- NATE HARMan (Massachusetts Institute of Technology), University of Chicago

- SEAN Howe (University of Chicago), Stanford University

- Peter Jantsch (University of Tennessee), Texas A\&M University

- LIEN-Yung KAO (University of Notre Dame), University of Chicago

- Casey Kelleher (University of California, Irvine), Princeton University

- Alisa KNIZEL (Massachusetts Institute of Technology), Columbia University

- BRIAN LAWRENCE (Stanford University), Columbia University

- Oleg Lazarev (Stanford University), Columbia University

- DANIEL Le (University of Chicago), University of Toronto

- CAITLIN LeVERson (Duke University), Georgia Institute of Technology

- Christopher Lopez (University of California, Irvine), University of California, Santa Barbara

- LÁszLó M. LOVÁsz (Massachusetts Institute of Technology), University of California, Los Angeles

- Kyle J. LuH (Yale University), Harvard University

- Olya MANDElshtam (University of California, Berkeley), Brown University

- ANNA Medvedovsky (Brandeis University), Max Planck Institute for Mathematics

- Oliver Pechenik (University of Illinois at UrbanaChampaign), University of Michigan

- ThOMAs POLSTRa (University of Missouri), University of Utah

- ROHINi RAmadas (University of Michigan), Harvard University

- ERIC RAmos (University of Wisconsin-Madison), University of Michigan

- Donald Robertson (Ohio State University), University of Utah

- Elina Robeva (University of California, Berkeley), Massachusetts Institute of Technology

- CASEy Rodriguez (University of Chicago), Massachusetts Institute of Technology

- HenRi Roesch (Duke University), University of California, Irvine

- Nicholas SAlter (University of Chicago), Harvard University

- Kevin Schreve (University of Wisconsin-Milwaukee), University of Michigan-Ann Arbor

- AleXANDer Shapiro (University of California, Berkeley), University of Toronto

- MAtThew Stoffregen (University of California, Los Angeles), Massachusetts Institute of Technology 
- ARnav Tripathy (Stanford University), Harvard University

- Dootika VATs (University of Minnesota), University of Warwick

- IAN ZEMKE (University of California, Los Angeles), Princeton University

\section{-NSF announcement}

\section{Hidden Figures Honored with Book Award}

Author MARgot LeE SHETTERLy has received the 2017 Communication Award for Books from the National Academies of Sciences, Engineering, and Medicine for Hidden Figures: The American Dream and the Untold Story of the Black Women Mathematicians Who Helped Win the Space Race. The citation cites the book as "a hitherto little-known episode in the history of pioneering aerospace engineering and computing brought to light so engagingly that [it], along with the blockbuster movie it inspired, has had an unprecedented impact on the American public." The film made from the book was a finalist in the Film/ Radio/TV category. The communication awards, each of which includes a US\$20,000 prize, recognize excellence in reporting and communicating science, engineering, and medicine to the general public. A review of the film appeared in the June/July 2017 issue of the Notices. It can be retrieved at www . ams . org/publications/journals/ notices/201706/rnoti-p620.pd†.

-From a National Academies announcement

\section{Solomon Marcus (1925-2016)}

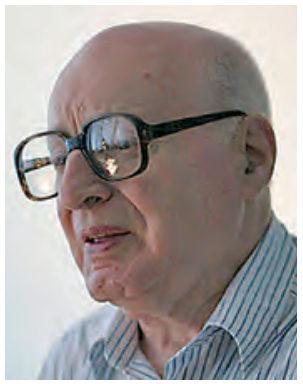

Solomon Marcus

SOLOMON MARCUS was a prolific and influential mathematician and interdisciplinary researcher who passionately worked until the end of his life. In the first ten years of his research career, he published almost 100 papers in mathematical analysis, set theory, measure and integration theory, and topology, including a joint paper with P. Erdôs, "Sur la décomposition de l'espace Euclidien en ensembles homogènes" (1957). He completed his studies in mathematics at the University of Bucharest, earning a PhD in 1956 (with the thesis Monotone Functions of Two Variables-in Romanian) under the supervision of M. Nicolescu and a State Doctorate in Sciences in 1968.

In the 1950s, the young Assistant Professor Solomon Marcus enchanted his students with the wealth of examples and problems that he brought to the analysis seminar.

From 1964 on he published many papers and books in theoretical computer science, linguistics, poetics and theory of literature, semiotics, cultural anthropology, biology, history and philosophy of science, and education. His book Grammars and Finite Automata (1964, in Romanian) is arguably the first monograph devoted to regular languages. He authored three pioneering books in mathematical linguistics and poetics: Introduction mathématique à la linguistique structurelle (1967), Algebraic Linguistics; Analytical Models (1967), and Mathematische Poetik (1973). His book Words and Languages Everywhere (2007) includes a collection of papers in language theories.

In a letter to the editor (Notices, 2009) on F. Dyson's "Birds and Frogs," he proposed a more refined typology of mathematicians inspired by F. Bacon's 1620 Novum Organum: ants, spiders, and bees. Ants (such as A. Zygmund) remain involved in one particular field, compensating by depth the lack of diversity; spiders (such as G. Cantor) propose a personal construction, with little reference to anybody else; and bees (such as P. Erdős) constantly move from problem to problem. He concluded with a few open problems, such as "Can we transfer these metaphors from individuals to historical periods?"

Solomon Marcus crossed many frontiers, geographical and transdisciplinary, and acquired not only erudition but also encyclopedic knowledge. As a public intellectual, he saved gifted scientists and scholars from marginalization. With equal ease, in his impeccable French, he magisterially debated the great French virologist Luc Montagnier a few years ago, on the stage of the Ateneul Român.

Marcus was a member of the Romanian Academy. He received many prizes, including the Royal Decoration of Nihil Sine Deo and the Star of Romania, Romania's highest civil order.

In his long career Marcus inspired, stimulated, encouraged, and advised many students, undergraduate and graduate, in Romania and abroad, to do mathematical research and, in the last decades, interdisciplinary studies. His sixteen PhD students form a small part of this group.

His 10-kilometer daily walk-his "constitutional"-may well have been one of the secrets of his great vitality and forward-looking exuberance.

He was never bored; he never felt the need of a proper vacation. In his last telephone conversation with Cristian Calude, when he was in the hospital, Solomon Marcus's strongest desire was to get back to work.

- Alexandra Bellow, Northwestern University

Cristian S. Calude, University of Auckland, New Zealand

\section{Photo Credits}

Photo of Eduardo Teixeira courtesy of Eduardo Teixiera. Photo of Lek-Heng Lim by Sou-Cheng Choi.

Photo of Hugo Duminil-Copin by Marie-Claude Vergne (IHES).

Photo of Jill Pipher (CBrown University.

Photo of Thomas Koberda by Angelo Mao.

Photo of David Deutsch by Lulie Tanett.

Photo of Peter W. Shor courtesy of Charles H. Bennett.

Photo of Solomon Marcus (c)Alexander Okhotin, used under the GFDL. 


\section{Inside the AMS}

\section{Class of Fellows of the AMS Selected}

A list of those who have been selected for the 2018 Class of Fellows of the AMS will appear on the AMS website beginning November 2, 2017. The list will be located at: www.ams.org/profession/new-fel1ows.

\section{New AMS Member Benefit}

On August 7, the AMS decided to implement a policy of free shipping for all members. This additional member benefit extends to all orders placed over the phone, online, at meetings, and more!

You can learn more about this and all benefits of membership at www . ams . org/membership.

\section{Deaths of AMS Members}

Tom M. ApOstoL, professor, California Institute of Technology, died on May 8, 2016. Born on August 20, 1923, he was a member of the Society for 71 years.

KARL F. BARTH, of Syracuse, New York, died on May 5, 2016. Born on September 25, 1938, he was a member of the Society for 53 years.

Heron S. Collins, professor, Louisiana State University, died on September 9, 2014. Born on November 17, 1922, he was a member of the Society for 62 years.

JiM DOUGLAS JR., professor, Purdue University , died on April 27, 2016. Born on August 8, 1927, he was a member of the Society for 65 years.

SOlOMON FEFERMAN, of Stanford, California, died on July 26, 2016. Born on December 13, 1928, he was a member of the Society for 65 years.

JOSEPH M. GANI, professor, Australian National University, died on April 12, 2016. Born on December 15, 1924, he was a member of the Society for 52 years.

SOLOMON W. GOLOMB, professor, University of Southern California, died on May 1, 2016. Born on May 31, 1932, he was a member of the Society for 61 years.

William H. GRAVES, of Chapel Hill, North Carolina, died on April 9, 2016. Born on July 4, 1940, he was a member of the Society for 49 years.
Rudolf Kalman, of Zurich, Switzerland, died on July 2, 2016. Born on May 19, 1930, he was a member of the Society for 57 years.

MARGARET J. KENNEY, of Quincy, Massachusetts, died on July 5,2016 . Born on June 7, 1935, she was a member of the Society for 53 years.

ANTE MIMICA, professor, University of Zagreb, died on June 9, 2016. Born on January 20, 1981, he was a member of the Society for 2 years.

JACQUES NeVEU, of Paris, France, died on May 17, 2016. Born on November 14, 1932, he was a member of the Society for 59 years.

SCOTT C. OTERMAT, of Fremont, Ohio, died on April 5, 2016. Born on September 30, 1946, he was a member of the Society for 42 years.

Thomas H. OTway, of Croton-on-Hudson, New York, died on April 5, 2016. Born on October 14, 1953, he was a member of the Society for 33 years.

MAXWELl O. READE, of Ann Arbor, Michigan, died on April 13, 2016. Born on April 11, 1916, he was a member of the Society for 76 years.

WALTER M. REID, of Eau Claire, Wisconsin, died on May 10, 2016. Born on June 4, 1943, he was a member of the Society for 40 years.

IVOR RoBINSON, of Dallas, Texas, died on May 27, 2016. Born on October 7, 1923, he was a member of the Society for 54 years.

JAMES T. ROGERS JR., of New Orleans, Louisiana, died on March 28, 2016. Born on July 26, 1942, he was a member of the Society for 50 years.

RONALD SHAw, of the United Kingdom, died on June 21, 2016. Born on September 26, 1960, he was a member of the Society for 25 years.

F. W. STAllmann, of Knoxville, Tennessee, died in September, 2014. Born on July 29, 1921, he was a member of the Society for 54 years.

PABlo Ulises SuAREZ, professor, Delaware State University, died on June 22, 2016. Born on October 4, 1980, he was a member of the Society for 4 years.

ANDREW H. VAN TUYL, of McLean, Virginia, died on April 11, 2016. Born on July 6, 1922, he was a member of the Society for 69 years.

CAROLYNE M. VAN VILET, professor, University of Miami, died on July 15, 2016. Born on December 27, 1929, she was a member of the Society for 7 years. 


\section{|| ||

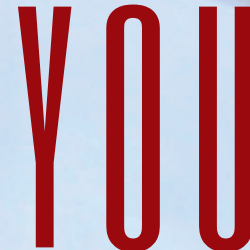

Visit the AMS Membership Booth to learn more about the benefits of membership!

The AMS is excited to announce a NEW benefit available to all individual members, FREE SHIPPING! In addition to receiving a discount on books purchased through the online bookstore and at meetings, members are also entitled to receive free shipping on their purchases. Join or renew your membership and receive a complimentary gift!
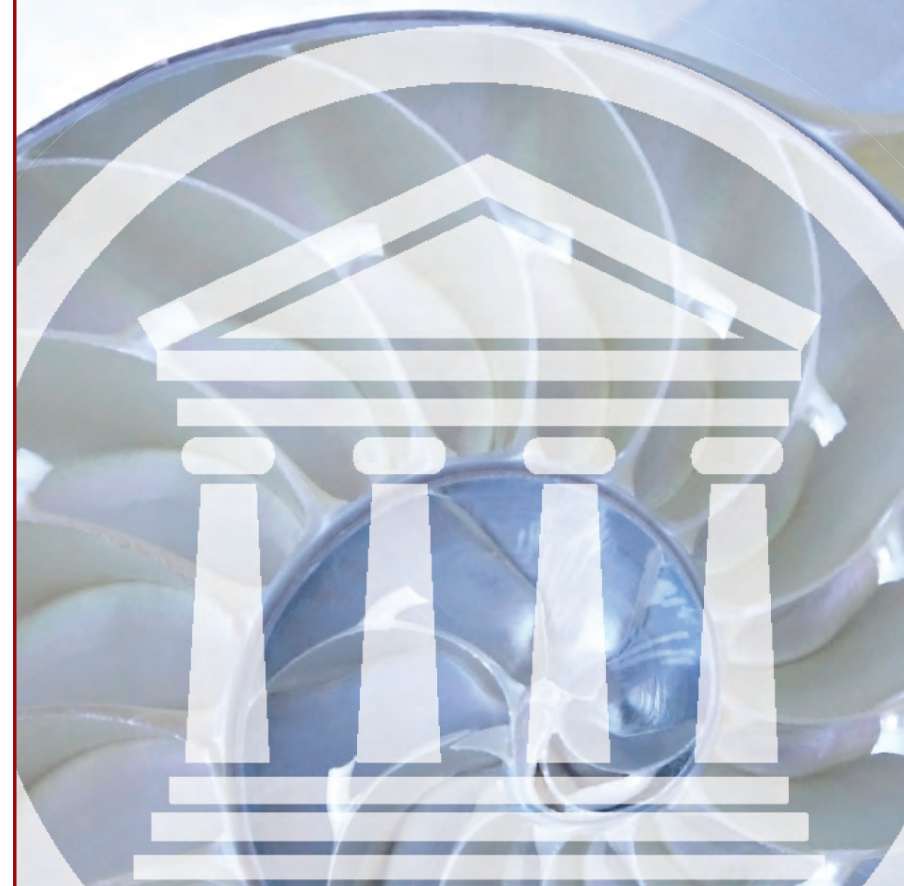

AMS Members, get your professional portrait taken at the Membership Booth!

The AMS Membership Department has arranged for a photographer to take your professional portrait and have it emailed to you in just a few minutes! You can upload this photo to your MathSciNet Author Profile page, use it on your University website, submit it as the professional photograph for your book publication, or use it as your profile picture in email and on social platforms.

Availability:

Thursday, January $11^{\text {th }} 2018$, 9:30 am$12: 30 \mathrm{pm}$ and Friday, January $12^{\text {th }} 2018$, 2:30 pm-5:30 pm.

Schedule your appointment at: amermathsoc.simplybook.me 


\section{New Publications Offered by the AMS}

To subscribe to email notification of new AMS publications, please go to www. ams. org/bookstore-emai 1.

\section{Algebra and Algebraic Geometry}

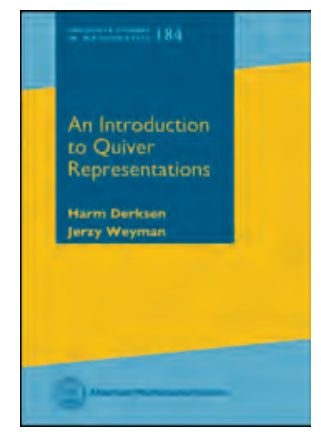

\section{An Introduction to Quiver Representations}

Harm Derksen, University of Michigan, Ann Arbor, and Jerzy Weyman, University of Connecticut, Storrs

An introduction to the representation theory of quivers and finite dimensional algebras, this book gives a thorough and modern treatment of the algebraic approach based on Auslander-Reiten theory as well as the approach based on geometric invariant theory. The material in the opening chapters is developed slowly with topics such as homological algebra, Morita equivalence, and Gabriel's theorem. Next, the book presents Auslander-Reiten theory, including almost split sequences and the Auslander-Reiten transform, and gives a proof of Kac's generalization of Gabriel's theorem. Once this basic material is established, the book goes on with developing the geometric invariant theory of quiver representations. It features the exposition of the saturation theorem for semi-invariants of quiver representations and its application to Littlewood-Richardson coefficients. In the final chapters, it exposes tilting modules, exceptional sequences and a connection to cluster categories.

This book is suitable for a graduate course in quiver representations and has numerous exercises and examples throughout the text. The book will also be of use to experts in such areas as representation theory, invariant theory and algebraic geometry, who want to learn about applications of quiver representations to their fields.

Contents: Introduction; Homological algebra of quiver representations; Finite dimensional algebras; Gabriel's theorem; Almost split sequences; Auslander-Reiten theory; Extended Dynkin quivers; Kac's theorem; Geometric invariant theory; Semi-invariants of quiver representations; Orthogonal categories and exceptional sequences; Cluster categories; Notation; Index; Bibliography.
Graduate Studies in Mathematics, Volume 184

December 2017, 344 pages, Hardcover, ISBN: 978-1-4704-2556-2, 2010 Mathematics Subject Classification: 16G20, 16G10, 16G70, 14L24, 13A50, AMS members US\$66.40, List US\$83, Order code GSM/184

\section{Analysis}

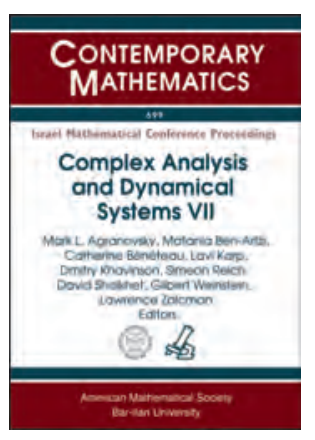

\section{Complex Analysis and Dynamical Systems VII}

Mark L. Agranovsky, Bar-Ilan University, Ramat-Gan, Israel, Matania Ben-Artzi, Hebrew University, Jerusalem, Israel, Catherine Bénéteau, University of South Florida, Tampa, Lavi Karp, ORT Braude College, Karmiel, Israel, Dmitry Khavinson, University of South Florida, Tampa, Simeon Reich, Technion, Haifa, Israel, David Shoikhet, Holon Institute of Technology, Israel, Gilbert Weinstein, Ariel University, Israel, and Lawrence Zalcman, Bar-Ilan University, Ramat-Gan, Israel, Editors

This volume contains the proceedings of the Seventh International Conference on Complex Analysis and Dynamical Systems, held from May 10-15, 2015, in Nahariya, Israel.

The papers in this volume range over a wide variety of topics in the interaction between various branches of mathematical analysis. Taken together, the articles collected here provide the reader with a panorama of activity in complex analysis, geometry, harmonic analysis, and partial differential equations, drawn by a number of leading figures in the field. They testify to the continued vitality of the interplay between classical and modern analysis. 
This book is co-published with Bar-Ilan University (Ramat-Gan, Israel).

Contents: A. Akinshin, G. Goldman, V. Golubyatnikov, and Y. Yomdin, Accuracy of reconstruction of spike-trains with two near-colliding nodes; L. Akinyemi, T. V. Savina, and A. A. Nepomnyashchy, Exact solutions to a Muskat problem with line distributions of sinks and sources; N. Albin, F. D. Sahneh, M. Goering, and P. Poggi-Corradini, Modulus of families of walks on graphs; A. Banyaga and P. Spaeth, Uniqueness of contact Hamiltonians of topological strictly contact isotopies; G. Barsegian, A triple principle (for curves, surfaces and complex functions) and the universal version of value distribution theory; R. Díaz Millán and A. Gibali, Characterization of orthogonal polynomials-A new proof of Bochner's theorem; V. Gichev, Decomposition of the Kostlan-Shub-Smale model for random polynomials; A. Golberg and R. Salimov, Differentiability of ring homeomorphisms with controlled $p$-module; V. V. Goryainov, Some inequalities for holomorphic self-maps of the unit disc with two fixed points; L. A. Harris, Interpolation and cubature at Geronimus nodes generated by different Geronimus polynomials; R. Hurri-Syrjänen and A. V. Vähäkangas, Characterizations to the fractional Sobolev inequality; L. A. Kalyakin, O. A. Sultanov, and N. Tarkhanov, Elliptic perturbations of dynamical systems with a proper node; M. Kapovich, Krull dimensions of rings of holomorphic functions; S. L. Krushkal, Complex geodesics and variational calculus for univalent functions; R. Kühnau, Analytic reflection across analytic Jordan curves; G. Liu and S. Ponnusamy, Compositions of polyharmonic mappings; D. S. Lubinsky, On Marcinkiewicz-Zygmund inequalities at Jacobi zeros and their Bessel function cousins; V. Matache, Nonminimal cyclic invariant subspaces of hyperbolic composition operators; C. Mills and M. Ru, An improved defect relation for holomorphic curves in projective varieties; S. Reich and A. J. Zaslavski, A porosity theorem for a class of nonexpansive set-valued mappings; H. Render, The Khavinson-Shapiro conjecture for domains with a boundary consisting of algebraic hypersurfaces; Y. B. Zelinskii, Shadow problem for the tangent bundle of straight lines on a sphere.

Contemporary Mathematics, Volume 699

November 2017, 312 pages, Softcover, ISBN: 978-1-4704-2961-4, LC 2017015043, 2010 Mathematics Subject Classification: 26-XX, 30-XX, 31-XX, 34-XX, 35-XX, 41-XX, 42-XX, 47-XX, 53-XX, 76-XX, 94XX, AMS members US\$88.80, List US\$111, Order code CONM/699

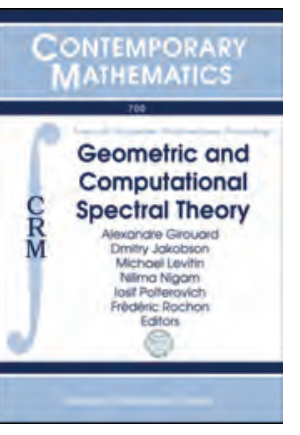

Geometric and Computational Spectral Theory

Alexandre Girouard, Université Laval, Québec, Canada, Dmitry Jakobson, McGill University, Montréal, Canada, Michael Levitin, University of Reading, United Kingdom, Nilima Nigam, Simon Frasier University, Burnaby, British Columbia, Canada, Iosif Polterovich, Université de Montréal, Canada, and Frédéric Rochon, Université du Québec à Montréal, Canada, Editors

The book is a collection of lecture notes and survey papers based on the mini-courses given by leading experts at the 2015 Séminaire de Mathématiques Supérieures on Geometric and Computational Spectral Theory, held from June 15-26, 2015, at the Centre de Recherches Mathématiques, Université de Montréal, Montréal, Quebec, Canada.

The volume covers a broad variety of topics in spectral theory, highlighting its connections to differential geometry, mathematical physics and numerical analysis, bringing together the theoretical and computational approaches to spectral theory, and emphasizing the interplay between the two.

This item will also be of interest to those working in applications.

This book is co-published with the Centre de Recherches Mathématiques.

Contents: B. Colbois, The spectrum of the Laplacian: A geometric approach; G. Berkolaiko, An elementary introduction to quantum graphs; D. Bucur and P. Freitas, A free boundary approach to the Faber-Krahn inequality; P. Bérard and B. Helffer, Some nodal properties of the quantum harmonic oscillator and other Schrödinger operators in $\mathbb{R}^{2}$; J. Bosch and C. Greif, Numerical solution of linear eigenvalue problems; G. Kanschat, Finite element methods for variational eigenvalue problems; A. Strohmaier, Computation of eigenvalues, spectral zeta functions and zeta-determinants on hyperbolic surfaces; D. Grieser, Scales, blow-up and quasimode constructions; C. Guillarmou, Scattering for the geodesic flow on surfaces with boundary.

Contemporary Mathematics, Volume 700

November 2017, 296 pages, Softcover, ISBN: 978-1-4704-26651, 2010 Mathematics Subject Classification: 58Jxx, 35Pxx, 65Nxx, AMS members US\$88.80, List US\$111, Order code CONM/700 


\section{Discrete Mathematics and Combinatorics}

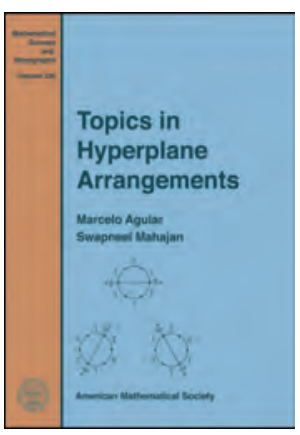

\section{Topics in Hyperplane Arrangements}

\section{Marcelo Aguiar, Cornell \\ University, Ithaca, NY, and Swapneel Mahajan, Indian Institute of Technology (IIT), Mumbai, India}

This monograph studies the interplay between various algebraic, geometric and combinatorial aspects of real hyperplane arrangements. It provides a careful, organized and unified treatment of several recent developments in the field, and brings forth many new ideas and results. It has two parts, each divided into eight chapters, and five appendices with background material.

Part I gives a detailed discussion on faces, flats, chambers, cones, gallery intervals, lunes and other geometric notions associated with arrangements. The Tits monoid plays a central role. Another important object is the category of lunes which generalizes the classical associative operad. Also discussed are the descent and lune identities, distance functions on chambers, and the combinatorics of the braid arrangement and related examples.

Part II studies the structure and representation theory of the Tits algebra of an arrangement. It gives a detailed analysis of idempotents and Peirce decompositions, and connects them to the classical theory of Eulerian idempotents. It introduces the space of Lie elements of an arrangement which generalizes the classical Lie operad. This space is the last nonzero power of the radical of the Tits algebra. It is also the socle of the left ideal of chambers and of the right ideal of Zie elements. Zie elements generalize the classical Lie idempotents. They include Dynkin elements associated to generic half-spaces which generalize the classical Dynkin idempotent. Another important object is the lune-incidence algebra which marks the beginning of noncommutative Möbius theory. These ideas are also brought upon the study of the Solomon descent algebra.

The monograph is written with clarity and in sufficient detail to make it accessible to graduate students. It can also serve as a useful reference to experts.

This item will also be of interest to those working in algebra and algebraic geometry.

Contents: Part I: Hyperplane arrangements; Cones; Lunes; Category of lunes; Reflection arrangements; Braid arrangement and related examples; Descent and lune equations; Distance functions and Varchenko matrix; Part II: Birkhoff algebra and Tits algebra; Lie and Zie elements; Eulerian idempotents; Diagonalizability and characteristic elements; Loewy series and Peirce decompositions; Dynkin idempotents; Incidence algebras; Invariant Birkhoff algebra and invariant Tits algebra; Appendices: Regular cell complexes; Posets; Incidence algebras of posets; Algebras and modules; Bands; Bibliography; Notation index; Subject index.

Mathematical Surveys and Monographs, Volume 226

November 2017, 608 pages, Hardcover, ISBN: 978-1-4704-3711-4, 2010 Mathematics Subject Classification: 05E10, 06A07, 06A11,
06C10, 16G10, 17B01, 20F55, 20M10, 20M25, 52C35; 05A18, 05A30, 05C25, 05E05, 05E18, 05E45, 18D50, 18G35, 20B30, AMS members US\$127.20, List US\$159, Order code SURV/226

\section{General Interest}

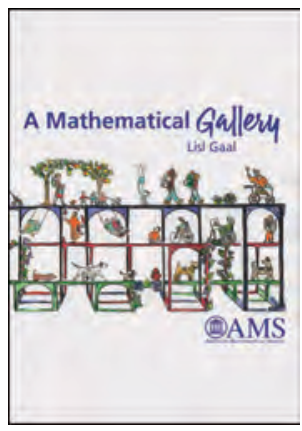

\section{A Mathematical Gallery}

\section{Lisl Gaal}

Embark on a playful mathematical tour, aided by Lisl Gaal's illustrations of familiar scenes and whimsical triggers for the imagination. Along the way, find fruit stands arranged using polynomial multiplication, checkerboard tablecloths sewed with patterns of primes in a two-dimensional number system, and deceptive cats revealing that simple counting is not always so simple.

Grasping the mathematics in this book requires only a basic background in algebra and geometry, so while the ideas can be understood and enjoyed at a variety of levels, it is recommended for ages 13-99. Touching on topics in current research, this is a book to read and revisit, gaining new insights each time.

Contents: Counting; The Pythagorean theorem; The volume of a pyramid; The area of a circle; Archimedes's proof for the volume of a sphere; Pascal's triangle; Gaussian integers; Permutations; The mathematics of probability and Markov processes; Desargue's theorem; Seven circle theorem; Calculus; Multiplying ordinals; A daughter's perspective.

November 2017, 64 pages, Softcover, ISBN: 978-1-4704-4159-3, LC 2017027805, AMS members US\$20, List US\$25, Order code MBK/111

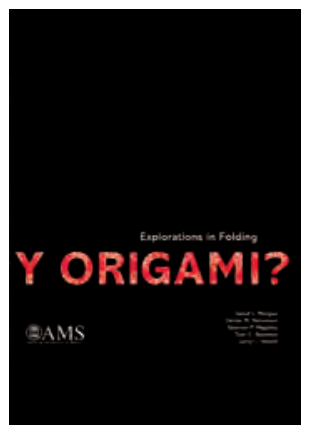

\section{Y Origami?} Explorations in Folding David C. Morgan, Denise M. Halverson, Spencer P. Magleby, Terri C. Bateman, and Larry L. Howell, all of Brigham Young University, Provo, UT

When origami met the worlds of design and engineering, both fields embraced the ancient art form, using its principles and practices to discover new problems and to generate inventive solutions.

This book demonstrates the potential of folding to improve the way things work, simplify how products are produced, and make possible new objects otherwise impossible. The solar collector, the felt stool, and the surgery tool have all been influenced in some way by folding paper. The example section is organized to show the folded figure next to the product prototype that was inspired by that work of origami. Included are models made from an array of materials over a range of sizes. This includes everything from a microscopic mechanism to huge solar panels designed to unfold in outer space. Most entries are at the prototype phase-physical 
hardware has been built to demonstrate the concept, but examples are not necessarily available commercially.

Y Origami? also includes brief learning activities related to paper folding, such as a discussion of Euler's formula, angular measurements, and developable surfaces, along with more advanced topics. Throughout the book many diagrams and photographs illustrate the advancing concepts and methods of origami as an art form and a problem-solving strategy.

Contents: Origami-based design: Deployable solar array; Ballistic barrier; Oriceps; Monolithic pointer; Nanoinjector; Tessel jet pack backpack; Circle/circle table; Plywood hinge bowl; O-rectractor; C-arm shroud; Backpackable solar array; Collapsible camp stove; Felt stool; Lens lift; Ruffled lamp; Morphing surface; Whole beauty bag; Oruga shelter; Bellows; Biopsy tool; Kaleidocycle bowls; Kinetic sculpture; Morphing antenna; Folded bundt pan; Learning activities: Euler's formula; Geometric patterns; Finding area; Finding volume; One straight cut design; Thick square twist; Oriceps; Nanoinjectors; Modularity; Angular measurements; Developable surfaces; Pop up; Hyperbolic paraboloid; Bellows; D-CORE catapult; Unwrapping sine curve; Advanced activities: Folding along a curve; Flat folding condition; Layering of folds; Kawaski's big-little-big angle theorem; Flat foldable vertex degree; Hull's consecutive sectors; Half plane theorems; New origami: Rigidly foldable tessellations; Mother and child; Exhibitions/Citations; Acknowledgements; Funding sources.

December 2017, 152 pages, Softcover, ISBN: 978-1-4704-3674-2, LC 2017002522, 2010 Mathematics Subject Classification: 00A06, 00A08, 00A09, 00A66, 97A20, 97A80, 97M10, 97M50, 97M80, AMS members US\$31.20, List US\$39, Order code MBK/104

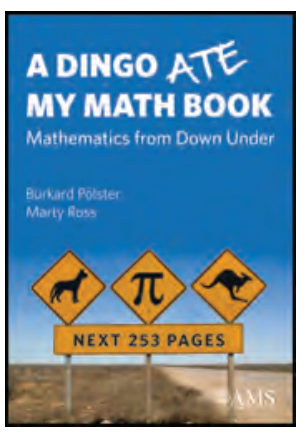

\section{A Dingo Ate My Math Book Mathematics from Down Under

\author{
Burkard Polster, Monash \\ University, Melbourne, and \\ Marty Ross, The University of \\ Queensland, Brisbane
}

A Dingo Ate My Math Book presents ingenious, unusual, and beautiful nuggets of mathematics with a distinctly Australian flavor. It focuses, for example, on Australians' love of sports and gambling, and on Melbourne's iconic, mathematically inspired architecture. Written in a playful and humorous style, the book offers mathematical entertainment as well as a glimpse of Australian culture for the mathematically curious of all ages.

This collection of engaging stories was extracted from the Maths Masters column that ran from 2007 to 2014 in Australia's Age newspaper. The maths masters in question are Burkard Polster and Marty Ross, two (immigrant) Aussie mathematicians, who each week would write about math in the news, providing a new look at old favorites, mathematical history, quirks of school mathematics-whatever took their fancy. All articles were written for a very general audience, with the intention of being as inviting as possible and assuming a minimum of mathematical background.

Contents: A day in Australia; Sports rules!; Aussie heroes; Melbourne, city of mathematics; A lotto gambling; Keeping the bastards honest; Canned life; Money, money, money; Family life;
Here, there and everywhere; TV snacks; The Australian math wars; The critics at work; Appendix.

October 2017, 272 pages, Softcover, ISBN: 978-1-4704-3521-9, LC 2017014890, 2010 Mathematics Subject Classification: 00A08, 00A09, 00A67, 97A80, AMS members US\$31.20, List US\$39, Order code $\mathrm{MBK} / 106$

\section{Geometry and Topology}

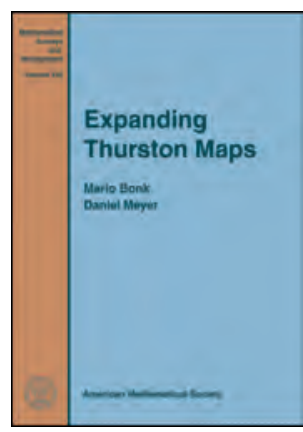

\section{Expanding Thurston Maps}

\author{
Mario Bonk, University of \\ California, Los Angeles, and \\ Daniel Meyer, University of \\ Liverpool
}

This monograph is devoted to the study of the dynamics of expanding Thurston maps under iteration. A Thurston map is a branched covering map on a two-dimensional topological sphere such that each critical point of the map has a finite orbit under iteration. A Thurston map is called expanding if, roughly speaking, preimages of a fine open cover of the underlying sphere under iterates of the map become finer and finer as the order of the iterate increases.

Every expanding Thurston map gives rise to a fractal space, called its visual sphere. Many dynamical properties of the map are encoded in the geometry of this visual sphere. For example, an expanding Thurston map is topologically conjugate to a rational map if and only if its visual sphere is quasisymmetrically equivalent to the Riemann sphere. This relation between dynamics and fractal geometry is the main focus for the investigations in this work.

Contents: Introduction; Thurston maps; Lattès maps; Quasiconformal and rough geometry; Cell decompositions; Expansion; Thurston maps with two or three postcritical points; Visual metrics; Symbolic dynamics; Tile graphs; Isotopies; Subdivisions; Quotients of Thurston maps; Combinatorially expanding Thurston maps; Invariant curves; The combinatorial expansion factor; The measure of maximal entropy; The geometry of the visual sphere; Rational Thurston maps and Lebesgue measure; A combinatorial characterization of Lattès maps; Outlook and open problems; Appendix A; Bibliography; Index.

Mathematical Surveys and Monographs, Volume 225 November 2017, 496 pages, Hardcover, ISBN: 978-0-8218-7554-4, LC 2017017476, 2010 Mathematics Subject Classification: 37-02, 37F10, 37F20, 30D05, 30L10, AMS members US\$135.20, List US\$169, Order code SURV/225 


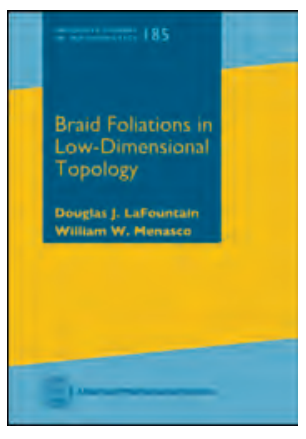

Braid Foliations in Low-Dimensional Topology

Douglas J. LaFountain, Western Illinois University, Macomb, and William W. Menasco, University at Buffalo, NY

This book is a self-contained introduction to braid foliation techniques, which is a theory developed to study knots, links and surfaces in general 3-manifolds and more specifically in contact 3-manifolds. With style and content accessible to beginning students interested in geometric topology, each chapter centers around a key theorem or theorems. The particular braid foliation techniques needed to prove these theorems are introduced in parallel, so that the reader has an immediate "take-home" for the techniques involved.

The reader will learn that braid foliations provide a flexible toolbox capable of proving classical results such as Markov's theorem for closed braids and the transverse Markov theorem for transverse links, as well as recent results such as the generalized Jones conjecture for closed braids and the Legendrian grid number conjecture for Legendrian links. Connections are also made between the Dehornoy ordering of the braid groups and braid foliations on surfaces.

All of this is accomplished with techniques for which only mild prerequisites are required, such as an introductory knowledge of knot theory and differential geometry. The visual flavor of the arguments contained in the book is supported by over 200 figures.

Contents: Links and closed braids; Braid foliations and Markov's theorem; Exchange moves and Jones' conjecture; Transverse links and Bennequin's inequality; The transverse Markov theorem and simplicity; Botany of braids and transverse knots; Flypes and transverse nonsimplicity; Arc presentations of links and braid foliations; Braid foliations and Legendrian links; Braid foliations and braid groups; Open book foliations; Braid foliations and convex surface theory; Bibliography; Index.

Graduate Studies in Mathematics, Volume 185

November 2017, 304 pages, Hardcover, ISBN: 978-1-4704-3660-5, LC 2017016585, 2010 Mathematics Subject Classification: 57M25, 57R17, 57M50, 57M27, 20F36, AMS members US\$66.40, List US\$83, Order code GSM/185

\section{Mathematical Physics}

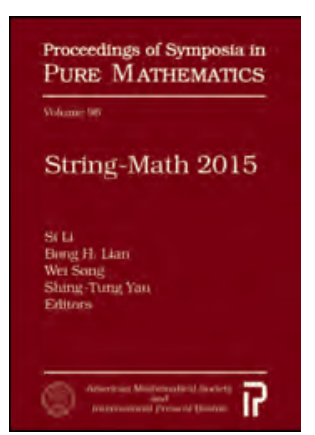

\section{String-Math 2015}

\author{
Si Li, Tsinghua University, \\ Beijing, China, Bong H. Lian, \\ Brandeis University, Waltham, \\ MA, Wei Song, Tsinghua \\ University, Beijing, China, and \\ Shing-Tung Yau, Harvard \\ University, Cambridge, MA, \\ Editors
}

This volume contains the proceedings of the conference String-Math 2015, which was held from December 31, 2015-January 4, 2016, at Tsinghua Sanya International Mathematics Forum in Sanya, China. Two of the main themes of this volume are frontier research on Calabi-Yau manifolds and mirror symmetry and the development of non-perturbative methods in supersymmetric gauge theories. The articles present state-of-the-art developments in these topics.

String theory is a broad subject, which has profound connections with broad branches of modern mathematics. In the last decades, the prosperous interaction built upon the joint efforts from both mathematicians and physicists has given rise to marvelous deep results in supersymmetric gauge theory, topological string, M-theory and duality on the physics side, as well as in algebraic geometry, differential geometry, algebraic topology, representation theory and number theory on the mathematics side.

This book is co-published with International Press of Boston.

Contents: K. Becker and M. Becker, Superstring compactifications to all orders in $\alpha^{\prime}$; F. Benini and A. Zaffaroni, Supersymmetric partition functions on Riemann surfaces; H.-L. Chang, J. Li, W.-P. Li, and C.-C. M. Liu, On the mathematics and physics of Mixed Spin P-fields; C.-H. Cho, Homological mirror functors via Maurer-Cartan formalism; C. F. Doran, A. Harder, and A. Thompson, Mirror symmetry, Tyurin degenerations and fibrations on Calabi-Yau manifolds; M. Han, SL(2, C) Chern-Simons theory and four-dimensional quantum geometry; Y.-P. Lee, H.-W. Lin, and C.-L. Wang, Quantum cohomology under birational maps and transitions; G. W. Moore, A. B. Royston, and D. Van den Bleeken, $L^{2}$-kernels of Dirac-type operators on monopole moduli spaces; N. Nekrasov, $\mathcal{B P S} / C \mathcal{F} \mathcal{T}$ correspondence: Instantons at crossroads and gauge origami; $\boldsymbol{X}$. Wang and $\mathbf{Y}$. Zhang, Balanced embedding of degenerating Abelian varieties; N. Yui, The modularity/automorphy of Calabi-Yau varieties of CM type.

Proceedings of Symposia in Pure Mathematics, Volume 96 November 2017, 295 pages, Hardcover, ISBN:978-1-4704-29515, LC 2017021722, 2010 Mathematics Subject Classification: 14N35, 51P05, 53D37, 53D45, 53Z05, 81T20, 81T30, 81T60, AMS members US\$100.80, List US\$126, Order code PSPUM/96 


\section{New AMS-Distributed Publications}

\section{General Interest}

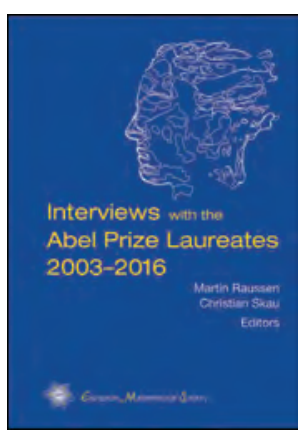

\section{Interviews with the Abel Prize Laureates 2003-2016}

Martin Raussen, Aalborg University, Denmark, and Christian Skau, Norwegian University of Science and Technology, Trondheim, Editors

The Abel Prize has been awarded annually to mathematicians in recognition of pioneering scientific achievements. Since the first award in 2003, Martin Raussen and Christian Skau have had the opportunity to conduct extensive interviews with the laureates. The interviews from 2003-2016 have been collected in this edition. They highlight the mathematical achievements of the laureates, place them in a historical perspective, and then try to unravel the way in which the world's most famous mathematicians conceive and judge their results, how they collaborate with peers and students, and how they perceive the importance of mathematics to society.

A publication of the European Mathematical Society.

September 2017, 301 pages, Softcover, ISBN: 978-3-03719-177-4, 2010 Mathematics Subject Classification: 01A70, 01A60, 01A61, 01A80, 00A35, AMS members US\$25.60, List US\$32, Order code EMSAPL

\section{Geometry and Topology}

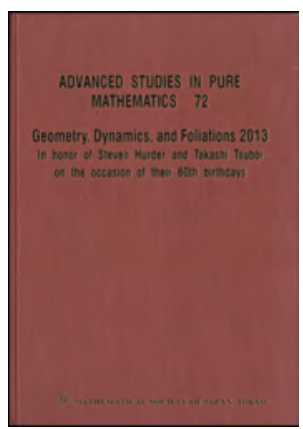

\section{Geometry, Dynamics, and Foliations 2013}

In Honor of Steven Hurder and Takashi Tsuboi on the Occasion of Their 60th Birthdays

Taro Asuke, University of Tokyo, Japan, Shigenori Matsumoto, Nihon University, Tokyo, Japan, and Yoshihiko Mitsumatsu, Chuo University, Tokyo, Japan, Editors
This volume aims to provide a good overview of present studies as well as some perspective for further studies. It includes B. Deroin's survey on Brownian motions on foliated complex surfaces, which contains many essential results and ideas.

Other articles cover a wide range of topics related to foliations and groups of diffeomorphisms old and new; some articles discuss characteristic classes such as Godbillon-Vey class or homotopy theory of foliations while others deal with topological and differential geometrical aspects, particularly in the holomorphic setting. Several articles show the relationship of these topics to geometric group theory.

This volume is highly recommended to researchers and graduate students interested in the theories of foliations and related topics such as diffeomorphism groups and geometric group theory.

This item will also be of interest to those working in analysis.

Published for the Mathematical Society of Japan by Kinokuniya, Tokyo, and distributed worldwide, except in Japan, by the AMS.

Advanced Studies in Pure Mathematics, Volume 72, August 2017, 451 pages, Hardcover, ISBN: 978-4-86497-040-2, 2010 Mathematics Subject Classification: 57R30; 37F75, 57R32, 58D05, 58H05, AMS members US\$77.60, List US\$97, Order code ASPM/72

\section{Math Education}

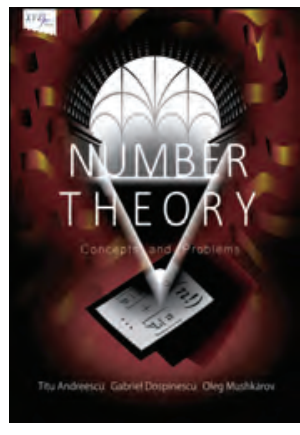

\section{Number Theory: Concepts and Problems}

Titu Andreescu, University of Texas at Dallas, Gabriel Dospinescu, Ecole Normale Superieure, Lyon, France, and Oleg Mushkarov, Bulgarian Academy of Science, Sofia, Bulgaria

This book is a powerful collection of competition problems with number theoretical flavor. They are generally grouped according to common aspects, related to topics such as divisibility, GCD and LCM, decomposition of polynomials, congruences and $p$-adic valuations.

Each chapter focuses on a fundamental concept or result, reinforced by each of the subsections, with scores of challenging problems.

A publication of XYZ Press. Distributed in North America by the American Mathematical Society.

XYZ Series, Volume 27 July 2017, 686 pages, Hardcover, ISBN: 978 0-9885622-0-2, 2010 Mathematics Subject Classification: 00A05, 00A07, 97U40, 97D50, AMS members US\$63.96, List US\$79.95, Order code XYZ/27 


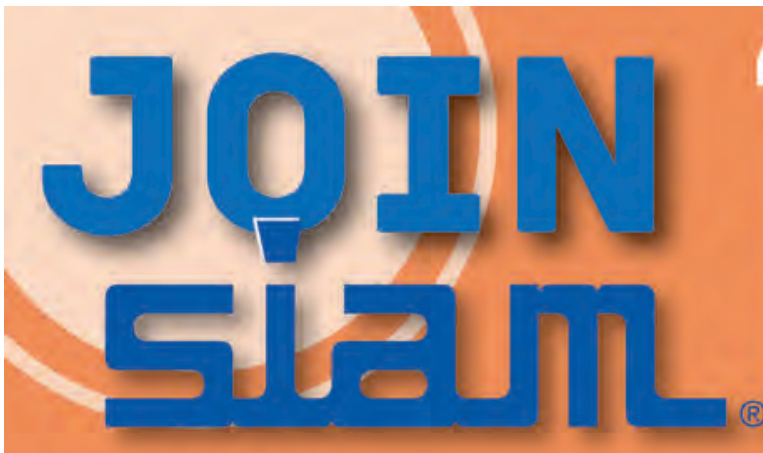

More than 14,500 mathematicians, computer scientists, engineers, physicists, and other scientists enjoy the many benefits of belonging to the Society for Industrial and Applied Mathematics. SIAM members are researchers, educators, practitioners, and students from more than 100 countries working in industry, laboratories, government, and academia.

MEMBERS OF STAM HAVE ACGESS TO:

\section{- SIAM News and SIAM Review}

- Discounts on books, journals, and conferences

- SIAM Activity Groups

- Participation in SIAM elections, leadership opportunities, and the SIAM Fellows program

- Networking opportunities

- Career Resources

- Resources and support for student activities

YOU ARE INVITED TO JOIN SIAM AND BE A PART OF OUR INTERNATIONAL AND INTERDISCIPLINARY COMMUNITY. ENTER PROMO CODE MBAM18 AT CHECKOUT WHEN YOU JOIN BY 12/31/17 AND GET 25\% OFF YOUR MEMBERSHIP!

JOIN TODAY: WWW.SIAM.ORG/JOINSIAM

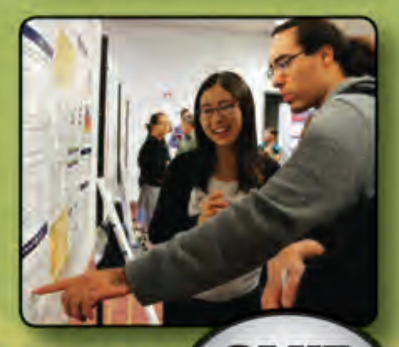

SAVE $25 \%$

SOCIETY for INDUSTRIAL and APPLIED MATHEMATICS 3600 Market Street, 6th Floor, Philadelphia, PA 19104-2688 USA Phone: +1-215-382-9800-Fax: +1-215-386-7999·membership@siam.org.www.siam.org

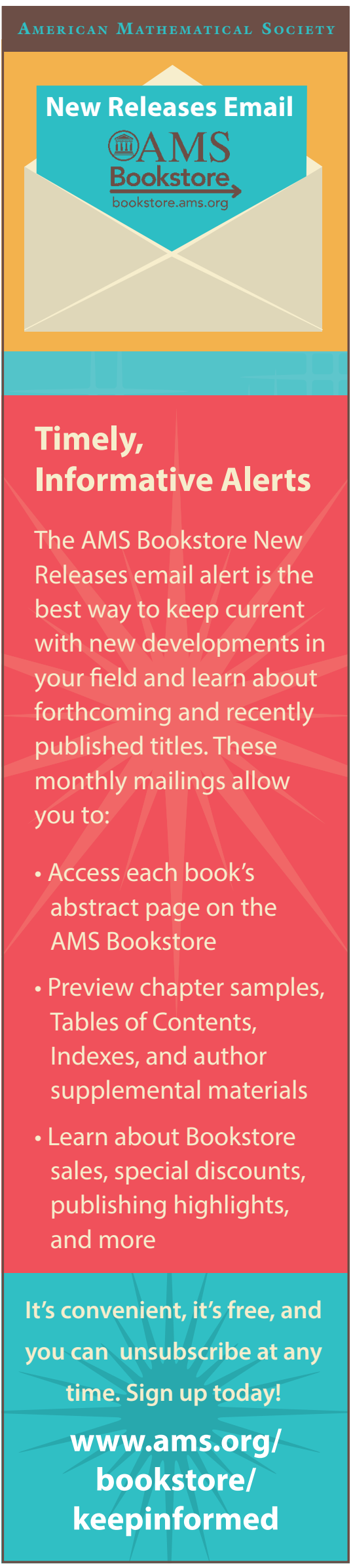




\begin{abstract}
As of the January 2018 issue of Notices, Mathematics Calendar will be an online-only product.

The AMS invites you to submit announcements of worldwide meetings and conferences of interest to the mathematical public, including ad hoc, local, or regional meetings, and meetings and symposia devoted to specialized topics, as well as announcements of regularly scheduled meetings of national or international mathematical organizations to the interactive calendar at www.ams.org/cgi-bin/mathca1/ mathca7-submit.p7.

Mathematics Calendar is available at: www.ams.org/ meetings/calendar/mathcal

Any questions about or difficulties with submissions may be directed to mathca1@ams.org.
\end{abstract}

\section{October 2017}

19 - 19 Next ITS-CUNY Symposium on Nonlinear Problems on Geometry

Location: The Graduate Center, The City University of New York, 365 Fifth Ave., New York, New York 10016.

URL: www.math.csi.cuny.edu/ huang/geo.pdf

23 - 27 Fixed Point Theory in Probabilistic and Fuzzy Structures: Under Global Initiative of Academic Networks (GIAN), MHRD, Government of India

Location: Guru Ghasidas Vishwavidyalaya (A Central University), Bilaspur (Chhattisgarh State), 495 9, India.

URL: www.gian. i itkgp.ac. in/ccourses/approvecourses 3

27 - 27 The IV Workshop on Computational Data Analysis and Numerical Methods (WCDANM)

Location: Beja, Portugal.

URL: www.wcdanm-beja17.uevora.pt

28 - 29 National Conference on "Discrete Mathematics, Theoretical Computer Science, Computer Engineering, and Applications"

Location: Department of Mathematics, T. D. P. G. College, Jaunpur - 222002 (U.P.) India.

URL: rsmams.org/newsdetai1s.php?id=23

28 - 30 Twenty-First International Conference of International Academy of Physical Sciences (CONIAPS XXI)

Location: Guru Jambheshwar University of Science and Technology Hisar-125001 (Haryana), India.

URL: gjuonline.ac.in/coniapsxxi

30 - 31 National Conference on Ramanujan: A Goddess Gifted Mathematician

Location: Department of Mathematics, T. D. P. G. College, Jaunpur - 222002 (U.P.) India.

URL: rsmams.org/newsdetai1s.php?id=24

30 - November 3 International Autumn School on Computational Number Theory

Location: Izmir Institute of Technology, Izmir, Turkey.

URL: ascnt2017.wordpress.com
November 2017

2 - 4 International Conference on Special Functions and Applications (ICSFA-2017) (XVIth Annual Conference of Society for Special Functions and Their Applications)

Location: Department of Mathematics, College of Engineering and Technology, Bikaner (An Autonomous Institution of Government of Rajasthan) Karni Industrial Area, Pugal Road, Bikaner-334004, Rajasthan, India.

URL: www.ssfaindia.webs.com/conf.htm

3 - 52017 Field of Dreams Conference

Location: Renaissance St. Louis Airport Hotel $\square$ St. Louis, Missouri

URL: $\quad$ www.matha17iance.org/2017-field-of-dreamsconference

10 - 11 Third International Conference on Pure and Applied Mathematics

Location: Department of Mathematics, University of Sargodha, Sargodha, Pakistan.

URL: icpam.uos.edu.pk

December 2017

6 - 9 Riemannian Geometry Past, Present and Future: An homage to Marcel Berger

Location: IHES, Bures-sur-Yvette, France.

URL: https://indico.math.cnrs.fr/event/2432

7 - 10 T.I.M.E - Technology and Innovation in Math Education

Location: Rajagiri School of Engineering and Technology, Cochin, Kerala, India.

URL: www.time2017. in

9 - 11 International Conference on Algebra, Discrete Mathematics, and Applications (ICADMA 2017)

Location: Department of Mathematics, Dr. Babasaheb Ambedkar Marathwada University, Aurangabad 431004 India.

URL:

18 - 21 Tenth HiPC Student Research Symposium (SRS)

Location: Jaipur, India.

URL: www.hipc.org

22 - 24 International Conference on Machine Intelligence and Signal Processing (MISP 2017)

Location: Indian Institute of Technology Indore, India.

URL: misp.iiti.ac.in

28 - January 4, 2018 Theory and Application of Wavelets and Framelets

Location: India.

URL: iiti.ac.in/people/ nirajshuk1a/Gian.htm1 


\section{AMS Centennial Research Fellowship Program}

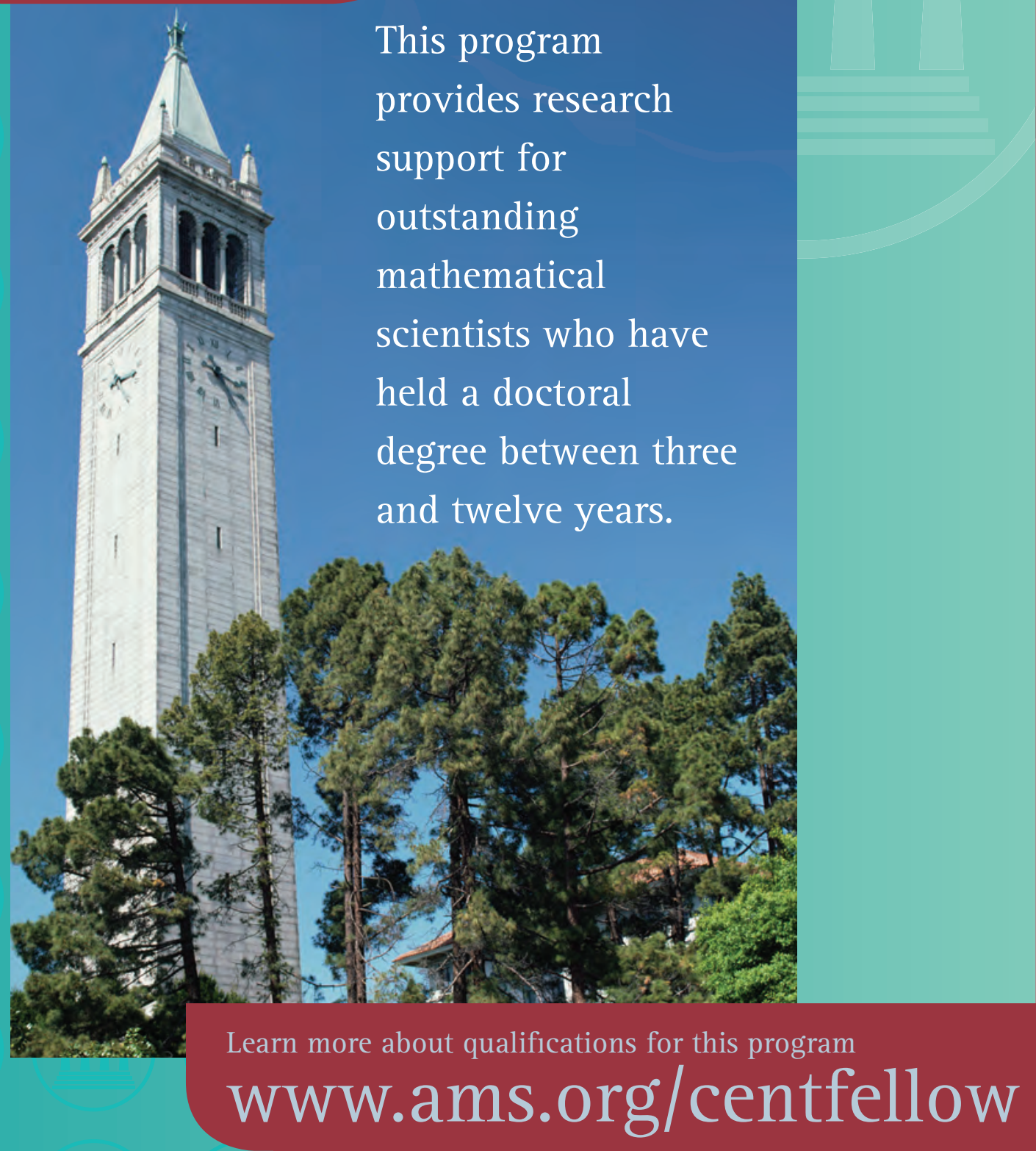

Application deadline: December 1, 2018

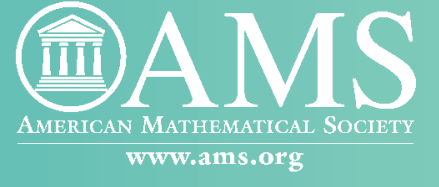




\section{Classified Advertisements}

\section{Positions available, items for sale, services available, and more}

\section{CALIFORNIA}

\section{Arthur J. Krener Assistant Professor Positions in Mathematics}

The Department of Mathematics at the University of California, Davis is soliciting applications for one or more Arthur J. Krener Assistant Professor positions starting July 1, 2018.

The Department seeks applicants with excellent research potential in areas of faculty interest and effective teaching skills. Applicants are required to have completed their $\mathrm{PhD}$ by the time of their appointment, but no earlier than July 1,2014 . The annual salary is $\$ 64,000$. The teaching load is 3 to 4 quarter-long courses. Krener appointments are renewable for a total of up to three years, upon demonstration of satisfactory performance in research and teaching.

Additional information about the Department may be found at www.math. ucdavis.edu/.

Applications will be accepted until the position is filled. To guarantee full consideration, the application should be received by November 30, 2017. To apply: submit the AMS Cover Sheet and supporting documentation electronically through www.mathjobs.org/.

The University of California, Davis, is an affirmative action/equal opportunity employer with a strong institutional commitment to the achievement of diversity among its faculty and staff.

00032

\section{Faculty Position in Mathematics University of California, Davis}

The Department of Mathematics at the University of California, Davis invites applications for an Assistant Professor (tenure-track) faculty position starting July 1, 2018.

Minimum qualifications for the position include a PhD degree or its equivalent in the Mathematical Sciences or a related field and demonstrated potential for performance in teaching and research. Duties include mathematical research, undergraduate and graduate teaching, and departmental, university and professional service.

Additional information about the Department may be found at www.math. ucdavis .edu/

Applications will be accepted until the position is filled. For full consideration, completed applications should be received by November 30, 2017. To apply: submit the AMS Cover Sheet and supporting documentation electronically through www.mathjobs.org/

The University of California, Davis, is an affirmative action/equal opportunity employer with a strong institutional commitment to the achievement of diversity among its faculty and staff.

00031

\section{Mathematical Sciences Research Institute (MSRI) Berkeley, CA}

\section{Director of Advancement \& External Relations}

Oppenheim Associates is assisting MSRI in the search for a new Director for Advancement \& External Relations. The

Suggested uses for classified advertising are positions available, books or lecture notes for sale, books being sought, exchange or rental of houses, and typing services. The publisher reserves the right to reject any advertising not in keeping with the publication's standards. Acceptance shall not be construed as approval of the accuracy or the legality of any advertising.

The 2017 rate is $\$ 3.50$ per word with a minimum two-line headline. No discounts for multiple ads or the same ad in consecutive issues. For an additional $\$ 10$ charge, announcements can be placed anonymously. Correspondence will be forwarded.

Advertisements in the "Positions Available" classified section will be set with a minimum one-line headline, consisting of the institution name above body copy, unless additional headline copy is specified by the advertiser. Headlines will be centered in boldface at no extra charge. Ads will appear in the language in which they are submitted.

There are no member discounts for classified ads. Dictation over the telephone will not be accepted for classified ads.

Upcoming deadlines for classified advertising are as follows: December 2017-September 28, 2017; January 2018-October 31, 2017; February 2018November 23, 2017; March 2018-January 2, 2018; April 2018-January 30, 2018; May 2018-March 2, 2018.

US laws prohibit discrimination in employment on the basis of color, age, sex, race, religion, or national origin. "Positions Available" advertisements from institutions outside the US cannot be published unless they are accompanied by a statement that the institution does not discriminate on these grounds whether or not it is subject to US laws. Details and specific wording may be found on page 1373 (vol. 44).

Situations wanted advertisements from involuntarily unemployed mathematicians are accepted under certain conditions for free publication. Call toll-free 800-321-4AMS (321-4267) in the US and Canada or 401-455-4084 worldwide for further information.

Submission: Promotions Department, AMS, P.O. Box 6248, Providence, Rhode Island 02904; or via fax: 401-331-3842; or send email to c7assads@ams .org. AMS location for express delivery packages is 201 Charles Street, Providence, Rhode Island 02904. Advertisers will be billed upon publication. 
organization seeks a proven fundraising professional to shape significant endowment campaigns, drive annual contributed revenue, and engage new donors from various fields of endeavor who will support fresh research into mathematics. For more information please review the complete Position Description at: www.moppenheim.com/wp-content/uploads/ MSRI-Director-for-Advancementposition-description-Final.pdf.

MSRI is the world's preeminent center for collaborative research in mathematics, and advances research into the key unsolved mathematical problems that underlie core mathematics and applications in the physical sciences, economics, engineering, computing, communications, statistical analysis and the global financial system. The prime objective for this position is to develop strategies and campaigns that:

- Develop a 5 year campaign to add $\$ 27 \mathrm{~m}$ to the current MSRI endowment of $\$ 23 \mathrm{~m}$, and then a second campaign to add another $\$ 50 \mathrm{~m}$ to the endowment by 2030 for a total endowment of $\$ 100$ million.

- Deliver Annual Fund Contributions of $\$ 4.5 \mathrm{~m}$ by 2020 (up from about $\$ 3.5 \mathrm{~m}$ in 2017).

- Diversifies and Broadens Donor Support for MSRI through use of conventional and social media marketing that communicates MSRI's relevance to donors of different sectors (tech, finance, economics, medicine, etc).

Founded in 1982 and located in Berkeley, MSRI has a 2017 budget of $\$ 9.9$ million, a staff of 21 , and a Board of 33 . The Advancement Team includes the Executive Director, an Associate Director, admin support, and subcontractors including but not limited to web development, videography, and design of communications materials.

For additional information or to apply, please contact Mark Oppenheim or Patrick Salazar at info@moppenheim.com .

More information can be found on the MSRI website and on Numberphile, an MSRI-supported YouTube channel with short and entertaining videos on mathematics.

00039

\section{University of California, Los Angeles Department of Mathematics Faculty Positions 2018-19}

Tenured/Tenure-Track positions 201819 (subject to administrative approval)

The Department of Mathematics at the University of California, Los Angeles, invites applications for tenure-track or tenured faculty positions starting July 1 , 2018. Outstanding candidates in all areas of mathematics may be considered.

Applicants must possess a PhD and should have outstanding accomplishments in both research and teaching. Duties include mathematical research, undergraduate and graduate teaching, and departmental and university service. Level of appointment will be based on qualifications, with appropriate salary per UC pay scales.

As a campus with a diverse student body, we encourage applications from women, minorities, and individuals with a history of mentoring under-represented minorities in the sciences. The Department of Mathematics welcomes candidates whose experience in teaching, research, or community service has prepared them to contribute to our commitment to diversity and excellence.

The University of California is an Equal Opportunity/Affirmative Action Employer. All qualified applicants will receive consideration for employment without regard to race, color, religion, sex, sexual orientation, gender identity, national origin, disability, age or protected veteran status. For the complete University of California nondiscrimination and affirmative action policy see: UC Nondiscrimination and Affirmative Action Policy (policy. ucop . edu/doc/4000376/NondiscrimAffi rmAct).

The University of California asks that applicants complete the Equal Opportunity Employer survey for Letters and Science at the following URL: cis.ucla. edu/facultysurvey/. Under Federal Law, the University of California may employ only individuals who are legally authorized to work in the United States as established by providing documents specified in the Immigration Reform and Control Act of 1986.

Applications and supporting documentation for all positions must be submitted online via www.mathjobs.org.

Applications will be accepted until the position is filled. To guarantee full consideration, the application should be received by November 15, 2017.

Temporary Faculty Positions 2018-19

The Department of Mathematics at the University of California, Los Angeles, invites applications for temporary and visiting appointments in the categories 1-4 below. Depending on the level, candidates must give evidence of potential or demonstrated distinction in scholarship and teaching. Applicants must possess a $\mathrm{PhD}$ and should have outstanding accomplishments in both research and teaching. Postdoctoral Positions:

(1) E.R. Hedrick Assistant Professorships: Appointments are for three years. The teaching load is four one-quarter courses per year.

(2) Computational and Applied Mathematics (CAM) Assistant Professorships: Appointments are for three years. The teaching load is normally reduced by research funding to two one-quarter courses per year.

(3) Program in Computing (PIC) Assistant Adjunct Professorships: Applicants for these positions must show very strong promise in teaching and research in an area related to computing. The teaching load is four one-quarter programming courses each year and one additional course every two years. Initial appointments are for one year and possibly longer, up to a maximum service of four years.

(4) Assistant Adjunct Professorships and Research Postdocs: Appointments are normally for one year, with the possibility of renewal. Strong research and teaching background required. The teaching load for is six one-quarter courses per year.

Appointments will be effective July 1, 2018 or later. Applications will be accepted until all positions are filled. For fullest consideration, all application materials should be submitted on or before November 15, 2017.

Applications and supporting documentation must be submitted online via www . mathjobs.org.

All letters of evaluation are subject to UCLA campus policies on confidentiality. Refer potential reviewers to the UCLA statement of confidentiality at www. apo.ucla.edu/policies/the-ca11/ summary-ot-procedures/summary10-statement-of-confidentiality.

\section{Lecturer Positions in Mathematics}

The UCLA Department of Mathematics receives on an ongoing basis applications for quarter positions (Fall/Winter/Spring or for Summer Session) for Lecturers to teach undergraduate Mathematics, Financial Actuarial Mathematics, or Math Education courses. Positions are very limited and temporary. Responsibilities include lecturing, conducting office hours, writing and grading exams and supervising teaching assistants. Previous teaching experience at the college level or extensive actuary experience is required and a $\mathrm{PhD}$ is preferred. Applications will be accepted until all positions are filled.

Applications and supporting documentation must be submitted online via www. mathjobs.org.

00023

\section{MASSACHUSETTS}

\section{Massachusetts Institute of Technology Cambridge, MA}

The Mathematics Department at MIT is seeking to fill positions in Pure and Applied Mathematics at the level of Assistant Professor or higher beginning July 2018 (for the 2018-2019 academic year, or as soon thereafter as possible). Appointments are based primarily on exceptional research qualifications. Appointees will be required to fulfill teaching duties and pursue their own research program. $\mathrm{PhD}$ in Mathematics or related field required by employment start date.

For more information and to apply, please visit www.mathjobs.org. To receive full consideration, submit applica- 
tions by December 1, 2017. MIT is an Equal Opportunity, Affirmative Action Employer.

\section{Massachusetts Institute of Technology} Cambridge, MA

The Mathematics Department at MIT is seeking to fill positions in Pure and Applied Mathematics, and Statistics at the level of Instructor beginning July 2018 (for the 2018-2019 academic year). Appointments are based primarily on exceptional research qualifications. Appointees will be expected to fulfill teaching duties and pursue their own research program. $\mathrm{PhD}$ in Mathematics or related field required by employment start date.

For more information and to apply, please visit www. mathjobs.org. To receive full consideration, submit applications by December 1,2017 . MIT is an Equal Opportunity, Affirmative Action Employer.

\section{Northeastern University Department of Mathematics Assistant/Associate Professor Tenure-Track Position}

The Department of Mathematics at Northeastern University invites applications for a tenure-track position at the Assistant/ Associate Professor level in Mathematics to start as early as Fall of 2018.

Appointments will be based on exceptional research contributions in Mathematics combined with a strong commitment and demonstrated success in teaching. Outstanding candidates in all areas of Pure and Applied Mathematics are encouraged to apply and will be seriously considered. Some of the Department goals and strengths are the following:

In Core Mathematics, the Department has developed a strong research program in Geometry, Physics, and Representation Theory, and seeks to build on this center of excellence by hiring in these and in synergetic areas, such as Topology, Combinatorics, Symplectic Geometry, and Geometric Analysis.

In Applied Mathematics, the Department has its focus on the areas of Applied Analysis, Mathematical Physics, Discrete and Computational Mathematics, and Probability and Statistics, and seeks to build excellence in these fields. Applications from those with an interest and ability to connect across units in the university to the advantage of research at the interface of mathematics and other disciplines are a top priority.

Candidates must have a $\mathrm{PhD}$ in Mathematics or a related field by the start date, strong record of research, and demonstrated evidence of excellent teaching ability. Qualified candidates should be committed to fostering diverse and inclusive environments as well as to promoting experiential learning, which are central to a Northeastern University education.

Responsibilities will include teaching undergraduate and graduate courses, mentoring students and conducting an independent research program.

Review of applications will begin immediately. Complete applications received by November 1, 2017 will be guaranteed full consideration. Additional applications will be considered until the position is filled.

To apply, please submit the documentation requested on the mathjobs.org web site. Applicants invited to interviews will be asked to complete a Northeastern University application on the appropriate website.

Northeastern University is an Equal Opportunity, Affirmative Action Educational Institution and Employer, Title IX University. Northeastern University particularly welcomes applications from minorities, women and persons with disabilities. Northeastern University is an E-Verify Employer.

00038

\section{NEBRASKA}

\section{University of Nebraska Department of Mathematics}

The Department of Mathematics at the University of Nebraska invites applications for the following position:

One Professor of Practice position, at the Assistant Professor Level, to take a leadership role in the Department's first-year mathematics program through calculus. Review of applications will begin December 1, 2017 and continue until a suitable candidate is found.

For more information about this position and information on how to apply for it, please go to: www.math.un1.edu/ department/jobs/.

The University of Nebraska-Lincoln is committed to a pluralistic campus community through affirmative action, equal opportunity, work-life balance, and dual careers. See www . un1 . edu/equity/ notice-nondiscrimination

00040

\section{PENNSYLVANIA}

\section{Penn State University Department of Mathematics}

The Department of Mathematics is seeking outstanding applicants for tenure and tenure-track faculty positions in all areas of Mathematics. A PhD is required. Applicants must complete the Penn State application electronically at psu.jobs/ job/74379 and must submit an application through Mathjobs.org www.mathjobs.org/jobs) with the following materials in order for the application to be complete: (1) Curriculum Vitae; (2) at least three references, one of which should address in detail the candidate's abilities as a teacher; (3) Publication List; (4) Research Statement; and (5) Teaching Statement. We encourage applications from individuals of diverse backgrounds. Review of applicants will begin November 15, 2017 and continue until the position is filled.

CAMPUS SECURITY CRIME STATISTICS: For more about safety at Penn State, and to review the Annual Security Report which contains information about crime statistics and other safety and security matters, please go to www.police.psu. edu/clery/, which will also provide you with detail on how to request a hard copy of the Annual Security Report.

Penn State is an equal opportunity, affirmative action employer, and is committed to providing employment opportunities to all qualified applicants without regard to race, color, religion, age, sex, sexual orientation, gender identity, national origin, disability or protected veteran status.

\section{Penn State University Eberly Research Fellows}

The Eberly College of Science at Penn State University invites nominees for the Eberly Research Fellowship program. Eberly Fellowships are designed to attract exceptional early career scientists to Penn State to enhance their career goals in the vibrant, highly collaborative environment of the Eberly College of Science and the broader STEM community of Penn State University. The Eberly College of Science which includes the Departments of Astronomy \& Astrophysics, Biology, Biochemistry \& Molecular Biology, Chemistry, Mathematics, Physics, and Statistics, ranks in the top 10 universities in the US and has annual research expenditures exceeding $\$ 100 \mathrm{M}$. Each of the seven departments is expecting to appoint one or more Eberly Fellows. Nominations for early career scientists with exceptional promise in basic research in physics, chemistry, biology, molecular biology, astronomy, mathematics, and statistics and/or applied research in health, energy, materials, or the environment are encouraged. Interdisciplinary as well as traditional disciplinary research is encouraged. Fellows who wish to also gain training and experience in teaching may elect to receive mentored teaching experience. Eberly Fellow advisors must hold their primary appointment in one of the seven departments of the Eberly College of Science. Co-advisors and crossdisciplinary research are also supported.

\section{Eligibility and appointment}

Applicants must be a current doctoral student or have received a doctoral degree in science, statistics, or mathematics within the past three years. Current doctoral students must have their doctoral degree prior to the start of their fellowship. Current doctoral students and postdoctoral fellows at Penn State are not eligible. Eberly Research Fellowships 
may be held from 1-3 years with annual appointments conditional on satisfactory performance. Fellows will receive a stipend of $\$ 65,000$ and $\$ 5,000$ per year in discretionary funds, which can be used for travel and other research expenses.

\section{Nomination and applications}

Nominations will be accepted from faculty advisors, graduate program chairs, department chairs, or others who can attest to the nominee's potential as a scientist. Nominations should include the nominees CV. Nominations of women and under-represented minorities are strongly encouraged. The nomination deadline is November 15 for appointments beginning 6-12 months later. Nominations should be sent to research-fe11ows@psu.edu.

The Eberly Research Fellowship Selection Committee will select the nominees who will then be requested to submit their applications by December 1, 2017. Applications will include (1) a biographical sketch - including publications, accepted, and submitted manuscripts, (2) three letters of reference including one from the doctoral advisor, (3) research statement summarizing research accomplishments and research that you intend to pursue at Penn State, (4) names of one or more potential faculty advisors among the faculty in the seven departments of the Eberly College of Science, Penn State University.

CAMPUS SECURITY CRIME STATISTICS: For more about safety at Penn State, and to review the Annual Security Report which contains information about crime statistics and other safety and security matters, please go to www. police.psu. edu/c7ery/, which will also provide you with detail on how to request a hard copy of the Annual Security Report.

Penn State is an equal opportunity, affirmative action employer, and is committed to providing employment opportunities to all qualified applicants without regard to race, color, religion, age, sex, sexual orientation, gender identity, national origin, disability or protected veteran status.

U.Ed. SCI 18-9

\section{TENNESSEE}

\section{Tenure-Track Assistant Professor Position at Vanderbilt University, Nashville, Tennessee}

The Department of Mathematics at Vanderbilt University invites applications for a tenure-track position (Assistant Professor) beginning fall 2018. Exceptional candidates from any area of pure or applied mathematics are encouraged to apply, but priority will be given to applicants in or related to one of the research areas of the department.

We are looking for individuals with an outstanding record in research and demonstrated excellence in teaching. A $\mathrm{PhD}$ degree is required. Qualified can- didates should submit their application materials electronically through the AMS website Mathjobs.org via the link www. mathjobs.org/jobs. Alternatively, application materials may be sent to app $7 y$. interfolio.com/43643. These materials should include a cover letter, a curriculum vitae, a publication list, a description of current and planned research, a teaching statement, at least four letters of recommendation, and the AMS Cover Sheet. One of the letters must discuss the applicant's teaching qualifications. Reference letter writers should be asked to submit their letters online through MathJobs.Org or through Interfolio. Evaluation of the applications will commence on November 1, 2017 and continue until the position is filled. For information about the Department of Mathematics at Vanderbilt University, please consult the web at as. vanderbi7t. edu/math/.

Vanderbilt University has a strong institutional commitment to recruiting and retaining an academically and culturally diverse community of faculty. Minorities, women, individuals with disabilities, and members of other underrepresented groups, in particular, are encouraged to apply. Vanderbilt is an Equal Opportunity/ Affirmative Action employer.

00035

\section{UTAH}

\section{University of Utah \\ College of Science}

Biophysics/Neuroscience/Chemical Biology Cluster, University of Utah. The proposed cluster comprises multiple tenure-track faculty positions to establish excellence in emerging interdisciplinary fields; departmental appointments may be in Physics, Biology, Chemistry, Mathematics or Biochemistry. A major goal is to strengthen both Cellular and Molecular Neuroscience and Biophysics, as well as to link Neuroscience to the Chemical Biology of Natural Products. All candidates are expected to demonstrate the ability to develop a vigorous, competitive and well-funded research program and will be evaluated on how their research potentially contributes to the long-term goal of excellence in interdisciplinary bridge areas. Applications by investigators, both experimental and theoretical, interested in macromolecular complexes on membranes are particularly encouraged. An undergraduate track in Neuroscience and Biophysics will be established within the College of Science, and faculty hired through this cluster are expected to participate in the development and teaching of innovative courses related to this initiative. We expect to make two or more faculty appointments in the 2017-2018 academic year, with additional appointments in subsequent years. While the openings are at the Assistant Professor level, exceptional senior candidates will be considered.

Assistant Professor applicants should upload an application letter with vita, a description of proposed research, and arrange for three letters of recommendation to be sent on their behalf.

Senior applicants should upload an application letter and CV. Applications should be uploaded in PDF format to utah . peopleadmin.com/postings/68311. Initial interview selections will begin as applications are received, but applications submitted at a later date will be considered as needed.

The University of Utah values candidates who have experience working with students from diverse backgrounds and possess a strong commitment to improving access to higher education for historically underrepresented groups. The University of Utah is an equal opportunity/ affirmative action employer and educator. Minorities, women, veterans and those with disabilities are strongly encouraged to apply. Veterans' Preference is extended to qualified veterans. Reasonable disability accommodations will be provided with adequate notice. For additional information about the University's commitment to equal opportunity and access, see www. utah.edu/nondiscrimination/.

00034

\section{University of Utah Department of Mathematics}

The Department of Mathematics at the University of Utah invites applications for the following positions:

- Full-time tenure-track or tenured appointments at the level of Assistant, Associate, or Full Professor in all areas of mathematics.

- Full-time tenure-track or tenured appointments at the level of Assistant, Associate, or Full Professor in all areas of statistics. These positions are part of a University-wide cluster hiring effort in statistics, with particular emphasis in mathematics, computer science, and bioengineering. Successful candidates will have strong interdisciplinary interests.

- Three-year Burgess, Tucker, and Wylie Assistant Professor Lecturer positions.

Please see our website at www.math. utah.edu/positions for information regarding available positions and application requirements. Applications must be completed through www . mathjobs .org/ jobs/Utah. Review of complete applications for tenure-track positions will begin on October 23, 2017, and will continue until the positions are filled. Completed applications for postdoctoral positions received before January 1, 2018, will receive full consideration.

The University of Utah is an Equal Opportunity/Affirmative Action employer and educator. Minorities, women, veterans, and those with disabilities are strongly en- 
couraged to apply. Veterans' preference is extended to qualified veterans. Reasonable disability accommodations will be provided with adequate notice. For additional information about the University's commitment to equal opportunity and access see: www. utah.edu/nondiscrimination/.

\section{LEBANON}

\section{American University of Beirut (AUB) Faculty positions: Mathematics}

The Department of Mathematics at the American University of Beirut (AUB) invites applications for tenure track positions in mathematics at the assistant professor level to begin August 15, 2018.

Only applicants holding a $\mathrm{PhD}$ in Mathematics, Statistics or Applied Mathematics and showing a strong commitment to teaching and research will be considered.

All interested applicants should send a letter of application with a current $\mathrm{CV}$, research and teaching statements, and arrange for three letters of reference to be addressed to Dean Nadia El Cheikh and sent through the Mathjobs website at www. mathjobs.org. Alternatively, applications may be sent by e-mail to as_dean@ aub.edu. 1b. Applicants should indicate whether they are planning to attend the AMS-MAA Joint Mathematics Meetings on January 10-13, 2018 in San Diego, California.

All applications and letters of reference should arrive by November 15, 2017. Applications will continue to be received after the deadline, but may not receive full consideration.

For more information on these positions, please visitwww . aub. edu. 1b/fas/ pages/academic-employment.aspx or the Department's website at www.aub. edu. 1b/fas/math.

The American University of Beirut is an Equal Opportunity Employer.

\section{TAIWAN}

\section{National Chengchi University Open Faculty Positions (Tenure Track)}

The Department of Mathematical Sciences at National Chengchi University in Taipei, Taiwan anticipates openings for several tenure-track faculty positions. The candidate must hold a doctoral degree in (Applied) Mathematics and be able to communicate in Chinese and English. For more information, please visit www . math. nccu. edu. tw/app/news . php? Sn=718.

\section{CO-AUTHOR SOUGHT}

Co-author sought to help finalize paper, "A Solution to the $3 x+1$ Problem"

I am seeking a professional mathematician to help me finalize a paper that sets forth a solution to the very difficult, longstanding, problem in number theory known as the $3 \mathrm{x}+1$ Problem. So far there have been well over 7,000 visits to the online paper; I have received not one claim of an error. It is reasonable to assume that at least 500 of those visits were by professional mathematicians, because that number is the increase in monthly visits that followed appearances of classified ads like this one in previous issues of the Notices.

I am not a professional mathematician (my degree is in computer science, and for many years I have been a researcher in the computer industry), and so I feel a co-author is essential.

The co-author will be expected to check the proof of each lemma, and the two proofs of the $3 x+1$ Conjecture each of which solves the Problem, and to help correct errors, and to offer suggestions for other improvements. He or she will also be expected to make contributions to content that he or she believes will enhance the paper, and, finally, to write to the editor of each journal to which the paper is submitted, stating his or her belief that the proofs are correct.

Anyone who is familiar with the Problem knows that very significant prestige will be gained by the co-author of the paper when it is published.

The paper is "A Solution to the $3 \mathrm{x}+1$ Problem," on occampress.com

Contact Peter Schorer at peteschorer@ gmai1.com.

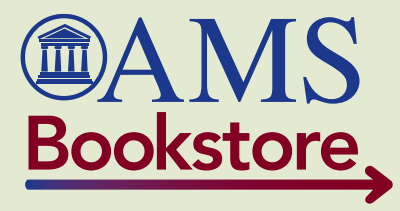

(c)

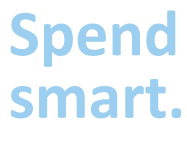

As an AMS

member, enjoy up to $20 \%$ off any of our titles. Visit the Bookstore regularly for special monthly sales and discounts.

\section{Search better.}

Our full listing of books is conveniently organized by multiple criteria and searchable by various advanced options to help you find exactly what you need.

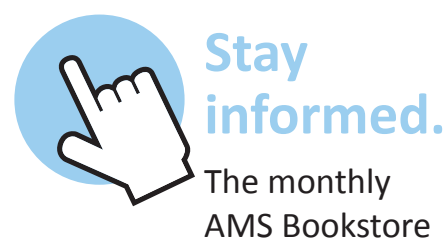

New Releases email offers many benefits to subscribers. Stay up-to-date on all AMS publications and sales.

Sign up today at:

ams.org/bookstore/ keepinformed

bookstore.ams.org

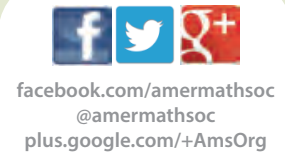



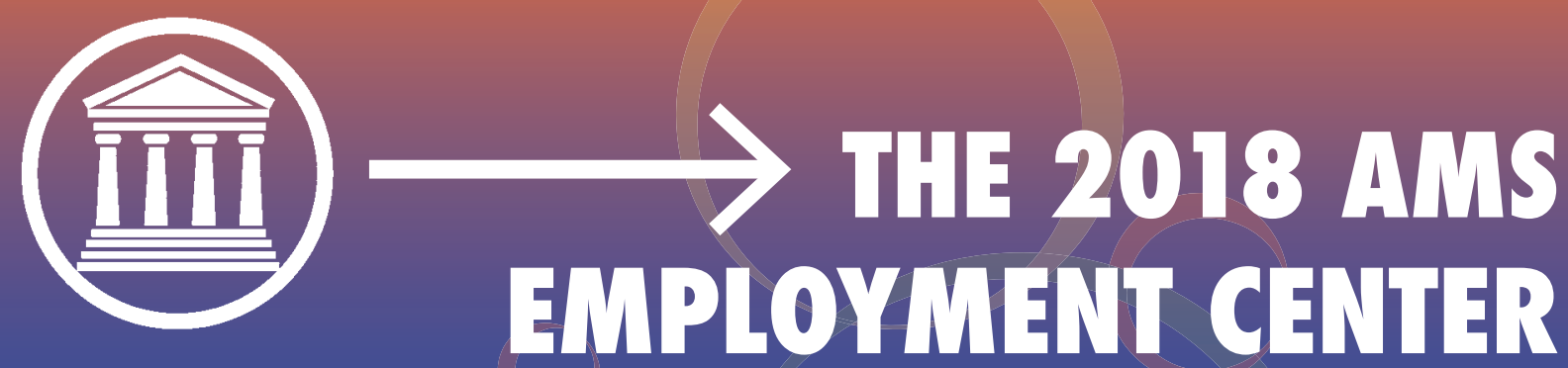

at the Joint Mathematics Meetings

Hall A

San Diego Convention Center, San Diego California January 10-13, 2018

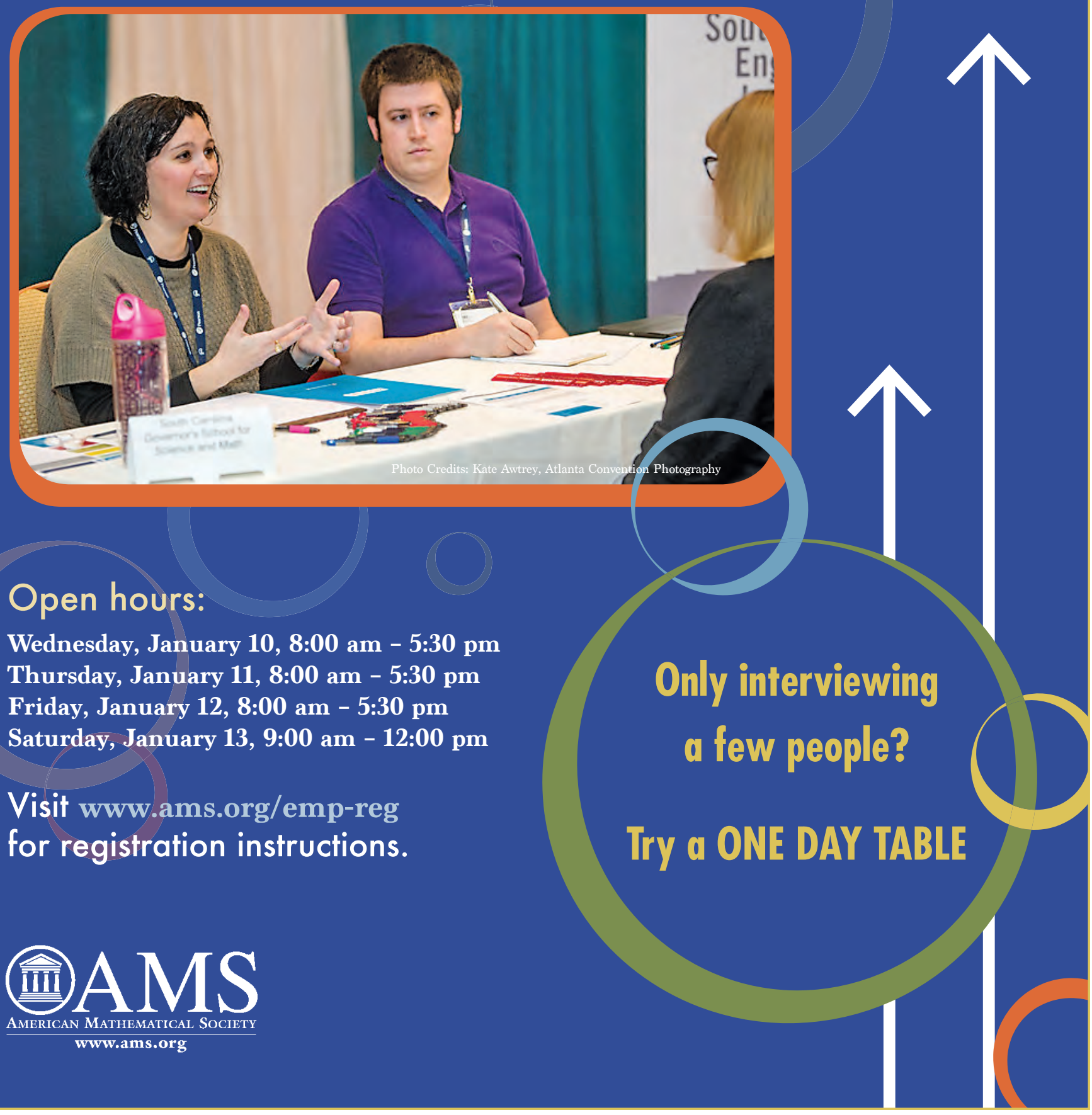




\section{MEETINGS \& CONFERENCES OF THE AMS}

\section{NOVEMBER TABLE OF CONTENTS}

The Meetings and Conferences section of the Notices gives information on all AMS meetings and conferences approved by press time for this issue. Please refer to the page numbers cited on this page for more detailed information on each event. Invited Speakers and Special Sessions are listed as soon as they are approved by the cognizant program committee; the codes listed are needed for electronic abstract submission. For some meetings the list may be incomplete. Information in this issue may be dated.
The most up-to-date meeting and conference information can be found online at: www.ams.org/meetings/.

Important Information About AMS Meetings: Potential organizers, speakers, and hosts should refer to page 75 in the January 2017 issue of the Notices for general information regarding participation in AMS meetings and conferences.

Abstracts: Speakers should submit abstracts on the easy-to-use interactive Web form. No knowledge of LTEX is necessary to submit an electronic form, although those who use IATEX may submit abstracts with such coding, and all math displays and similarily coded material (such as accent marks in text) must be typeset in LTEX. Visit www.ams.org/ cgi-bin/abstracts/abstract.pl/. Questions about abstracts may be sent to absinfo@ams.org. Close attention should be paid to specified deadlines in this issue. Unfortunately, late abstracts cannot be accommodated.

\section{MEETINGS IN THIS ISSUE}

\begin{tabular}{|c|c|c|}
\hline \multirow{2}{*}{ November $4-5$} & \multirow{2}{*}{$\begin{array}{l}2017 \\
\text { Riverside, California }\end{array}$} & \multirow[b]{2}{*}{ p. 1235} \\
\hline & & \\
\hline & 2018 & \\
\hline January 10-13 & San Diego, California & p. 1236 \\
\hline March 17-18 & Columbus, Ohio & p. 1239 \\
\hline April 14-15 & Portland, Oregon & p. 1241 \\
\hline April 14-15 & Nashville, Tennesse & p. 1242 \\
\hline April 21-22 & Boston, Massachusetts & p. 1242 \\
\hline June $11-14$ & $\begin{array}{l}\text { Shanghai, People's Republic } \\
\text { of China }\end{array}$ & c 1243 \\
\hline September 29-30 & Newark, Delaware & p. 1244 \\
\hline October 6-7 & Fayetteville, Arkansas & p. 1244 \\
\hline October 20-21 & Ann Arbor, Michigan & p. 1244 \\
\hline October 27-28 & San Francisco, California & p. 1245 \\
\hline
\end{tabular}

\section{9}

$\begin{array}{llr}\text { January 16-19 } & \text { Baltimore, Maryland } & \text { p. } 1245 \\ \text { March 15-17 } & \text { Auburn, Alabama } & \text { p. } 1245 \\ \text { March 22-24 } & \text { Honolulu, Hawaii } & \text { p. 1245-6 } \\ \text { June 10-13 } & \text { Quy Nhon City, Vietmam } & \text { p. } 1246 \\ \text { October 12-13 } & \text { Binghamton, New York } & \text { p. } 1246 \\ & & \\ & & \\ & & \end{array}$

January 15-18 Denver, Colorado

p. 1246

2021

January 6-9 Washington, DC p. 1246

See www. ams.org/meetings/ for the most up-to-date information on the meetings and conferences that we offer.

\section{ASSOCIATE SECRETARIES OF THE AMS}

Central Section: Georgia Benkart, University of WisconsinMadison, Department of Mathematics, 480 Lincoln Drive, Madison, WI 53706-1388; email: benkart@math.wisc.edu; telephone: 608-263-4283.

Eastern Section: Steven H. Weintraub, Department of Mathematics, Lehigh University, Bethlehem, PA 18015-3174; email: steve.weintraub@lehigh.edu; telephone: 610-758-3717.
Southeastern Section: Brian D. Boe, Department of Mathematics, University of Georgia, 220 D W Brooks Drive, Athens, GA 30602-7403, email: brian@math.uga.edu; telephone: 706-5422547.

Western Section: Michel L. Lapidus, Department of Mathematics, University of California, Surge Bldg., Riverside, CA 925210135; email: lapidus@math.ucr.edu; telephone: 951-827-5910. 


\section{Meetings \& Conferences of the AMS}

IMPORTANT INFORMATION REGARDING MEETINGS PROGRAMS: AMS Sectional Meeting programs do not appear

in the print version of the Notices. However, comprehensive and continually updated meeting and program informa-

tion with links to the abstract for each talk can be found on the AMS website. See www. ams. org/meetings/.

Final programs for Sectional Meetings will be archived on the AMS website accessible from the stated URL

Riverside, California

\section{University of California, Riverside}

\section{November 4-5, 2017}

Saturday - Sunday

\section{Meeting \# 1134}

Western Section

Associate secretary: Michel L. Lapidus

Announcement issue of Notices: September 2017

Program first available on AMS website: September 21, 2017

Issue of Abstracts: Volume 38, Issue 4

\section{Deadlines}

For organizers: Expired

For abstracts: Expired

The scientific information listed below may be dated. For the latest information, see www. ams.org/amsmtgs/ sectional.html.

\section{Invited Addresses}

Paul Balmer, University of California, Los Angeles, An invitation to tensor-triangular geometry.

Pavel Etingof, Massachusetts Institute of Technology, Double affine Hecke algebras and their applications.

Monica Vazirani, University of California, Davis, Combinatorics, Categorification, and Crystals.

\section{Special Sessions}

If you are volunteering to speak in a Special Session, you should send your abstract as early as possible via the abstract submission form found at www. ams . org/cgi-bin/ abstracts/abstract.p1.

Advances in Operator Algebras, Michael Hartglass, UC Riverside, Santa Clara University, and Chenxu Wen and Feng Xu, University of California, Riverside.

Algebraic Geometry, Humberto Diaz, Jose Gonzalez, and Ziv Ran, University of California, Riverside.
Algebraic and Combinatorial Structures in Knot Theory, Patricia Cahn, Smith College, and Sam Nelson, Claremont McKenna College.

Analysis and Geometry of Fractals, Erin Pearse, California Polytechnic State University, Goran Radunovic, University of Zagreb, and Tim Cobler, Fullerton College, California.

Applied Category Theory, John Baez, University of California, Riverside.

Characteristics of a Successful Mathematics Gateway Program, Sara Lapan, University of California, Riverside, Jeff Meyer, California State University, San Bernardino, and David Weisbart, University of California, Riverside.

Combinatorial Aspects of the Polynomial Ring, Sami Assaf and Dominic Searles, University of Southern California.

Combinatorial Representation Theory, Vyjayanthi Chari, University of California, Riverside, and Maria Monks Gillespie and Monica Vazirani, University of California, Davis.

Conservation Laws, Nonlinear Waves and Applications, Geng Chen, University of Kansas, Tien Khai Nguyen, North Carolina State University, and Qingtian Zhang, University of California, Davis.

Foundations of Quantum Theory, Jukka Virtanen, University of California, Los Angeles, and David Weisbart, University of California, Riverside.

Generalized Geometry, Daniele Grandini, Virginia State University, and Yat-Sun Poon, University of California, Riverside.

Geometric Analysis, Zhiqi Lu, University of California, Irvine, Jie Qing, University of California, Santa Cruz, Guofang Wei, University of California, Santa Barbara, and Qi Zhang, University of California, Riverside.

Geometric Partial Differential Equations and their Applications, Po-Ning Chen, University of California, Riverside, Henri Roesch, Duke University, and Richard M. Schoen and Xiangwen Zhang, University of California, Irvine.

Homotopy Theory, Jonathan Beardsley, University of Washington. 
Mathematical Fluid Mechanics, James P Kelliher and Lizheng Tao, University of California, Riverside.

Model Theory, Artem Chernikov, University of California, Los Angeles, and Isaac Goldbring, University of California, Irvine.

Non-Commutative Birational Geometry, Cluster Structures and Canonical Bases, Arkady Berenstein, University of Oregon, Eugene, Jacob Greenstein, University of California, Riverside, and Vladimir Retakh, Rutgers University.

Nonlinear Elliptic Differential and Integral Equations, Mathew Gluck, University of Oklahoma, and John Villavert, University of Texas, Rio Grande Valley.

Particle Methods and Nonlocal Partial Differential Equations, Katy Craig, University of California, Santa Barbara, and Franca Hoffman, University of Cambridge.

Preparing Students for American Mathematical Competitions, Adam Glesser, Phillip Ramirez, and Bogdan D. Suceava, California State University, Fullerton.

Random Matrices: Theory and Applications, Ioana Dumitriu, University of Washington, and Thomas Trogdon, University of California, Irvine.

Random and Deterministic Dynamical Systems, Nicolai Haydn, University of Southern California, Los Angeles.

Rational Cherednik Algebras and Categorification, Pavel Etingof, Massachusetts Institute of Technology, and Ivan Losev, Northeastern University.

Research in Mathematics by Early Career Graduate Students, Michael Bishop, Stefaan Delcroix, Marat Markin, Khang Tran, and Oscar Vega, California State University, Fresno.

Riemannian Manifolds of Non-Negative Sectional Curvature, Owen Dearricott, University of Melbourne, and Fernando Galaz-Garcia, Karlsruhe Institute of Technology.

Ring Theory and Related Topics (Celebrating the 75th Birthday of Lance W. Small), Jason Bell, University of Waterloo, Ellen Kirkman, Wake Forest University, and Susan Montgomery, University of Southern California.

Several Complex Variables, Bingyuan Liu and Bun Wong, University of California, Riverside.

Stochastic and Multi-scale Models in Mathematical Biology, Analysis and Simulations, Mark Alber, University of California, Riverside, and Bjorn Birnir, University of California, Santa Barbara.

Tensor Categories: Bridging Algebra, Topology, and Physics, Paul Bruillard, Pacific Northwest National Laboratory, Julia Plavnik, Texas A\&M University, and Henry Tucker, University of California, San Diego.

\section{San Diego, California}

San Diego Convention Center and San Diego Marriott Hotel and Marina

January 10-13,2018

Wednesday - Saturday

\section{Meeting \# 1135}

Joint Mathematics Meetings, including the 124th Annual Meeting of the AMS, 101st Annual Meeting of the Mathematical Association of America (MAA), annual meetings of the Association for Women in Mathematics (AWM) and the National Association of Mathematicians (NAM), and the winter meeting of the Association of Symbolic Logic (ASL), with sessions contributed by the Society for Industrial and Applied Mathematics (SIAM).

Associate secretary: Georgia Benkart

Announcement issue of Notices: October 2017

Program first available on AMS website: To be announced Issue of Abstracts: Volume 39, Issue 1

\section{Deadlines}

For organizers: Expired

For abstracts: Expired

The scientific information listed below may be dated. For the latest information, see www.ams.org/amsmtgs/ national.htm 1 .

\section{Joint Invited Addresses}

Gunnar Carlsson, Stanford University, Topological Modeling of Complex Data (AMS-MAA Invited Address).

Moon Duchin, Tufts University, Political Geometry: Voting districts,"compactness," and ideas about fairness (MAA-AMS-SIAM Gerald and Judith Porter Public Lecture).

André Neves, University of Chicago, Minimal surfaces, volume spectrum, and Morse index (AMS-MAA Invited Address).

Jill Pipher, Brown University, Nonsmooth boundary value problems (AWM-AMS Noether Lecture).

\section{AMS Invited Addresses}

Federico Ardila, San Francisco State University, Algebraic structures on polytopes.

Robert Bryant, Duke University, The Concept of Holonomy-its history and recent developments (AMS Retiring Presidential Address).

Ruth Charney, Brandeis University, Searching for Hyperbolicity.

Cynthia Dwork, Harvard University, Privavy in the Land of Plenty (AMS Josiah Willard Gibbs Lecture).

Dana Randall, Georgia Institute of Technology, Emergent phenomena in random structures and algorithms.

Edriss Titi, Texas A\&M University, The Navier-Stokes, Euler, and related equations.

Avi Wigderson, Princeton University, Alternate minimilization and scaling algorithms: theory, applications, and connections across mathematics and computer science (AMS Colloquium Lectures: Lecture I).

Avi Wigderson, Princeton University, Proving algebraic identities (AMS Colloquium Lectures: Lecture II).

Avi Wigderson, Princeton University, Proving analytic inequalities (AMS Colloquium Lectures: Lecture III). 


\section{AMS Special Sessions}

If you are volunteering to speak in a Special Session, you should send your abstract as early as possible via the abstract submission form found at jointmathematicsmeetings.org/meetings/abstracts/ abstract . $p 1$ ?type $=$ jmm.

Some sessions are cosponsored with other organizations. These are noted within the parenthesis at the end of each listing, where applicable.

A Showcase of Number Theory at Liberal Arts Colleges, Adriana Salerno, Bates College, and Lola Thompson, Oberlin College.

Accelerated Advances in Mathematical Fractional Programming, Ram Verma, International Publications USA, and Alexander Zaslavski, Israel Institute of Technology.

Advances in Applications of Differential Equations to Disease Modeling, Libin Rong, Oakland University, Elissa Schwartz, Washington State University, and Naveen K. Vaidya, San Diego State University.

Advances in Difference, Differential, and Dynamic Equations with Applications, Elvan Akin, Missouri University S\&T, and John Davis, Baylor University.

Advances in Operator Algebras, Marcel Bischoff, Vanderbilt University, Ian Charlesworth, University of California, Los Angeles, Brent Nelson, University of California, Berkeley, and Sarah Reznikoff, Kansas State University.

Advances in Operator Theory, Operator Algebras, and Operator Semigroups, Asuman G. Aksoy, Claremont McKenna College, Zair Ibragimov, California State University, Fullerton, Marat Markin, California State University, Fresno, and Ilya Spitkovsky, New York University, Abu Dhabi.

Algebraic, Analytic, and Geometric Aspects of Integrable Systems, Painlevé Equations, and Random Matrices, Vladimir Dragovic, University of Texas at Dallas, Anton Dzhamay, University of Northern Colorado, and Sevak Mkrtchyan, University of Rochester.

Algebraic, Discrete, Topological and Stochastic Approaches to Modeling in Mathematical Biology, Olcay Akman, Illinois State University, Timothy D. Comar, Benedictine University, Daniel Hrozencik, Chicago State University, and Raina Robeva, Sweet Briar College.

Alternative Proofs in Mathematical Practice, John W. Dawson, Jr., Pennsylvania State University, York.

Analysis of Fractional, Stochastic, and Hybrid Dynamic Systems, John R. Graef, University of Tennessee at Chattanooga, Gangaram S. Ladde, University of South Florida, and Aghalaya S. Vatsala, University of Louisiana at Lafayette.

Analysis of Nonlinear Partial Differential Equations and Applications, Tarek M. Elgindi, University of California, San Diego, and Edriss S. Titi, Texas A\&M University and Weizmann Institute of Science.

Applied and Computational Combinatorics, Torin Greenwood, Georgia Institute of Technology, and Jay Pantone, Dartmouth College.
Arithmetic Dynamics, Robert L. Benedetto, Amherst College, Benjamin Hutz, Saint Louis University, Jamie Juul, Amherst College, and Bianca Thompson, Harvey Mudd College.

Beyond Planarity: Crossing Numbers of Graphs (a Mathematics Research Communities Session), Axel Brandt, Davidson College, Garner Cochran, University of South Carolina, and Sarah Loeb, College of William and Mary.

Bifurcations of Difference Equations and Discrete Dynamical Systems, Arzu Bilgin and Toufik Khyat, University of Rhode Island.

Boundaries for Groups and Spaces, Joseph Maher, CUNY College of Staten Island, and Genevieve Walsh, Tufts University.

Combinatorial Commutative Algebra and Polytopes, Robert Davis, Michigan State University, and Liam Solus, KTH Royal Institute of Technology.

Combinatorics and Geometry, Federico Ardila, San Francisco State University, Anastasia Chavez, MSRI and University of California, Davis, and Laura Escobar, University of Illinois Urbana Champaign.

Commutative Algebra in All Characteristics, Neil Epstein, George Mason University, Karl Schwede, University of Utah, and Janet Vassilev, University of New Mexico.

Computational Combinatorics and Number Theory, Jeremy F. Alm, Lamar University, and David Andrews and Rob Hochberg, University of Dallas.

Connections in Discrete Mathematics: Graphs, Hypergraphs, and Designs, Amin Bahmanian, Illinois State University, and Theodore Molla, University Illinois UrbanaChampaign.

Differential Geometry, Vincent B. Bonini and Joseph E. Borzellino, Cal Poly San Luis Obispo, Bogdan D. Suceava, California State University, Fullerton, and Guofang Wei, University of California, Santa Barbara.

Diophantine Approximation and Analytic Number Theory in Honor of Jeffrey Vaaler, Shabnam Akhtari, University of Oregon, Lenny Fukshansky, Claremont McKenna College, and Clayton Petsche, Oregon State University.

Discrete Dynamical Systems and Applications, E. Cabral Balreira, Saber Elaydi, and Eddy Kwessi, Trinity University.

Discrete Neural Networking and Applications, Murat Adivar, Fayetteville State University, Michael A. Radin, Rochester Institute of Technology, and Youssef Raffoul, University of Dayton.

Dynamical Algebraic Combinatorics, James Propp, University of Massachusetts, Lowell, Tom Roby, University of Connecticut, Jessica Striker, North Dakota State University, and Nathan Williams, University of California Santa Barbara.

Dynamical Systems with Applications to Mathematical Biology, Guihong Fan, Columbus State University, Jing Li, California State University Northridge, and Chunhua Shan, University of Toledo.

Dynamical Systems: Smooth, Symbolic, and Measurable (a Mathematics Research Communities Session), Kathryn 
Lindsey, Boston College, Scott Schmieding, Northwestern University, and Kurt Vinhage, University of Chicago.

Emergent Phenomena in Discrete Models, Dana Randall, Georgia Institute of Technology, and Andrea Richa, Arizona State University.

Emerging Topics in Graphs and Matrices, Sudipta Mallik, Northern Arizona University, Keivan Hassani Monfared, University of Calgary, and Bryan Shader, University of Wyoming.

Ergodic Theory and Dynamical Systems-to Celebrate the Work of Jane Hawkins, Julia Barnes, Western Carolina University, Rachel Bayless, Agnes Scott College, Emily Burkhead, Duke University, and Lorelei Koss, Dickinson College.

Extremal Problems in Approximations and Geometric Function Theory, Ram Mohapatra, University of Central Florida.

Financial Mathematics, Actuarial Sciences, and Related Fields, Albert Cohen, Michigan State University, Nguyet Nguyen, Youngstown State University, Oana Mocioalca, Kent State University, and Thomas Wakefield, Youngstown State University.

Fractional Difference Operators and Their Application, Christopher S. Goodrich, Creighton Preparatory School, and Rajendra Dahal, Coastal Carolina University.

Free Convexity and Free Analysis, J. William Helton, University of California, San Diego, and Igor Klep, University of Auckland.

Geometric Analysis, Davi Maximo, University of Pennsylvania, Lu Wang, University of Wisconsin-Madison, and Xin Zhou, University of California Santa Barbara.

Geometric Analysis and Geometric Flows, David Glickenstein, University of Arizona, and Brett Kotschwar, Arizona State University.

History of Mathematics, Sloan Despeaux, Western Carolina University, Jemma Lorenat, Pitzer College, Clemency Montelle, University of Canterbury, Daniel Otero, Xavier University, and Adrian Rice, Randolph-Macon College.

Homotopy Type Theory (a Mathematics Research Communities Session), Simon Cho, University of Michigan, Liron Cohen, Cornell University, and Edward Morehouse, Wesleyan University.

If You Build It They Will Come: Presentations by Scholars in the National Alliance for Doctoral Studies in the Mathematical Sciences, Edray Goins and David Goldberg, Purdue University, and Phil Kutzko, University of Iowa.

Interactions of Inverse Problems, Signal Processing, and Imaging, M. Zuhair Nashed, University of Central Florida, Willi Freeden, University of Kaiserslautern, and Otmar Scherzer, University of Vienna.

Markov Chains, Markov Processes and Applications, Alan Krinik and Randall J. Swift, California State Polytechnic University.

Mathematical Analysis and Nonlinear Partial Differential Equations, Hongjie Dong, Brown University, Peiyong Wang, Wayne State University, and Jiuyi Zhu, Louisiana State University.
Mathematical Fluid Mechanics: Analysis and Applications, Zachary Bradshaw and Aseel Farhat, University of Virginia.

Mathematical Information in the Digital Age of Science, Patrick Ion, University of Michigan, Olaf Teschke, zbMath Berlin, and Stephen Watt, University of Waterloo.

Mathematical Methods in Genomics, David Koslicki, Oregon State University.

Mathematical Modeling and Analysis of Infectious Diseases, Kazuo Yamazaki, University of Rochester.

Mathematical Modeling of Natural Resources, Shandelle M. Henson, Andrews University, and Natali Hritonenko, Prairie View A\&M University.

Mathematical Modeling, Analysis and Applications in Population Biology, Yu Jin, University of Nebraska-Lincoln, and Ying Zhou, Lafayette College.

Mathematical Problems in Ocean Wave Modeling and Fluid Mechanics, Christopher W. Curtis, San Diego State University, and Katie Oliveras, Seattle University.

Mathematical Relativity and Geometric Analysis, James Dilts and Michael Holst, University of California, San Diego.

Mathematics Research from the SMALL Undergraduate Research Program, Colin Adams, Frank Morgan, and Cesar E. Silva, Williams College.

Mathematics of Gravitational Wave Science, Andrew Gillette and Nikki Holtzer, University of Arizona.

Mathematics of Quantum Computing and Topological Phases of Matter, Paul Bruillard, Pacific Northwest National Laboratory, David Meyer, University of California San Diego, and Julia Plavnik, Texas A\&M University.

Metric Geometry and Topology, Christine Escher, Oregon State University, and Catherine Searle, Wichita State University.

Modeling in Differential Equations-High School, TwoYear College, Four-Year Institution, Corban Harwood, George Fox University, William Skerbitz, Wayzata High School, Brian Winkel, SIMIODE, and Dina Yagodich, Frederick Community College.

Multi-scale Modeling with PDEs in Computational Science and Engineering:Algorithms, Simulations, Analysis, and Applications, Salim M. Haidar, Grand Valley State University.

Network Science, David Burstein, Swarthmore College, Franklin Kenter, United States Naval Academy, and Feng Shi, University of North Carolina at Chapel Hill.

New Trends in Celestial Mechanics, Richard Montgomery, University of California Santa Cruz, and Zhifu Xie, University of Southern Mississippi.

Nilpotent and Solvable Geometry,I, Michael Jablonski, University of Oklahoma, Megan Kerr, Wellesley College, and Tracy Payne, Idaho State University.

Noncommutative Algebras and Noncommutative Invariant Theory, Ellen Kirkman, Wake Forest University, and James Zhang, University of Washington.

Nonlinear Evolution Equations of Quantum Physics and Their Topological Solutions, Stephen Gustafson, University of British Columbia, Israel Michael Sigal, University of Toronto, and Avy Soffer, Rutgers University. 
Novel Methods of Enhancing Success in Mathematics Classes, Ellina Grigorieva, Texas Womans University, and Natali Hritonenko, Prairie View A\&M University.

Open and Accessible Problems for Undergraduate Research, Michael Dorff, Brigham Young University, Allison Henrich, Seattle University, and Nicholas Scoville, Ursinus College.

Operators on Function Spaces in One and Several Variables, Catherine Bénéteau, University of South Florida, and Matthew Fleeman and Constanze Liaw, Baylor University.

Orthogonal Polynomials and Applications, Abey LopezGarcia, University of South Alabama, and Xiang-Sheng Wang, University of Louisiana at Lafayette.

Orthogonal Polynomials, Quantum Probability, and Stochastic Analysis, Julius N. Esunge, University of Mary Washington, and Aurel I. Stan, Ohio State University.

Quantum Link Invariants, Khovanov Homology, and Low-dimensional Manifolds, Diana Hubbard, University of Michigan, and Christine Ruey Shan Lee, University of Texas at Austin.

Quaternions, Terrence Blackman, Medgar Evers College, City University of New York, and Johannes Familton and Chris McCarthy, Borough of Manhattan Community College, City University of New York.

Recent Trends in Analysis of Numerical Methods of Partial Differential Equations, Sara Pollock, Wright State University, and Leo Rebholz, Clemson University.

Research by Postdocs of the Alliance for Diversity in Mathematics, Aloysius Helminck, University of HawaiiManoa, and Michael Young, Iowa State University.

Research from the Rocky Mountain-Great Plains Graduate Research Workshop in Combinatorics, Michael Ferrara, University of Colorado Denver, Leslie Hogben, Iowa State University, Paul Horn, University of Denver, and Tyrrell McAllister, University of Wyoming.

Research in Mathematics by Early Career Graduate Students, Michael Bishop, Marat Markin, Khang Tran, and Oscar Vega, California State University, Fresno.

Research in Mathematics by Undergraduates and Students in Post-Baccalaureate Programs, Tamas Forgacs, CSU Fresno, Darren A. Narayan, Rochester Institute of Technology, and Mark David Ward, Purdue University (AMS-MAA-SIAM).

Set Theory, Logic and Ramsey Theory, Andrés Caicedo, Mathematical Reviews, and José Mijares, University of Colorado, Denver (AMS-ASL).

Set-theoretic Topology (Dedicated to Jack Porter in Honor of 50 Years of Dedicated Research), Nathan Carlson, California Lutheran University, Jila Niknejad, University of Kansas, and Lynne Yengulalp, University of Dayton.

Special Functions and Combinatorics (in honor of Dennis Stanton's 65th birthday), Susanna Fishel, Arizona State University, Mourad Ismail, University of Central Florida, and Vic Reiner, University of Minnesota.

Spectral Theory, Disorder and Quantum Physics, Rajinder Mavi and Jeffery Schenker, Michigan State University.
Stochastic Processes, Stochastic Optimization and Control, Numerics and Applications, Hongwei Mei, University of Central Florida, Zhixin Yang and Quan Yuan, Ball State University, and Guangliang Zhao, GE Global Research.

Strengthening Infrastructures to Increase Capacity Around K-20 Mathematics, Brianna Donaldson, American Institute of Mathematics, William Jaco and Michael Oehrtman, Oklahoma State University, and Levi Patrick, Oklahoma State Department of Education.

Structure and Representations of Hopf Algebras: a Session in Honor of Susan Montgomery, Siu-Hung Ng, Louisiana State University, and Lance Small and Henry Tucker, University of California, San Diego.

Theory, Practice, and Applications of Graph Clustering, David Gleich, Purdue University, and Jennifer Webster and Stephen J. Young, Pacific Northwest National Laboratory.

Topological Data Analysis, Henry Adams, Colorado State University, Gunnar Carlsson, Stanford University, and Mikael Vejdemo-Johansson, CUNY College of Staten Island.

Topological Graph Theory: Structure and Symmetry, Jonathan L. Gross, Columbia University, and Thomas W. Tucker, Colgate University.

Visualization in Mathematics: Perspectives of Mathematicians and Mathematics Educators, Karen Allen Keene, North Carolina State University, and Mile Krajcevski, University of South Florida.

Women in Symplectic and Contact Geometry and Topology, Bahar Acu, Northwestern University, Ziva Myer, Duke University, and Yu Pan, Massachusetts Institute of Technology (AMS-AWM).

\section{Columbus, Ohio \\ Ohio State University}

March 17-18, 2018

Saturday - Sunday

Meeting \#1136

Central Section

Associate secretary: Georgia Benkart

Announcement issue of Notices: December 2017

Program first available on AMS website: January 31, 2018 Issue of Abstracts: Volume 39, Issue 2

\section{Deadlines}

For organizers: Expired

For abstracts: January 22, 2018

The scientific information listed below may be dated. For the latest information, see www.ams.org/amsmtgs/ sectional.html.

\section{Invited Addresses}

Aaron Brown, University of Chicago, Title to be announced. 
Tullia Dymarz, University of Wisconsin-Madison, Title to be announced.

June Huh, Institute for Advanced Study, Title to be announced.

\section{Special Sessions}

If you are volunteering to speak in a Special Session, you should send your abstract as early as possible via the abstract submission form found at www. ams . org/cgi-bin/ abstracts/abstract.p1.

Advances in Integral and Differential Equations (Code: SS 26A), Jeffrey T. Neugebauer, Eastern Kentucky University, and Min Wang, Rowan University.

Algebraic Coding Theory and Applications (Code: SS 27A), Heide Gluesing-Luerssen, University of Kentucky, Christine A. Kelley, University of Nebraska-Lincoln, and Steve Szabo, Eastern Kentucky University.

Algebraic Combinatorics: Association Schemes, Finite Geometry, and Related Topics (Code: SS 15A), Sung Y. Song, Iowa State University, and Bangteng $\mathrm{Xu}$, Eastern Kentucky University.

Algebraic Curves and Their Applications (Code: SS 17A), Artur Elezi, American University, Monika Polak, Maria Curie-Sklodowska University (Poland) and University of Information Science and Technology (Mac, and Tony Shaska, Oakland University.

Algebraic and Combinatorial Aspects of Tropical Geometry (Code: SS 11A), Maria Angelica Cueto, Ohio State University, Yoav Len, University of Waterloo, and Martin Ulirsch, University of Michigan.

Algebraic, Combinatorial, and Quantum Invariants of Knots and Manifolds (Code: SS 6A), Cody Armond, Ohio State University, Mansfield, Micah Chrisman, Monmouth University, and Heather Dye, McKendree University.

Analytical and Computational Advances in Mathematical Biology Across Scales (Code: SS 30A), Veronica Ciocanel and Alexandria Volkening, Mathematical Biosciences Institute.

Categorical, Homological and Combinatorial Methods in Algebra (Celebrating the 80th birthday of S. K. Jain) (Code: SS 28A), Pedro A. Guil Asensio, University of Murcia, Ivo Herzog, Ohio State University, Andre Leroy, University of Artois, and Ashish K. Srivastava, Saint Louis University.

Coherent Structures in Interfacial Flows (Code: SS 14A), Benjamin Akers and Jonah Reeger, Air Force Institute of Technology.

Commutative and Combinatorial Algebra (Code: SS 18A), Jennifer Biermann, Hobart and William Smith Colleges, and Kuei-Nuan Lin, Penn State University, Greater Allegheny.

Convex Bodies in Algebraic Geometry and Representation Theory (Code: SS 20A), Dave Anderson, Ohio State University, and Kiumars Kaveh, University of Pittsburgh.

Differential Equations and Applications (Code: SS 8A), King-Yeung Lam and Yuan Lou, Ohio State University, and Qiliang Wu, Michigan State University.

Function Spaces, Operator Theory, and Non-Linear Differential Operators (Code: SS 21A), David Cruz-Uribe,
University of Alabama, and Osvaldo Mendez, University of Texas.

Geometric Methods in Shape Analysis (Code: SS 10A), Sebastian Kurtek and Tom Needham, Ohio State University. Graph Theory (Code: SS 5A), John Maharry, Ohio State University, Yue Zhao, University of Central Florida, and Xiangqian Zhou, Wright State University.

Homological Algebra (Code: SS 4A), Ela Celikbas and Olgur Celikbas, West Virginia University.

Homotopy Theory (Code: SS 29A), Ernest Fontes, John E. Harper, Crichton Ogle, and Gabriel Valenzuela, Ohio State University.

Lefschetz Properties (Code: SS 24A), Juan Migliore, University of Notre Dame, and Uwe Nagel, University of Kentucky.

Mathematical Modeling of Neuronal Networks (Code: SS 36A), Janet Best, Ohio State University, Alicia Prieto Langarica, Youngstown State University, and Pamela B. Pyzza, Ohio Wesleyan University.

Multiplicative Ideal Theory and Factorization (in honor of Tom Lucas retirement) (Code: SS 7A), Evan Houston, University of North Carolina, Charlotte, and Alan Loper, Ohio State University.

Noncommutative Algebra and Noncommutative Algebraic Geometry (Code: SS 16A), Jason Gaddis, Miami University, and Robert Won, Wake Forest University.

Nonlinear Evolution Equations (Code: SS 9A), John Holmes and Feride Tiglay, Ohio State University.

Nonlinear Waves and Patterns (Code: SS 19A), Anna Ghazaryan, Miami University, Stephane Lafortune, College of Charleston, and Vahagn Manukian and Alin Pogan, Miami University.

Parameter Analysis and Estimation in Applied Dynamical Systems (Code: SS 35A), Adriana Dawes, The Ohio State University, and Reginald L. McGee, Mathematical Biosciences Institute.

Probabilistic and Extremal Graph Theory (Code: SS 32A), Louis DeBiasio and Tao Jiang, Miami University.

Probability in Convexity and Convexity in Probability (Code: SS 2A), Elizabeth Meckes, Mark Meckes, and Elisabeth Werner, Case Western Reserve University.

Quantum Symmetries (Code: SS 3A), David Penneys, The Ohio State University, and Julia Plavnik, Texas A \& M University.

Recent Advances in Approximation Theory and Operator Theory (Code: SS 1A), Jan Lang and Paul Nevai, The Ohio State University.

Recent Advances in Finite Element Methods for Partial Differential Equations (Code: SS 31A), Ching-shan Chou, Yukun Li, and Yulong Xing, The Ohio State University.

Recent Advances in Packing (Code: SS 23A), Joseph W. Iverson, University of Maryland, John Jasper, South Dakota State University, and Dustin G. Mixon, The Ohio State University.

Recent Development of Nonlinear Geometric PDEs (Code: SS 12A), Bo Guan, Ohio State University, Qun Li, Wright State University, Xiangwen Zhang, University of California, Irvine, and Fangyang Zheng, Ohio State University. 
Several Complex Variables (Code: SS 13A), Liwei Chen, Kenneth Koenig, and Liz Vivas, Ohio State University.

Stochastic Analysis in Infinite Dimensions (Code: SS 22A), Parisa Fatheddin, Air Force Institute of Technology, and Arnab Ganguly, Louisiana State University.

Structure and Representation Theory of Finite Groups (Code: SS 33A), Justin Lynd, University of Louisiana at Lafayette, and Hung Ngoc Nguyen, University of Akron.

Symmetry in Differential Geometry (Code: SS 34A), Samuel Lin, Dartmouth College, Barry Minemyer, Bloomsburg University, and Ben Schmidt, Michigan State University.

The Mathematics of Phylogenetics (Code: SS 25A), Colby Long, Mathematical Biosciences Institute.

Topology and Geometry in Data Analysis (Code: SS 37A), Sanjeevi Krishnan and Facundo Memoli, Ohio State University.

\section{Portland, Oregon}

\section{Portland State University}

April 14-15, 2018

Saturday - Sunday

\section{Meeting \# 1137}

Western Section

Associate secretary: Michel L. Lapidus

Announcement issue of Notices: January 2018

Program first available on AMS website: February 15, 2018

Issue of Abstracts: Volume 39, Issue 2

\section{Deadlines}

For organizers: Expired

For abstracts: February 6, 2018

The scientific information listed below may be dated. For the latest information, see www. ams.org/amsmtgs/ sectional.htm1.

\section{Invited Addresses}

Sándor Kovács, University of Washington, Seattle, Title to be announced.

Elena Mantovan, California Institute of Technology, Title to be announced.

Dimitri Shlyakhtenko, University of California, Los Angeles, Title to be announced.

\section{Special Sessions}

If you are volunteering to speak in a Special Session, you should send your abstract as early as possible via the abstract submission form found at www. ams . org/cgi-bin/ abstracts/abstract.p1.

Algebraic Geometry and its Connections (Code: SS 9A), Sándor Kovács, University of Washington, Seattle, and Karl Schwede, University of Utah, Salt Lake City.

Algebraic Topology (Code: SS 23A), Angélica Osorno,

Reed College, and Dev Sinha, University of Oregon

Algebraic and Combinatorial Structures in Knot Theory

(Code: SS 3A), Allison Henrich, Seattle University, Inga
Johnson, Willamette University, and Sam Nelson, Claremont McKenna College.

Automorphisms of Riemann Surfaces and Related Topics (Code: SS 14A), S. Allen Broughton, Rose-Hulman Institute of Technology, Mariela Carvacho, Universidad Tecnica Federico Santa Maria, Anthony Weaver, Bronx Community College, the City University of New York, and Aaron Wootton, University of Portland.

Biomathematics - Progress and Future Directions (Code: SS 4A), Hannah Callender Highlander, University of Portland, Peter Hinow, University of Wisconsin-Milwaukee, and Deena Schmidt, University of Nevada, Reno.

Commutative Algebra (Code: SS 5A), Adam Boocher, University of Utah, and Irena Swanson, Reed College.

Complex Analysis and Applications (Code: SS 11A), Malik Younsi, University of Hawaii Manoa.

Differential Geometry (Code: SS 19A), Christine Escher, Oregon State University, and Catherine Searle, Wichita State University.

Forest Modeling (Code: SS 20A), Gatziolis Demetrios, Pacific Northwest Research Station, US Forest Service, and Nikolay Strigul, Washington State University, Vancouver.

General Relativity and Geometric Analysis (Code: SS 13A), Paul T. Allen, Lewis \& Clark College, Jeffrey Jauregui, Union College, and Iva Stavrov Allen, Lewis \& Clark College.

Geometric Measure Theory and Partial Differential Equations (Code: SS 12A), Mark Allen, Brigham Young University, and Spencer Becker-Kahn and Mariana Smit Vega Garcia, University of Washington.

Inverse Problems (Code: SS 2A), Hanna Makaruk, Los Alamos National Laboratory (LANL), and Robert Owczarek, University of New Mexico, Albuquerque \& Los Alamos.

Mock Modular and Quantum Modular Forms (Code: SS 24A), Holly Swisher, Oregon State University, and Stephanie Treneer, Western Washington University.

Modeling, Analysis, and Simulation of PDEs with Multiple Scales, Interfaces, and Coupled Phenomena (Code: SS 17A), Malgorzata Peszynska, Oregon State University.

Moduli Spaces (Code: SS 22A), Renzo Cavalieri, Colorado State University, and Damiano Fulghesu, Minnesota State University Moorhead.

Motivic homotopy theory (Code: SS 6A), Daniel Dugger, University of Oregon, and Kyle Ormsby, Reed College.

Noncommutative Algebraic Geometry and Related Topics (Code: SS 16A), Jesse Levitt, University of Southern California, Hans Nordstrom, University of Portland, and Xinting Wang, Temple University.

Nonsmooth Optimization and Applications(Dedicated to Prof. B. S. Mordukhovich on the occasion of his 70th birthday) (Code: SS 7A), Mau Nam Nguyen, Portland State University, Hung M. Phan, University of Massachusetts Lowell, and Shawn Xianfu Wang, University of British Columbia.

Numerical Methods for Partial Differential Equations (Code: SS 22A), Brittany A. Erickson, Portland State University, and Jeffrey S. Ovall, Portland State University. 
Pattern Formation in Crowds, Flocks, and Traffic (Code: SS 1A), J. J. P. Veerman, Portland State University, Alethea Barbaro, Case Western Reserve University, and Bassam Bamieh, UC Santa Barbara.

Recent Advances in Actuarial Mathematics (Code: SS 18A), Sooie-Hoe Loke, Central Washington University, and Enrique Thomann, Oregon State University.

Spectral Theory (Code: SS 8A), Jake Fillman, Virginia Tech, and Milivoje Lukic, Rice University.

Teaching and Learning in Undergraduate Mathematics (Code: SS 15A), Natalie LF Hobson, Sonoma State University, and Elise Lockwood, Oregon State University.

Wavelets, Frames, and Related Expansions (Code: SS 10A), Marcin Bownik, University of Oregon, and Darrin Speegle, Saint Louis University.

\section{Nashville, Tennessee}

\section{Vanderbilt University}

April 14-15, 2018

Saturday - Sunday

\section{Meeting \#1138}

Southeastern Section

Associate secretary: Brian D. Boe

Announcement issue of Notices: January 2018

Program first available on AMS website: February 22, 2018

Issue of Abstracts: Volume 39, Issue 2

\section{Deadlines}

For organizers: Expired

For abstracts: February 13, 2018

The scientific information listed below may be dated. For the latest information, see www.ams.org/amsmtgs / sectional.htm1.

\section{Invited Addresses}

Andrea Bertozzi, University of California Los Angeles, Title to be announced (Erdős Memorial Lecture).

J. M. Landsberg, Texas A \& M University, Title to be announced.

Jennifer Morse, University of Virginia, Title to be announced.

Kirsten Wickelgren, Georgia Institute of Technology, Title to be announced.

\section{Special Sessions}

If you are volunteering to speak in a Special Session, you should send your abstract as early as possible via the abstract submission form found at www . ams . org/cgi-bin/ abstracts/abstract.p1.

Advances in Operator Algebras (Code: SS 7A), Scott Atkinson, Dietmar Bisch, Vaughan Jones, and Jesse Peterson, Vanderbilt University.

Commutative Algebra (Code: SS 8A), Florian Enescu and Yongwei Yao, Georgia State University.
Difference Equations and Applications (Code: SS 2A), Michael A. Radin, Rochester Institute of Technology, and Youssef Raffoul, University of Dayton, Ohio.

Function Spaces and Operator Theory (Code: SS 9A), Cheng Chu and Dechao Zheng, Vanderbilt University.

Harmonic Analysis, Functional Analysis, and Their Applications (Code: SS 11A), Akram Aldroubi and Keaton Hamm, Vanderbilt University, Michael Worthington, Georgia Institute of Technology, and Alex Powell, Vanderbilt University.

Interactions between Geometry, Group Theory and Dynamics (Code: SS 13A), Jayadev Athreya, University of Washington, and Caglar Uyanik and Grace Work, Vanderbilt University.

Mathematical Chemistry (Code: SS 10A), Hua Wang, Georgia Southern University.

Matroids and Related Structures (Code: SS 5A), Carolyn Chun, United States Naval Academy, Deborah Chun and Tyler Moss, West Virginia University Institute of Technology, and Jakayla Robbins, Vanderbilt University.

Probabilistic Models in Mathematical Physics (Code: SS 6A), Robert Buckingham, University of Cincinnati, SeungYeop Lee, University of South Florida, and Karl Liechty, DePaul University.

Quantization for Probability Distributions and Dynamical Systems (Code: SS 1A), Mrinal Kanti Roychowdhury, University of Texas Rio Grande Valley.

Recent Advances in Mathematical Biology (Code: SS 12A), Glenn Webb and Yixiang Wu, Vanderbilt University. Selected Topics in Graph Theory (Code: SS 3A), Songling Shan, Vanderbilt University, and David Chris Stephens and Dong Ye, Middle Tennessee State University.

Structural Graph Theory (Code: SS 4A), Joshua Fallon, Louisiana State University, and Emily Marshall, Arcadia University.

\section{Boston, Massachusetts}

\section{Northeastern University}

April 21-22, 2018

Saturday - Sunday

\section{Meeting \# 1139}

Eastern Section

Associate secretary: Steven H. Weintraub

Announcement issue of Notices: January 2018

Program first available on AMS website: March 1, 2018

Issue of Abstracts: Volume 39, Issue 2

\section{Deadlines}

For organizers: Expired

For abstracts: February 20, 2018 
The scientific information listed below may be dated. For the latest information, see www.ams.org/amsmtgs/ sectional.html.

\section{Invited Addresses}

Jian Ding, University of Chicago, Title to be announced.

Edward Frenkel, University of California, Berkeley, Title to be announced (Einstein Public Lecture in Mathematics).

Valentino Tosatti, Northwestern University, Title to be announced.

Maryna Viazovska, École Polytechnique Fédérale de Lausanne, Title to be announced.

\section{Special Sessions}

If you are volunteering to speak in a Special Session, you should send your abstract as early as possible via the abstract submission form found at www. ams . org/cgi-bin/ abstracts/abstract.p1.

Analysis and Geometry in Non-smooth Spaces (Code: SS 5A), Nageswari Shanmugalingam and Gareth Speight, University of Cincinnati.

Arithmetic Dynamics (Code: SS 1A), Jacqueline M. Anderson, Bridgewater State University, Robert Benedetto, Amherst College, and Joseph H. Silverman, Brown University.

Arrangements of Hypersurfaces (Code: SS 2A), Graham Denham, University of Western Ontario, and Alexander I. Suciu, Northeastern University.

Combinatorial Aspects of Nilpotent Orbits (Code: SS 15A), Anthony Iarrobino, Northeastern University, Leila Khatami, Union College, and Juliana Tymoczko, Smith College.

Ergodic Theory and Dynamics in Combinatorial Number Theory (Code: SS 7A), Stanley Eigen, Northeastern University, Daniel Glasscock, Ohio State University, and Vidhu Prasad, University of Massachusetts, Lowell.

Extremal Graph Theory and Quantum Walks on Graphs (Code: SS 13A), Sebastian Cioabă, University of Delaware, Mark Kempton, Harvard University, Gabor Lippner, Northeastern University, and Michael Tait, Carnegie Mellon University.

Facets of Symplectic Geometry and Topology (Code: SS 3A), Tara Holm, Cornell University, Jo Nelson, Columbia University, and Jonathan Weitsman, Northeastern University.

Geometry of Moduli Spaces (Code: SS 10A), Ana-Marie Castravet and Emanuele Macrí, Northeastern University, Benjamin Schmidt, University of Texas, and Xiaolei Zhao, Northeastern University.

Homological Commutative Algebra (Code: SS 11A), Sean Sather-Wagstaff, Clemson University, and Oana Veliche, Northeastern University.

Hopf Algebras, Tensor Categories, and Homological Algebra (Code: SS 8A), Cris Negron, Massachusetts Institute of Technology, Julia Plavnik, Texas A\&M, and Sarah Witherspoon, Texas A\&M University.

New Developments in Inverse Problems and Imaging (Code: SS 9A), Ru-Yu Lai, University of Minnesota, and Ting Zhou, Northeastern University.
Nonlinear and Stochastic Partial Differential Equations and Applications (Code: SS 19A), Nathan Glatt-Holtz and Vincent Martinez, Tulane University, and Cecilia Mondaini, Texas A\&M University.

Numerical Methods and Applications (Code: SS 16A), Vera Babenko, Ithaca College.

Polytopes and Discrete Geometry (Code: SS 6A), Gabriel Cunningham, University of Massachusetts, Boston, Mark Mixer, Wentworth Institute of Technology, and Egon Schulte, Northeastern University.

Regularity of PDEs on Rough Domains (Code: SS 14A), Murat Akman, University of Connecticut, and Max Engelstein, Massachusetts Institute of Technology.

Relations Between the History and Pedagogy of Mathematics (Code: SS 20A), Amy Ackerberg-Hastings, and David L. Roberts, Prince George's Community College.

Singularities of Spaces and Maps (Code: SS 4A), Terence Gaffney and David Massey, Northeastern University.

The Gaussian Free Field and Random Geometry (Code: SS 12A), Jian Ding, University of Chicago, and Vadim Gorin, Massachusetts Institute of Technology.

Topics in Toric Geometry (Code: SS 17A), Ivan Martino, Northeastern University, and Emanuele Ventura, Texas A\&M University.

Topology of Biopolymers (Code: SS 18A), Erica Flapan, Pomona College, and Helen Wong, Carleton College.

\section{Shanghai, People’s Republic of China}

\section{Fudan University}

June 11-14, 2018

Monday - Thursday

\section{Meeting \# 1140}

Associate secretary: Steven H. Weintraub

Announcement issue of Notices: April 2018

Program first available on AMS website: Not applicable Program issue of electronic Notices: Not applicable Issue of Abstracts: Not applicable

\section{Deadlines}

For organizers: To be announced

For abstracts: To be announced

The scientific information listed below may be dated. For the latest information, see www.ams.org/amsmtgs/ internmtgs.htm 1 .

\section{Invited Addresses}

Yu-Hong Dai, Academy of Mathematics and System Sciences, Title to be announced.

Kenneth A. Ribet, University of California, Berkeley, Title to be announced.

Richard M. Schoen, University of California, Irvine, Title to be announced. 


\section{MEETINGS \& CONFERENCES}

Sijue Wu, University of Michigan, Title to be announced. Chenyang Xu, Peking University, Title to be announced. Jiangong You, Nankai University, Title to be announced.

\section{Newark, Delaware}

\section{University of Delaware}

\section{September 29-30, 2018}

Saturday - Sunday

\section{Meeting \# 1141}

Eastern Section

Associate secretary: Steven H. Weintraub

Announcement issue of Notices: June 2018

Program first available on AMS website: August 9, 2018

Issue of Abstracts: Volume 39, Issue 3

\section{Deadlines}

For organizers: February 28, 2018

For abstracts: July 31, 2018

The scientific information listed below may be dated. For the latest information, see www. ams.org/amsmtgs/ sectional.html.

\section{Invited Addresses}

Leslie Greengard, New York University, Title to be announced.

Elisenda Grigsby, Boston College, Title to be announced. Davesh Maulik, Massachusetts Institute of Technology, Title to be announced.

\section{Fayetteville, Arkansas}

\section{University of Arkansas}

\section{October 6-7, 2018}

Saturday - Sunday

\section{Meeting \# 1142}

Southeastern Section

Associate secretary: Brian D. Boe

Announcement issue of Notices: July 2018

Program first available on AMS website: August 16, 2018 Issue of Abstracts: Volume 39, Issue 3

\section{Deadlines}

For organizers: March 6, 2018

For abstracts: August 7, 2018

The scientific information listed below may be dated. For the latest information, see www. ams.org/amsmtgs/ sectiona 7. htm 1 .

\section{Invited Addresses}

Mihalis Dafermos, Princeton University, Title to be announced.

Jonathan Hauenstein, University of Notre Dame, Title to be announced.

Kathryn Mann, University of California Berkeley, Title to be announced.

\section{Ann Arbor, Michigan}

University of Michigan, Ann Arbor

October 20-21, 2018

Saturday - Sunday

\section{Meeting \# 1143}

Central Section

Associate secretary: Georgia Benkart

Announcement issue of Notices: July 2018

Program first available on AMS website: August 30, 2018 Issue of Abstracts: Volume 39, Issue 4

\section{Deadlines}

For organizers: March 20, 2018

For abstracts: August 21, 2018

The scientific information listed below may be dated. For the latest information, see www.ams.org/amsmtgs/ sectional.htm?.

\section{Invited Addresses}

Elena Fuchs, University of Illinois Urbana-Champaign, Title to be announced.

Andrew Putman, University of Notre Dame, Title to be announced.

Charles Smart, University of Chicago, Title to be announced.

\section{Special Sessions}

If you are volunteering to speak in a Special Session, you should send your abstract as early as possible via the $a b$ stract submission form found at www. ams . org/cgi-bin/ abstracts/abstract.p1.

Geometry of Submanifolds, in Honor of Bang-Yen Chens 75th Birthday (Code: SS 1A), Alfonso Carriazo, University of Sevilla, Ivko Dimitric, Penn State Fayette, Yun Myung Oh, Andrews University, Bogdan D. Suceava, California State University, Fullerton, Joeri Van der Veken, University of Leuven, and Luc Vrancken, Universite de Valenciennes.

Interactions between Algebra, Machine Learning and Data Privacy (Code: SS 3A), Jonathan Gryak, University of Michigan, Kelsey Horan, CUNY Graduate Center, Delaram Kahrobaei, CUNY Graduate Center and New York University, Kayvan Najarian and Reza Soroushmehr, University of Michigan, and Alexander Wood, CUNY Graduate Center.

Random Matrix Theory Beyond Wigner and Wishart (Code: SS 2A), Elizabeth Meckes and Mark Meckes, Case 
Western Reserve University, and Mark Rudelson, University of Michigan.

Self-similarity and Long-range Dependence in Stochastic Processes (Code: SS 4A), Takashi Owada, Purdue University, Yi Shen, University of Waterloo, and Yizao Wang, University of Cincinnati.

Structured Homotopy Theory (Code: SS 5A), Thomas Fiore, University of Michigan, Dearborn, Po Hu, Wayne State University, Dan Isaksen, Wayne State University, and Igor Kriz, University of Michigan.

\section{San Francisco, California}

\section{San Francisco State University}

October 27-28, 2018

Saturday - Sunday

\section{Meeting \# 1144}

Western Section

Associate secretary: Michel L. Lapidus

Announcement issue of Notices: July 2018

Program first available on AMS website: September 6, 2018

Issue of Abstracts: Volume 39, Issue 4

\section{Deadlines}

For organizers: March 27, 2018

For abstracts: August 28, 2018

The scientific information listed below may be dated. For the latest information, see www.ams.org/amsmtgs/ sectiona1.htm 1 .

\section{Invited Addresses}

Srikanth B. Iyengar, University of Utah, Title to be announced.

Sarah Witherspoon, Texas A\&M University, Title to be announced.

Abdul-Aziz Yakubu, Howard University, Title to be announced.

\section{Special Sessions}

If you are volunteering to speak in a Special Session, you should send your abstract as early as possible via the abstract submission form found at www. ams . org/cgi-bin/ abstracts/abstract.p1.

Coupling in Probability and Related Fields (Code: SS 3A), Sayan Banerjee, University of North Carolina, Chapel Hill, and Terry Soo, University of Kansas.

Homological Aspects of Noncommutative Algebra and Geometry (Code: SS 2A), Dan Rogalski, University of California San Diego, Sarah Witherspoon, Texas A\&M University, and James Zhang, University of Washington, Seattle.

Mathematical Biology with a focus on Modeling, Analysis, and Simulation (Code: SS 1A), Jim Cushing, The University of Arizona, Saber Elaydi, Trinity University, Suzanne
Sindi, University of California, Merced, and Abdul-Aziz Yakubu, Howard University.

\section{Baltimore, Maryland}

Baltimore Convention Center, Hilton Baltimore, and Baltimore Marriott Inner Harbor Hotel

January 16-19,2019

Wednesday - Saturday

\section{Meeting \#1 145}

Joint Mathematics Meetings, including the 125th Annual Meeting of the AMS, 102nd Annual Meeting of the Mathematical Association of America (MAA), annual meetings of the Association for Women in Mathematics (AWM)and the National Association of Mathematicians (NAM), and the winter meeting of the Association of Symbolic Logic (ASL), with sessions contributed by the Society for Industrial and Applied Mathematics (SIAM).

Associate secretary: Steven H. Weintraub

Announcement issue of Notices: October 2018

Program first available on AMS website: To be announced Issue of Abstracts: To be announced

\section{Deadlines}

For organizers: April 2, 2018

For abstracts: To be announced

\section{Auburn, Alabama}

\section{Auburn University}

\section{March 15-17, 2019}

Friday - Sunday

\section{Meeting \# 1146}

Southeastern Section

Associate secretary: Brian D. Boe

Announcement issue of Notices: To be announced

Program first available on AMS website: To be announced Issue of Abstracts: To be announced

\section{Deadlines}

For organizers: To be announced

For abstracts: To be announced

\section{Honolulu, Hawaii}

\section{University of Hawaii at Manoa}

\section{March 22-24, 2019}

Friday - Sunday 


\section{MEETINGS \& CONFERENCES}

\section{Meeting \# 1147}

Central Section

Associate secretaries: Georgia Benkart and Michel L. Lapidus

Announcement issue of Notices: To be announced

Program first available on AMS website: To be announced Issue of Abstracts: To be announced

\section{Deadlines}

For organizers: May 15, 2018

For abstracts: January 22, 2019

The scientific information listed below may be dated. For the latest information, see www.ams.org/amsmtgs/ sectional.htm 1 .

\section{Invited Addresses}

Barry Mazur, Harvard University, Title to be announced (Einstein Public Lecture in Mathematics).

Aaron Naber, Northwestern University, Title to be announced.

Deanna Needell, University of California, Los Angeles, Title to be announced.

Katherine Stange, University of Colorado, Boulder, Title to be announced.

Andrew Suk, University of Illinois at Chicago, Title to be announced.

\section{Quy Nhon City, Vietnam}

\section{Quy Nhon University}

\section{June 10-13, 2019}

Monday - Thursday

Associate secretary: Brian D. Boe

Announcement issue of Notices: To be announced

Program first available on AMS website: To be announced Issue of Abstracts: To be announced

\section{Deadlines}

For organizers: To be announced

For abstracts: To be announced

\section{Binghamton, New York}

\section{Binghamton University}

\section{October 12-13, 2019}

Saturday - Sunday

Eastern Section

Associate secretary: Steven H. Weintraub

Announcement issue of Notices: To be announced

Program first available on AMS website: To be announced
Issue of Abstracts: To be announced

\section{Deadlines}

For organizers: March 12, 2019

For abstracts: To be announced

\section{Denver, Colorado}

\section{Colorado Convention Center}

January 15-18, 2020

Wednesday - Saturday

Joint Mathematics Meetings, including the 126th Annual Meeting of the AMS, 103rd Annual Meeting of the Mathematical Association of America (MAA), annual meetings of the Association for Women in Mathematics (AWM) and the National Association of Mathematicians (NAM), and the winter meeting of the Association of Symbolic Logic (ASL), with sessions contributed by the Society for Industrial and Applied Mathematics (SIAM)

Associate secretary: Michel L. Lapidus

Announcement issue of Notices: October 2019

Program first available on AMS website: November 1, 2019 Issue of Abstracts: To be announced

\section{Deadlines}

For organizers: April 1, 2019

For abstracts: To be announced

\section{Washington, District of Columbia}

\section{Walter E. Washington Convention Center}

\section{January 6-9, 2021}

Wednesday - Saturday

Joint Mathematics Meetings, including the 127th Annual Meeting of the AMS, 104th Annual Meeting of the Mathematical Association of America (MAA), annual meetings of the Association for Women in Mathematics (AWM) and the National Association of Mathematicians (NAM), and the winter meeting of the Association of Symbolic Logic (ASL), with sessions contributed by the Society for Industrial and Applied Mathematics (SIAM).

Associate secretary: Brian D. Boe

Announcement issue of Notices: October 2020

Program first available on AMS website: November 1, 2020 Issue of Abstracts: To be announced

\section{Deadlines}

For organizers: April 1, 2020

For abstracts: To be announced 
Name

(please print your name as you would like it to appear on your badge)

Mailing Address

Telephone

In case you have an emergency at the meeting: Day \#:___ Evening \#:

Email Address

Additional email address for receipt

Acknowledgment of this registration and any hotel reservations will be sent to the email address(es) given here. Check this box to receive a copy in U.S. Mail:

Affiliation for badge Nonmathematician guest badge name:

(company/university) (Note fee of US\$21)

In an effort to make the JMM more environmentally friendly as well as save on printing expenses to the meeting, the JMM program books will now only be distributed to participants who ask for them. More up-to-date program and meeting information will be available on the JMM website and mobile app.

Do you want to receive a copy of the program book? Yes $\square$ No $\square$

I DO NOT want my badge and program (if printed program is requested) to be mailed to me on $12 / 8 / 17$. Materials will be mailed to the address listed above unless you check this box. Materials will not be mailed for registrations completed after November 22 , or to individual commercial or artist exhibitors. Exhibiting companies may opt to have booth staff badges mailed to a company contact.

\section{Registration Fees}

Membership please $\checkmark$ all that apply. First row is eligible to register as a member. For undergraduate students, membership in PME and KME also applies.

$\square$ AMS \& MAA $\square$ AMS but not MAA $\square$ MAA but not AMS $\square$ ASL $\square$ CMS $\square$ SIAM Undergraduate Students Only: $\quad \square \mathrm{PME} \quad \square \mathrm{KME}$ Other Societies: $\square$ AWM $\square$ NAM $\square$ YMN $\square$ AMATYC

\begin{tabular}{|c|c|c|c|c|}
\hline \multicolumn{2}{|c|}{ J oint Meetings } & oy Dec 20 & at mtg & \multirow[t]{2}{*}{ Subtotal } \\
\hline$\square$ & Member AMS, MAA, ASL, CMS, or SIAM & US\$ 329 & US\$ 433 & \\
\hline$\square$ & Nonmember & US\$ 522 & US\$ 666 & \\
\hline$\square$ & Graduate Student Member (AMS, MAA & & & \\
\hline & ASL, CMS, or SIAM) & US\$ 74 & US\$ 86 & \\
\hline$\square$ & Graduate Student (Nonmember) & US\$118 & US\$ 130 & \\
\hline$\square$ & Undergraduate Student (Member AMS, & & & \\
\hline & ASL, CMS, MAA, PME, KME, or SIAM) & US\$ 74 & US\$ 86 & \\
\hline$\square$ & Undergraduate Student (Nonmember) & US\$118 & US\$ 130 & \\
\hline$\square$ & High School Student & US\$ 7 & US\$ 14 & \\
\hline$\square$ & Unemployed & US\$ 74 & US\$ 86 & \\
\hline$\square$ & Temporarily Employed & US\$ 268 & US\$ 307 & \\
\hline$\square$ & Developing Countries Special Rate & US\$ 74 & US\$ 86 & \\
\hline$\square$ & Emeritus Member of AMS or MAA & US\$ 74 & US\$ 86 & \\
\hline$\square$ & High School Teacher & US\$ 74 & US\$ 86 & \\
\hline$\square$ & Librarian & US\$ 74 & US\$ 86 & \\
\hline$\square$ & Press & US\$ 0 & US\$ 0 & \\
\hline$\square$ & Exhibitor (Commercial) & US\$ 0 & US\$ & \\
\hline$\square$ & Artist Exhibitor (work in JMM Art Exhibit) & US\$ 0 & US\$ & \\
\hline$\square$ & Nonmathematician Guest & 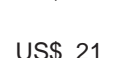 & 1 & \\
\hline
\end{tabular}
of registered mathematician $\quad$ US\$ $21 \quad$ US\$ 21

AMS Short Course: Random Growth Models (1/8-1/9)

$\square \quad$ Member of AMS

US\$ 114 US\$148

$\square \quad$ Nonmember

US\$ 175 US\$205

$\square$ Student, Unemployed, Emeritus

US\$ 62 US\$ 83

MAA Minic ourses (see listing in text)

I would like to attend: $\square$ One Minicourse $\square$ Two Minicourses

Please enroll me in MAA Minicourse(s) \#

Price: US\$ 100 for each minicourse.

\section{Graduate School Fair Table}

$\square \quad$ Graduate Program Table US\$125 US\$125

(includes table, posterboard \& electricity)

Dept. or Program to be represented (write below or email)

Receptions \& Banquets

$\square$ Graduate Student/First-Time Attendee Reception (1/10) (no charge)

$\square \quad$ NAM Banquet (1/12)

\#__Chicken \#___Fish \#___Vegan US\$ 75

\#___Kosher (Additional fees apply for Kosher Meals.) US\$ 125

Total for NAM Banquet

$\square$ AMS Dinner (1/13) Regular Price \#__US\$ 75

Student Price \#__uS\$30

(For special dietary requests, please email mmsb@ams.org)

Total for AMS Dinner

Total for Registrations and Events

\section{Payment}

Registration \& Event Total (total from column on left)

Hotel Deposit (only if paying by check)

If you send a hotel deposit check, the

deadline for this form is December 5 .

Total Amount To Be Paid

\section{Method of Payment}

$\square$ Check. Make checks payable to the AMS. For all check payments, please keep a copy of this form for your records.

$\square$ Credit Card. All major credit cards accepted. For your security, we do not accept credit card numbers by email, fax, or postal mail. If the MMSB receives your registration form by any of these methods, it will contact you at the phone number provided on this form.

Signature:

$\square$ Purchase Order \# (please enclose copy)

\section{Other Information}

Mathematical Reviews field of interest \#

$\square \quad$ I am willing to serve as a judge for the MAA Undergraduate Student Poster Session

$\square \quad$ For planning purposes for the MAA Two-year College Reception, please check if you are a faculty member at a two-year college.

$\square \quad$ I am a mathematics department chair.

$\square \quad$ Please do not include my name and postal address on any promotional mailing lists. (The JMM does not share email addresses.)

$\square \quad$ Please do not include my name on any list of JMM participants other than the scientific program if $\mathrm{I}$ am, in fact, making a presentation that is part of the meeting.

$\square$ Please $\checkmark$ this box if you have a disability requiring special services.

Registration for the Joint Meetings is not required for the short course but it is required for the minicourses and the Employment Center. To register for the Employment Center, go to http://www.ams.org/profession/employment-services/employment-center. For questions, email: emp-info@ams.org.

\section{Registration Deadlines}

To be eligible for the complimentary hotel room lottery: In time to receive badges/programs in the mail:

Hotel reservations with check deposit:

Hotel reservations, changes/cancellations

through the JMM website:

Advance registration for the Joint Meetings, short

course, minicourses, and dinner tickets:

Cancel in time to receive $50 \%$ refund on advance

registration, banquets, minicourses, and short course

*no refunds issued after this date.

Oct. 31, 2017

Nov. 22, 2017

Dec. 5, 2017

Dec. 6, 2017

Dec. 20, 2017

an. 4,2018

\section{Mailing Address/Contact:}

Mathematics Meetings Service Bureau (MMSB)

P. O. Box 6887

Providence, RI 02940-6887 Fax: 401-455-4004; Email: mmsb@ams.org

Telephone: $401-455-4144$ or 1-800-321-4267 x4144 or x4137 


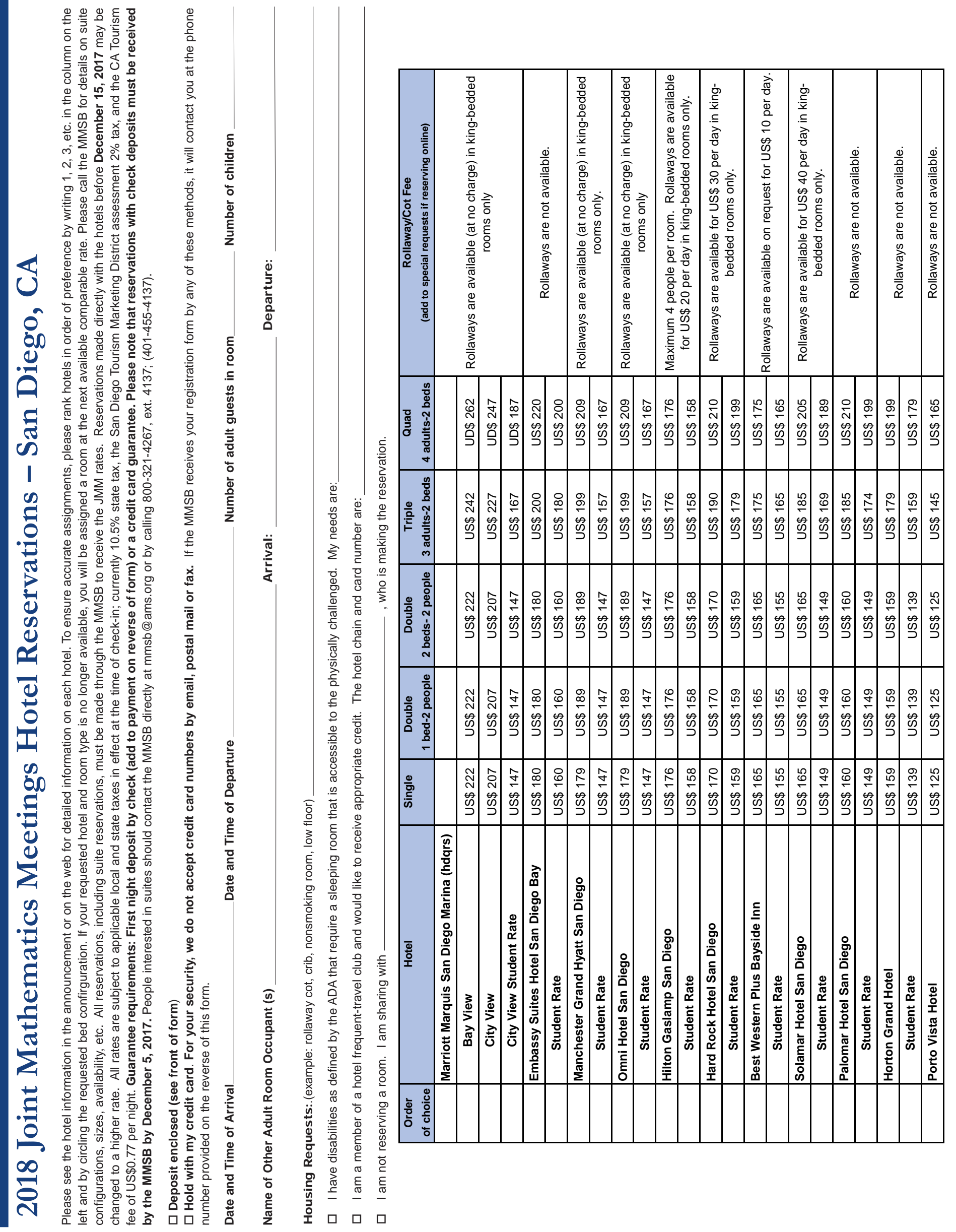




\section{IN THE NEXT ISSUE OF NOTICES}

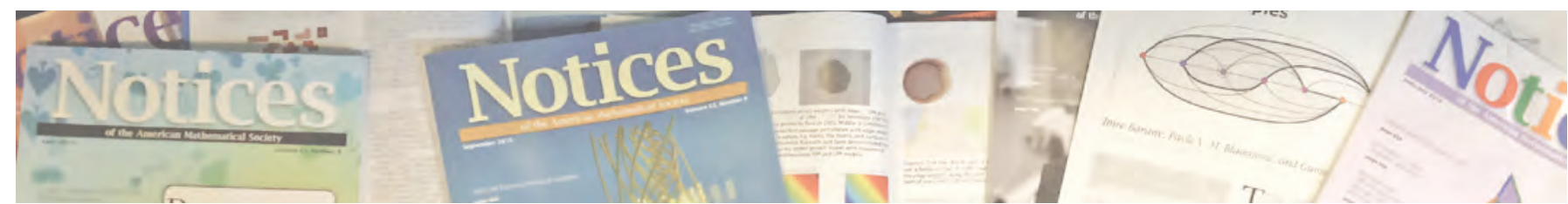

DECEMBER $2017 \ldots$

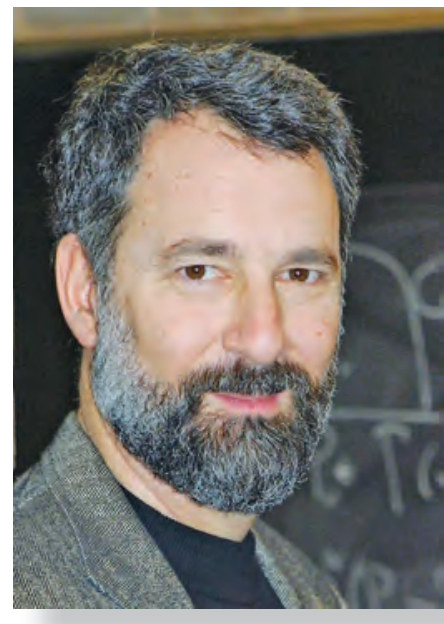

Ad Honorem Charles Fefferman

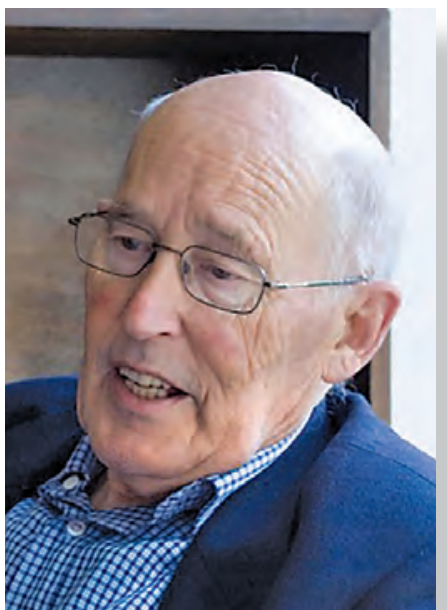

Marcel Berger Remembered

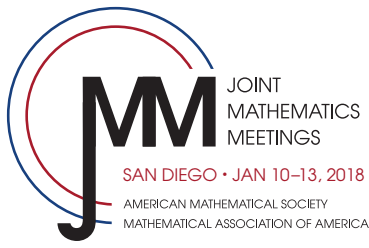

Register by November 22 to receive your badge, program, and tickets by mail before the meeting.

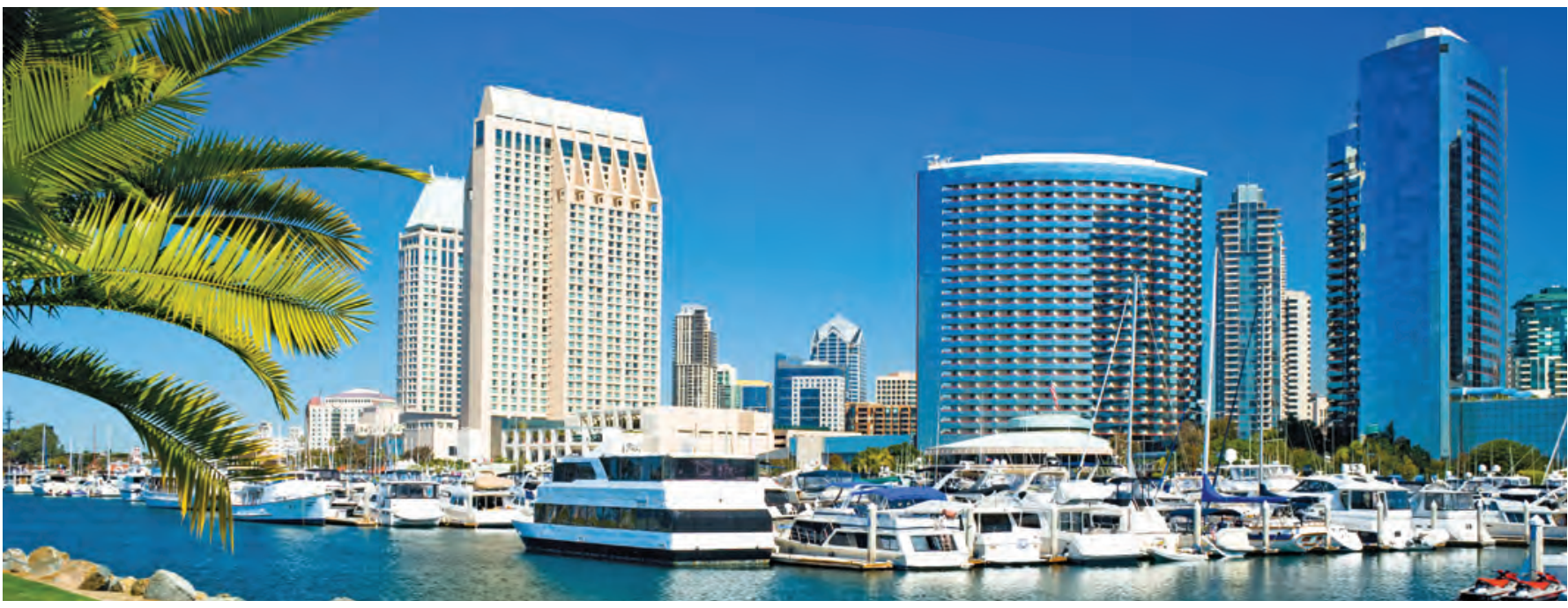

jointmathematicsmeetings.org 
American Mathematical Society

Distribution Center

35 Monticello Place,

Pawtucket, RI 02861 USA

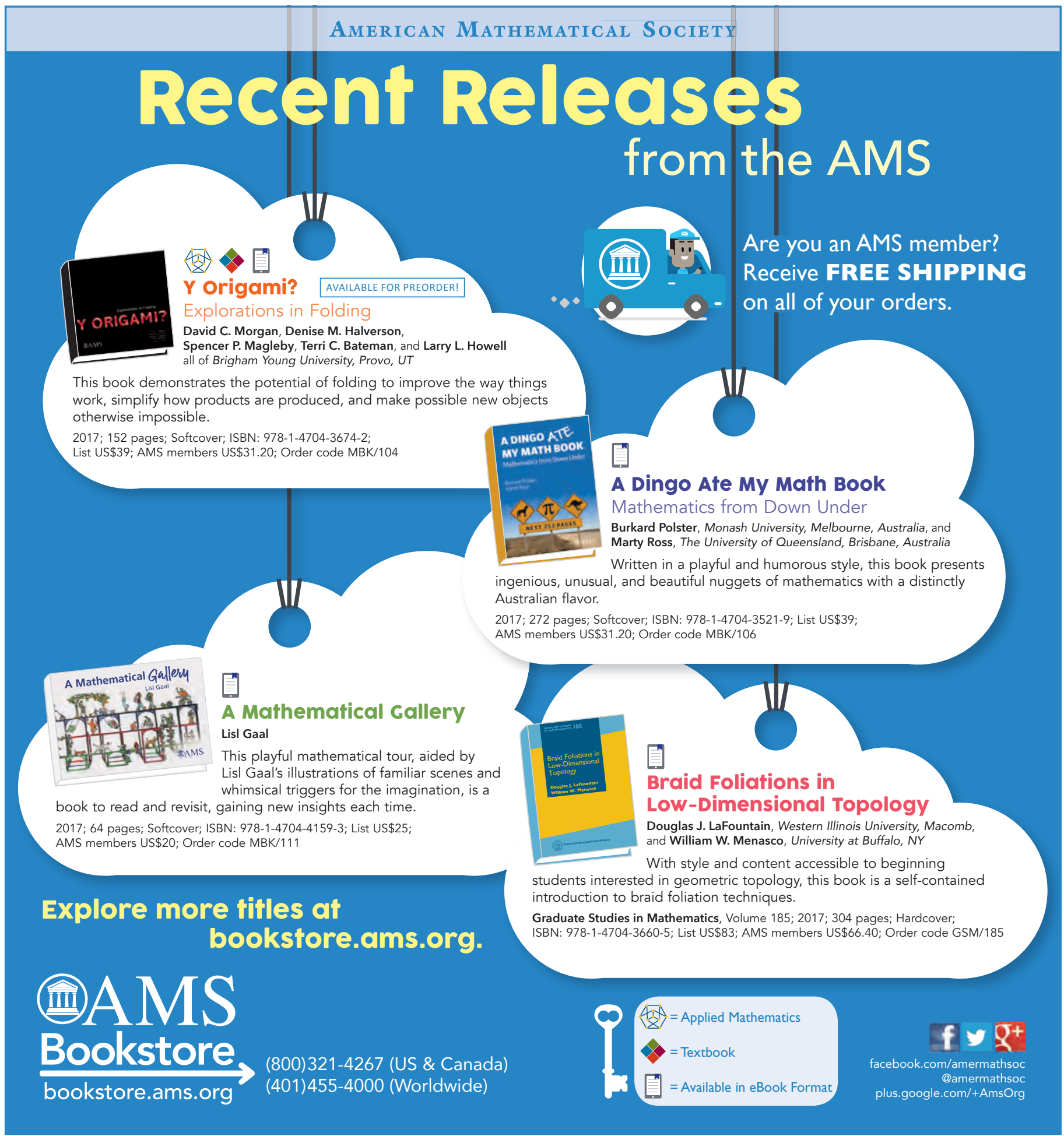

